\title{
S2k-Leitlinie zur Diagnostik und Therapie von Patienten mit Asthma
}

herausgegeben von der Deutschen Gesellschaft für Pneumologie und Beatmungsmedizin e.V. und der Deutschen Atemwegsliga e.V., unter Beteiligung der Gesellschaft für Pädiatrische Pneumologie e.V. und der Österreichischen Gesellschaft für Pneumologie

\section{Guideline for the Diagnosis and Treatment of Asthma}

\author{
Guideline of the German Respiratory Society and the German Atemwegsliga \\ in Cooperation with the Paediatric Respiratory Society and the Austrian Society \\ of Pneumology
}

Unter Mitwirkung der folgenden Wissenschaftlichen Fachgesellschaften:

Deutsche Gesellschaft für Arbeitsmedizin und Umweltmedizin e. V., Deutsche Gesellschaft für

Rehabilitationswissenschaften e.V. und Deutsche Gesellschaft für Gynäkologie und Geburtshilfe e.V.

\section{Autoren}

R. Buhl ${ }^{1}$, R. Bals², X. Baur ${ }^{3}$, D. Berdel ${ }^{4}$, C.-P. Criée ${ }^{5}$, M. Gappa ${ }^{6}$, A. Gillissen ${ }^{7}$, T. Greulich ${ }^{8}$, P. Haidl ${ }^{9}$, E. Hamelmann ${ }^{10}$, P. Kardos ${ }^{11}$, K. Kenn ${ }^{12}$, L. Klimek ${ }^{13}$, S. Korn ${ }^{1}$, M. Lommatzsch ${ }^{14}$, H. Magnussen ${ }^{15}$, T. Nicolai ${ }^{16}$, D. Nowak ${ }^{17}$, O. Pfaar ${ }^{13,18}$, K. F. Rabe ${ }^{19}$, J. Riedler ${ }^{20}$, T. Ritz ${ }^{21}$, K. Schultz ${ }^{22}$, A. Schuster ${ }^{23}$, T. Spindler ${ }^{24}$, C. Taube ${ }^{25}$, K. Taube ${ }^{26}$, C. Vogelmeier ${ }^{8}$, A. von Leupoldt ${ }^{27}$, F. Wantke ${ }^{28}$, S. Weise ${ }^{29}$, J. Wildhaber ${ }^{30}$, H. Worth ${ }^{31}$, A. Zacharasiewicz ${ }^{32}$

Institute

1 Universitätsmedizin der Johannes GutenbergUniversität Mainz, Schwerpunkt Pneumologie, III. Medizinische Klinik, Mainz

2 Universitätsklinikum des Saarlandes, Klinik für Innere Medizin V, Homburg/Saar

3 European Society for Environmental and Occupational Medicine, EOM, Berlin

4 im Ruhestand, Hamminkeln

5 Evangelisches Krankenhaus Göttingen Weende, Abteilung für Pneumologie, Bovenden-Lenglern

6 Marien-Hospital gGmbH, Klinik für Kinder- und Jugendmedizin, Wesel

7 Klinikum Am Steinenberg, Ermstalklinik, Medizinische Klinik III/Innere Medizin und Pneumologie, ReutlingenBad Urach

8 Universitätsklinikum Gießen und Marburg, Klinik für Innere Medizin mit Schwerpunkt Pneumologie, Marburg

9 Fachkrankenhaus Kloster Grafschaft GmbH, Abteilung Pneumologie II, Schmallenberg

10 Evangelisches Klinikum Bethel, Klinik für Kinder- und Jugendmedizin, Bielefeld

11 Lungenpraxis an der Klinik Maingau vom Roten Kreuz, Frankfurt am Main
12 Schön Klinik Berchtesgadener Land, Fachzentrum für Pneumologie, Schönau am Königssee, Philipps Universität Marburg, Standort Schönau

13 Zentrum für Rhinologie und Allergologie, Wiesbaden

14 Universitätsmedizin Rostock, Abteilung Pneumologie, Rostock

15 Pneumologisches Forschungsinstitut an der LungenClinic Grosshansdorf GmbH, Großhansdorf

16 Klinikum der Universität München, Kinderklinik und Kinderpoliklinik, LMU München

17 Klinikum der Universität München, Institut und Poliklinik für Arbeits-, Sozial- und Umweltmedizin, LMU München

18 HNO-Universitätsklinik Mannheim, Medizinische Fakultät Mannheim, Universität Heidelberg, Mannheim

19 LungenClinic Grosshansdorf $\mathrm{GmbH}$, Abteilung für Pneumologie, Großhansdorf

20 Kardinal Schwarzenberg Klinikum, Kinder- und Jugendmedizin, Schwarzach im Pongau, Österreich

21 Southern Methodist University, Department of Psychology, Dallas, USA

22 Klinik Bad Reichenhall, Fachbereich Pneumologie, Bad Reichenhall

23 Universitätsklinikum Düsseldorf, Zentrum für Kinderund Jugendmedizin, Düsseldorf 
24 Waldburg-Zeil Kliniken, Fachkliniken Wangen, Klinik für Pädiatrische Pneumologie und Allergologie, Rehabilitationsklinik für Kinder und Jugendliche, Wangen

25 Universitätsmedizin Essen, Ruhrlandklinik, Klinik für Pneumologie, Essen

26 Atem-Reha GmbH, Hamburg

27 University of Leuven, Health Psychology, Leuven, Belgien

28 Floridsdorfer Allergiezentrum, Wien, Österreich

29 Atem- und Physiotherapie Solln, München

30 HFR Freiburg, Kantonsspital, Klinik für Pädiatrie, Freiburg, Schweiz

31 Facharztzentrum Fürth, Fürth

32 Wilhelminenspital, Lehrkrankenhaus der Medizinischen Universität Wien, Abteilung für Kinderund Jugendheilkunde, Wien, Österreich

Bibliografie

DOI https://doi.org/10.1055/s-0043-119504 |

Pneumologie 2017; 71: 849-919

(c) Georg Thieme Verlag KG Stuttgart · New York ISSN 0934-8387

\section{Korrespondenzadresse}

Prof. Dr. med. Roland Buhl, III. Medizinische Klinik, Schwerpunkt Pneumologie, Universitätsmedizin der Johannes Gutenberg-Universität Mainz, Langenbeckstraße 1, 55131 Mainz roland.buhl@unimedizin-mainz.de

\section{ZUSAMMENFASSUNG}

Die vorliegende Leitlinie ist eine Neufassung und Aktualisierung der Leitlinie zur Diagnostik und Therapie von Patienten mit Asthma, welche die bisher für den deutschen Sprachraum gültige Version aus dem Jahr 2006 ablöst. Die Fülle an neuen Erkenntnissen zur Pathophysiologie und zu den Phänotypen von Asthma und das erweiterte Spektrum an diagnostischen und therapeutischen Möglichkeiten bei dieser Erkrankung machte eine Neufassung und Aktualisierung notwendig. Es werden sowohl für Kinder und Jugendliche als auch für Erwachsene mit Asthma die aktuellen, Evidenz-basierten diagnostischen und therapeutischen Empfehlungen dargelegt.

\section{ABSTRACT}

The present guideline is a new version and an update of the guideline for the diagnosis and treatment of asthma, which replaces the previous version for german speaking countries from the year 2006. The wealth of new data on the pathophysiology and the phenotypes of asthma, and the expanded spectrum of diagnostic and therapeutic options necessitated a new version and an update. This guideline presents the current, evidence-based recommendations for the diagnosis and treatment of asthma, for children and adolescents as well as for adults with asthma. 


\begin{tabular}{|c|c|c|}
\hline \multicolumn{3}{|c|}{ Inhaltsverzeichnis } \\
\hline 1 & Einleitung & 852 \\
\hline 2 & Struktur des Leitlinienprozesses & 852 \\
\hline 3 & Definition von Asthma & 853 \\
\hline 3.1 & Charakterisierung der Erkrankung & 853 \\
\hline 3.1 .1 & Pathophysiologie & 853 \\
\hline 3.2 & $\begin{array}{l}\text { Faktoren, die die Entwicklung und den Schweregrad } \\
\text { eines Asthmas beeinflussen }\end{array}$ & 853 \\
\hline 3.2 .1 & Endogene Faktoren & 854 \\
\hline 3.2 .2 & Exogene Faktoren & 854 \\
\hline 3.3 & Asthmaformen & 855 \\
\hline 3.3 .1 & $\begin{array}{l}\text { Allergisches (extrinsisches) versus nicht allergisches } \\
\text { (intrinsisches) Asthma }\end{array}$ & 855 \\
\hline 3.3 .2 & „Type-2-High“- versus „Type-2-Low“-Asthma & 856 \\
\hline 3.3 .3 & Eosinophiles versus nicht eosinophiles Asthma & 856 \\
\hline 3.3.4 & $\begin{array}{l}\text { Cough-variant Asthma („Husten als Asthma- } \\
\text { Äquivalent“) }\end{array}$ & 857 \\
\hline 3.3 .5 & Sonstige Asthmaformen & 857 \\
\hline 3.4 & Schweres Asthma & 858 \\
\hline 3.5 & Epidemiologie und ökonomische Bedeutung & 858 \\
\hline 3.6 & Verlauf der Erkrankung und Mortalität & 858 \\
\hline 4 & Diagnose des Asthmas & 859 \\
\hline 4.1 & Anamnese & 859 \\
\hline 4.1 .1 & Erwachsene & 859 \\
\hline 4.1 .2 & Kinder & 860 \\
\hline 4.2 & Klinische Untersuchungsbefunde & 860 \\
\hline 4.3 & Lungenfunktionsdiagnostik & 860 \\
\hline 4.3 .1 & Spirometrie & 860 \\
\hline 4.3 .2 & Ganzkörperplethysmografie & 862 \\
\hline 4.3 .3 & Diffusionskapazität für Kohlenmonoxid & 862 \\
\hline 4.3 .4 & Arterielle Blutgasanalyse & 862 \\
\hline 4.3 .5 & $\begin{array}{l}\text { Exspiratorischer Spitzenfluss (Peak-exspiratory-flow, } \\
\text { PEF) }\end{array}$ & 862 \\
\hline 4.4 & Nicht invasive Diagnostik der Atemwegsentzündung & 862 \\
\hline 4.4 .1 & Atemkondensat & 862 \\
\hline 4.4 .2 & Spontanes oder induziertes Sputum & 862 \\
\hline 4.4 .3 & Stickstoffmonoxid (FeNO) in der Ausatemluft & 862 \\
\hline 4.5 & Röntgendiagnostik & 863 \\
\hline 4.6 & Labordiagnostik & 863 \\
\hline 4.7 & Mikrobiologische Sputumdiagnostik & 863 \\
\hline 4.8 & Allergologische Stufendiagnostik & 863 \\
\hline 4.8 .1 & Allergieanamnese & 864 \\
\hline 4.8 .2 & Allergie-Hauttestungen & 864 \\
\hline 4.8 .3 & $\begin{array}{l}\text { Bestimmung des spezifischen IgE und Gesamt-IgE } \\
\text { im Serum }\end{array}$ & 864 \\
\hline 4.8 .4 & Spezifische nasale und bronchiale Provokation & 864 \\
\hline 4.8 .5 & Diagnostische Expositionskarenz & 864 \\
\hline 4.9 & $\begin{array}{l}\text { Allgemeine Anmerkungen zur Diagnostik und } \\
\text { Differenzialdiagnostik des Asthmas }\end{array}$ & 864 \\
\hline
\end{tabular}

\begin{tabular}{|c|c|c|}
\hline 5 & Medikamentöse Therapie des Asthmas & 867 \\
\hline 5.1 & $\begin{array}{l}\text { Medikamentöse Therapie erwachsener Asthma- } \\
\text { patienten }\end{array}$ & 870 \\
\hline 5.1 .1 & Anstrengungsinduziertes Asthma & 870 \\
\hline 5.1 .2 & $\begin{array}{l}\text { Therapiestufe 1: Kurzwirksames Bedarfs- } \\
\text { therapeutikum }\end{array}$ & 872 \\
\hline 5.1 .3 & $\begin{array}{l}\text { Therapiestufe 2: Langzeittherapeutikum plus } \\
\text { kurzwirksames Bedarfstherapeutikum }\end{array}$ & 872 \\
\hline 5.1 .4 & $\begin{array}{l}\text { Therapiestufe 3: } 2 \text { Langzeittherapeutika plus } \\
\text { kurzwirksames Bedarfstherapeutikum }\end{array}$ & 873 \\
\hline 5.1 .5 & $\begin{array}{l}\text { Therapiestufe 4: } 2 \text { oder mehr Langzeittherapeutika } \\
\text { plus kurzwirksames Bedarfstherapeutikum }\end{array}$ & 874 \\
\hline 5.1 .6 & Therapiestufe 5: Additive Therapie & 875 \\
\hline 5.2 & (Allergen-)spezifische Immuntherapie (SIT) & 877 \\
\hline 5.3 & Inhalationssysteme-Verordnung, Technik, Training & 878 \\
\hline 6 & Akuttherapie bei Asthma bronchiale & 879 \\
\hline 6.1 & $\begin{array}{l}\text { Leichte und mittelschwere Asthmaexazerbation } \\
\text { beim Erwachsenen }\end{array}$ & 880 \\
\hline 6.2 & Schwere Asthmaexazerbation beim Erwachsenen & 880 \\
\hline 6.3 & $\begin{array}{l}\text { Lebensbedrohlicher Anfall (Krankenhauseinweisung } \\
\text { mit Notarztbegleitung) }\end{array}$ & 880 \\
\hline 6.4 & $\begin{array}{l}\text { Therapiemaßnahmen während des Transportes zum } \\
\text { Krankenhaus und im Krankenhaus bei unzureichen- } \\
\text { dem Ansprechen auf die Initialtherapie }\end{array}$ & 880 \\
\hline 6.5 & $\begin{array}{l}\text { Zur Behandlung des Asthmaanfalls sollten nicht } \\
\text { eingesetzt bzw. möglichst vermieden werden }\end{array}$ & 880 \\
\hline 6.6 & Erhöhtes Mortalitätsrisiko im Asthmaanfall & 881 \\
\hline 6.7 & $\begin{array}{l}\text { Indikation zur intensivmedizinischen Überwachung } \\
\text { oder Behandlung }\end{array}$ & 881 \\
\hline 6.8 & Kriterien zur Entlassung aus stationärer Behandlung & 881 \\
\hline 6.9 & Nachsorge & 881 \\
\hline 6.10 & Therapie des schweren Asthmaanfalles beim Kind & 881 \\
\hline 6.11 & Asthma und Beatmung & 882 \\
\hline 6.11 .1 & Invasive Beatmung & 882 \\
\hline 6.11 .2 & Nicht invasive Beatmung (NIV) & 882 \\
\hline 7 & Nicht medikamentöse Therapie & 883 \\
\hline 7.1 & Physiotherapie und Atemphysiotherapie & 883 \\
\hline 7.2 & Alternative Methoden & 883 \\
\hline 7.3 & Rehabilitation & 884 \\
\hline 7.4 & Patientenschulung & 887 \\
\hline 7.5 & Körperliches Training & 888 \\
\hline 7.6 & Tabakentwöhnung & 888 \\
\hline 7.7 & $\begin{array}{l}\text { Die Kontrolle des Körpergewichts bei adipösen } \\
\text { Patienten }\end{array}$ & 888 \\
\hline 7.8 & Bronchiale Thermoplastie & 888 \\
\hline 8 & Asthma und Komorbiditäten & 888 \\
\hline 8.1 & Asthma und Reflux & 888 \\
\hline 8.2 & Asthma und obstruktives Schlafapnoesyndrom & 889 \\
\hline 8.3 & Asthma und endokrinologische Störungen & 889 \\
\hline 8.4 & Asthma und Adipositas & 889 \\
\hline 8.5 & Asthma und COPD & 889 \\
\hline
\end{tabular}




\begin{tabular}{|c|c|c|}
\hline 8.6 & Asthma und Rhinitis & 890 \\
\hline 8.7 & Asthma und Psyche & 891 \\
\hline 9 & Prävention & 891 \\
\hline 9.1 & Ernährung & 892 \\
\hline 9.2 & Kontrolle des Körpergewichts & 893 \\
\hline 9.3 & Innenraumbelastung & 893 \\
\hline 9.4 & Tabakrauchexposition & 893 \\
\hline 9.5 & Inhalationsallergene & 893 \\
\hline 9.6 & Hausstaubmilben & 893 \\
\hline 9.7 & Haustiere & 893 \\
\hline 9.8 & Schimmel und Feuchtigkeit & 894 \\
\hline 9.9 & Außenraumbelastung & 894 \\
\hline 9.10 & Weitere Empfehlungen & 894 \\
\hline 10 & Arbeitsbedingtes Asthma & 894 \\
\hline 10.1 & Arbeitsmedizinische Vorsorgen & 896 \\
\hline 10.2 & Diagnostik bei Verdacht auf Berufsasthma & 896 \\
\hline 10.2 .1 & Anamnese & 896 \\
\hline 10.2 .2 & Spezielle Arbeitsanamnese: Tätigkeitsbeschreibung & 896 \\
\hline 10.2 .3 & Weitere potenzielle Risikofaktoren & 896 \\
\hline 10.2 .4 & Symptome & 896 \\
\hline 10.2 .5 & Klinische Befunde und Lungenfunktionsmessungen & 897 \\
\hline 10.2 .6 & Allergologische Diagnostik & 898 \\
\hline 10.2 .7 & Entzündungsmonitoring & 898 \\
\hline 10.3 & $\begin{array}{l}\text { Berufskrankheitenanzeige und Berufskrankheits- } \\
\text { verfahren }\end{array}$ & 898 \\
\hline 10.3.1 & BK Nr. 4301 & 899 \\
\hline 10.3 .2 & BK Nr. 4302 & 899 \\
\hline 10.3.3 & BK Nr. 1315 & 899 \\
\hline 10.4 & Verlauf, Prognose und Therapie & 899 \\
\hline 10.5 & Prävention & 899 \\
\hline 11 & Asthma in der Schwangerschaft & 899 \\
\hline 11.1 & Beratung & 900 \\
\hline 11.2 & Kontrolluntersuchungen & 900 \\
\hline 11.3 & Pharmakotherapie & 900 \\
\hline 11.4 & Spezifische Immuntherapie & 900 \\
\hline 11.5 & Schutzimpfungen & 900 \\
\hline 11.6 & Behandlung von Atemwegsinfekten & 900 \\
\hline 11.7 & Behandlung des Asthmaanfalls & 901 \\
\hline 11.8 & Management während der Geburt & 901 \\
\hline 11.9 & Geburtseinleitung bei Asthma & 901 \\
\hline 11.10 & Stillzeit & 901 \\
\hline \multicolumn{2}{|c|}{ Literatur } & 901 \\
\hline
\end{tabular}

\section{Einleitung}

Die vorliegende Leitlinie ist ein Update der Leitlinie zur Diagnostik und Therapie von Patienten mit Asthma und löst die bisher für den deutschen Sprachraum gültige Version ab [1]. Nicht nur neue Erkenntnisse in der Diagnostik und Therapie des Asthmas einschließlich der Entwicklung neuer Medikamente und Therapieoptionen machten eine Neufassung notwendig, sondern auch die Neuorientierung und das Umdenken durch die Charakterisierung von Phänotypen des Asthmas, die neue Einteilung der Schweregrade des Asthmas und den Aufbau der Stufenpläne. Auch wenn in den letzten Jahren die Zunahme der Asthmaprävalenz in westlichen Ländern zum Stillstand gekommen zu sein scheint, hat die Prävalenz von Asthma in den vergangenen Jahrzehnten in vielen Ländern zugenommen (insbesondere bei Kindern und Jugendlichen). Asthma bleibt eine der häufigsten chronischen Erkrankungen in Deutschland und die volkswirtschaftliche Bedeutung von Asthma ist sehr groß, sodass die Aktualisierung dieser Leitlinie dringend notwendig wurde.

Die wesentliche strukturelle Änderung der Leitlinie ist die Erstellung der zahlreichen Empfehlungen und Statements, die dem Leser als zusammenfassendes Nachschlagewerk dienen sollen. Die Leitlinie umfasst die Empfehlungen für Erwachsene, Jugendliche und Kinder, um eine möglichst große Zielgruppe zu erreichen. Die übersichtlichen Darstellungen in Tabellen und Abbildungen zu den relevanten Themen wie Therapieplan und Dosierungen sollen den Überblick erleichtern. Alle Kapitel bilden den aktuellen Stand der Erkenntnisse ab, wobei in diese Leitlinie nur bewährte, evidenzbasierte und für die klinische Praxis relevante Aussagen aufgenommen wurden. Die Leitlinie wendet sich an die pneumologisch ausgerichteten Fachärzte, die pädiatrische oder erwachsene Patienten mit Asthma im niedergelassenen Bereich und im Krankenhaus betreuen.

Die Leitlinie hat in wesentlichen Punkten Gemeinsamkeiten mit der aktuellen GINA-Leitlinie [2], sie berücksichtigt jedoch die Besonderheiten des deutschsprachigen Raums. Dieser Leitlinie der wissenschaftlichen Fachgesellschaften und die sich in Bearbeitung befindende Nationale Versorgungsleitlinie Asthma ergänzen sich und stehen inhaltlich nicht in Widerspruch.

\section{Struktur des Leitlinienprozesses}

Die Leitlinie wurde nach dem System der AWMF entwickelt, um dem Nutzer Kriterien für eine rationale Diagnostik und Therapie an die Hand zu geben [3]. Für die Leitlinie wurde die Entwicklungsstufe S2k angestrebt. Bei der Erstellung der Leitlinie handelt es sich um einen zweistufigen Prozess. Die für das Asthmamanagement wichtigen Inhalte für die Empfehlungen wurden zuerst innerhalb der gesamten Leitliniengruppe gemeinsam identifiziert und intensiv diskutiert. Es wurde jeweils kapitelweise eine Literatursuche in PubMed mit den von den Autoren vorgegebenen Stichwörtern durchgeführt und die Ergebnisse den Autoren über den Scientific Guideline Manager (Institut für Lungenforschung $\mathrm{GmbH}$ ) zur Unterstützung der inhaltlichen Aktualisierung der Leitlinie zur Verfügung gestellt. Darüber hinaus wurden Literaturstellen aus der letzten Version der 
Leitlinie, andere Leitlinien sowie aktuelle Studien in Abstimmung berücksichtigt, soweit sie Einfluss auf die Inhalte der aktuellen Leitlinie haben.

Auf der Basis der Fachexpertise der ausgewählten Autoren und der vorhandenen Evidenz wurden dann von den Arbeitsgruppen die Empfehlungen bzw. Statements formuliert. Parallel wurden von den Arbeitsgruppen die Hintergrundtexte, die die Empfehlungen und Statements unterstützen, verfasst bzw. aktuallisiert. Der aus diesem Prozess hervorgegangene Entwurf des Gesamtmanuskriptes wurde auf zwei Konsensuskonferenzen unter Leitung eines unabhängigen Moderators ausführlich diskutiert und überarbeitet. Nach den Ergänzungen und Überarbeitungen der einzelnen Arbeitsgruppen nach den Konsensuskonferenzen wurde das Manuskript durch die Redaktionsgruppe bearbeitet und anschließend das Gesamtliteraturverzeichnis eingefügt. Das Manuskript mit allen Empfehlungen und Statements wurde nach dieser Bearbeitung an die Leitliniengruppe übersandt. Daraus entstandene Änderungsvorschläge wurden sortiert und eingearbeitet. Im Anschluss wurde das Manuskript erneut der Leitliniengruppe im Delphi-Verfahren vorgelegt und schließlich im Konsens verabschiedet. Weitere Informationen sind dem Leitlinienreport zu entnehmen.

\section{Definition von Asthma}

Asthma ist eine heterogene, multifaktorielle, meist chronischentzündliche Erkrankung der Atemwege, die meist durch eine bronchiale Hyperreagibilität und/oder eine variable Atemwegsobstruktion charakterisiert ist und sich klinisch durch respiratorische Symptome (Luftnot, Brustenge, Giemen, Husten) wechselnder Intensität und Häufigkeit äußern kann.

\subsection{Charakterisierung der Erkrankung}

Asthma ist charakterisiert durch:

- das Auftreten respiratorischer Symptome wie Luftnot, Brustenge, Giemen oder Husten, welche in Intensität und Häufigkeit variieren (Anamnese),

- eine reversible Bronchialobstruktion unterschiedlicher Ausprägung und/oder eine bronchiale Hyperreagibilität (Lungenfunktionsprüfung), und

- eine typischerweise chronische Entzündung der Atemwege (Entzündungsmarker), in Verbindung mit strukturellen Umbauprozessen (Pathologie).

Dies gilt unabhängig vom Asthmaschweregrad. Die unbehandelte Erkrankung führt zu rezidivierenden Episoden von Atemnot, typischen Atemnebengeräuschen sowie Engegefühl in der Brust und/oder Husten, besonders nachts und in den frühen Morgenstunden. Derartige Asthmabeschwerden sind meist entweder spontan oder nach adäquater Behandlung reversibel. Vermehrte Sputumproduktion als Zeichen der Hypersekretion kann ebenfalls ein Asthmasymptom sein. Als Asthmaexazerbationen werden Phasen einer progredienten Zunahme der Asthmasymptome und/oder Abnahme der Lungenfunktion bezeichnet, welche über das für den Patienten übliche Maß an Variabilität hinausgehen und welche einer Änderung bzw. Intensivierung der Therapie über mehrere Tage bedürfen. Der Begriff
Exazerbation entspricht dem Begriff „akutes Asthma“ und schließt den Begriff „Asthmaanfall“ ein. Die Beschwerden im Rahmen von Exazerbationen können nur gering ausgeprägt oder auch schwergradig sein und ohne adäquate Behandlung bis zum Tode führen. Gehäufte Exazerbationen sind nicht automatisch mit einem schweren Asthma gleichzusetzen, da sie auch Ausdruck einer fehlenden oder unzureichenden Basistherapie, einer mangelnden Adhärenz oder persistierender Asthmatrigger sein können.

Die Limitierung des Atemflusses wird wesentlich durch vier Mechanismen verursacht:

- neuromuskulär-vermittelte Bronchokonstriktion

- Ödem der Atemwegswände

- Verdickung der Atemwegswände durch Atemwegsumbau („Remodeling“)

- Verlegung der Atemwege durch ein hochvisköses Sekret

\subsubsection{Pathophysiologie}

Asthma ist eine heterogene Erkrankung, sowohl bezüglich der Pathologie und klinischen Phänotypen als auch bezüglich des therapeutischen Ansprechens [4]. Das breite pathophysiologische Spektrum, welches zu den klinischen Symptomen eines Asthmas führen kann, beinhaltet folgende Mechanismen [5] $(\triangleright$ Tab. 1$)$ :

- epitheliale und subepitheliale Veränderungen

- immunologische Veränderungen

- neuromuskuläre Veränderungen

- vaskuläre Veränderungen

Durch Freisetzung von Zytokinen, Chemokinen und Wachstumsfaktoren sind diese zellulären Systeme miteinander vernetzt und beeinflussen sich gegenseitig. Patienten mit Asthma können in einem oder mehreren der oben genannten Systeme schwerpunktmäßig Veränderungen aufweisen und können sich daher voneinander klinisch, diagnostisch und therapeutisch unterscheiden. Trotz dieser Unterschiede haben die verschiedenen Asthmaformen das Merkmal der Atemwegshyperreagibilität meist gemeinsam. Neuromuskuläre Hyperreagibilität [6] und geometrische Veränderungen infolge der verdickten Atemwegswand [7] werden als wichtigste Effektor-Mechanismen der Atemwegshyperreagibilität diskutiert. Die zur Messung der Atemwegsreagibilität eingesetzten Substanzen (wie Methacholin, Histamin oder Kaltluft) detektieren verschiedene Aspekte der zellulären Veränderungen in den Atemwegen und können daher in Einzelfällen diskrepante Befunde ergeben. In der klinischen Manifestation ist die Hyperreagibilität jedoch relativ uniform und weitgehend unabhängig vom Asthmasubtyp.

\subsection{Faktoren, die die Entwicklung und den Schweregrad eines Asthmas beeinflussen}

Einflussfaktoren, welche die Entwicklung von Asthma oder den Schweregrad von Asthma oder beides beeinflussen, können in endogene und exogene Faktoren unterschieden werden [29]. 
> Tab. 1 Zelluläre und funktionelle Veränderungen bei Asthma.

\section{Zelluläre und funktionelle Veränderungen bei Asthma}

epitheliale und subepitheliale Veränderungen

immunologische Veränderungen
- respiratorisches Epithel: Freisetzung proinflammatorischer Mediatoren und diverser Wachstumsfaktoren infolge einer Stimulation durch Allergene und/ oder virale bzw. bakterielle Erreger; veränderte Menge und Zusammensetzung des freigesetzten Mucus $[8,9]$

- Fibrobasten/Myofibroblasten: veränderte Produktion von Bindegewebskomponenten wie Kollagenen oder Proteoglykanen, charakteristische Veränderungen der subepithelialen Matrix [10]

- dendritische Zellen: Auslöser der Sensibilisierung gegenüber Allergenen, Polarisierung von Antigen-spezifischen T-Zellen in pro-inflammatorische Subtypen (Th2, Th9, Th17, u. a.) [11,12]

- Antigen-spezifische T-Lymphozyten: erhöhte Anzahl in den Atemwegen, Freisetzung von Zytokinen wie IL-4, IL-5, IL-9 und IL-13, welche die Vermehrung und Aktivierung von Eosinophilen und die IgE-Produktion durch Plasmazellen steuern [13]

" „innate lymphoid cells“ vom Typ 2 („,ILC2“): Antigen-unspezifische lymphoide Zellen des angeborenen Immunsystems, welche in erhöhter Anzahl in den Atemwegen bestimmter Asthmaformen auftreten und Zytokine wie IL-5 und IL-13 produzieren [14, 15]

- Mastzellen: erhöhte Anzahl in den Atemwegen, Aktivierung durch IgE-Kreuzvernetzung, Freisetzung proinflammatorischer Mediatoren [16]

- eosinophile Granulozyten: erhöhte Anzahl in den Atemwegen, Korrelation mit dem Schweregrad der Erkrankung, Freisetzung von diversen Mediatoren und Wachstumsfaktoren [17, 18]

- neutrophile Granulozyten: bei schweren Asthmaformen, Exazerbationen oder Zigarettenrauchern mit Asthma vermehrt, pathophysiologische Rolle bislang ungewiss [19]

- Thrombozyten: veränderte Aktivität bei Asthma, erhöhte Freisetzung des Neurotrophins Brain-Derived Neurotrophic Factor (BDNF), welches bronchiale Hyperreagibilität auslösen kann [20 - 23]

neuromuskuläre Veränderungen

- glatte Muskulatur: durch Hypertrophie und Hyperplasie vermehrt, Vermittlung der Konstriktion der Atemwege, Freisetzung von proinflammatorischen Mediatoren und von Wachstumsfaktoren [6]

- sensorische Nerven: erhöhte Sensibilität, erhöhte Reflexaktivität, veränderte Produktion und Freisetzung von Neuropeptiden, proinflammatorische Effekte, Modulation durch Neurotrophine [24]

- parasympathische Nerven: erhöhte Neurotransmitterfreisetzung, veränderte Rezeptor-Expression, M2-RezeptorDysfunktion, Modulation durch Neurotrophine [22, 25, 26]

vaskuläre Veränderungen veränderte Extravasation von Immunzellen und Mediatoren [27, 28]
- Blutgefäße: erhöhte Anzahl der Blutgefäße in der Mucosa und Submucosa, veränderte Gefäß-Reaktivität,

\subsubsection{Endogene Faktoren}

- Genetische Prädisposition

Asthma hat eine starke erbliche Komponente. Diese ist jedoch sehr komplex und nicht auf einige wenige Gene zu reduzieren und nicht für alle Formen von Asthma homogen. Die multiplen genetischen Einflüsse auf die Entwicklung von Asthma, den Asthmaphänotyp und das Ansprechen auf Medikamente sind weiterhin Gegenstand der Forschung [30].

- Körpergewicht

Adipositas ist ein Risikofaktor für die Entwicklung, für einen ungünstigen Verlauf, einen erhöhten Schweregrad und ein mangelndes therapeutisches Ansprechen der Erkrankung. Hierbei scheinen sowohl mechanische Faktoren als auch Mediatoren wie Adipokine eine Rolle zu spielen [31]. Adipöse Patienten mit Asthma zeigen einen akzelerierten Abfall der $\mathrm{FEV}_{1}$ [32] und eine geringere Ansprechrate auf Glucocorticosteroide [33]. Bei Adipositas kann es auch zu falsch-positiven Ergebnissen der Hyperreagibilitätsmessung und somit zu Asthmafehldiagnosen kommen [34].

- Geschlecht

Das Geschlecht hat einen Einfluss auf die Entwicklung der Erkrankung, dieser Einfluss ist jedoch komplex und scheint sich im Laufe des Lebens zu wandeln. So ist im Kindesalter die Asthmaprävalenz beim männlichen Geschlecht höher, dies verändert sich ab dem Pubertätsalter bis zum Erwachsenenalter zu Lasten des weiblichen Geschlechts [35].

- Psychische Faktoren

Es existieren Hinweise, dass Asthma zu psychosozialen Belastungen führen kann, umgekehrt aber auch psychosoziale Belastungen das Risiko für die Entstehung von Asthma erhöhen können [36, 37]. Psychische Faktoren und Emotionsausdruck können Entzündungsprozesse, Lungenfunktion und Anfallshäufigkeit beinflussen [2,38-42]. Insbesondere bei Patienten mit unzureichender Asthmakontrolle und multifaktoriellen schweren Verlaufsformen spielt oft eine psychische Komponente eine Rolle [2,43]. Stress der Mutter während der Schwangerschaft wurde ebenfalls mit kindlichem Asthma und Atopie in Verbindung gebracht [44].

\subsubsection{Exogene Faktoren}

- Allergene

Außen- und Innenraumallergene können Auslöser von Asthmaexazerbationen bei allergischem Asthma sein, die Rolle der Allergene bei der Asthmaentstehung ist jedoch bislang unklar. Einerseits wird postuliert, dass Allergene Asthma direkt auslösen können [45], andererseits wird angenommen, dass eine vorbestehende Atemwegspathologie (,airway premodeling“) durch Allergene und eine allergische Entzün- 
dung (besonders im Zusammenspiel mit frühkindlichen Atemwegsinfekten) lediglich aggraviert wird [5, 46]. Der Zeitpunkt und das Ausmaß der Allergenexposition könnten einen Einfluss darauf haben, ob ein Allergen schädigend oder protektiv wirkt $[47,48]$. Frühe allergische Sensibilisierung und Allergenexposition sind Risikofaktoren für die Entwicklung eines persistierenden Asthmas [49]. Die genauen Ursachen, warum bestimmte Antigene oft zu Allergenen werden und andere Antigene nicht, sind bislang noch wenig verstanden [50].

- Infektionen und Umweltbedingungen Es ist bis heute ungeklärt, ob Infektionen ein Asthma auslösen, oder vielmehr (wie im Rahmen der sogenannten „Hygiene-Hypothese“ postuliert) einer Asthmaentstehung entgegenwirken. Die diesbezügliche Datenlage ist widersprüchlich [51]. Wiewohl Luftverschmutzungen ein Asthma aggravieren können, ist deren Bedeutung für die Entstehung von Asthma noch nicht geklärt [52]. Im Gegensatz dazu gilt als gesichert, dass ein Aufwachsen auf einem traditionellen Bauernhof vor der Entwicklung eines Asthmas schützt [5355]. Als mögliche Ursachen dafür werden Tierkontakte, das Trinken von unpasteurisierter Milch und die Exposition gegenüber Endotoxinen oder anderen Pathogen-assoziierten Molekülen diskutiert [55]. Die Diversität an Mikroorganismen (und nicht deren Quantität oder Pathogenität) scheint hierbei eine wichtige Rolle zu spielen („Biodiversitäts-Hypothese“) [56]. Expositionen mit Mikroorganismen könnten auch Ursache des Asthma-protektiven Effekts eines Aufwachsens mit älteren Kindern oder eines frühen Kindergartenbesuchs sein [57]. Respiratorische Virusinfektionen im Alter von unter 3 Jahren (vor allem durch Rhinoviren) sind mit einem höheren Risiko assoziiert [58], ein Asthma im späteren Leben zu entwickeln [59], möglicherweise setzt dieser Effekt aber eine vorbestehende allergische Sensibilisierung oder Disposition voraus [60]. Die Frage, welchen Einfluss mikrobielle Expositionen auf die Entstehung von Asthma haben, könnte u.a. mit sogenannten Toll-like-Rezeptoren (TLR), die für die Erkennung mikrobieller Antigene durch das angeborene Immunsystem verantwortlich zeichnen, in Zusammenhang stehen [9,61]. Der Einfluss des Mikrobioms der Atemwege auf den Schweregrad eines bereits etablierten Asthmas ist Gegenstand aktueller Forschungen [62].

- Berufliche Expositionen

Diese werden an anderer Stelle ausführlich beschrieben (siehe Kapitel „Arbeitsbedingtes Asthma“).

- Sonnenlicht Vitamin D-Mangel (definiert als < $30 \mathrm{ng} 25(\mathrm{OH}) \mathrm{D} 3 / \mathrm{ml} \mathrm{Se-}$ rum) findet sich bei über $50 \%$ der Bevölkerung in den westlichen Industrienationen [63]. Dies wird vor allem auf eine mangelnde Exposition mit ungefiltertem Sonnenlicht (aufgrund langer Aufenthalte in Innenräumen) zurückgeführt. Eine Reihe von Studien postuliert einen Zusammenhang zwischen Asthmaschweregrad und Vitamin D-Mangel [64, 65]. Es ist jedoch unklar, ob hier ein pathogenetischer Zusammenhang vorliegt [66], klinische Studien konnten bislang nicht nachweisen, dass eine Vitamin-D-Supplementie- rung die Asthmakontrolle verbessert [67] oder die Asthmaentstehung verhindert [68].

- Tabakrauch

Zigarettenrauchen vermindert die Wahrscheinlichkeit einer Asthmakontrolle und führt zu vermehrten Exazerbationen und zu einem verminderten Ansprechen auf inhalative oder systemische Glucocorticoide bei Patienten mit Asthma [6973]. Auch die passive Exposition mit Zigarettenrauch hat einen negativen Einfluss auf die Lungenfunktion, insbesondere in der frühen Kindheit und durch die Mutter während der Schwangerschaft [74]. Diese Einflüsse sind nicht auf eine erhöhte Allergieneigung unter Rauchexposition zurückzuführen [75].

- Ernährung und Medikamente

Die Zufuhr von Vitaminen, die Ernährung mit mediterraner Kost und mit Früchten und Gemüse ist mit einer erniedrigten Inzidenz von Asthma bzw. einer besseren Asthmakontrolle assoziiert, die klinische Bedeutung und der Mechanismus ist jedoch unklar [76,77]. Auch die mütterliche Ernährung während der Schwangerschaft ist hierbei von Bedeutung $[78,79]$. Ob das mütterliche Stillen einen protektiven Effekt für den Säugling bezüglich der Entstehung von Asthma hat, ist weiter umstritten [80]. Es gibt bislang keinen sicheren Anhalt dafür, dass der Einsatz von Antibiotika im Kindesalter die Entstehung von Asthma fördert [81]. Ob die Einnahme von Paracetamol in der Kindheit einen Einfluss auf die Asthmaentstehung hat, ist ebenfalls hypothetisch [82,83]. Bei Patienten mit Aspirin-Intoleranz (siehe Kapitel 3.3.5) führt die Einnahme von Hemmern der Cyclooxygenase-1 (COX-1) zu einer Zunahme der Asthmabeschwerden bis hin zu schwersten Exazerbationen [84].

\subsection{Asthmaformen}

Die Kriterien für die Diagnose eines Asthmas schließen jegliche Kombination von Atembeschwerden, variabler Atemwegsobstruktion, Atemwegsüberempfindlichkeit und entzündlichen Schleimhautveränderungen ein. Des Weiteren findet sich auch ein variables Ansprechen auf medikamentöse Therapien. Dies führt naturgemäß zu einem heterogenen Krankheitsbild mit vielen verschiedenen Phänotypen [85]. Im Gegensatz zu „Endotypen“ (Gruppen mit einem definierten Pathomechanismus) werden „Phänotypen“ als Ausprägungen der Erkrankung bezeichnet, welche sich durch klinische (wie z.B. das Vorhandensein einer Allergie) und/oder laborchemische (wie z. B. die Vermehrung von Bluteosinophilen) Merkmale voneinander abgrenzen lassen. Die Unterteilung in Phänotypen ist aus klinischer Sicht allerdings nur sinnvoll, wenn diese Phänotypen sich im Krankheitsverlauf unterscheiden oder sich Konsequenzen für die Therapie ergeben [86]. Folgende, sich teilweise überschneidende Phänotypen werden gegenwärtig diskutiert.

\subsubsection{Allergisches (extrinsisches) versus nicht allergisches (intrinsisches) Asthma}

Eine atopische Diathese, d.h. die Bereitschaft zur Produktion von IgE-Antikörpern gegen häufige Aeroallergene der Umwelt, ist der stärkste bislang identifizierte prädisponierende Faktor zur Asthmaentwicklung. Im Falle eines Nachweises von Sensibi- 
lisierungen gegen typische Allergene (z.B. im Pricktest oder durch Messung von spezifischem IgE im Serum) bei gleichzeitigem Allergenbezug der Asthmabeschwerden wird daher der Begriff allergisches bzw. extrinsisches Asthma verwandt. Eine erhöhte Gesamt-IgE-Konzentration im Serum ist (im Gegensatz zu erhöhten spezifischen IgE-Konzentrationen im Serum) wahrscheinlich ein eigenständiger Indikator, welcher nicht zwingend auf das Vorhandensein von Allergien hinweist [87] und unter anderem durch eine polyklonale IgE-Vermehrung infolge von Superantigen-Stimulationen bedingt sein kann [88]. Allergien sind insbesondere im Kindes- und Jugendalter von pathogenetischer Relevanz (und finden sich hier bei der Mehrheit der Patienten), im höheren Erwachsenenalter können jedoch auch Allergien eine Rolle spielen, z. B. bei beruflich bedingtem Asthma. Beim allergischen Asthma können saisonale und perenniale Verlaufsformen unterschieden werden. Typische klinische Beispiele für saisonal akzentuiertes Asthma sind Allergien gegen Baum- oder Gräserpollen sowie Schimmelpilzsporen (z. B. Alternaria) [89-91]. Das saisonale Asthma ist häufig mit einer allergischen Rhinitis bzw. Rhinokonjunktivitis assoziiert [92]. Die Patienten können außerhalb der saisonalen Allergenexposition völlig asymptomatisch sein und eine normale Lungenfunktion aufweisen. Andererseits kann sich ein saisonales Asthma auch als zeitlich limitierte Akzentuierung der Beschwerden präsentieren. Der Schweregrad eines saisonalen Asthmas variiert von Patient zu Patient und von Allergensaison zu Allergensaison, als Konsequenz der wechselnden Allergenkonzentration in der Atemluft. Vorangegangene Asthmaexazerbationen nach Allergenkontakt prädisponieren jedoch zu gehäuften Exazerbationen in späteren Jahren [93]. Häufige Ursachen eines perennialen allergischen Asthmas sind die Hausstaubmilbenallergie und Tierhaarallergie. Kinder mit einer Sensibilisierung gegen Hausstaubmilben oder Tierepithelien zeigen ein erhöhtes Risiko, später Asthma zu entwickeln [49].

Bei $30-50 \%$ der Erwachsenen mit Asthma sind weder anamnestisch noch mittels Hauttest oder Bestimmung von spezifischem IgE im Serum Allergien gegen Umweltallergene nachweisbar. Diese Form des Asthmas, die man als nicht allergisches oder intrinsisches Asthma bezeichnet (letzterer Begriff erscheint sinnvoller, da der fehlende Nachweis einer allergischen Sensibilisierung eine Allergie nicht ausschließt [94]), wird häufig durch Infektionen der Atemwege getriggert. Es finden sich immunpathologische Ähnlichkeiten zwischen extrinsischem und intrinsischem Asthma, so kann z. B. bei beiden Asthmaformen eine Eosinophilie in den Atemwegen vorliegen [95]. Mischformen sind möglich, insbesondere kann auch bei einem initial allergischen Asthma im Verlauf die intrinsische Komponente klinisch in den Vordergrund treten. Zudem kann trotz Nachweis einer spezifischen allergischen Sensibilisierung (im Hauttest oder in der Serologie) ein primär intrinsisches Asthma ohne Bezug zu dieser Sensibilierung vorliegen [96]. Die Unterscheidung in allergisches und intrinsisches Asthma ist von therapeutischer Bedeutung, da sich bei allergischen Asthmaformen spezifische Therapieoptionen ergeben können, wie die spezifische Immuntherapie (SIT) [97], die Allergenkarenz oder die Behandlung mit Biologika, die für Patienten mit Allergienachweis zugelassen sind [98].

\subsection{2 „Type-2-High“- versus „Type-2-Low“-Asthma}

Die Erkenntnis, dass bestimmte Zytokine wie IL-5 und IL-13 nicht nur von Allergen-spezifischen T-Helfer-Zellen, sondern auch von Allergen-unspezifischen „innate lymphoid cells“ (ILC2) freigesetzt werden können (siehe $>$ Tab.1), führte zu dem Konzept, beide Inflammationstypen zum Überbegriff „Type-2“- bzw. „Type-2-High“-Asthma zusammenzufassen [15, 99]. Klinisch bedeutsam könnte der Phänotyp eines „Type-2High"-Asthmas für das Ansprechen auf eine Therapie mit inhalativen Glucocorticosteroiden oder bestimmten Anti-Interleukin-Biologika sein. Umstritten ist jedoch, welcher klinische Biomarker (wie z.B. exhaliertes NO oder Periostin) ein „Type-2High"-Asthma am besten identifiziert und ob es gemeinsame genetische Signaturen für diesen Sammelbegriff gibt [99]. Des Weiteren ist umstritten, inwieweit ein „Type-2-Low“-Asthma überhaupt einem Asthma (welches typischerweise auf eine Glucocorticosteroid-Therapie anspricht) entspricht oder ob es eine eigenständige Atemwegserkrankung darstellt.

\subsubsection{Eosinophiles versus nicht eosinophiles Asthma}

Asthma kann anhand des Vorliegens einer eosinophilen Atemwegsentzündung in eosinophiles und nicht eosinophiles Asthma unterschieden werden [100]. Nicht eosinophiles Asthma kann weiter in neutrophile oder pauci-granulozytäre (kein Nachweis einer Granulozytenvermehrung) Subtypen unterschieden werden. Die Unterscheidung der Subtypen erfolgt vor allem anhand von Sputumuntersuchungen und anhand des Differenzialblutbildes [101]. Anhand von Sputumuntersuchungen wurde postuliert, dass bis zu $50 \%$ aller Patienten mit mildem bis moderatem Asthma ein nicht eosinophiles Asthma haben [102], wenngleich die Phänotypen im Zeitverlauf nicht immer stabil sind [103]. Untersuchungen des Differenzialblutbildes von über 130000 Patienten mit der Diagnose Asthma in Großbritannien erbrachten eine mediane Eosinophilenkonzentration von 200 Eosinophilen/ul Blut [18]. Ab welchem Grenzwert der Bluteosinophilen-Konzentration von einer „Blut-Eosinophilie" gesprochen werden kann, ist noch nicht abschließend geklärt. Würde man einen Grenzwert von 150 Eosinophilen/ $\mu$ l postulieren, wäre die Mehrheit „eosinophil“, würde man einen Grenzwert von 300 Eosinophilen/ $\mu$ l postulieren, wäre die Mehrheit „nicht eosinophil“. Unklar ist aktuell auch, inwiefern ein „eosinophiles“ Asthma mit einem „Type-2-High“-Asthma bzw. ein „nicht eosinophiles“ Asthma mit einem „Type-2-Low“-Asthma gleichzusetzen ist $[99,104]$. Therapeutisch bedeutsam ist, dass sich die Patienten mit eosinophilem und nicht eosinophilem Asthma in ihrem Ansprechen auf ICS deutlich unterscheiden [100]. Des Weiteren gewinnt diese Klassifikation eine zunehmende Bedeutung für die Indikationsstellung einer Biologikatherapie, da eine Eosinophilenvermehrung das Ansprechen auf bestimmte Biologika wahrscheinlicher macht (wie Anti-IgE, Omalizumab [105], oder Anti-IL-4-Rezeptor alpha, Dupilumab [106]) oder überhaupt erst ein Ansprechen voraussagt (wie bei den Anti-IL-5-Biologika Mepolizumab, Reslizumab und Benralizumab [107-110]). 


\subsubsection{Cough-variant-Asthma („Husten als Asthma- Äquivalent")}

Eine Gruppe von Patienten, bei denen die Asthmadiagnose häufig nicht oder erst spät gestellt wird, sind Patienten mit einem "Cough-variant-Asthma“ (Cough-Type-Asthma, Husten als Asthma-Äquivalent) [111, 112]. Diese Patienten weisen einen chronischen trockenen Husten auf, dessen Ursache zunächst unklar ist. Es besteht eine unspezifische bronchiale Hyperreagibilität, weitere typische Symptome eines Asthmas (z. B. Atemnot, Giemen und Brummen) fehlen dagegen. Die Lungenfunktion und die Röntgenaufnahme der Thoraxorgane sind normal. Husten als Asthma-Äquivalent ist eine häufige Ursache des chronisch persistierenden Hustens. Differenzialdiagnostisch abzugrenzen sind davon Husten bei Behandlung mit Inhibitoren des Angiotensin-konvertierenden Enzyms (ACE-Inhibitoren), Husten bei Patienten mit gastroösophagealem Reflux (GERD) oder Erkrankungen der oberen Atemwege (z. B. chronische Rhinitis, Sinusitis, Pharyngitis, Laryngitis) und chronischer idiopathischer Husten mit Erhöhung der Sensitivität des Hustenreflexes [113]. Ein Drittel der Patienten mit Cough-Variant-Asthma entwickelt im weiteren Verlauf ein klassisches Asthma. Patienten mit Cough-Variant-Asthma sprechen gut auf klassische anti-asthmatische Therapieoptionen an, eine frühzeitige Therapie kann den Übergang in ein klassisches Asthma verhindern [111].

\subsubsection{Sonstige Asthmaformen}

Asthma bei Aspirin-Intoleranz („Aspirin-exacerbated airway disease: AERD“) Patienten mit Asthma bronchiale, die eine nasale Polyposis und eine chronische Sinusitis aufweisen, können eine Intoleranz gegenüber Hemmern der Cyclooxygenase-1 (COX-1) wie Acetylsalicylsäure (ASS) oder Ibuprofen aufweisen [84]. Es wird vermutet, dass diese pharmakologische Intoleranz eine vorbestehende Schädigung der Atemwege lediglich aggraviert, nicht jedoch auslöst, daher wird derzeit der Begriff „Aspirin-exacerbated respiratory disease“ favorisiert. Die genaue Prävalenz ist unklar, Angaben schwanken zwischen 5,5\% und 12,4\% der Asthmapatienten [114]. Bei schwereren Asthmaformen ist die AERD-Prävalenz höher (14,9\%) [114]. Frauen sind häufiger als Männer betroffen, es handelt sich überwiegend um intrinsisches Asthma [84]. Bei der AERD gehen die Rhinosinusitis und die Polyposis nasi der Asthmaentstehung meist voraus, der typische Erkrankungsbeginn liegt zwischen dem 20. und 40. Lebensjahr. Die Pathogenese dieser Asthmaform ist bis heute unklar, eine Dysregulation pro- und antiinflammatorischer Lipidmediatoren mit einer gesteigerten Endorgan-Sensitivität gegenüber diesen Mediatoren scheint jedoch eine Rolle zu spielen [115]. Die Identifizierung dieses Asthmasubtyps ist von therapeutischer Bedeutung, da eine Dauertherapie mit ASS („Adaptive Desaktivierung") sowohl die Polyposis und Sinusitis als auch das Asthma deutlich bessern kann [84].

Asthma und körperliche Belastung Wenn Atemwegsobstruktionen ausschließlich bei Belastung auftreten, wird in der Literatur der Begriff "Anstrengungs-induzierte Bronchokonstriktion“ („Exercise-induced Bronchoconstriction, ElB“) empfohlen [116]. Bei Menschen, die Ausdauersportarten betreiben (inbesondere Hochleistungssportarten mit hohen Atemminutenvolumina), kommt es gehäuft zur Entwicklung eines Asthmas (welches sich entweder nur bei Anstregung manifestiert oder als klassisches Asthma imponiert). Als Ursache wird eine wiederholte Austrocknung der Schleimhäute, die ein Remodeling der Atemwege und die Entwicklung einer bronchialen Hyperreagibilität fördert, postuliert [117]. Bei behandeltem Asthma kann das Auftreten von anstrengungsinduzierter Bronchialobstruktion Hinweis auf eine nicht ausreichende Asthmakontrolle sein. Die Asthmatherapie von Leistungssportlern (die sich grundsätzlich nicht von der klassischen Asthmatherapie unterscheidet) ist durch die Besonderheit gekennzeichnet, dass die Behandlung mit Beta-2-Sympathomimetika den Regularien der WADA (World Anti-Doping Association) unterliegt [117]. Informationen über die aktuellen Regularien sind bei den nationalen Anti-Doping-Agenturen Deutschlands [118], Österreichs [119] und der Schweiz [120] zu erfahren.

Asthma bei älteren Patienten Bei älteren Patienten wird die Diagnose eines Asthmas oft nicht gestellt oder übersehen, da die Wahrscheinlichkeit der Erstdiagnose Asthma mit zunehmendem Alter abnimmt, die Lungenfunktion im Alter natürlicherweise abnimmt, die Perzeption der Beschwerden im Vergleich zu jüngeren Patienten häufig inadäquat ist und ältere Patienten oft Schwierigkeiten mit der Mitarbeit bei Lungenfunktionsprüfungen haben. Zudem ist die Abgrenzung von einer chronisch-obstruktiven Lungenerkrankung (COPD) schwierig, insbesondere von einer COPD bei Nierauchern ( $>10 \%$ aller COPD-Patienten über 70 Jahre [121]). Erschwert wird dies durch den Umstand, dass unterschiedliche Obstruktionsdefinitionen ( $\mathrm{FEV}_{1} \% \mathrm{VC}<70 \%$ versus $\mathrm{FEV}_{1} \% \mathrm{VC}<$ der unteren Altersnorm, dem sogenannten „Lower Limit of Normal“) [122] im höheren Alter zu unterschiedlichen Bewertungen der Lungenfunktion führen können. Dennoch ist ein undiagnostiziertes Asthma eine nicht seltene und behandelbare Ursache von Atembeschwerden bei älteren Menschen, der zu wenig Aufmerksamkeit geschenkt wird [123]. Hinweisend auf ein Asthma im höheren Alter sind (in Abgrenzung von der COPD) erhöhte Gesamt-IgE-Spiegel, eine Blut-Eosinophilie und eine nächtliche Akzentuierung der Beschwerden. Die Unterscheidung eines Asthmas von einer COPD bei älteren Menschen ist wichtig, da eine Monotherapie mit inhalativen Beta-2-Sympathomimetika als Dauertherapie (die bei der COPD sicher und effektiv ist) bei Asthma die Symptomatik und Mortalität steigern kann $[22,123]$.

\section{Asthma und Sensibilisierung gegen Pilzantigene Sensibili-} sierungen gegen Pilzantigene können Mitursache eines schweren Asthmas sein [124]. Dies gilt insbesondere für die allergische bronchopulmonale Aspergillose (ABPA), die neben einer Sensibilisierung gegen Aspergillus fumigatus durch hohe Gesamt-IgESpiegel $(>1000 \mathrm{IU} / \mathrm{ml})$, pulmonale Infiltrate und zentrale Bronchiektasien gekennzeichnet ist und gut auf eine hochdosierte Glucocorticoidtherapie anspricht. Umstritten hingegen ist aktuell, ob das alleinige Vorliegen einer Sensibilisierung gegen diverse Pilzantigene ohne hohe Gesamt-IgE-Spiegel („Severe asthma with Fungal Sensitization: SAFS“) bereits einen abgrenzbaren Phänotyp des schweren Asthmas darstellt, und ob eine antimykotische Therapie in diesem Falle sinnvoll ist [125]. 


\subsection{Schweres Asthma}

Es ist bislang umstritten, ob das sogenannte schwere Asthma („severe asthma“) eine eigene Asthmaform darstellt, oder ob es sich lediglich um schwere Verlaufsformen der oben genannten Asthmaphänotypen handelt [126]. Die Weltgesundheitsorganisation (WHO) unterscheidet das schwere Asthma in $3 \mathrm{Un}$ tergruppen [127]:

- unbehandeltes schweres Asthma („untreated severe asthma"): bedingt durch fehlende medikamentöse Therapie

- schwierig zu behandelndes Asthma („difficult-to-treat asthma“): bedingt durch Incompliance, inkorrekte Medikamenteneinnahme, Komorbidität oder mangelnde Elimination von Triggern

- therapierefraktäres schweres Asthma („treatment-resistant severe asthma“): fehlende Asthmakontrolle trotz korrekter Einnahme leitliniengerechter Basistherapeutika in höherer Dosis.

Gemäß Konsensusempfehlung der internationalen Fachgesellschaften (ERS/ATS 2014) ist schweres Asthma dadurch definiert, dass unter hochdosierter inhalativer Glucocorticoidtherapie (in Verbindung mit einem zweiten „Controller“ wie z. B. mit langwirksamen Beta-2-Sympathomimetika) oder systemischer Glucocorticoidtherapie keine ausreichende Asthmakontrolle erreicht wird $(A C T<20)$ bzw. wiederholte Exazerbationen auftreten oder eine erhebliche Atemwegsobstruktion persistiert $[127,128]$. Diese Definition sieht somit unbehandeltes schwergradiges Asthma (WHO-Klasse I) nicht als „schweres Asthma“ an, schließt hingegen bestimmte Formen des schwierig zu behandelnden Asthmas (WHO-Klasse II) durchaus ein. Die aktuelle Definition einer „hochdosierten inhalativen Glucocorticoidtherapie“, welche für die Definition von schwerem Asthma von zentraler Bedeutung ist, ist bislang nicht einheitlich: der Konsensus der internationalen Fachgesellschaften (ERS/ATS 2014 [128]) ( $\triangleright$ Tab.9) gibt höhere Dosierungen vor als die aktuelle GINA-Empfehlung (2017) [2] ( $\triangleright$ Tab.8). Die seitens des ERS/ ATS-Konsensus vorgeschlagenen Dosen ( $>$ Tab.9) erscheinen bei Erwachsenen sinnvoller, da sie den zugelassenen Maximaldosen und der klinischen Realität am ehesten entsprechen. Bevor die Diagnose eines schweren Asthmas gestellt wird, gilt es jedoch zu überprüfen, inwieweit es sich überhaupt um ein Asthma oder aber eine andere Erkrankung handelt [127].

\subsection{Epidemiologie und ökonomische Bedeutung}

Die Prävalenzdaten bezüglich der durch einen Arzt gestellten Diagnose Asthma sind weltweit sehr unterschiedlich, die Zahlen schwanken zwischen 1,8\% in Vietnam und 32,8\% in Australien [129]. Inwieweit dies die tatsächliche Prävalenz von Asthma wiedergibt, ist unklar. In Deutschland ist Asthma eine der häufigsten chronischen Erkrankungen, die bei ca. 10\% der kindlichen [130] und ca. 5\% der erwachsenen Bevölkerung vorkommt [131]. Die Prävalenz von Asthma hatte in den vergangenen Jahrzehnten in vielen Ländern zugenommen (inbesondere bei Kindern und Jugendlichen), in den letzten Jahren scheint die Zunahme der Asthmaprävalenz in westlichen Ländern zum Stillstand gekommen zu sein [132]. Aussagekräftige Studien zum Asthma im hohen Lebensalter fehlen. Die Prävalenz von Asthma ist in den hochentwickelten westlichen Ländern höher als in sogenannten Entwicklungs- und Schwellenländern, in den letzten Jahren nähern sich die Prävalenzraten jedoch einander an [133]. Die volkswirtschaftliche Bedeutung von Asthma ist groß. In einer gesamtwirtschaftlich ausgerichteten Krankheitskosten-Analyse aus dem Jahr 1996 wurde ein Gesamtbetrag von 2,6 Milliarden Euro pro Jahr in Deutschland ermittelt [134], durch die gestiegene Prävalenz der Erkrankung und die Einführung neuer Therapieoptionen könnte dieser Betrag aktuell noch höher liegen. Krankheitskosten für Kinder und Erwachsene mit mittelschwerem allergischem Asthma wurden mit 2200 bzw. 2700 Euro pro Patient pro Jahr berechnet, bei schwerem allergischem Asthma resultierten 7900 bzw. 9300 Euro pro Patient und Jahr [135]. Die Kosten für die vielfach schwereren Krankheitsverläufe nicht allergischer Asthmaformen dürften höher anzusetzen sein.

\subsection{Verlauf der Erkrankung und Mortalität}

Mehr als die Hälfte der Menschen, bei denen im Kindesalter ein Asthma diagnostiziert wurde, haben im Erwachsenenalter keine Asthma-typischen Symptome mehr (transiente Verlaufsform) [35]. Dies erklärt die deutlichen Unterschiede in der Asthmaprävalenz bei Kindern und Erwachsenen. Bei unbehandeltem und persistierendem Asthma, insbesondere bei fehlender oder unzureichender antiinflammatorischer Therapie, ist mit zunehmender Krankheitsdauer zu beobachten, dass eine fixierte Atemwegsobstruktion mit anhaltenden, nicht mehr oder wenig reversiblen Beschwerden und einem akzelerierten Abfall der Lungenfunktion auftritt [136]. Bei einer adäquaten Therapie ist die Lebenserwartung der Asthmatiker vergleichbar derjenigen gesunder Menschen, die Nebenwirkungen einer adäquaten Therapie (vor allem der Therapie mit inhalativen Glucocorticoiden) sind im Verhältnis dazu gering [137]. Die Asthmamortalität in Deutschland hat in den letzten Jahren dank der verbesserten therapeutischen Optionen und Versorgung abgenommen. Die Abnahme der Mortalität betrifft alle Altersgruppen einschließlich der kindlichen Asthmatodesfälle. Diese Entwicklung wird im Wesentlichen der Therapie mit inhalativen Glucocorticoiden zugeschrieben [138]. Die durch das statistische Bundesamt veröffentlichten, auf Totenschein-Diagnosen beruhenden Asthmasterbefälle (Jahr 2008: 1500 Tote) überschätzen wahrscheinlich die reale Anzahl an Asthma-Toten in Deutschland erheblich. Indiz dafür ist der Umstand, dass die große Mehrheit der berichteten „Asthma-Toten“ unter geriatrischen Patienten zu finden ist [139]. 


\section{EMPFEHLUNGEN}

E1 Die Diagnose Asthma bronchiale soll anhand der Anamnese, charakteristischer Symptome und der Befunde der Lungenfunktionsprüfungen gestellt werden.

E2 Bei jungen Kindern, die zur Kooperation bei der Lungenfunktionsprüfung noch nicht in der Lage sind, ist die Diagnostik deutlich erschwert. Neben familien- und eigenanamnestischen Hinweisen auf atopische Erkrankungen sollen gezielt erfragt/getestet werden:

- $\geq 3$ asthmatypische Episoden im letzten Jahr

- stationärer Aufenthalt wegen Symptomatik einer Atemwegsobstruktion

- giemende Atemgeräusche/trockener Husten ohne Infekt, insbesondere bei körperlicher Anstrengung

- Ansprechen der Symptome auf einen Therapieversuch mit antiasthmatisch wirkenden Medikamenten

- Nachweis einer Sensibilisierung auf Allergene

E3 Nur bei Patienten mit charakteristischen Symptomen und einer manifesten, voll reversiblen Bronchialobstruktion (oder einer im Verlauf variablen Obstruktion mit zeitweiliger Normalisierung) soll die Diagnose des Asthmas als gesichert gelten. Bei Patienten mit initial reversibler, aber im Verlauf fixierter Atemwegsobstruktion besteht die Diagnose „Asthma“ weiter.

E4 Im Kindesalter kann auch bei numerisch „normalen“ Lungenfunktionswerten eine konkave Deformierung des exspiratorischen Schenkels der Fluss-Volumen-Kurve, die sich im Bronchodilatationstest als reversibel erweist, auf ein behandlungsbedürftiges Asthma hinweisen. Darüber hinaus schließt eine normale Ruhelungenfunktion ein behandlungsbedürftiges Asthma nicht aus.
E5 Bei Patienten mit charakteristischen Symptomen ohne Bronchialobstruktion ebenso wie bei fixierter, unter antiasthmatischer Therapie nicht vollständig reversibler Bronchialobstruktion sollte die Diagnose durch Verlaufsuntersuchungen und/oder durch Nachweis einer bronchialen Hyperreagibilität und/oder durch Nachweis der Variabilität der eine Bronchialobstruktion anzeigenden Parameter (z. B. $\mathrm{FEV}_{1}$, Peak flow) wahrscheinlich gemacht werden.

E6 Der Nachweis einer bronchialen Hyperreagibilität ohne charakteristische Symptome soll nicht als hinreichend für die Diagnose Asthma gelten, da auch Gesunde bronchial hyperreagibel sein können.

E7 Bei Patienten mit intermittierenden charakteristischen Symptomen ohne aktuelle Bronchialobstruktion mit negativem Provokationstest und ohne Nachweis der Variabilität der eine Bronchialobstruktion anzeigenden Parameter sollte die Diagnose Asthma als unwahrscheinlich, bei uncharakteristischen Symptomen als ausgeschlossen gelten.

E8 Im Rahmen der Diagnostik eines Asthmas sollen ein Allergietest (Hauttest und/oder Bestimmung von spezifischem IgE im Serum) durchgeführt sowie Gesamt-IgE im Serum und die Zahl der eosinophilen Granulozyten im Blut bestimmt werden.

S1 In der täglichen Praxis wird Asthma sowohl unter-als auch überdiagnostiziert.

S2 Das Vorliegen einer (allergischen) Rhinitis (Komorbidität) erhöht die Wahrscheinlichkeit der Diagnose Asthma.

\section{Diagnose des Asthmas}

Die Diagnose des Asthmas beruht auf 4 Säulen:

- Anamnese, Symptome und körperliche Untersuchungsbefunde

- variable und reversible Atemwegsobstruktion

- chronische Entzündung der Atemwege

- Überempfindlichkeit der Atemwege (bronchiale Hyperreagibilität).

\subsection{Anamnese}

\subsubsection{Erwachsene}

Die Asthmadiagnose wird durch folgende Angaben gestützt:

- Die Symptome (Husten, Giemen, Luftnot und Brustenge) treten allein oder gemeinsam wiederholt auf.

- Die Symptome verschlechtern sich während der Nacht und/oder in den frühen Morgenstunden.

- Die Symptome variieren in Zeit und Intensität und können ggf. nur episodenhaft auftreten. Längere Beschwerdefreiheit ist möglich, beispielsweise bei saisonal oder intermittierend auftretenden Allergenen oder Noxen.

- Die Symptome können durch virale Infekte der Atemwege, körperliche Belastung, Allergenexposition, Witterungsänderungen sowie private, öffentliche und berufliche Noxen ausgelöst werden.

Anamnestische Angaben und Befunde, die für die Diagnose eines Asthmas sprechen:

- Atopie (Neurodermitis, allergische Rhinitis) in der Eigenanamnese

- Atopie in der Familienanamnese

- Symptome im Bereich der oberen Atemwege sind häufig und auch bei Patienten mit nicht allergischem Asthma [96] zu berücksichtigen

Anamnestische Angaben, die eher gegen das Vorliegen eines Asthmas sprechen

- chronischer Husten und Auswurf ohne weitere respiratorische Symptome

- nur inspiratorisch empfundene Luftnot 


\subsubsection{Kinder}

Klassischerweise liegen bei Kindern anamnestisch wiederholte Episoden von erschwerter Atmung und Atemnot vor, oft begleitet von trockenem Reizhusten und geräuschvoller Ausatmung insbesondere bei oder nach körperlicher Belastung (z.B. auch Spielen oder Lachen). Es sollten bei jeder Anamneseerhebung bei Verdacht auf Asthma bronchiale aktiv nach Beschwerden bei körperlicher Aktivität oder Sport gefragt und allergologische Befunde erhoben werden. Je jünger die Kinder, desto schwieriger ist es, die Diagnose Asthma zu stellen. Viele Kleinkinder machen rezidivierende obstruktive Bronchitiden durch. Die meisten dieser rezidivierenden obstruktiven Bronchitiden sind rein Virusinfekt-assoziiert, und die Symptomatik verschwindet nach dem dritten Lebensjahr. Rezidivierend auftretende obstruktive Bronchitiden können aber auch Ausdruck eines frühkindlichen Asthmas sein. Anamnestische Hinweise darauf wären eine giemende Atmung ohne Vorliegen eines Infekts, z.B. bei körperlicher Anstrengung, oder eine positive Atopieanamnese [140-142].

Die wichtigsten Asthmasymptome sind

- Atemnot

- exspiratorische Atemnebengeräusche (Giemen und Brummen, pfeifende Atmung), ggf. Distanzgiemen im Exspirium

- trockener Husten ohne Infekt, bei oder nach körperlicher Belastung

\subsection{Klinische Untersuchungsbefunde}

Der körperliche Untersuchungsbefund unterscheidet sich entsprechend des aktuellen Schweregrades der Erkrankung. Bei einem leichtgradigen Asthma bronchiale kann der Untersuchungsbefund abhängig vom aktuellen Status regelrecht sein, da der Patient tagsüber untersucht wird, aber seine Beschwerden nachts oder in den frühen Morgenstunden vorhanden sind. Der Nachweis exspiratorischer Nebengeräusche ist bei forcierter Exspiration häufiger als bei Ruheatmung.

Bei schwergradigem Asthma sind die pathologischen Nebengeräusche häufiger. Der Klopfschall ist meist hypersonor aufgrund der Lungenüberblähung. Bei sehr schwerem Asthma können die Atemgeräusche kaum noch hörbar sein, da keine ausreichende Strömung in den Atemwegen aufgebracht werden kann („silent chest“). Prinzipiell können Atemnot, Husten und ein pathologischer Auskultationsbefund auch durch andere pulmonale (Atemwegsinfekte, COPD, Bronchiektasen) und kardiovaskuläre Erkrankungen (Herzinsuffizienz) bedingt sein.

Bei Kindern ist insbesondere auf folgende Symptome zu achten: Atopiezeichen, Zunahme des sagittalen Thoraxdurchmessers bei Überblähung, thorakale Einziehungen (jugulär, intercostal, epigastrisch) bei akuter Atemnot.

\subsection{Lungenfunktionsdiagnostik}

Für die Diagnose des Asthmas ist die Messung der Lungenfunktion erforderlich [2]. Sie dient sowohl dem Nachweis eines regelrechten Befundes als auch der Objektivierung einer Atemwegsobstruktion und ihrer Reversibilität und Variabilität. Eine normale Lungenfunktion schließt ein Asthma nicht aus. Der Nachweis des variablen Charakters der Atemwegsobstruktion erfordert serielle Lungenfunktionsmessungen oder den häuslichen Einsatz eines Peak-flow-Meters.

\subsubsection{Spirometrie}

Zur Durchführung, Qualitätskontrolle und Bewertung der Spirometrie darf auf die Empfehlungen zur Spirometrie der Deutschen Atemwegsliga verwiesen werden [122].

Spirometrische Messparameter Die wichtigsten Messwerte sind die forcierte Vitalkapazität (FVC), die Einsekundenkapazität $\left(\mathrm{FEV}_{1}\right)$ und das Verhältnis $\mathrm{FEV}_{1} / \mathrm{FVC}$ (Tiffeneau-Index). Die Messgrößen des Flussvolumendiagramms gehen nicht in die Beurteilung der spirometrischen Messung ein. Die qualitative Bewertung des Flussvolumendiagramms ist jedoch hilfreich für die Qualitätskontrolle der Durchführung der Messung.

Qualitätskontrolle Es sollen mindestens drei akzeptable Versuche aufgezeichnet werden. Die Differenz zwischen dem größten und zweitgrößten Wert für $\mathrm{FEV}_{1}$ und $\mathrm{FVC}$ soll nicht mehr als $5 \%$ und weniger als $150 \mathrm{ml}$ betragen.

Normalwerte Die Global Lung Initiative (GLI) hat 2012 spirometrische Referenzwerte für gesunde Probanden im Alter von 3 bis 95 Jahren erstellt. Im Gegensatz zu den bisher verwendeten Normwerten berücksichtigt GLI die Ethnie, die höhere Streuung der Messgrößen und damit eine niedrigere untere Normwertgrenze (5. Perzentile, LLN, lower limit of normal) im Alter sowie eine höhere $\mathrm{FEV}_{1} /$ FVC-Ratio (Tiffeneau-Index) bei Kindern und Jugendlichen, um eine Über- oder Unterschätzung der Obstruktion zu vermeiden [143]. Die Normalwerte basierend auf GLI sollten in die Software der Messgeräte integriert sein.

Schweregrade der Obstruktion Eine obstruktive Ventilationsstörung liegt vor, wenn $\mathrm{FEV}_{1} / \mathrm{FVC}<\mathrm{LLN}$ ist. Der Schweregrad der Obstruktion (nicht zu verwechseln mit dem klinischen Schweregrad des Asthmas) wird bei Erwachsenen als leicht bewertet bei $\mathrm{FEV}_{1} \geq 85 \%$ LLN (>60\% Soll), mittelschwer bei $\mathrm{FEV}_{1}$ Werten zwischen $55 \%$ und $84 \%$ LLN (40-60\% Soll) und schwer bei $\mathrm{FEV}_{1}<55 \%$ LLN ( $<40 \%$ Soll). Der Nachweis einer obstruktiven Ventilationsstörung ist mit der Diagnose eines Asthmas vereinbar, differenzialdiagnostisch müssen andere Erkrankungen, die mit einer obstruktiven Ventilationsstörung einhergehen, aber ggf. ausgeschlossen werden (siehe $>$ Tab. 2).

Besonderheiten des Kindesalters: Die Spirometrie kann unabhängig vom Schweregrad des Asthmas normal sein. Eine schematische Einteilung einer obstruktiven Ventilationsstörung in Schweregrade ist nicht üblich. Zur Beurteilung sollte eher der z-Wert für den jeweiligen Parameter $\left(\mathrm{FEV}_{1}, \mathrm{FEV}_{1} / \mathrm{FVC}\right)$ herangezogen werden. Der z-Wert gibt an, um wie viele Standardabweichungen ein Messwert vom Soll-Mittelwert abweicht, ein z-Wert von - 1,645 entspricht LLN [143]. 
Tab. 2 Häufige Differenzialdiagnosen des Asthmas bei Erwachsenen.

\begin{tabular}{|c|c|}
\hline $\begin{array}{l}\text { Krankheiten mit } \\
\text { obstruktiver } \\
\text { Ventilationsstörung }\end{array}$ & $\begin{array}{l}\text { Erkrankungen ohne regelhaft } \\
\text { vorliegende obstruktive } \\
\text { Ventilationsstörung }\end{array}$ \\
\hline $\begin{array}{l}\text { - COPD } \\
\text { - Bronchiektasen } \\
\text { - Mukoviszidose } \\
\text { - vocal cord dysfunction } \\
\text { (VCD) } \\
\text { - Lungenstauung mit } \\
\text { Obstruktion (Asthma } \\
\text { cardiale) } \\
\text { - zentrale Atemwegsstenose } \\
\text { (Tumoren, Tracheomalazie, } \\
\text { Fremdkörper) }\end{array}$ & $\begin{array}{l}\text { - chronische Bronchitis (nach } \\
\text { WHO-Definition) - chronisch } \\
\text { persistierender Husten } \\
\text { - rezidivierende Lungenembolie } \\
\text { - Sarkoidose } \\
\text { - Pneumothorax } \\
\text { - diffuse Lungenparenchym- } \\
\text { erkrankungen } \\
\text { - eosinophile Bronchitis } \\
\text { - Bronchiolitis } \\
\text { - Lungenstauung } \\
\text { - Hyperventilationssyndrom } \\
\text { - vorübergehende postinfektiöse } \\
\text { bronchiale Hyperreagibilität } \\
\text { (mit Husten) }\end{array}$ \\
\hline
\end{tabular}

\section{Spirometriebefunde, die gegen die Diagnose eines Asthmas sprechen:}

- normale Spirometrie bei Patienten mit akuten Symptomen

- immer normale Spirometrie im Langzeitverlauf der Erkrankung Erwachsener

- restriktive Ventilationsstörung mit normaler oder hoher $\mathrm{FEV}_{1} /$ FVC-Ratio (Tiffeneau- Index). Eine fehlende unspezifische bronchiale Hyperreagibilität (Definition, Testung siehe unten) macht die Diagnose des Asthmas unwahrscheinlich (siehe $>$ Abb. 1).

Reversibilitätstest mit Bronchodilatatoren Sofern eine obstruktive Ventilationsstörung vorliegt, sollte die Durchführung eines Reversibilitätstestes erfolgen. Eine Reversibilitätstestung kann auch bei einer „normalen“ Lungenfunktion sinnvoll sein, da der persönliche Bestwert trotz des im Normbereich liegenden Wertes deutlich höher sein kann [144].

Die Messungen erfolgen vor und 15 Minuten nach Inhalation eines kurzwirksamen Beta-2-Sympathomimetikums (SABA, bis zu $400 \mu \mathrm{g}$ Salbutamol in 4 separaten Dosen). Ein Reversibilitätstest kann prinzipiell auch mit einem kurzwirksamen Anticholinergikum (SAMA, $160 \mu \mathrm{g}$ Ipratropiumbromid) oder mit einer Kombination von SABA und SAMA durchgeführt werden. Ein positiver Reversibilitätstest liegt bei einem Anstieg der FEV um $>12 \%$ des Ausgangswertes und einem Anstieg um $200 \mathrm{ml}$ bei Erwachsenen vor.

Nur eine weitgehende Normalisierung der Lungenfunktion sichert die Asthmadiagnose. Ein positiver Reversibilitätstest entweder spontan oder nach Inhalation eines Bronchodilatators (siehe oben) macht die Diagnose eines Asthmas wahrscheinlich, ist aber nicht geeignet, eine COPD auszuschließen. In gleicher Weise deutet ein negativer Reversibilitätstest auf eine zum Untersuchungszeitpunkt ,fixierte' obstruktive Ventilationsstörung hin, ist aber nicht zur Differenzierung zwischen Asthma und COPD geeignet.
Bei Kindern werden zur Reversibilitätstestung ebenfalls in der Regel bis zu 4 Hübe eines SABA-Dosieraerosols (z. B. Salbutamol à $100 \mu \mathrm{g}$ ) verabreicht. Eine $\mathrm{FEV}_{1}$-Zunahme um $>12 \%$ gilt als positiv; das Kriterium der geforderten Zunahme um $200 \mathrm{ml}$ absolut ist angesichts der kleineren Volumina in der Regel nicht anwendbar. Immer ist zunächst die Form der Fluss-VolumenKurve zu beurteilen: Auch bei numerisch nicht ausreichender $\mathrm{FEV}_{1}$-Zunahme kann eine Normalisierung eines primär konkav deformierten exspiratorischen Schenkels auf eine klinisch relevante Reversibilität der obstruktiven Ventilationsstörung hinweisen. Bei Kleinkindern, die noch keine Spirometrie durchführen können, kann der Nachweis der Reversibilität ggf. - wenn primär Nebengeräusche vorlagen - auskultatorisch erfolgen. Alternativ kann als weniger mitarbeitsabhängige Methode der spezifische Atemwegswiderstand gemessen werden, hier gilt eine Abnahme um $>50 \%$ als signifikant.

Reversibilitätstestung unter Therapie mit Glucocorticosteroiden Die Reaktion der $\mathrm{FEV}_{1}$ auf inhalative Glucocorticosteroide in einer stabilen Phase der Erkrankung kann bei Erwachsenen durch eine zweimal tägliche Inhalation einer hohen ICSDosis über mindestens 4 Wochen geprüft werden. Bei Kindern kann das Ansprechen auf eine mehrwöchige Therapie mit ICS (6-8 Wochen) als Diagnosekriterium ebenfalls herangezogen werden. Die Kriterien für ein Ansprechen der FEV $\mathrm{F}_{1}$ und die Interpretation entsprechen denjenigen bei der Applikation von Bronchodilatatoren. Alternativ ist die Durchführung eines Reversibilitätstests auch mit systemischen Glucocorticosteroiden (20 - 40 mg Prednisolon/Tag oral für 7 - 14 Tage) möglich.

- Eine stets fehlende oder geringe Reversibilität der Obstruktion im akuten Test und im Verlauf trotz adäquater Behandlung spricht gegen die Diagnose eines Asthmas.

Messung der Überempfindlichkeit der Atemwege Die Überempfindlichkeit der Atemwege ist ein charakteristisches Merkmal des Asthmas. Sie kann jedoch auch bei allergischer Rhinitis, Mukoviszidose, COPD, Sarkoidose und bei Gesunden [145] nachweisbar sein. Die Messung der Überempfindlichkeit der Atemwege ist bei entsprechendem Verdacht sinnvoll, sofern die Anamnese mit einem Asthma vereinbar ist, jedoch keine klinischen Zeichen und/oder eine obstruktive Ventilationsstörung nachweisbar sind [146, 147].

Die Überempfindlichkeit der Atemwege kann mit verschiedenen Stimuli gemessen werden. Körperliche Belastung, Kaltluftprovokation, Inhalation mit Mannitol, hyper- und hypotoner Kochsalzlösung sowie Adenosin werden als indirekte Stimuli bezeichnet, da durch sie entzündliche Mediatoren freigesetzt werden, die eine Bronchokonstriktion auslösen [148].

Die Messung der Überempfindlichkeit der Atemwege durch Inhalation von Methacholin oder Histamin erzeugt eine Kontraktion der Atemwegsmuskulatur und wird daher als direkte Stimulation bezeichnet. Die Sensitivität und Spezifität der Verfahren unterscheidet sich. Im klinischen Alltag hat sich die Messung der Überempfindlichkeit der Atemwege mit Methacholin durchgesetzt.

Kriterien eines positiven Tests sind ein $\mathrm{FEV}_{1}$-Abfall ( $\geq 20 \%$ ) bzw. eine Verdopplung des spezifischen Atemwegswiderstan- 
des ( $\geq 2,0 \mathrm{kPa} \times \mathrm{sec}$ ) (siehe Empfehlungen zur Ganzkörperplethysmografie [149]).

Es gibt keine einheitliche Empfehlung, welche Veränderung der FEV $\mathrm{V}_{1}$ nach körperlicher Belastung (in der Regel standardisierte Laufbelastung) signifikant ist. Bei Erwachsenen wird ein Cut-off von $10 \%$ empfohlen [117], bei Kindern abhängig von der Untersuchungssituation $10-15 \%$ [150].

Gerade bei Kindern mit anstrengungsassoziierten Beschwerden wird die Messung der bronchialen Überempfindlichkeit mittels Lauf-Belastungs-Test zur Objektivierung der angegebenen Symptome empfohlen [151].

\subsubsection{Ganzkörperplethysmografie}

Die Ganzkörperplethysmografie erlaubt die Bestimmung des spezifischen Atemwegswiderstandes und des intrathorakalen Gasvolumens und eignet sich zur Objektivierung der Lungenüberblähung. Überdies ist die qualitative Beurteilung der inspiratorischen und exspiratorischen Atemwegswiderstände möglich. Dieses Vorgehen ist besonders zur funktionellen Differenzialdiagnose zwischen Asthma und COPD hilfreich. Ausführliche Empfehlungen zur Ganzkörperplethysmografie liegen vor [149].

Während gut angeleitete Kindergartenkinder oftmals in der Lage sind, eine Spirometrie durchzuführen, kann aus Gründen der Kooperationsfähigkeit eine vollständige Bodyplethysmografie in der Regel erst ab dem Schulkindalter erfolgen. Eine polnische Studie zeigte, dass das diagnostische Einbeziehen der Bodyplethysmografie zusätzlich zur Spirometrie im Reversibilitätstest die frühere Diagnose des Asthmas ermöglichte [152]. Zudem bietet die Bodyplethysmografie die Möglichkeit, eine behandlungsbedürftige Überblähung zu erkennen, die sich spirometrisch nicht nachweisen lässt.

\subsubsection{Diffusionskapazität für Kohlenmonoxid}

Die Messung der Diffusionskapazität für Kohlenmonoxid (DLCO) mit dem Einatemzugverfahren eignet sich zur Charakterisierung des intrapulmonalen Gasaustausches. Da dieser in der Regel bei Patienten mit Asthma nicht pathologisch verändert ist, eignet sich die DLCO in besonderer Weise zur differenzialdiagnostischen Abgrenzung des Asthmas von der COPD [153].

\subsubsection{Arterielle Blutgasanalyse}

Die Bestimmung der arteriellen Blutgase ist in der Regel nicht erforderlich und kann meist durch die Bestimmung der Sauerstoffsättigung ersetzt werden. Bei einer schweren Exazerbation $\left(\mathrm{FEV}_{1}<50 \%\right.$ Soll bzw. Peak-flow-Werte $<50 \%$ der Bestwerte, Verschlechterung unter Akuttherapie) ist dagegen die Blutgasanalyse hilfreich, um die Indikation zu einer Sauerstofftherapie zu stellen und um frühzeitig einen Anstieg des $\mathrm{pCO}_{2}$ objektivieren zu können.

\subsubsection{Exspiratorischer Spitzenfluss \\ (Peak expiratory flow, PEF)}

Die Messung des Peak expiratory flow (PEF) wird bei Asthma empfohlen [2]. Die alleinige PEF-Messung ist für die Diagnostik des Asthmas nicht ausreichend. Die häusliche Messung des PEF-
Wertes ist jedoch für die Beurteilung des Verlaufs der Erkrankung und die Beurteilung der Asthmakontrolle hilfreich. Die täglich zweimalige Messung (morgens und abends, vor Inhalation von Medikamenten) erlaubt die Einschätzung der PEF-Variabilität [\%] (Formel: (höchster-niedrigster Wert während einer Woche)/höchster Wert $\times 100$ [\%]. Beispiel: höchster PEF= $400 \mathrm{l} / \mathrm{min}$, niedrigster $\mathrm{PEF}=300 \mathrm{l} / \mathrm{min}$, PEF-Variabilität $=(400-$ $300) / 400 \times 100=25 \%$ ) [154]. Eine PEF Variabilität $\geq 10 \%$ spricht für ein Asthma.

Regelmäßige häusliche Peak-flow-Messungen werden im Kindesalter vor allem im Rahmen von Patientenschulungen eingesetzt. Eine generelle Empfehlung gibt es nicht; in Einzelfällen kann aber der Einsatz gerechtfertigt sein, insbesondere wenn es dem Kind schwer fällt, Atemwegsbeschwerden subjektiv wahrzunehmen. Einschränkend ist hier aber zu erwähnen, dass der PEF meist erst bei einer relativ starken Obstruktion eine Veränderung aufzeigt und dies auch zur falschen Sicherheit führen kann.

\subsection{Nicht invasive Diagnostik der Atemwegs- entzündung}

\subsubsection{Atemkondensat}

Die Bestimmung von Markern im exhalierten Atemkondensat ist bisher für die Diagnostik und Verlaufsbeurteilung des Asthmas nicht validiert.

\subsubsection{Spontanes oder induziertes Sputum}

Die Bestimmung der Gesamtzellzahl und der Zelldifferenzierung des spontanen oder nach Inhalation von hypertoner Kochsalzlösung gewonnenen Sputums sind aufwendig und können daher bisher für den klinischen Alltag nicht empfohlen werden.

In Studien führte die Steuerung der Asthmatherapie auf Basis der Zytologie des induzierten Sputums ( $\geq 3 \%$ eosinophile Granulozyten) bei Patienten mit persistierenden Symptomen und/oder Exazerbationen trotz Therapie mit hochdosierten ICS und LABA zu einer Reduktion der Exazerbationsfrequenz bei geringerem Therapiebedarf [155]. Für Kinder und Jugendliche gibt es keine Daten, die den Nutzen eines Monitorings über induziertes Sputum belegen [103].

\subsubsection{Stickstoffmonoxid (FeNO) in der Ausatemluft}

Bei Asthma bronchiale ist die Fraktion des exhalierten Stickstoffmonoxids (FeNO) ein noninvasiver Biomarker der Atemwegsinflammation. Hohe FeNO-Werte in der Ausatmungsluft wurden bei Patienten mit Asthmasymptomen in Korrelation mit einer Typ-2-Atemwegsentzündung gefunden [156-158]. Diese Information kann therapeutisch genutzt werden, denn Patienten mit Typ 2-dominierter Inflammation sind in der Regel Steroid-responsiv $[100,159]$. Im Umkehrschluss sprechen erhöhte FeNO-Werte unter Therapie mit inhalativen Glucocorticoiden trotz klinischer Stabilität gegen eine Reduktion der ICSDosis.

Die Bestimmung des FeNO kann bei Erwachsenen und bei Kindern hilfreich sein bei der Sicherung der Asthmadiagnose, insbesondere, wenn die Messung beim Patienten ohne regelmäßige ICS-Therapie und im obstruktionsfreien Intervall durch- 
- Tab.3 FeNO-Messungen bei Asthma: Grenzwerte und mögliche Indikationen.

\begin{tabular}{|l|l|l|}
\hline Anlass der FeNO-Messung & FeNO $<\mathbf{2 5} \mathbf{~ p p b}$ (bei Kindern FeNO $<\mathbf{2 0} \mathbf{~ p p b )}$ & FeNO $>\mathbf{5 0} \mathbf{p p b}$ (bei Kindern FeNO $>\mathbf{3 5} \mathbf{p p b})$ \\
\hline Diagnosestellung Asthma & $\begin{array}{l}\text { alternative Diagnosen prüfen } \\
\text { Steroidsensibilität weniger wahrscheinlich }\end{array}$ & $\begin{array}{l}\text { unterstützt die Verdachtsdiagnose } \\
\text { Steroidsensibilität/Typ-2-Entzündung wahrscheinlich }\end{array}$ \\
\hline Symptome unter Therapie & $\begin{array}{l}\text { alternative Diagnosen prüfen } \\
\text { Steroiddosis-Erhöhung weniger sinnvoll }\end{array}$ & $\begin{array}{l}\text { Therapieadhärenz/Allergenexposition prüfen } \\
\text { bei Adhärenz: Steroiddosis-Erhöhung sinnvoll }\end{array}$ \\
\hline Symptomfreiheit unter Therapie & Reduktion der Steroiddosis erwägen & Reduktion der Steroiddosis vermeiden \\
\hline
\end{tabular}

geführt wird [160]. Bei allergischem Asthma wurden höhere FeNO-Werte als bei nicht allergischem Asthma gefunden [161]. Niedrige FeNO-Werte allein erlauben dagegen nicht den differenzialdiagnostischen Ausschluss von Asthma bronchiale [162].

Die FeNO-Messung im Kindes-, Jugend- und Erwachsenenalter ist technisch schnell und einfach durchzuführen und reproduzierbar [156]. Die empfohlenen unteren Grenzwerte der American Thoracic Society (ATS) sind für FeNO $<25$ ppb $(<20$ ppb bei Kindern) [156].

Die FeNO-Messung hat eine Rolle nicht nur in der Diagnostik, sondern auch im Langzeitmanagement [163-165]:

1. Bei Kindern und Jugendlichen erwies sich das regelmäßig kontrollierte FeNO als aussagekräftiger Parameter, um nach geplantem ICS-Absetzen einen Asthmarückfall noch vor dem Auftreten klinischer Symptome vorherzusagen [166, 167].

2. FeNO kann zur Beurteilung der Therapieadhärenz mit herangezogen werden: Erhöhte FeNO-Werte während einer inhalativen Glucocorticosteroid-Therapie können auf schlechte Therapieadhärenz zurückzu führen sein

( Tab. 3) [168].

3. Bei schwangeren Asthmapatientinnen sind FeNO-Messungen sinnvoll, da auf der Basis dieser Messergebnisse die Asthmakontrolle bei gleichzeitiger Reduktion der Dosis der inhalativen Glucocorticosteroide ggf. verbessert werden kann [169].

Zusammenfassend ist die Messung der Atemwegsinflammation mittels FeNO ein wertvoller Baustein im Rahmen von Diagnostik und Management ( $\triangleright$ Tab.3). Welche Patienten von einer FeNO-Messung am meisten profitieren, kann allerdings derzeit nicht abschließend beurteilt werden.

\subsection{Röntgendiagnostik}

Eine Röntgenaufnahme der Thoraxorgane in 2 Ebenen bei Erwachsenen ist bei der Erstdiagnostik bei Verdacht auf Asthma sinnvoll, um andere Erkrankungen auszuschließen. Die Durchführung einer Computertomografie der Thoraxorgane sollte dann erwogen werden, wenn die Differenzialdiagnostik anderer pulmonaler Erkrankungen, die mit Husten, Auswurf und Luftnot einhergehen, klinisch erforderlich ist. Aufgrund der Häufigkeit der Beteiligung der oberen Atemwege, insbesondere bei den nicht allergischen Formen eines Asthmas, sollte bei hinweisenden Beschwerden eine Computertomografie der Nasennebenhöhlen erwogen werden.
Eine postero-anteriore Röntgenaufnahme des Thorax bei Kindern und Jugendlichen kann dann sinnvoll sein, wenn es gilt, differenzialdiagnostisch andere Ursachen der Symptome auszuschließen.

\subsection{Labordiagnostik}

In Hinblick auf personalisierte Asthmatherapien erscheint es sinnvoll, die absolute Eosinophilenzahl im Blut des Asthmapatienten zu bestimmen (Formel: Leukozytenzahl im Blut/ $\mu \mathrm{L}$ : $100 \times$ Eosinophile in \%=Eosinophilenzahl $/ \mu \mathrm{L})$. Eosinophile im Blut weisen die höchste Korrelation mit Eosinophilen im Sputum auf.

Die Eosinophilenkonzentration (Absolutwert/ $\mu \mathrm{L}$ ) im Blut ist ein breit zur Verfügung stehender Biomarker für die Bestimmung des Phänotyps des eosinophilen Asthmas und hat direkte therapeutische Konsequenzen („personalisierte“ Medizin, AntiIL-5 Therapie). Es gibt derzeit allerdings noch keinen anerkannten Grenzwert für die Diagnose eines ,eosinophilen“ Asthmas. Generell gilt: Je höher die Zahl der Eosinophilen/ $\mu \mathrm{L}$ Blut ist, umso besser ist in der Regel die Wirksamkeit der Anti-IL-5-Therapie.

Leukozytenzahl und CRP können bei Verdacht auf infektbedingte Exazerbation gemessen werden. Sie haben einen untergeordneten Stellenwert.

\subsection{Mikrobiologische Sputumdiagnostik}

Eine mikrobiologische Sputumdiagnostik ist bei Asthmaexazerbationen praktisch nie notwendig. Sie ist jedoch hilfreich zur Differenzialdiagnose bei [170]:

- fehlendem Ansprechen auf eine kalkulierte antibiotische Therapie

- wiederholten Infektionen der Atemwege

- Verdacht auf Bronchiektasenerkrankung

- Vorliegen zusätzlicher immunologischer Erkrankungen

\subsection{Allergologische Stufendiagnostik}

Bei jedem neu diagnostizierten Asthma ist eine allergologische Stufendiagnostik erforderlich. Eine allergische Sensibilisierung ist der häufigste Auslöser der Erkrankung. Ein positiver Pricktest macht bei fraglicher Diagnose das Vorliegen eines Asthmas wahrscheinlicher $[171,172]$. Allergologische Stufendiagnostik:

- Allergieanamnese

- Allergie-Hauttestung

- Bestimmung von spezifischem und Gesamt-IgE

- Provokationstestung

- diagnostische Expositionskarenz. 


\subsubsection{Allergieanamnese}

Bei der Eigenanamnese ist die Exposition gegenüber potenziellen, häuslichen und berufsbedingten Allergenen zu erfragen. Weiter soll die Familienanamnese erhoben werden. Die allergologische Anamnese sollte durch geeignete Fragebögen unterstützt werden.

\subsubsection{Allergie-Hauttestungen}

- Der Pricktest ist die primäre allergologische diagnostische Maßnahme. Es wird eine Sensibilisierung gegenüber häufigen Allergenen (v. a. Pflanzenpollen, Hausstaubmilben, Tierhaare und Schimmelpilze) nachgewiesen. Bei anamnestischen Hinweisen können auch Nahrungsmittelallergene (ggf. nativ) getestet werden.

- Intrakutantests (soweit verfügbar) und Reibtests bleiben speziellen Indikationen vorbehalten.

\subsubsection{Bestimmung des spezifischen IgE und Gesamt-IgE im Serum}

Die Bestimmung von spezifischem IgE im Serum stellt in der Regel eine sinnvolle Ergänzung zum Hauttest dar. Insbesondere kommt sie auch bei Patienten zum Einsatz, bei denen eine Hauttestung nicht möglich ist (Ekzem, überempfindliche Haut mit positiver Reaktion auf das Lösungsmittel, fehlende Reaktion der Haut auf Histamin, fehlende Testsubstanz), oder das Ergebnis nicht eindeutig ablesbar ist (z.B. Dermographismus). Die Bestimmung rekombinanter spezifischer Major- und Minorallergene kann helfen, eine primäre Sensibilisierung von einer Kreuzallergie zu unterscheiden $[173,174]$. Die Gesamt-IgEKonzentration im Serum ist ein eigenständiger Asthmaparameter, der mit der Konzentration von spezifischen IgE-Antikörpern oft nicht korreliert [87]. Die Gesamt-IgE-Konzentration im Serum ist für die Planung einer Anti-IgE-Therapie wichtig. Hohe Gesamt-IgE-Spiegel (über $1000 \mathrm{IU} / \mathrm{L}$ ) können auf eine zusätzliche immunologische Erkrankung (z. B. Churg-Strauss-Syndrom, allergische bronchopulmonale Aspergillose) hinweisen.

Für Kinder lässt sich keine allgemeingültige Aussage machen, ob die allergologische Diagnostik per Haut-Prick-Test oder per Bestimmung spezifischer IgE-Antikörper geeigneter zur korrekten Diagnosefindung ist $[175,176]$.

Praktikabilitäts- oder Wirtschaftlichkeitsgründe werden zur primären Wahl der einen oder anderen Methode Anlass geben. Bei Diskrepanz der primären Befunde zur Anamnese sollte auf jeden Fall der jeweils andere Test bzw. eine weiterführende allergologische Diagnostik erfolgen.

\subsubsection{Spezifische nasale und bronchiale Provokation}

In der Regel sind spezifische inhalative Provokationstests entbehrlich. Sie sind jedoch in Einzelfällen (zum Beispiel in der Arbeitsmedizin) hilfreich, sofern Anamnese, Pricktest und das spezifische IgE keine eindeutige Zuordnung erlauben. Bei der Durchführung ist stets die Möglichkeit einer Notfalltherapie vorzuhalten. Die Indikation zu einer spezifischen nasalen Provokation kann vor einer geplanten Immuntherapie gegeben sein.

Bei Kindern sind spezifische bronchiale Provokationstestungen im Einzelfall durchführbar, sollten aber individuell indiziert sein.

\subsubsection{Diagnostische Expositionskarenz}

Die Expositionskarenz kann wichtige Hinweise auf die Bedeutung eines Allergens geben. Dies gilt insbesondere für Patienten, die Tiere im häuslichen Umfeld halten sowie bei der Klärung der Bedeutung beruflicher Allergene.

\subsection{Allgemeine Anmerkungen zur Diagnostik und Differenzialdiagnostik des Asthmas}

Die Diagnose Asthma kann nicht immer bereits bei der Eingangsuntersuchung gestellt werden. Daher sind Verlaufsbeobachtungen sinnvoll, da sie Aufschluss über die spontanen und/ oder medikamentös verursachten Schwankungen des Krankheitsbildes erlauben.

\section{Kriterien und Wahrscheinlichkeit der Diagnose eines Asthmas}

1. Asthma gesichert bei Vorhandensein entsprechender Symptome und Befunde (s.o.) plus

- Nachweis einer Obstruktion $\left(\mathrm{FEV}_{1} / \mathrm{FVC}<\mathrm{LLN}\right)$ und

- Normalisierung der Lungenfunktion (einschließlich $\mathrm{FEV}_{1} / \mathrm{FVC}$ ) im Reversibilitätstest bzw. unter antiasthmatischer Stufentherapie

2. Asthma wahrscheinlich bei Vorhandensein entsprechender Symptome und Befunde (s. o.) plus

- Nachweis einer Obstruktion $\left(\mathrm{FEV}_{1} / \mathrm{FVC}<\mathrm{LLN}\right)$ und Teilreversibilität, zusätzlich

- Therapieansprechen (4 Wochen ICS \pm weitere Antiasthmatika) betreffend sowohl die Lungenfunktion als auch die Symptome oder

- normale oder obstruktive Lungenfunktion mit signifikantem $\mathrm{FEV}_{1}$-Abfall während oder bis zu 30 Minuten nach körperlicher Belastung als indirekte Provokation (Anstrengungskomponente des Asthmas, eventuell auch mit Kaltluftprovokation oder Mannitol), ggf. Zunahme des sRaw um mindestens $100 \%$ und auf $>2 \mathrm{kPa} \times \sec$ [149] oder

- keine oder keine reversible Obstruktion in der Lungenfunktionsprüfung, aber Nachweis einer bronchialen Hyperreagibilität im direkten Provokationstest (z. B. Methacholin) oder von PEF-Variabilität $>10$ \% falls zuverlässige Peak-flow-Messungen vorhanden sind. Hier müssen ggf. der Verlauf und das Ansprechen auf die Therapie in die Beurteilung einbezogen werden oder

- teilreversible Obstruktion in der Lungenfunktionsprüfung ohne Normalisierung nach vier Wochen einer antiasthmatischen Stufentherapie bei gutem klinischen Ansprechen. Hier sollten wiederum Anamnese (Zigarettenraucher?) und klinischer Verlauf in die Beurteilung einbezogen werden.

3. Asthma unwahrscheinlich

- Symptome nicht typisch für Asthma und

- keinerlei Reversibilität der Obstruktion nachweisbar oder

- keine Obstruktion nachweisbar und

- keine bronchiale Hyperreagibilität nachweisbar bei einem symptomatischen Patienten 


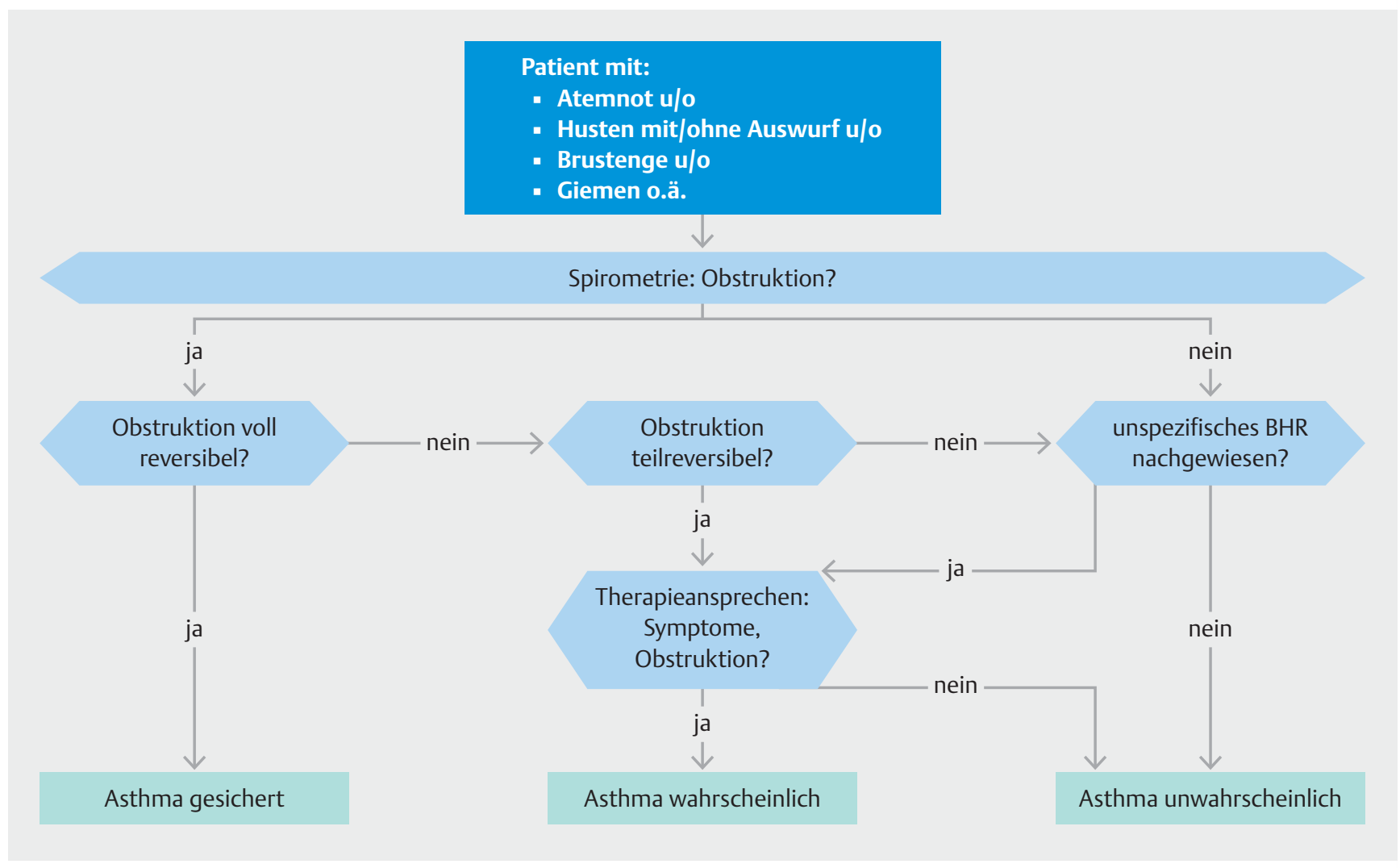

- Abb. 1 Klinischer Algorithmus der Asthmadiagnostik.

Bei Kindern wird bei verdächtiger Anamnese und Klinik die Diagnose Asthma gestellt, wenn sich eine reversible obstruktive Ventilationsstörung dokumentieren lässt (im Reversibilitätstest oder im Provokationstest, siehe oben). Manchmal ist bei normaler Einsekundenkapazität eine isolierte reversible Obstruktion nur anhand von MEF 25-75 oder der Form der Fluss-Volumen-Kurve nachweisbar. Die Diagnose wird gestützt durch einen positiven allergologischen Befund.

Im Kleinkindalter lässt sich die Diagnose in der Regel nicht mit absoluter Sicherheit stellen; wichtige Verdachtsmomente sind Atopie, positive Familienanamnese für Allergien und/oder Asthma und infektunabhängige respiratorische Symptome, wobei es gilt, wichtige Differenzialdiagnosen auszuschließen (z. B. Mukoviszidose, gastroösophagealer Reflux).

Diagnostischer Algorithmus Die Diagnostik des Asthmas ist dadurch erschwert, dass keines der Merkmale - Anamnese, Symptome, klinische und Lungenfunktionsbefunde - für das Asthma pathognomonisch ist. Die Sensitivität und Spezifität der einzelnen Merkmale sind nicht befriedigend. Dies gilt sowohl für Erwachsene als auch für Kinder. Die Diagnose des Asthmas setzt auch eine sorgfältige Differenzialdiagnose voraus (vgl. > Tab. 2 und $>$ Tab. 5 ).
Die häufigste Differenzialdiagnose im Erwachsenenalter ist die COPD. In klassischen Fällen bereitet die Abgrenzung keine Schwierigkeiten. Als Beispiele können der 18-jährige Heuschnupfenpatient mit ausschließlich saisonalem Asthma und normaler Lungenfunktion im Intervall einerseits und der 70 -jährige starke Raucher mit Beschwerdebeginn nach dem 40. Lebensjahr mit persistierender und im Verlauf progressiver Bronchialobstruktion andererseits genannt werden. In vielen Fällen in der täglichen Praxis ist die Abgrenzung jedoch schwierig. Die - Tab. 4 stellt die wichtigsten Merkmale von Asthma und COPD gegenüber. Es gibt, bis auf die volle Reversibilität der obstruktiven Ventilationsstörung, kein einzelnes spezifisches Merkmal für Asthma oder COPD. Die Initialtherapie orientiert sich an der Faustregel, dass erstmalig auftretende obstruktive Atembeschwerden bei Nichtrauchern und unter 40-jährigen Patienten zunächst eher für ein Asthma, bei über 40-jährigen Zigarettenrauchern eher für eine COPD sprechen. Die Diagnose ergibt sich aus der Synthese der Merkmale, besonders im Verlauf durch wiederholte Untersuchungen und unter Berücksichtigung des Ansprechens auf die Therapie [177-179]. Eine normale Diffusionskapazität spricht für Asthma, ebenso eine gute Reversibilität der obstruktiven Ventilationsstörung (>12\% und >400 ml) [180]. Im Einzelfall ist es aber nicht möglich, allein aufgrund der Reversibilität (oder Überempfindlichkeit) Asthma und COPD sicher zu trennen. Es ist zu berücksichtigen, dass auch Mischbilder aus Asthma und COPD vorliegen können [181, 182]. 
- Tab.4 Typische Merkmale von Asthma und COPD bei Erwachsenen.

\begin{tabular}{|l|l|l|}
\hline Merkmal & Asthma & COPD \\
\hline Alter bei Erstdiagnose & häufig: Kindheit, Jugend & meist nicht vor der 6. Lebensdekade \\
\hline Tabakrauchen & $\begin{array}{l}\text { kein direkter Kausalzusammenhang; Verschlechterung } \\
\text { durch Tabakrauchen möglich }\end{array}$ & direkter Kausalzusammenhang \\
\hline Hauptbeschwerden & anfallsartig auftretende Atemnot & Atemnot bei Belastung \\
\hline Verlauf & variabel, episodisch & meist progredient \\
\hline Allergie & häufig & kein direkter Kausalzusammenhang \\
\hline Obstruktion & variabel, reversibel, oft aktuell nicht vorhanden & immer nachweisbar \\
\hline Diffusionskapazität & normal & erniedrigt \\
\hline FeNO & oft erhöht & normal bis niedrig \\
\hline Bluteosinophile & häufig erhöht & meist normal \\
\hline Reversibilität der Obstruktion & diagnostisches Kriterium, oft voll reversibel & nie voll reversibel \\
\hline Überempfindlichkeit der Atemwege & meist vorhanden & selten \\
\hline $\begin{array}{l}\text { Ansprechen der Obstruktion auf } \\
\text { Glucocorticosteroide }\end{array}$ & regelhaft vorhanden & selten \\
\hline
\end{tabular}

- Tab.5 Wichtige Differenzialdiagnosen des Asthmas bei Kindern.

\section{Wichtige Differenzialdiagnosen des Asthmas bei Kindern}

\section{Anamnese}

- Symptome seit der Geburt, peripartal respiratorische Probleme:

- cystische Fibrose (CF), chronische Lungenerkrankung nach Frühgeburtlichkeit/ broncho-pulmonale Dysplasie; primäre ziliäre Dysfunktion (PCD); angeborene Lungenfehlbildung

- Familienanamnese mit pulmonalen Erkrankungen

- CF, neuromuskuläre Erkrankungen, Immundefekt, PCD

- akutes Auftreten ohne vorherige Probleme

- Fremdkörperaspiration

\section{Symptome}

- Fieber, Beschwerden vonseiten der oberen Atemwege

- akuter respiratorischer Infekt (Bronchitis, Bronchiolitis, Bronchopneumonie)

- produktiver Husten

- CF, PCD, Bronchiektasen, protrahierte bakterielle Bronchitis; rezidivierende Aspirationen; Immundefekt

- nächtliche Symptome, verstärkte Spuckneigung

- obere Atemwegsprobleme, gastroösophagealer Reflux mit rezidivierenden Aspirationen

- anfallsartiger Husten

- Pertussis/postinfektöse Hyperreagibilität, Dysphagie, Schluckstörung

- Kurzatmigkeit mit Schwindel, Kribbelparaesthesien

- dysfunktionelle Atmung, z. B. Hyperventilation

- in- und/oder exspiratorischer Stridor

- angeborene Fehlbildung (Stenose oder Malazie im Bereich der großen Atemwege); Laryngitis, Tracheitis

- abnorme Stimme, Heiserkeit

- laryngeales Problem

- lokalisierte thorakale Befunde

- angeborene Fehlbildung, postinfektiöse Veränderungen, Tuberkulose

- Trommelschlegelfinger

- CF, Bronchiektasen

- Gedeihstörung

- CF, Immundefekt, gastroösophagealer Reflux, interstitielle Lungenerkrankung

\section{Untersuchungsbefunde}

- lokalisierte radiologische Veränderungen

- angeborene Fehlbildung, CF, postinfektiöse Veränderungen

- Fremdkörperaspiration, rezidivierende Aspirationen bei Schluckstörung oder gastroösophagealem Reflux, Bronchiektasen, Tuberkulose 
Die globalen Initiativen für Asthma und COPD haben für Patienten mit einer obstruktiven Atemwegserkrankung, die nicht sicher einer der beiden Entitäten zuzuordnen ist, die initiale Diagnose eines ACO (Asthma-COPD-Overlap) vorgeschlagen [183]. Bei Patienten, die diagnostische Kriterien für Asthma und COPD aufweisen, sollten beide Diagnosen gestellt (und nach ICD kodiert) werden. Als typisches Beispiel hierfür gilt der allergische Asthmapatient mit Atembeschwerden seit der Kindheit, der Jahrzehnte lang geraucht und eine nicht mehr reversible Bronchialobstruktion und Emphysem entwickelt hat. Zur Differenzialdiagnostik müssen ggf. weiterführende Untersuchungsmethoden zur Anwendung kommen.

\section{Medikamentöse Therapie des Asthmas}

Die Empfehlungen zur medikamentösen Therapie basieren auf dem Verständnis der Pathologie und Pathophysiologie des Asthmas und auf der Extrapolation kontrollierter klinischer Prüfungen, die die Wirksamkeit antiasthmatischer Medikamente auf Parameter der Erkrankungskontrolle (z. B. Asthma-Symptome, Lungenfunktion oder Bedarf an Bronchodilatatoren zur Symptomkontrolle) untersucht haben [2,70,184-186]. Die Empfehlungen beziehen sich auf Kinder ab dem 6. Geburtstag sowie Jugendliche und Erwachsene. Klein- und Vorschulkinder sind als eigene Patientengruppe zu betrachten und in dieser Darstellung ausgenommen.

Die Empfehlungen zu einer medikamentösen Therapie müssen aktuelle Beschwerden, die Ergebnisse klinischer Untersuchungen und Funktionsdiagnostik, den aktuellen Asthmaschweregrad, die aktuelle Behandlung einschließlich der Bewertung der Therapieadhärenz, die pharmakologischen Eigenschaften und individuellen Verträglichkeiten der antiasthmatischen Medikamente, pharmakoökonomische Gesichtspunkte sowie die Komorbidität(en) des Patienten berücksichtigen. Ziel der medikamentösen Therapie ist das Erreichen von Asthmakontrolle durch Suppression der asthmatischen Entzündung mit Verminderung der bronchialen Hyperreagibilität und Reduktion der (variablen) Atemwegsobstruktion [187-189]. Be- handlungsempfehlungen müssen sowohl die prinzipielle Heterogenität des Asthmas als auch die variable Ausprägung der Erkrankung bei verschiedenen Patienten und die individuelle Variabilität der Erkrankung im Verlauf in Betracht ziehen. Wesentliche Elemente jeder Langzeittherapie sind daher die Verlaufsbeurteilung der Behandlung, die insbesondere die Beeinflussung der Symptome und die Ergebnisse der Lungenfunktionsprüfungen einbezieht, und die entsprechende Anpassung der Behandlung im Verlauf der Erkrankung. Oftmals stellen Behandlungspläne einen Kompromiss zwischen den Therapieempfehlungen des Arztes und der Bereitschaft zur Therapieadhärenz des Patienten dar. Ziel ist, eine bestmögliche Asthmakontrolle mit so wenigen Medikamenten als möglich in jeweils optimaler Dosierung und mit so wenigen Nebenwirkungen als möglich zu erreichen und dauerhaft zu erhalten.

Die Medikamente werden in Langzeittherapeutika (sogenannte „Controller“ oder Dauermedikamente zur Langzeitkontrolle) $[188,190$ - 192] und in Bedarfsmedikamente (sogenannte „Reliever“) $[193,194]$ unterteilt. Asthmatherapeutika können grundsätzlich inhalativ, oral und/oder parenteral (subkutan, intramuskulär und intravenös) verabreicht werden. Die Möglichkeit der Applikation der meisten Medikamente durch Inhalation ist eine Besonderheit obstruktiver Atemwegserkrankungen. Dadurch können mit geringeren Wirkstoffmengen höhere topische Konzentrationen $[195,196]$ bei oft rascherem Wirkeintritt und geringeren systemischen (Neben-)Wirkungen erzielt werden. Oral applizierte Medikamente sind nur indiziert bei unzureichender Wirkung inhalativer Präparate bzw. dann, wenn einem Patienten eine effektive Inhalation trotz Schulung nicht möglich ist.

Medikamentöse Langzeittherapie Die medikamentöse Langzeittherapie des Asthmas wird üblicherweise nach einem Stufenplan durchgeführt. Die Zahl der eingesetzten Medikamente sowie deren Dosierung und Applikationshäufigkeit orientiert sich am Grad der Asthmakontrolle und am Schweregrad der Erkrankung. Die Einschätzung der Asthmakontrolle basiert auf den Beschwerden des Patienten (

- Tab. 6 Grad der Asthmakontrolle. Die oberen 4 Kriterien (Symptome tagsüber und nachts, Bedarfsmedikation, Aktivitätseinschränkung) entsprechen dem vereinfachten Schema zur Messung der Asthmakontrolle gemäß GINA [2]. Die unteren beiden Kriterien (FEV1, Exazerbation; dick umrandet) sind Zusatzkriterien zur erweiterten Prüfung der Asthmakontrolle.

\begin{tabular}{|l|l|l|l|}
\hline & $\begin{array}{l}\text { kontrolliertes Asthma } \\
\text { bei Kindern }\end{array}$ & $\begin{array}{l}\text { kontrolliertes Asthma } \\
\text { bei Erwachsenen }\end{array}$ & $\begin{array}{l}\text { teilweise kontrolliertes } \\
\text { Asthma }\end{array}$ \\
\hline Symptome tagsüber & keine & $\mathbf{1 - 2}$ Kriterien erfüllt \\
\hline Symptome nachts & keine & keine & jedes Symptom \\
\hline Bedarfsmedikation & keine & $\leq 2 \times /$ Woche & $>2 \times /$ Woche \\
\hline Aktivitätseinschränkung & keine & keine & jede Einschränkung \\
\hline FEV 1 & normal & normal & vermindert \\
\hline Exazerbation & keine & keine & mindestens $1 \times /$ Jahr \\
\hline
\end{tabular}




\section{EMPFEHLUNGEN}

E9 Treten anstrengungsinduzierte Asthmasymptome bei adäquat behandelten Patienten auf, sollen kurzwirksame inhalative Beta-2-Sympathomimetika (SABA) vor körperlicher Belastung eingesetzt werden.

E10 Speziell für die Kinder: Sind regelmäßig kurzwirksame Beta-2-Sympathomimetika vor Sport notwendig, soll die antiinflammatorische Langzeittherapie angepasst werden.

E11 Zur Prophylaxe von anstrengungsinduzierten Asthmasymptomen kann alternativ neben inhalativen Beta-2Sympathomimetika ein Leukotrien-Rezeptor-Antagonist in der Dauertherapie eingesetzt werden.

E12 Bei Vorliegen mehrerer Darreichungsformen antiasthmatischer Wirkstoffe sollen andere Formen als die inhalative Therapie nur in begründeten Ausnahmefällen verwendet werden.

E13 In jeder Therapiestufe soll zur Symptomkontrolle ein kurzwirksames Beta-2-Sympathomimetikum eingesetzt werden. Ab Therapiestufe 3 kann bei Jugendlichen und Erwachsenen alternativ eine Fixkombination aus ICS und Formoterol im Rahmen eines (S)MART-Konzeptes zur Symptomkontrolle eingesetzt werden.

E14 Bei unbehandelten Patienten sollte eine Asthmatherapie wie folgt initiiert werden:

- bei teilweise kontrolliertem Asthma mit einem niedrig dosierten ICS (Therapiestufe 2)

- bei unkontrolliertem Asthma mindestens mit einer Therapie der Stufe 3

E15 Ab Therapiestufe 2 soll stets eine regelmäßige Langzeittherapie mit inhalativen Glucocorticoiden (ICS) erfolgen, aufgeteilt in 2 tägliche Dosen oder als Einmaldosis.

E16 Speziell für Kinder: Bei Stufe 2 ist eine ICS-Therapie 1. Wahl; alternativ kann Montelukast zum Einsatz kommen. E17 In Stufe 3 soll bei erwachsenen Asthmapatienten bevorzugt eine freie oder fixe Kombination aus einem niedrigdosierten ICS und einem inhalativen langwirkenden Beta-2Sympathomimetikum (LABA; Formoterol, Salmeterol) oder alternativ ein ICS in mittlerer Dosis eingesetzt werden.

E18 Speziell für Kinder: In Stufe 3 soll ICS als Monotherapie in mittlerer Dosis eingesetzt werden.

E19 Eine Therapie mit einem LABA soll nur in Kombination mit einem ICS erfolgen, nicht als Monotherapie.

E20 Alternativ zum LABA ab Stufe 3 sollten LTRA eingesetzt werden; als weitere Alternative können in begründeten Fällen bei Erwachsenen Theophyllin-Präparate mit verzögerter Wirkstoff-Freisetzung verordnet werden. Bei Kin- dern und Jugendlichen sollen Theophyllin-Präparate in Stufe 3 nicht eingesetzt werden

E21 In Therapiestufe 4 soll ICS in mittlerer bis hoher Dosis in freier oder fixer Kombination mit einem LABA gegeben werden, ggf. ergänzt mit Tiotropium. Ein Theophyllin-Präparat mit verzögerter Wirkstoff-Freisetzung kann in begründeten Fällen alternativ oder additiv gegeben werden.

E22 Speziell für Kinder und Jugendliche: In Therapiestufe 4 soll ICS in mittlerer bis hoher Dosis in fixer Kombination mit einem LABA und/oder in Kombination mit einem LTRA eingesetzt werden (allerdings ist Montelukast nur für leichtes bis mittelschweres Asthma in Deutschland zugelassen). Nur ausnahmsweise sollte Theophyllin als Add-on-Therapie erwogen werden.

E23 Additiv zu den Medikamenten der Therapiestufe 4 sollen in der Therapiestufe 5 Tiotropium und/oder Biologika (Anti-IgE oder Anti-IL5) bei partiell kontrolliertem oder unkontrolliertem Asthma eingesetzt werden, sofern die Indikation für diese Präparate erfüllt wird. Die Langzeittherapie mit systemischen Glucocorticosteroiden soll wegen der Gefahr schwerer Nebenwirkungen intermittierend oder dauerhaft in der niedrigsten noch effektiven Dosis nur dann empfohlen werden, wenn Biologika nicht indiziert sind, nicht ausreichend wirken bzw. wenn trotz des kombinierten Einsatzes der verschiedenen Therapieoptionen in Stufe 4 und 5 das Asthma unkontrolliert ist.

E24 Speziell für Kinder und Jugendliche: Vor Übergang auf Therapiestufe 4 soll eine diagnostische Reevaluation erfolgen.

E25 Der Grad der Asthmakontrolle soll regelmäßig überprüft werden, um festzustellen, ob eine Therapieänderung notwendig ist. Ineffektive Therapien sollen abgesetzt werden. Ziel ist die Erhaltung der Asthmakontrolle mit der geringstmöglichen Zahl antiasthmatischer Wirkstoffe in niedrigstmöglicher Dosis.

E26 Die Reduktion der medikamentösen ICS/LABA-Therapie sollte, wenn möglich, mit der Halbierung der ICS-Dosis begonnen werden. Ist eine niedrige tägliche ICS-Dosis erreicht, sollte die Beendigung der Therapie mit additiv verabreichten Medikamenten erwogen werden. Bei Reduktion der Therapie soll dem Stufenplan umgekehrt gefolgt werden.

E27 Eine Deeskalation der Asthmatherapie erfordert eine engmaschige Überwachung des Patienten. Vor einer Reduktion der Therapie soll das Asthma mindestens drei Monate kontrolliert sein. nach GINA [2]). Der Schweregrad der Erkrankung richtet sich nach dem Therapie-Ansprechen und kann daher typischerweise nicht bei Erstdiagnose bestimmt werden ( $\triangleright$ Tab. 7: Asthmaschweregrade bei Erwachsenen). Der Asthmaschweregrad basiert auf der Therapiestufe, die zur Erhaltung der Symptomkon- trolle und Prävention von Exazerbationen erforderlich ist. Insofern ist der Asthmaschweregrad keine statische, sondern eine variable Einschätzung, die sich im Erkrankungsverlauf ändern kann. 
- Tab.7 Asthmaschweregrad-Einteilung bei erwachsenen Patienten.

\begin{tabular}{|l|l|}
\hline Asthmaschweregrad & Charakteristika \\
\hline leicht & $\begin{array}{l}\text { gute Asthmakontrolle unter Medikation } \\
\text { der Therapiestufe 1 oder } 2 \text { erreichbar }\end{array}$ \\
\hline mittelgradig & $\begin{array}{l}\text { gute Asthmakontrolle unter Medikation } \\
\text { der Therapiestufe } 3 \text { oder 4 erreichbar }\end{array}$ \\
\hline schwer & $\begin{array}{l}\text { nicht gut kontrolliertes Asthma unter } \\
\text { hochdosierter ICS-LABA-Therapie } \\
\text { oder Verlust der Asthmakontrolle } \\
\text { bei Reduktion dieser hochdosierten } \\
\text { ICS-LABA-Therapie; Notwendigkeit } \\
\text { der Therapiestufe 5 }\end{array}$ \\
\hline
\end{tabular}

Das Konzept einer Stufentherapie (s. $>$ Abb. 2 und $>$ Tab. 8) beinhaltet, dass die Therapie stufenweise intensiviert wird, falls sich mit dem gegenwärtigen Asthmamanagement keine ausreichende Kontrolle erzielen lässt, eine adäquate Therapietreue des Patienten vorausgesetzt. Häufige (z. B. bei Erwachsenen $>2 \times$ pro Woche) Symptome tagsüber (z. B. Husten, Atemnebengeräusche, Atemnot), nächtliche Beschwerden aufgrund des Asthmas, ein vermehrter (z. B. bei Erwachsenen $>2 \times$ pro Tag) Bedarf an raschwirkenden Bronchodilatatoren wegen Symptomen und/oder Einschränkungen bei Alltagsaktivitäten aufgrund des Asthmas zeigen eine inadäquate Asthmakontrolle und die Notwendigkeit zur Steigerung der Intensität der Behandlung an. Messungen des exspiratorischen Spitzenflusses (Peak flow) und der Peak-flow-Variabilität sind sowohl zur initialen Einschätzung der Atemflusslimitierung als auch zur Verlaufsbeurteilung und zur engmaschigen Überwachung bei Änderungen der Therapie ergänzend geeignet.

Zur initialen Therapie eines neu diagnostizierten Asthmas bei Erwachsenen, bei dem der tatsächliche Schweregrad der Erkrankung noch nicht bekannt ist, gibt es zwei Konzepte:

- „Step down“-Therapie:

Die initiale Therapie orientiert sich an einem höheren als dem wahrscheinlichen Schweregrad, um eine möglichst rasche Asthmakontrolle zu erzielen. Nach Besserung der Beschwerden bzw. nach Erreichen einer guten Asthmakontrolle wird die Intensität der Medikation für die Langzeittherapie an den tatsächlichen Schweregrad der Erkrankung angepasst.

- „Step up“-Therapie:

Die Behandlung wird mit einer dem wahrscheinlichen Schweregrad entsprechenden Medikation begonnen und im Verlauf dem tatsächlichen Bedarf graduell angepasst. Langfristig sind die klinischen Ergebnisse beider Strategien bei Erwachsenen vergleichbar [197].

Initiale Therapie für Kinder und Jugendliche:

- Für Kinder und Jugendliche sollte abhängig von der initialen Präsentation eine niedrige oder mittelhochdosierte inhalative glucocorticosteroide Therapie begonnen werden. Nur ausnahmsweise ist in der Erstbehandlung eine hochdosierte oder kombinierte Therapie indiziert. Bei über einen längeren
Zeitraum stabiler Erkrankung und guter Asthmakontrolle kann die Therapie stufenweise reduziert werden (z. B. nach einer Behandlungsdauer von 1 - 2 Monaten, bei Therapie mit inhalativen Glucocorticosteroiden frühestens nach 3 Monaten), falls dann keine Verschlechterung der Asthmakontrolle eintritt. In diesem Fall kann bei Kindern und Jugendlichen, die gut kontrolliert unter einer kombinierten Therapie mit ICS und LABA sind, vorübergehend eine Kombinationstherapie aus niedrigdosiertem ICS und LABA verwendet werden. Initial sollte eine niedrigdosierte ICS-Therapie versucht werden (pädiatrische Dosisangaben). Bei unzureichender Asthmakontrolle sollte die ICS-Dosis zunächst in den oberen Niedrigdosisbereich bzw. den untersten mittelhohen Dosisbereich (pädiatrische Dosisangaben) erhöht werden (Stufe 3). Als nächster Schritt ist die Kombination mit einem LABA sinnvoll. Wenn es unter einem LABA nicht zu einer Verbesserung der Symptome kommt, sollte die LABA-Therapie wieder beendet und stattdessen die ICS-Dosis verdoppelt werden. Alternativ kann die Kombination ICS/LTRA versucht werden. Wenn es zu einer Verbesserung der Asthmakontrolle unter ICS/LABA-Therapie kommt, sollte die Kombination fortgesetzt werden.

- Auch bei Erwachsenen lässt sich durch die stufenweise Reduktion der geringste, zur langfristig guten Kontrolle der Erkrankung erforderliche Therapiebedarf festlegen. Wichtig ist, dass Asthmakontrolle, Asthmaschweregrad und Therapiestufe nicht übereinstimmen müssen. So kann beispielsweise bei einem Patienten mit schwerem Asthma und guter Asthmakontrolle unter intensiver Therapie scheinbar ein niedriger Asthmaschweregrad vorliegen. Falls keine ausreichende Krankheitskontrolle mit der Initialtherapie (z. B. innerhalb eines Zeitraumes von einem Monat) erzielt wird, sollten die Behandlung und die Therapieadhärenz überprüft und immer auch die Diagnose überdacht, ggf. die Diagnostik wiederholt bzw. erweitert werden (vgl. Kapitel Diagnostik).

Es ist zu berücksichtigen, dass trotz optimaler Therapie und Therapieadhärenz nicht bei allen Asthmatikern eine gute Asthmakontrolle erreicht werden kann.

Bei der Auswahl ist insbesondere für Kinder und Jugendliche zu beachten, dass die verfügbaren ICS ein unterschiedliches Risiko unerwünschter Wirkungen haben [198]. Es soll die jeweils niedrigste Dosis der Substanz mit dem geringsten Risiko für systemische Wirkungen bevorzugt werden. Auch bei Inhalation im niedrigen Dosisbereich ist eine systemische Wirkung nicht auszuschließen.

Jeder Patient soll von seinem behandelnden Arzt einen aktuellen schriftlichen Therapieplan erhalten. Dieser enthält konkrete Angaben zu Art, Zahl und Dosierung (inklusive Einnahmehäufigkeit) der Medikamente sowie Hinweise zur Therapieintensivierung bei Verschlechterung der Asthmakontrolle bzw. zum Verhalten bei Exazerbationen (Notfallplan). 
D Tab. 8 Tagesdosen inhalativer Glucocorticosteroide $(\mu \mathrm{g})$ für Asthmapatienten gemäß der Einteilung der GINA [2].

\begin{tabular}{|c|c|c|c|c|c|c|}
\hline \multirow[t]{2}{*}{ Wirkstoff } & \multicolumn{2}{|l|}{ niedrige Dosis } & \multicolumn{2}{|l|}{ mittlere Dosis } & \multicolumn{2}{|l|}{ hohe Dosis } \\
\hline & Erwachsene & $\begin{array}{l}\text { Kinder \& Jugend- } \\
\text { liche }\end{array}$ & Erwachsene & $\begin{array}{l}\text { Kinder \& Jugend- } \\
\text { liche }\end{array}$ & Erwachsene & $\begin{array}{l}\text { Kinder \& Jugend- } \\
\text { liche }\end{array}$ \\
\hline Beclometason $^{1}$ & $200-500$ & $100-200$ & $500-1000$ & $200-400$ & $>1000$ & $>400$ \\
\hline Beclometason ${ }^{2}$ & $100-200$ & $50-100$ & $200-400$ & $100-200$ & $>400$ & $>200$ \\
\hline Budesonid, DPI & $200-400$ & $100-200$ & $400-800$ & $200-400$ & $>800$ & $>400$ \\
\hline Ciclesonid, HFA ${ }^{3}$ & 80 & 80 & $160-320$ & $80-160$ & $>320$ & $>160$ \\
\hline $\begin{array}{l}\text { Fluticasonpropionat, } \\
\text { DPI \& HFA }\end{array}$ & $100-250$ & $50-200$ & $250-500$ & $200-400$ & $>500$ & $>400$ \\
\hline Fluticasonfuroat, $\mathrm{DPI}^{3}$ & 100 & 100 & - & - & 200 & 200 \\
\hline Mometason $^{3}$ & $110-220$ & 110 & $220-440$ & $220-440$ & $>440$ & 440 \\
\hline \multicolumn{7}{|c|}{$\begin{array}{l}\text { HFA = Treibgas Hydrofluoralkane; DPI = Trockenpulverinhalator } \\
1 \text { Standard-Partikelspektrum } \\
2 \text { Produkte mit extrafeinem Partikelspektrum } \\
3 \text { keine Zulassung für Kinder < } 12 \text { Jahre. }\end{array}$} \\
\hline
\end{tabular}

- Tab. 9 ICS-Tages-Gesamtdosen bei erwachsenen Patienten, die gemäß Konsensus der ERS und ATS [128] als Hochdosis-ICS-Therapie bei der Definition von schwerem Asthma gelten. Die auf den Inhalatoren angegebenen Dosierungen können von den Dosierungen, die in die Dosisberechnung eingehen, abweichen. Beispiel 1: Bei der Fixkombination Budesonid/Formoterol ist die Inhalationsdosis angegeben, die das Mundstück verlässt (in der höchsten Dosierung: $360 \mu \mathrm{g}$ ), in die Dosisberechnung geht die im Gerät abgemessene Dosis ein (400 $\mu \mathrm{g}$ Budesonid). Beispiel 2: Bei der Fixkombination (Dosieraerosol) Beclometason/Formoterol wird eine Dosis von $100 \mu \mathrm{g}$ Beclometason angegeben, aufgrund der extrafeinen Zubereitung entspricht diese Dosis aber $250 \mu \mathrm{g}$ Beclometason in einer nicht extrafeinen Zubereitung. Das Äquivalent der nicht extrafeinen Dosierung $(250 \mu \mathrm{g})$ geht in die Dosisberechnung ein.

\begin{tabular}{|c|c|}
\hline Beclometason (DPI) & $\geq 2000 \mu \mathrm{g}$ \\
\hline Beclometason (HFA-DA) & $\geq 1000 \mu \mathrm{g}$ \\
\hline Budesonid & $\geq 1600 \mu \mathrm{g}$ \\
\hline Ciclesonid & $\geq 320 \mu \mathrm{g}$ \\
\hline Fluticasonpropionat & $\geq 1000 \mu \mathrm{g}$ \\
\hline Mometasonfuroat & $\geq 800 \mu \mathrm{g}$ \\
\hline
\end{tabular}

\subsection{Medikamentöse Therapie erwachsener Asthmapatienten}

\subsubsection{Anstrengungsinduziertes Asthma}

Bei den meisten Patienten ist ein anstrengungsinduziertes Asthma Ausdruck einer inadäquaten Asthmakontrolle. Deshalb sollte zunächst die medikamentöse Therapie überprüft werden (s. o.). Die Therapieentscheidung bei einer Anstrengungskomponente des Asthmas sollte stets berücksichtigen, ob lediglich eine Prophylaxe bei bevorstehender Anstrengung oder auch eine Dauertherapie wegen eines anstrengungsinduzierten Asthmas infolge Hyperreagibilität bei chronischem Asthma erforderlich ist, sowie ob es sich um ein unbehandeltes Asthma oder ein Anstrengungsasthma als einzige Asthmamanifestation bzw. um ein behandeltes und gut eingestelltes Asthma mit Beschwerden bei körperlicher Belastung handelt. Asthmabeschwerden bei körperlicher Anstrengung können auch Ausdruck von Adipositas, mangelnder körperlicher Fitness oder Asthma-unabhängiger Komorbiditäten sein.

Tab.10 Anstrengungsasthma als einzige Asthmamanifestation.

\begin{tabular}{|l|l|l|}
\hline & SABA & LTRA \\
\hline Prophylaxe & ja & ja \\
\hline Akuttherapie & ja & nein \\
\hline
\end{tabular}




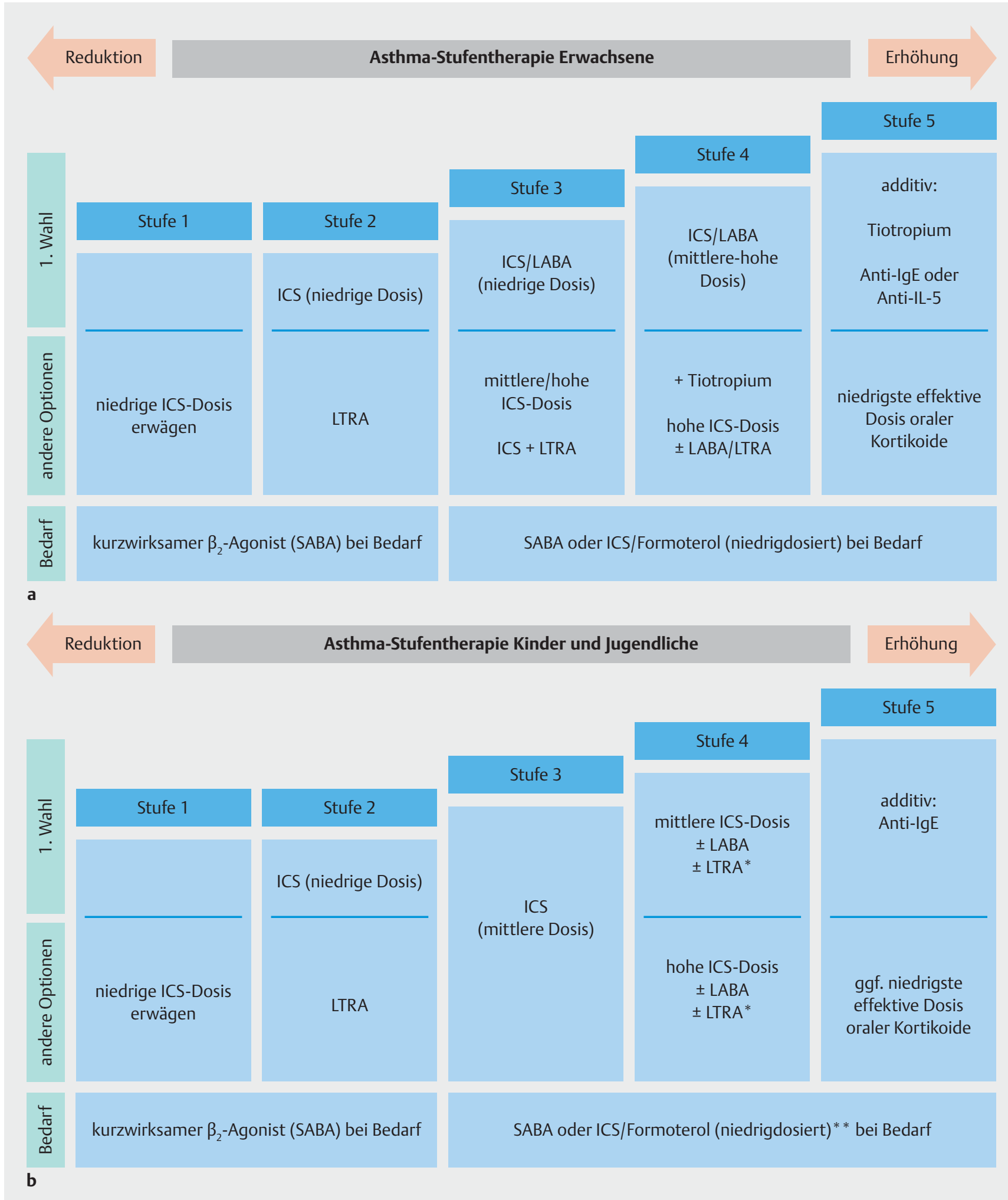

- Abb. 2 a Asthma-Stufentherapie für Erwachsene. b Asthma-Stufentherapie für Kinder und Jugendliche. ICS=inhalatives Kortikosteroid, SABA = kurzwirksames Beta-2-Sympathomimetikum, LABA = langwirksames Beta-2-Sympathomimetikum, LTRA=Leukotrienrezeptor-Antagonist. ${ }^{*}$ Montelukast ist nur für leichtes bis mittelschweres Asthma in Deutschland zugelassen. ${ }^{*}$ bei Jugendlichen $>12$ Jahre. 
\ Tab.11 Behandeltes Asthma mit Beschwerden bei körperlicher Belastung (Anstrengungsasthma).

\begin{tabular}{|l|l|l|l|l|l|}
\hline & ICS & SABA & LABA & ICS/LABA & LTRA \\
\hline Prophylaxe & ja, Dosis erhöhen & ja & ja, falls nicht im Therapieplan & ja, falls nicht im Therapieplan & ja, falls nicht im Therapieplan \\
\hline Akuttherapie & nein & ja & nein & nein & nein \\
\hline
\end{tabular}

Tritt anstrengungsinduziertes Asthma bei Patienten auf, die bereits mit inhalativen Glucocorticosteroiden behandelt werden, sind zusätzlich inhalative raschwirksame Beta-2-Sympathomimetika unmittelbar vor körperlicher Belastung Mittel der Wahl [199, 200]. Abhängig von der Frequenz der Beschwerden (Asthmakontrolle) kann ein Step-up der Langzeittherapie nach Stufenschema sinnvoll sein ( $\triangleright$ Tab. 11).

Zur Prophylaxe von durch körperliche Anstrengung induzierten Asthmasymptomen kann neben den genannten Präparaten auch der Leukotrien-Rezeptor-Antagonist Montelukast in der Dauertherapie wirksam sein und ist in dieser Indikation als Monotherapie auch für Erwachsene zugelassen ( $\$$ Tab. 10). Diese Empfehlungen gelten in gleicher Weise für die Prophylaxe seltener, vorhersehbarer Allergenexpositionen.

Schließlich kann eine kontrollierte Aufwärmphase am Beginn einer körperlichen Anstrengung die Inzidenz und den Schweregrad der Asthmasymptome reduzieren [200, 201].

Treten Asthmasymptome auch unabhängig von körperlicher Anstrengung auf, sollte eine regelmäßige Erhaltungstherapie begonnen bzw. intensiviert werden. In gleicher Weise können Beschwerden bei sonst adäquat behandelten und kontrollierten Patienten durch SABA-Inhalation abgemildert werden ( $\triangleright$ Tab. 11).

Bei Leistungssportlern ist zu berücksichtigen, dass einige zur Prophylaxe und Therapie des Anstrengungsasthmas empfohlene Medikamente unter einem Dopingvorbehalt stehen. Zur aktuellen Information wird auf die nationalen Anti-Doping-Agenturen Deutschlands [118], Österreichs [119] bzw. der Schweiz [120] verwiesen.

\subsubsection{Therapiestufe 1: Kurzwirksames Bedarfs- therapeutikum}

Als bevorzugte Therapieoption soll Patienten in Therapiestufe 1 die bedarfsweise Inhalation eines kurzwirksamen inhalativen Beta-2-Sympathomimetikums (SABA) empfohlen werden [202]. Diese Medikamente sind sehr effektiv zur raschen Linderung von Asthmabeschwerden. Die Evidenz im Hinblick auf die langfristige Effektivität und Verträglichkeit einer Asthmatherapie nur mit einem SABA ist ungenügend, sodass diese Option Patienten mit nur gelegentlichen (z. B. seltener als $2 \times$ pro Monat) oder kurzdauernden (z.B. wenige Stunden) Asthmabeschwerden ohne nächtliches Erwachen wegen Asthma und mit normaler Lungenfunktion vorbehalten bleiben sollte.

Häufigere Symptome oder zusätzliche Risikofaktoren einer künftigen Exazerbation (z. B. FEV $\mathrm{F}_{1}<\mathrm{LLN}$ ) oder eine Exazerbation im zurückliegenden Jahr stützen die Indikation einer regelmäßigen Erhaltungstherapie [203-205]. Diesen Patienten sollte zusätzlich zu einem SABA bei Bedarf eine regelmäßige Erhaltungstherapie mit niedrigen Dosen inhalativer Glucocorticoste- roide empfohlen werden $[203,204]$, umso mehr als die positiven klinischen Effekte von ICS bei leichtgradigem Asthma unabhängig von der Symptomhäufigkeit sind [206].

In begründeten Fällen potenziell ebenfalls zur Symptomkontrolle einsetzbar sind inhalative, rasch wirksame Anticholinergika (Ipratropium), orale Beta-2-Sympathomimetika oder rasch wirksame Theophyllin-Präparate. Allerdings weisen diese Präparate einen deutlich langsameren Wirkeintritt und/oder ein höheres Nebenwirkungsrisiko auf [207].

Wird eine derartige Bedarfsmedikation häufiger als zweimal pro Woche während eines dreimonatigen Zeitraums benötigt, sollte der Patient nach den Empfehlungen für die Therapiestufe 2 behandelt werden. Dies gilt auch, falls die Lungenfunktion zwischen Exazerbationen dauerhaft pathologisch ist. Auch bei in Therapiestufe 1 behandelten Patienten können Asthmaexazerbationen auftreten und müssen konsequent behandelt werden, ggf. mit oralen Glucocorticosteroiden. In diesem Fall ist eine medikamentöse antiinflammatorische Dauertherapie erforderlich.

Das Beta-2-Sympathomimetikum Formoterol weist einen raschen Wirkeintritt auf und ist zur Bedarfsmedikation bei Erwachsenen so effektiv wie ein SABA. Dennoch soll Formoterol als Monotherapie ohne begleitende regelmäßige ICS-Therapie angesichts des Risikos von Exazerbationen nicht zur Bedarfstherapie eingesetzt werden [208].

\subsubsection{Therapiestufe 2: Langzeittherapeutikum plus kurzwirksames Bedarfstherapeutikum}

Als bevorzugte Therapieoption soll Patienten in Therapiestufe 2 eine regelmäßige Therapie mit einem inhalativen Glucocorticosteroid in niedriger Dosis und die bedarfsweise Anwendung eines kurzwirksamen inhalativen Beta-2-Sympathomimetikums (SABA) empfohlen werden.

Patienten mit häufigen Asthmasymptomen ( $\geq 2 /$ Woche; bei Kindern und Jugendlichen jegliche wiederkehrende Beschwerden) trotz Therapie mit einem raschwirksamen Bronchodilatator bei Bedarf benötigen eine entzündungshemmende Dauertherapie, um die Erkrankung akut zu kontrollieren und die Asthmakontrolle langfristig aufrechtzuerhalten [187, 188, 194, 209 -212]. Diesen Patienten soll daher eine regelmäßige, tägliche Behandlung mit einem entzündungshemmenden Wirkstoff empfohlen werden. Mittel der ersten Wahl sind inhalative Glucocorticosteroide in niedriger Dosis [204,213], aufgeteilt in zwei tägliche Dosen oder als Einmaldosis [190] ( Tab. 8). Inhalative Glucocorticosteroide bessern die entzündlichen Schleimhautveränderungen in den Atemwegen und die bronchiale Hyperreagibilität [187, 191 - 194, 214]. Klinisch kommt es zu einer Verbesserung der Lungenfunktion, einer Abnahme der bronchialen Hyperreagibilität [215], der Frequenz von Asthma- 
symptomen, der Frequenz und des Schweregrades von Exazerbationen, Asthma-bedingter Hospitalisierungen und von Todesfällen sowie zu einer Verbesserung der Asthma-bezogenen Lebensqualität [188, 190 - 192, 203, 204, 216, 217].

Die Verwendung einer Inhalationshilfe (Spacer) zur Inhalation des Glucocorticosteroids als Dosieraerosol kann zur Verbesserung der pulmonalen Deposition [218,219] sowie zur Vorbeugung oropharyngealer Nebenwirkungen [220,221] bzw. erhöhter systemischer Absorptionen empfohlen werden [222].

Eine bei den meisten Patienten weniger effektive Alternative in dieser Indikation ist der Leukotrienrezeptor-Antagonist Montelukast [223, 224], der bei Patienten eingesetzt werden kann, die eine ICS-Therapie ablehnen, denen eine Therapie mit ICS nicht möglich ist, die über ausgeprägte lokale Nebenwirkungen inhalativer Glucocorticosteroide klagen oder die eine komorbide allergische Rhinitis aufweisen [225, 226]. Montelukast ist in dieser Indikation als Monotherapie in Deutschland nur für Kinder zugelassen, in anderen Ländern auch für Erwachsene [227-233].

Eine weitere Alternative bei bislang nicht mit einer regelmäBigen Erhaltungstherapie behandelten erwachsenen Asthmapatienten ist eine (freie oder) fixe Kombination eines niedrig dosierten inhalativen Glucocorticosteroids mit einem langwirksamen Beta-2-Sympathomimetikum zur initialen Langzeittherapie. Im Vergleich mit einer niedrigen Dosis inhalativer Glucocorticosteroide verbessert eine ICS/LABA-Therapie die Asthmasymptomatik und die Lungenfunktion. Das Exazerbationsrisiko wird im Vergleich zu einer ICS-Monotherapie allerdings nicht zusätzlich reduziert [234]. Für Kinder und Jugendliche wird die Kombinationstherapie erst ab Stufe 3 empfohlen; in Einzelfällen kann bei Jugendlichen eine Kombination ICS mit LABA bereits bei Stufe 2 sinnvoll sein [235].

Bei Patienten mit ausschließlich saisonalem allergischem Asthma (z. B. Sensibilisierung gegen Baum- und/oder Gräserpollen) ohne Asthmabeschwerden außerhalb der Allergensaison kann eine Therapie mit ICS unmittelbar bei Auftreten erster Asthmasymptome bei Allergenexposition begonnen werden. Die ICS-Therapie sollte noch für 4 Wochen nach Ende der Allergensaison fortgeführt werden.

Theophyllin-Präparate mit verzögerter Wirkstoff-Freisetzung sind nur schwach antiasthmatisch wirksam [207,236243] und weisen ein im Vergleich zu den bevorzugt empfohlenen Langzeittherapeutika hohes Nebenwirkungsrisiko auf [244]. Chromone (Nedocromil und Chromoglyzinsäure) sind sehr gut verträgliche Medikamente mit allerdings niedriger antiasthmatischer Effektivität [245,246]. Für Chromone und Theophyllin gibt es bei Kindern und Jugendlichen mit Asthma bronchiale auch im Rahmen einer Stufe-2-Therapie keine Indikation. Beide Präparate sollten auch bei Erwachsenen nur in begründeten Ausnahmefällen zur antiasthmatischen Therapie in Stufe 2 eingesetzt werden.

Zusätzlich zur entzündungshemmenden Langzeittherapie sollte auch in Therapiestufe 2 zur Symptomkontrolle ein kurzwirksames inhalatives Beta-2-Sympathomimetikum nur bei Bedarf angewandt werden. Eine regelmäßige oder sogar tägliche Anwendung eines solchen Präparats deutet auf eine unzurei- chende Asthmakontrolle hin. In solchen Fällen sollte die Therapieintensität gesteigert und die Patienten ggf. nach den Empfehlungen der nächsthöheren Therapiestufe behandelt werden.

\subsubsection{Therapiestufe 3: 2 Langzeittherapeutika plus raschwirksames Bedarfstherapeutikum}

Als bevorzugte Therapieoptionen soll erwachsenen Patienten in Therapiestufe 3 eine regelmäßige Langzeittherapie mit

1. einer fixen Kombination eines niedrig dosierten ICS mit einem LABA und die bedarfsweise Anwendung eines kurzwirksamen inhalativen Beta-2-Sympathomimetikums (SABA) ODER

2. eine fixe Kombination eines niedrig dosierten ICS (Budesonid oder Beclomethason) mit dem LABA Formoterol zur

Langzeit- und Bedarfstherapie empfohlen werden.

Für Kinder und Jugendliche ist in Therapiestufe 3 die bevorzugte Therapieoption eine inhalative Corticosteroidtherapie in zunächst unterer mittelhoher Dosis. Wenn darunter keine gute Asthmakontrolle zu erreichen ist, wird die Therapie um ein LABA oder LTRA erweitert (Stufe 4).

Patienten, deren Asthma mit einer täglichen Therapie nur mit einem inhalativen Glucocorticosteroid in niedriger Dosis nicht zu kontrollieren ist, wird entweder eine regelmäßige Langzeittherapie mit einer (freien oder) fixen Kombination eines niedrig dosierten ICS mit einem LABA und die bedarfsweise Anwendung eines kurzwirksamen inhalativen Beta-2-Sympathomimetikums (SABA) oder eine fixe Kombination eines niedrig dosierten ICS mit dem LABA Formoterol zur Langzeit- und Bedarfstherapie ((S)MART: (Single inhaler) Maintenance And Reliever Therapy) empfohlen. Zur Behandlung in Therapiestufe 3 stehen freie und fixe ICS/LABA-Kombinationen aus Fluticasonpropionat/Formoterol, Fluticasonpropionat/Salmeterol, Beclomethason/Formoterol und Budesonid/Formoterol sowie zur ausschließlich einmal täglichen Applikation Fluticasonfuroat/ Vilanterol nur in fixer Kombination zur Verfügung. Zur Langzeitund Bedarfstherapie ((S)MART) können die fixen Beclometason/Formoterol- und Budesonid/Formoterol-Kombinationen verwendet werden, jeweils mit den ICS-Komponenten in niedriger Dosis (s. Tab.8). Im Kindesalter stehen allerdings nur die Kombinationen Budesonid/Formoterol und Fluticason/Salmeterol als zugelasssene Präparate zur Verfügung.

Die gleichzeitige Therapie mit ICS und LABA verringert im Vergleich zur Langzeittherapie nur mit ICS die Asthmasymptomatik einschließlich nächtlicher Asthmabeschwerden und den Bedarf an raschwirksamen Beta-2-Sympathomimetika zur akuten Symptomkontrolle, verbessert die Lungenfunktion und reduziert die Exazerbationsfrequenz [210, 211, 247 - 252]. Bei Patienten mit erhöhtem Exazerbationsrisiko verringert eine Langzeit- und Bedarfstherapie mit fixen ICS/Formoterol-Kombinationen signifikant die Exazerbationsfrequenz und erhält die Asthmakontrolle mit im Verhältnis niedrigeren ICS-Dosen, jeweils im Vergleich mit einer fixen ICS/LABA-Kombination nur zur Langzeittherapie oder einer höheren ICS-Dosis zur Langzeittherapie und jeweils mit einem SABA bei Bedarf [253 - 257]. 
Generell gilt, dass fixe ICS/LABA-Kombinationen zumindest so effektiv wie die Einzelkomponenten sind [258-263]. Für Kinder und Jugendliche ist gezeigt worden, dass mit einer fixen Kombination eine bessere Asthmakontrolle zu erreichen ist [264].

Diese Art der Medikation ist für den Patienten nicht nur bequem, sondern führt auch zu einer verbesserten (vor allem ICS-)Adhärenz und zu einer erhöhten Therapiesicherheit durch Verhinderung einer LABA-Monotherapie. Allerdings ist die wünschenswerte Flexibilität bei der Wahl der Dosis sowohl des inhalativen Glucocorticosteroids als auch des langwirksamen Beta2-Sympathomimetikums damit schwieriger umzusetzen.

Bevor eine Behandlung in einer höheren Therapiestufe erwogen wird, sollten immer die Inhalationstechnik, die Therapietreue und die Exposition gegenüber (bislang unerkannten) inhalativen Noxen bedacht und im Zweifel die asthmatische Genese der Beschwerden bestätigt werden.

Eine im Vergleich zu einer Therapie mit ICS und LABA weniger häufig effektive Therapiealternative in Stufe 3 sind ICS in mittlerer Dosis $[213,265,266]$. Bei Erwachsenen ist in den meisten Fällen die Addition eines zweiten Wirkstoffes der Erhöhung der Dosis bei Monotherapie vorzuziehen [249, 267, 268]. Die Beziehung zwischen ICS-Dosis und -Wirkung auf die meisten Parameter der Asthmakontrolle ist relativ flach [269]. Mit steigenden ICS-Dosen nimmt die zusätzliche antiasthmatische Wirkung ab und das Risiko von Nebenwirkungen zu [213,266, 270]. Allerdings besteht eine klare Beziehung zwischen der Höhe der ICS-Dosis und der Prävention schwerer Exazerbationen [249].

Weitere im Vergleich zu einer Therapie mit ICS und LABA weniger häufig effektive Therapiealternativen in Stufe 3 sind eine niedrige ICS-Dosis in Kombination mit entweder einem Leukotrienrezeptor-Antagonisten [227, 228, 230-232, 271-275] oder bei Erwachsenen in begründeten Fällen mit einer niedrigen Dosis eines Theophyllin-Präparates mit verzögerter Wirkstoff-Freisetzung [207, 229, 233, 238].

Unter einer Langzeitbehandlung mit einem Theophyllin-Präparat mit verzögerter Wirkstoff-Freisetzung sollte die Theophyllin-Serumkonzentration zwischen 5 und $15 \mathrm{mg} / \mathrm{l}$ liegen (Bestimmung am Morgen vor der Theophyllin-Einnahme) [207]. Für Kinder und Jugendliche ist Theophyllin auch in Stufe 3 keine Therapieoption. Langwirksame orale Beta-2-Sympathomimetika können ähnlich wirksam sein wie langwirksame inhalative Beta-2-Sympathomimetika [276-278]. Allerdings steigt durch die enterale Gabe das Risiko unerwünschter Nebenwirkungen an.

Prinzipiell kann auch das langwirksame Anticholinergikum Tiotropium als Alternative zu einem LABA in freier Kombination mit einem ICS in Stufe 3 eingesetzt werden, z. B. bei schweren LABA-Nebenwirkungen. Tiotropium ist in dieser Indikation zwar nicht in Deutschland, aber in anderen Ländern zugelassen.
5.1.5 Therapiestufe 4: 2 oder mehr Langzeittherapeutika plus raschwirksames Bedarfstherapeutikum

Als bevorzugte Therapieoptionen soll erwachsenen Patienten in Therapiestufe 4

1. eine fixe Kombination eines niedrig dosierten ICS mit dem LABA Formoterol zur Langzeit- und Bedarfstherapie. ODER

2. eine regelmäßige Langzeittherapie mit einer fixen Kombination einer mittleren oder hohen ICS-Dosis mit einem LABA und die bedarfsweise Anwendung eines kurzwirksamen inhalativen Beta-2-Sympathomimetikums (SABA) empfohlen werden.

Für Kinder und Jugendliche gilt, dass bei unzureichender Therapiekontrolle unter einem mittelhochdosierten ICS eine Kombination aus ICS und LABA am häufigsten wirksam ist. Alternativ kann eine Kombination aus ICS und LTRA versucht werden oder die ICS-Dosis verdoppelt werden. Bei der Kombination aus ICS und LABA soll die ICS-Dosis wenigstens im oberen Bereich der Niedrigdosis oder im unteren Bereich der mittelhohen Dosis nach pädiatrischen Dosisangaben liegen. Wenn die Zugabe des LABA nicht zu einer Verbesserung der Asthmakontrolle führt, soll die LABA-Therapie wieder beendet und eine alternative Option versucht werden. Wenn die Kombination mit LABA zu einer Verbesserung der Kontrolle, aber nicht zu einer guten Kontrolle des Asthmas führt, soll die ICS-Dosis erhöht werden [279].

Für Kinder und Jugendliche gilt, dass bereits bei unzureichender Asthmakontrolle unter einer Stufe-3-Therapie die Vorstellung in einem spezialisierten Zentrum/bei einem Kinderpneumologen zur Mitbeurteilung und ggf. weiteren differenzialdiagnostischen Klärung erwogen werden sollte.

Die Art der Eskalation der Therapieintensität in Stufe 4 wird bei Erwachsenen ggf. durch die Therapieentscheidung in Stufe 3 beeinflusst. Für Patienten mit $\geq 1$ Exazerbation im letzten Jahr ist eine fixe Kombination eines niedrig dosierten ICS mit dem LABA Formoterol zur Langzeit- und Bedarfstherapie ((S) MART-Therapie) bei vergleichbarem Effekt auf die Asthmakontrolle effektiver zur Reduktion der Exazerbationsfrequenz als die gleiche ICS/LABA-Dosis nur zur Langzeittherapie oder höhere ICS-Dosen [253]. Zur (S)MART-Therapie zugelassen sind Budesonid/Formoterol- und Beclomethason-Kombinationen (vgl. Therapiestufe 3), wobei die Dosierung der Langzeittherapie bei erhöhter Frequenz der Bedarfsanwendung gesteigert werden kann. Bei Patienten mit ungenügender Asthmakontrolle unter niedrig dosierter ICS/LABA-Langzeittherapie plus SABA ist eine Therapieeskalation durch eine mittlere ICS/LABA-Dosis möglich [280]. Zur Behandlung in Therapiestufe 4 stehen freie und fixe ICS/LABA-Kombinationen aus Fluticasonpropionat/ Formoterol, Fluticasonpropionat/Salmeterol, Beclomethason/ Formoterol und Budesonid/Formoterol sowie zur ausschließlich einmal täglichen Applikation Fluticasonfuroat/Vilanterol nur in fixer Kombination zur Verfügung.

Eine Option zur Therapieeskalation ist Tiotropium im Sprühvernebler als additive bronchodilatatorische Langzeittherapie für Erwachsene mit ungenügender Asthmakontrolle trotz Behandlung mit ICS ( $\geq 800 \mu \mathrm{g}$ Budesonid pro Tag oder Äquivalent) und LABA sowie $\geq 1$ Exazerbation im letzten Jahr [281-283]. 
Für Kinder und Jugendliche liegen Daten zur Wirksamkeit und Verträglichkeit von Tiotropium vor [284-286], es besteht aber derzeit (Stand 2017) keine Zulassung in Deutschland.

Weitere Optionen zur Therapieeskalation sind hochdosierte ICS/LABA-Kombinationen, obwohl die Steigerung der ICS-Dosis einen meist nur geringen zusätzlichen Therapieeffekt bewirkt [70, 213, 249, 266], bei einem erhöhten Risiko von Nebenwirkungen. Eine hochdosierte ICS/LABA-Kombination kann versuchsweise für 3-6 Monate eingesetzt werden, wenn mit einer mittleren ICS/LABA-Kombination und einem 3. Langzeittherapeutikum (Tiotropium, Montelukast oder einem TheophyllinPräparat mit verzögerter Wirkstoff-Freisetzung) keine ausreichende Asthmakontrolle erzielt werden kann [241,271,275, 281 - 283, 287]. Die Wirksamkeit des ICS Budesonid in mittlerer und hoher Dosis kann im Vergleich zur zweimal täglichen durch viermal tägliche Inhalation gesteigert werden, eine adäquate Adhärenz vorausgesetzt [288,289]. Für alle anderen ICS wird eine zweimal tägliche Applikation empfohlen. Neben einem LABA können mit geringerer Effektstärke mittel- und hochdosierte ICS auch mit Montelukast [273-275, 290,291] oder einem Theophyllin-Präparat mit verzögerter Wirkstoff-Freisetzung kombiniert werden [241]. Die Definition einer hochdosierten ICS-Therapie bei Erwachsenen ist derzeit international uneinheitlich: Der Konsensus der internationalen Fachgesellschaften (ERS/ATS 2014 [128]) ( Tab.9) empfiehlt höhere ICS-Dosierungen als die GINA (2017) [2] ( Tab. 8). Die seitens des ERS/ATS-Konsensus vorgeschlagenen Grenzwerte für eine ICS-Hochdosis bei Erwachsenen ( Tab.9) erscheinen sinnvoller, da sie den zugelassenen Maximaldosierungen und der klinischen Realität am ehesten entsprechen. Es wird daher bei Erwachsenen empfohlen, sich am Konsensus der internationalen Fachgesellschaften (ERS/ATS 2014 [128]) (\ Tab.9) zu orientieren.

\subsubsection{Therapiestufe 5: Additive Therapie}

Asthmapatienten mit persistierenden Symptomen und Exazerbationen unter einer Stufe-4-Therapie (ICS plus $\geq 2$ Controller) trotz adäquater Therapietreue und korrekter Inhalationstechnik sollen eine Evaluation durch einen im Management des schweren Asthmas erfahrenen Pneumologen und additive Therapieoptionen auf der Grundlage einer fixen ICS/LABA-Kombination empfohlen werden. Kinder und Jugendliche sollen bei V.a. schweres persistierendes Asthma in einem spezialisierten Zentrum mit Möglichkeit zur invasiven Diagnostik und zur Beurteilung in einem multiprofessionellen Team vorgestellt werden [128].

Die Diagnose eines schweren Asthmas bei Patienten unter Therapie mit ICS in hoher Dosis und mindestens einem zusätzlichen Controller (LABA, Montelukast oder Theophyllin) oder oralen Glukokortikoiden über mehr als 6 Monate pro Jahr setzt mindestens einen der folgenden Befunde voraus bzw. einer dieser Befunde würde bei Reduktion der Therapieintensität mit Wahrscheinlichkeit eintreten [127, 128]:

- nicht gut kontrolliertes Asthma

- Atemwegsobstruktion (z. B. FEV $1<$ LLN bei FEV $1 / F V C<L L N$ )

- häufige Exazerbationen (z. B. $\geq 2$ Glucocorticoid-pflichtige

Exazerbationen in den letzten 12 Monaten)
- schwere Exazerbationen (z. B. $\geq 1$ Exazerbation mit stationärer Behandlung oder Beatmung in den letzten 12 Monaten)

Die Erkrankung von Patienten mit schwerem persistierendem Asthma kann oft dauerhaft nicht vollständig kontrolliert werden. Ziel der Therapie ist es daher, die bestmöglichen Therapieergebnisse bei möglichst geringen Nebenwirkungen der Medikation zu erzielen. Die Therapie besteht meist aus einer Kombination mehrerer täglich anzuwendender Antiasthmatika. In Therapiestufe 5 können, sofern nicht bereits ohne Erfolg in Therapiestufe 4 erprobt, neben inhalativen Glucocorticosteroiden und langwirksamen inhalativen Beta-2-Sympathomimetika weitere Asthmatherapeutika eingesetzt werden:

- Tiotropium im Sprühvernebler als bronchodilatatorische Langzeittherapie additiv zur Behandlung mit ICS ( $\geq 800 \mu \mathrm{g}$ Budesonid pro Tag oder Äquivalent) und LABA für Erwachsene mit $\geq 1$ Exazerbation im letzten Jahr verbessert die Lungenfunktion und verlängert die Zeit bis zur nächsten Exazerbation [281, 282]. Eine Zusatztherapie mit Tiotropium ist keine zwingende Voraussetzung für den Einsatz von Biologika bei schwerem Asthma, ein Therapieversuch sollte jedoch angestrebt werden.

- Omalizumab, ein monoklonaler humanisierter, gegen Immunglobulin E gerichteter Antikörper wird für Kinder ab 6 Jahren, Jugendliche und Erwachsene mit schwergradigem allergischem Asthma empfohlen, das durch eine Stufe-4Therapie nicht adäquat zu kontrollieren ist [128, 292]. Omalizumab bindet an zirkulierendes freies IgE und senkt die Expression von IgE-Rezeptoren auf Effektorzellen, dies führt zu einer selektiven Hemmung IgE-vermittelter Reaktionen. Bei Patienten mit schwerem, durch Standardtherapie nicht ausreichend kontrollierbaren allergischem Asthma ließ sich unter Omalizumab ein Rückgang der klinischen Beschwerden, des Medikamentenverbrauchs und der Anzahl an Asthmaexazerbationen nachweisen, während gleichzeitig die Lebensqualität verbessert wurde. Omalizumab ist eine zusätzliche Option zur Verbesserung der Asthmakontrolle bei Erwachsenen, Jugendlichen und Kindern ab dem 7. Lebensjahr mit schwerem persistierendem allergischem Asthma, die einen positiven Hauttest oder In-vitro -Reaktivität gegen ein ganzjährig auftretendes Aeroallergen zeigen und sowohl eine reduzierte Lungenfunktion $\left(\mathrm{FEV}_{1}<\mathrm{LLN}\right.$; Ausnahme: Bei Kindern $<12$ Jahre ist die Indikation unabhängig von der Lungenfunktion) haben als auch unter häufigen Symptomen während des Tages oder nächtlichem Erwachen leiden und trotz täglicher Therapie mit einem hoch dosierten inhalativen Glucocorticosteroid und einem lang wirkenden inhalativen $\beta_{2}$-Agonisten mehrfach dokumentierte, schwere Asthmaexazerbationen hatten. Nicht alle Patienten mit schwerem allergischen Asthma, die die genannten Kriterien erfüllen, sprechen auf eine Omalizumab-Therapie an. Der Therapieerfolg sollte daher nach einer Behandlung mit Omalizumab über mindestens vier Monate von in der Diagnose und Therapie des schweren persistierenden Asthmas erfahrenen Ärzten beurteilt werden, danach in jährlichen Intervallen. Omalizumab kann auch bei Patienten ohne Allergienachweis wirksam sein [293], ist dafür aber derzeit 
nicht zugelassen. In begründeten Einzelfällen und bei fehlenden Therapiealternativen kann ein Therapieversuch in einem im Management des schweren refraktären Asthmas erfahrenen Zentrum erwogen werden.

- Mepolizumab und Reslizumab, monoklonale, gegen Interleukin-5 gerichtete Antikörper, werden für $\geq 18$ Jahre alte Patienten mit schwergradigem eosinophilen Asthma empfohlen, das durch eine Stufe-4-Therapie nicht adäquat zu kontrollieren ist [101, 108, 109, 294 -296]. Mepolizumab und Reslizumab binden zirkulierendes freies Interleukin-5 und führen so zu einer effektiven Inhibition der Reifung und Aktivierung eosinophiler Granulozyten, mit konsekutiver Reduktion der Zahl eosinophiler Granulozyten im peripheren Blut und in der Lunge. Bei Patienten mit durch eine Standardtherapie mit ICS und LABA nicht adäquat kontrolliertem eosinophilen Asthma führen Mepolizumab und Reslizumab zu einem Rückgang der Exazerbationsfrequenz, der Asthmasymptome und des Bedarfs an systemischen Glucocorticosteroiden bei gleichzeitiger Verbesserung der Lungenfunktion, der Asthmakontrolle und der Asthma-spezifischen Lebensqualität [101, 108, 109, 294 - 296]. Mepolizumab wird subkutan in einer Dosis von $100 \mathrm{mg}$ einmal monatlich injiziert, Reslizumab in einer Dosis von $3 \mathrm{mg}$ pro Kilogramm Körpergewicht ebenfalls einmal monatlich intravenös als Infusion verabreicht. Die Kriterien zur Definition eines „eosinophilen“ Asthmas unterschieden sich in den verschiedenen Zulassungsstudien und schwanken zwischen $\geq 150$ Eosinophilen/ $\mu$ l Blut bei Studieneinschluss bei

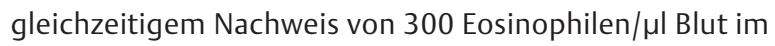
letzten Jahr (Mepolizumab) und $\geq 400$ Eosinophilen/ $\mu$ l Blut (Reslizumab). Ein mindestens zweimaliger Nachweis von mehr als 300 Eosinophilen pro $\mu$ l Blut in den letzten 12 Monaten außerhalb von Exazerbationen sollte angestrebt werden, um das Vorhandensein eines eosinophilen Asthmas zu verifizieren. Im Rahmen der Indikationsstellung ist zu berücksichtigen, dass eine Therapie mit systemischen Glucocorticoiden die Zahl der eosinophilen Granulozyten im Blut verringert. Die Behandlung mit Mepolizumab oder Reslizumab sollte von in der Diagnose und Therapie des schweren persistierenden Asthmas erfahrenen Ärzten begonnen werden. Nicht alle Patienten mit schwerem eosinophilen Asthma profitieren von einer gegen Interleukin-5 gerichteten Therapie. Die Wirksamkeit einer Behandlung mit den monoklonalen Antikörpern Mepolizumab oder Reslizumab soll nach vier und zwölf Monaten, danach in jährlichem Abstand evaluiert werden. Für Kinder und Jugendliche $<12$ Jahre liegen keine ausreichenden Daten zu Sicherheit und Wirksamkeit vor.

Nach Beginn einer Behandlung mit monoklonalen Antikörpern soll die bisherige inhalative und orale Asthmatherapie für mindestens 4 Wochen beibehalten und erst danach unter engmaschiger Beurteilung der Asthmakontrolle ggf. reduziert werden.

Eine intermittierende oder dauerhafte additive Therapie mit niedrigen Dosen oraler Glucocorticosteroide ( $\leq 7,5 \mathrm{mg}$ Prednison oder Äquivalent) kann für Patienten mit schwerem Asthma nach erfolglosem Einsatz der anderen in Stufe 5 empfohlenen Therapieoptionen in Betracht gezogen werden [128, 297, 298], wobei das Risiko schwerwiegender Nebenwirkungen besteht [299]. Orale Glucocorticosteroide sollten nur bei Patienten mit schlechter Symptomkontrolle und/oder Exazerbationen trotz Therapie nach Stufe 4 bei guter Adhärenz und korrekter Inhalationstechnik sowie nach Ausschluss von die Asthmakontrolle beeinträchtigenden Kofaktoren eingesetzt werden, und dann in der niedrigsten noch effektiven Dosis, vorzugsweise als Einzeldosis am Morgen, um systemische Nebenwirkungen zu minimieren [300, 301]. Die Patienten müssen über typische Nebenwirkungen einer Therapie mit systemischen Glucocorticosteroiden aufgeklärt werden. Eine regelmäßige Verlaufsbeurteilung auch im Hinblick auf eine Glucocorticosteroid-induzierte Osteoporose ist erforderlich, unter Einschluss einer umfassenden Beratung zur optimalen Osteoporose-Prävention bei zu erwartender Therapiedauer von $\geq 3$ Monaten. Patienten unter Dauertherapie mit systemischen Glucocorticosteroiden sollen eine Osteoporose-Prophylaxe durchführen [302,303]. Bei Beendigung der Therapie mit oralen Glucocorticosteroiden bzw. beim Wechsel von oralen Glucocorticosteroiden auf hochdosierte inhalative Glucocorticosteroide müssen die Patienten engmaschig überwacht und die Möglichkeit einer temporären oder dauerhaften Nebenniereninsuffizienz in Betracht gezogen werden. Bei schwer zu kontrollierendem Asthma mit nächtlichen Beschwerden kann die Tagesdosis des systemischen Corticoids auf 2/3 am Morgen und 1/3 der Dosis am Nachmittag verteilt werden. Für Kinder und Jugendliche stellt die regelmäßige systemische Glucocorticosteroid-Therapie die letzte Therapieoption dar; sie sollte nur nach ausführlicher Evaluation des Patienten in einem in der Behandlung von schwerem Asthma erfahrenen Zentrum nach differenzialdiagnostischer Klärung und Ausschluss aggravierender Faktoren in Betracht gezogen werden. Alle Maßnahmen zur Allergenreduktion (einschließlich stationäre Rehabilitationsmaßnahme und Einsatz eines Luftreinigers mit laminarem Fluss) sollten ausgeschöpft sein. In der Regel ist ein Behandlungsversuch mit Omalizumab bereits erfolgt.

Mit einem Vernebler können nominell höhere Dosen inhalativer Glucocorticosteroide (Budesonid oder Fluticason) appliziert werden. Allerdings deuten nur wenige Befunde darauf hin, dass eine solche Therapie effektiver als eine konventionelle inhalative Therapie ist [304] oder dass es dadurch zu geringeren systemischen Nebenwirkungen im Vergleich zu einer äquivalenten Dosis eines oralen Glucocorticosteroids kommt [305, 306]. Zudem ist diese Art der Behandlung im Verhältnis zur Therapie mit Dosieraerosolen bzw. Pulverinhalatoren kostenaufwendiger und mit dem erhöhten Risiko lokaler Nebenwirkungen (Heiserkeit, Soor) behaftet. Es liegen keine kontrollierten klinischen Prüfungen vor, die eine Überlegenheit der über einen Vernebler applizierten gegenüber oralen Glucocorticosteroiden nachweisen. Für die Langzeittherapie kann die Verneblung von Glucocorticosteroiden nicht empfohlen werden.

Glucocorticosteroid-sparende Therapiealternativen (z.B. Methotrexat $[128,307])$ können in begründeten Fällen bei Patienten mit schwerem persistierenden Asthma in Betracht gezogen werden, die dauerhaft auf orale Glucocorticosteroide an- 
gewiesen sind und unter systemischen Nebenwirkungen leiden $[128,308]$. Die therapeutische Wirksamkeit dieser Behandlungen ist jedoch individuell ausgeprägt und geht mit zum Teil erheblichen Nebenwirkungen einher [128, 309, 310]. Glucocorticosteroid-sparende Therapieverfahren können daher nicht generell empfohlen werden und sollten nur bei eindeutiger klinischer Wirksamkeit längerfristig weitergeführt werden, und nur unter strenger ärztlicher Kontrolle durch einen erfahrenen, auf die Behandlung obstruktiver Atemwegserkrankungen spezialisierten Arzt.

Die bronchiale Thermoplastie kann für ausgewählte erwachsene Patienten mit schwerem Asthma in Betracht gezogen werden [128]. Die Erfahrungen mit dieser Technik sind begrenzt, die Langzeiteffekte unbekannt (vgl. 7.8).

Komplexe medikamentöse Therapieempfehlungen führen häufig zu mangelnder Therapietreue des Patienten, was wiederum den zur Kontrolle des Asthmas erforderlichen medikamentösen und nicht medikamentösen Aufwand erhöhen kann. Patienten mit schwergradigem persistierendem Asthma sollten deswegen besonders intensiv in allen Aspekten des medikamentösen und nicht medikamentösen Managements ihrer Erkrankung geschult werden.

Die zuverlässige Einstufung eines Asthmapatienten als Patient mit schwerem oder schwer zu behandelndem Asthma kann nicht gestützt auf eine einzige Untersuchung erfolgen, selbst nicht durch einen in der Behandlung von Asthma erfahrenen Arzt. Besonders bei schwierig zu behandelndem bzw. therapierefraktärem Asthma soll die Diagnose Asthma eindeutig gesichert sein. Zum Ausschluss anderer Krankheiten bzw. zur Erfassung zusätzlicher Erkrankungen ist eine gründliche systematische Abklärung notwendig. Diese Untersuchungen erfordern u. U. einen stationären Aufenthalt des Patienten in einem auf Asthma spezialisierten Zentrum. Zudem soll die Therapietreue des Patienten als einwandfrei dokumentiert werden [311].

\section{2 (Allergen-)spezifische Immuntherapie (SIT)}

\section{EMPFEHLUNGEN}

E28 Die subkutane Immuntherapie (SCIT) und sublinguale Immuntherapie (SLIT) können bei stabilem allergischem Asthma ( $\mathrm{FEV}_{1}>70 \%$ bei Erwachsenen) als Therapieoption neben Allergenkarenz und Pharmakotherapie durchgeführt werden, wenn

- die allergische Ursache der Asthmaerkrankung eindeutig festzustellen ist (Anamnese, Nachweis der lgE-vermittelten Reaktion, deutliche Hinweise auf kausalen Zusammenhang mit Asthmasymptomen)

- Allergenkarenz nicht zur Asthmakontrolle führt oder nicht möglich ist.

E29 Die SCIT und SLIT sollen bei unkontrolliertem bzw. schwergradigem Asthma oder bei Erwachsenen mit FEV $\leq 70 \%$ des Sollwertes nicht eingesetzt werden.

S3 Die Immuntherapie ist kein Ersatz für eine wirksame antiasthmatische Pharmakotherapie.
Die Indikation zur spezifischen Immuntherapie (SIT) bei allergischem Asthma bronchiale wird insgesamt zurückhaltender gestellt als bei der allergischen Rhinokonjunktivitis. Die SIT kann eine ausreichende antiasthmatische Therapie nicht ersetzen [312]. Allerdings ist der Einsatz bei auf Therapiestufe 1 oder 2 kontrolliertem Asthma bronchiale [2] als Therapieoption neben Allergenkarenz und Pharmakotherapie zu empfehlen. Die Voraussetzung hierfür ist ein eindeutiger kausaler Zusammenhang zwischen respiratorischen Symptomen und entsprechendem Allergen sowie der Einsatz von Präparaten, deren Wirksamkeit bei asthmatischen Patienten durch kontrollierte klinische Studien belegt ist $[97,312,313]$. Ein unkontrolliertes Asthma bronchiale stellt eine Kontraindikation für die SIT dar.

Eine mehrfach aktualisierte Metaanalyse der Cochrane Library hat 201088 randomisierte kontrollierte, methodisch allerdings heterogene SCIT-Studien mit insgesamt 3459 asthmatischen Patienten ausgewertet [314]. Insgesamt fand sich eine signifikante Reduktion des Symptomscores sowie des Medikamentenverbrauchs sowie eine leichte, aber signifikante Reduktion der unspezifischen bronchialen Hyperreaktivität. Die Auswertung der 20 Studien, die im Verlauf der SCIT die Lungenfunktionsparameter gemessen haben, ergab nur einen Trend für die Verbesserung der Werte ohne statistische Signifikanz. Eine separate Analyse von pädiatrischen Studien wurde in dieser Cochrane-Auswertung nicht durchgeführt.

Eine andere, aktuellere Metaanalyse hat 19 Studien (hiervon drei Studien ausschließlich an Kindern) ausgewertet, in welchen die Wirksamkeit der SCIT bei (Hausstaubmilben-) allergischem Asthma bronchiale untersucht worden ist [315]. Hierbei konnten die Autoren nur in neun Studien eine statistisch signifikante Überlegenheit der SCIT gegenüber der Placebobehandlung bei den Symptomscores finden. Allerdings betonten die Autoren eine hohe Heterogenität der Studien bezüglich der eingesetzten Allergendosen sowie der gewählten Zielparameter und Auswertezeiträume (Übersicht in [312]). Eine neuere Studie hat die Wirksamkeit der SCIT mit einem Milbenallergoid an Kindern mit primärem allergischen Asthma untersucht und fand eine verbesserte Kontrolle des Asthmas bei signifikant reduzierter Dosis an inhalativen Glucocorticosteroiden bei den SCIT-therapierten Kindern im Vergleich zur Kontrollgruppe ohne SCIT [316].

Einige Studien an Kindern und Jugendlichen haben sekundärpräventive, krankheitsmodifizierende Effekte der SCIT beschrieben. Bei alleiniger allergischer Rhinitis konnte beispielsweise durch die SCIT die Ausbildung von Asthmasymptomen i. S. eines Etagenwechsels bis zu 7 Jahre nach Beendigung der dreijährigen Therapie reduziert werden [317].

Auch bei der sublingualen Immuntherapie (SLIT) wird die Indikation bei Asthma zurückhaltender gestellt als bei der allergischen Rhinokonjunktivitis. Nur wenige Studien zur Wirksamkeit bei Asthma bronchiale liegen vor. Auch bei der SLIT ist die Voraussetzung ein eindeutiger kausaler Zusammenhang zwischen respiratorischen Symptomen und entsprechendem Allergen sowie der Einsatz von SLIT-Präparaten, deren Wirksamkeit bei asthmatischen Patienten durch kontrollierte klinische Studien belegt ist [312]. Auch die SLIT kann eine antiasthmatische Therapie nicht ersetzen [312]. Allerdings hat sich beispielsweise in 
einer Studie zur SLIT mit Gräserpollen-Tabletten gezeigt, dass sich die Asthmasymptome in der Subgruppe der asthmatischen Kinder und Jugendlichen verbessert haben [318].

Die Studienergebnisse zur Wirksamkeit der SLIT mit Milbenextrakten bei allergischem Asthma sind teilweise heterogen und mit methodischen Mängeln behaftet ([319, 320], Übersicht in [315]). Aktuelle Studien haben jedoch gezeigt, dass eine SLIT bei erwachsenen Patienten mit Asthma und Hausstaubmilbenallergie und begleitender allergischer Rhinitis den ICS-Bedarf und die Exazerbationsrate senken kann [97,313]. Diese Therapie war auch bei Patienten mit teilkontrolliertem Asthma sicher, daher stellt ein teilkontrolliertes Asthma in diesem Falle keine Kontraindikation dar [97]. Zulassungen für eine SLIT bei Kindern und Jugendlichen mit teilkontrolliertem Asthma und Hausstaubmilbenallergie liegen aktuell nicht vor. Inwieweit sich bei Kindern durch die SLIT auch der Etagenwechsel im Sinne eines sekundärpräventiven Therapieeffektes verhindern lässt, wird derzeit in einer großen, multizentrischen Studie untersucht [321]. Ein unkontrolliertes Asthma bronchiale stellt auch bei der SLIT eine Kontraindikation dar [312].

Die Hochdosis-SLIT führte bei Patienten mit Rhinokonjunktivitis und Asthma bei Hausstaubmilbenallergie [313] neben einem signifikanten Therapieerfolg bei der Rhinokonjunktivitis auch zu einer signifikanten Abnahme des ICS-Verbrauchs, wobei in der Gruppe der teilkontrollierten Patienten mit Asthma eine höhere ICS-Reduktion nachgewiesen wurde. Die Hochdosis-SLIT führte bei Asthmapatienten, die unter ICS-Therapie nur teilkontrolliert waren (und somit nicht mehr der TherapieStufe 1 oder 2 zuzuordnen sind), zu keinen respiratorischen $\mathrm{Ne}$ benwirkungen. Es konnte bei diesen Patienten eine Abnahme der Asthmaexazerbationen gezeigt werden [97]. Aufgrund dieser Daten hat die GINA-Leitlinie (2017) eine Hochdosis-Hausstaubmilben-SLIT auch für erwachsene Asthmapatienten der Therapiestufe 3 und 4 als Therapieoption beschrieben [2].

Zusammenfassend sollte die SIT bei auf Therapiestufe 1 oder 2 kontrolliertem IgE-vermitteltem allergischem Asthma bronchiale als Therapieoption neben Allergenkarenz und Pharmakotherapie zur Anwendung kommen mit Produkten, für welche entsprechende Effekte in kontrollierten klinischen Studien gezeigt worden sind (Übersicht bei [322]). Insbesondere sekundärpräventive Aspekte wie z. B. ein vermindertes Asthmarisiko bei bestehender allergischer Rhinokonjunktivitis stellen wichtige Gründe für einen möglichst frühzeitigen Beginn der Therapie im Kindes- und Jugendalter dar.

\subsection{Inhalationssysteme - Verordnung, Technik, Training}

Im klinischen Alltag ist die Fehlerquote in der Inhalationstechnik hoch. Es gibt zahlreiche Inhalationssysteme, die in ihrer Handhabung völlig unterschiedlich sind. Jedes Inhalationsgerät erfordert ein individuelles Inhalationsmanöver und stellt bestimmte systemeigene Anforderungen an die korrekte Inhalation. Der Patient soll vor der Erstanwendung intensiv in die Handhabung des Inhalationsgerätes durch einen Arzt oder geschultes Fachpersonal eingewiesen werden. Ein alleiniger Verweis auf den Beipackzettel ist nicht ausreichend. Die Hinweise des Herstellers für die Handhabung des jeweiligen Inhalations-

\section{EMPFEHLUNGEN}

E30 Die Auswahl eines geeigneten Inhalationssystems soll in Übereinstimmung mit dem Patienten erfolgen.

E31 Vor Verschreibung eines Inhalationssystems soll der Patient in dessen Gebrauch unterwiesen werden und die korrekte Handhabung demonstriert und geschult werden.

E32 Die Inhalationstechnik des Patienten soll regelmäßig überprüft werden.

E33 Bei Wechsel eines Inhalationssystems soll eine Neueinweisung des Patienten erfolgen und die Notwendigkeit einer Dosisanpassung geprüft werden.

E34 Wenn möglich, sollte für die Langzeittherapie ein Inhalationssystem (nur ein Typ eines Dosieraerosols oder eines Pulverinhalators) für alle erforderlichen Medikamente verordnet werden.

E35 Bei stationärer Behandlung des Asthmapatienten soll die Inhalationstherapie fortgeführt werden (Dosieraerosole, ggf. mit Spacer oder Vernebler). Bei Patienten unter NIV soll die Inhalation bevorzugt in den Spontanatemphasen durchgeführt werden.

E36 Patienten sollen vor Inhalation stets bis zur funktionalen Residualkapazität ausatmen.

E37 Bei Kindern unter 5 Jahren sollen für die Inhalation von Beta-2-Sympathomimetika oder Glucocorticosteroiden Treibgasdosieraerosole mit Spacer bevorzugt werden. Sobald als möglich sollte bei der Anwendung von Inhalationshilfen die Gesichtsmaske durch das Mundstück ersetzt werden.

gerätes müssen beachtet werden. Vor dem Hintergrund ständig neuer Einführungen sind Gründe für die fehlerhafte Handhabung des Inhalationsgerätes sowohl auf Seite des Verordners als auch auf Seite des Patienten zu suchen. Die Deutsche Atemwegsliga e.V. hat eine internetbasierte Aufklärungsplattform geschaffen. Verordner, Einweisungspersonal und Patienten können dort jederzeit Videos mit den notwendigen und korrekten Anleitungen zur Handhabung der jeweiligen Inhalationsgeräte abrufen [323, 324].

Bei der Auswahl des geeigneten Inhalationssystems sind die Fähigkeit zur Koordination von Sprühstoßauslösung und Inhalationsmanöver sowie die Höhe des Inspirationsflusses zu berücksichtigen [325 - 327]. Patientenpräferenzen sind ebenso in die Entscheidung miteinzubeziehen [328]. Für die Langzeittherapie soll möglichst nur ein Inhalationssystem (nur ein Typ eines Dosieraerosols oder eines Pulverinhalators) verordnet werden. Die Inhalationstechnik des Patienten soll regelmäßig und insbesondere bei unzureichender Kontrolle überprüft werden [329, 330]. Bei Wechsel eines Inhalationssystems soll die Neueinweisung des Patienten in die Handhabung erfolgen und die Notwendigkeit einer Dosisanpassung geprüft werden [331, 332].

Bei Auftreten von lokalen Nebenwirkungen infolge der Inhalation von Glucocorticosteroiden (Soorbefall, Dysphonie) sollte die oropharyngeale Deposition reduziert werden. Dies kann durch die Anwendung eines Dosieraerosols mit Spacer [333] 
oder durch die Inhalation des Glucocorticosteroids mittels Vernebler erreicht werden. Spacer erhöhen gering die pulmonale Deposition, sodass auch eine erhöhte Bioverfügbarkeit berücksichtigt werden sollte [334].

Neben der Kontrolle der korrekten Inhalationstechnik sollen Maßnahmen zur Verbesserung der Adhärenz geprüft werden. Dazu gehört eine Optimierung der Zahl der täglichen Inhalationen, ggf. sind Kombinationspräparate zu verwenden. Einbeziehung des Apothekenpersonals in die Schulung der Inhalationstechnik sowie die Verwendung auch internetbasierter Selbstmanagementprogramme können wirkungsvoll sein [324,330, 335-338].

Ein optimales Atemmanöver (nach tiefer Ausatmung) ist für die bronchiale Wirkstoffdeposition entscheidend:

- Dosieraerosol (mit/ohne Spacer): langsame tiefe Inspiration, anschließend Atempause (je kleiner die Teilchen, desto länger - bis zu 10 Sekunden)

- Pulverinhalator: rasche tiefe Inspiration, der Maximalfluss soll möglichst früh in der Inspiration erreicht werden, anschließend Atempause (siehe oben). Bei den Einzelkapselsystemen erfolgt die Dosisfreisetzung über einen längeren Zeitraum im Vergleich zu den Reservoirsystemen.

- Vernebler: langsame tiefe Inspiration [339]

- Cave: Im schweren Asthmaanfall sollten wegen eines unzureichenden inspiratorischen Flusses keine Pulverinhalatoren eingesetzt werden. Im Notfall kann die Inhalation mit einem Dosieraerosol mit Spacer oder mit einem Vernebler erfolgen.

Bei stationärer Behandlung des Asthmas soll die Inhalationstechnik überprüft und die Inhalationstherapie fortgeführt oder modifiziert werden. Ein unbegründetes Wechseln der Inhalationssysteme ist zu vermeiden. Auch auf Intensivstation ist die inhalative Therapie des Asthmas fortzuführen. Bei Patienten, die eine nicht invasive Beatmung durchführen, ist die Inhalation bevorzugt in den Spontanatemphasen durchzuführen [340]. Bei beatmeten Patienten kann die Inhalation mit Dosieraerosolen in Verbindung mit einem Spacer oder einem Vernebler erfolgen, die in den Inspirationsschenkel eingebracht werden [341].

Bei Kindern unter 5 Jahren sollten für die Langzeittherapie zur Inhalation Dosieraerosole mit Spacer bevorzugt werden. In der Akutphase sind Vernebler und Dosierareosole mit Spacer in ihrer Wirkung gleichwertig. Bei Anwendung von Verneblern oder Dosieraerosolen mit Spacer mit Gesichtsmaske sollte diese sobald wie möglich durch ein Mundstück ersetzt werden [342].

\section{Akuttherapie bei Asthma bronchiale}

Ein schwerer Asthmaanfall ist meist durch eine innerhalb von Minuten entstehende Atemwegsobstruktion charakterisiert. Klinisch berichten die Patienten über eine akut zunehmende Dyspnoe, Husten und Pfeifen über der Brust. Diese Symptome können einzeln oder in Kombination auftreten. Die Verschlechterung kann aber auch allmählich über Stunden entstehen. Die auslösenden Faktoren sind entweder nicht allergisch (z. B. kör-

\section{EMPFEHLUNGEN}

E38 Sauerstoff soll mit einer niedrigen Flussrate eingesetzt werden, um eine Sättigung von $93-95 \%$ bei Erwachsenen bzw. $>92 \%$ bei Kindern zu erreichen.

E39 Bei Exazerbation eines Asthmas sollen SABA als Mittel der ersten Wahl so früh wie möglich eingesetzt werden.

E40 Bei der schwereren Exazerbation sollen systemische Glucocorticosteroide in adäquater Dosis eingesetzt werden: Erwachsene $50 \mathrm{mg}$ Prednisolon-Äquivalent/Tag, Kinder 0,5-2 mg/kgKG/Tag, 0-2 Jahre maximal $20 \mathrm{mg} /$ Tag und 3-5 Jahre maximal $30 \mathrm{mg} /$ Tag.

E41 Ipratropiumbromid soll bei unzureichender Wirksamkeit der SABA zusätzlich eingesetzt werden.

E42 Inhalative Applikationsformen der Bronchodilatatoren (Dosieraerosol, Spacer, Vernebler) sollen gegenüber anderen Applikationsformen (i.v. oder s.c.) vorgezogen werden.

E43 Theophyllin als Infusion sollte nur nachgeordnet unter Beachtung des erhöhten Nebenwirkungsrisikos gegeben werden.

E44 Sedativa, Anxiolytika und Morphin sollen - falls erforderlich - nur in Intubationsbereitschaft verabreicht werden.

E45 Ipratropiumbromid (als Vernebler oder Dosieraerosol) soll zusätzlich zur Gabe von Beta-2-Sympathomimetika bei Patienten mit schwerem oder lebensbedrohlichem Asthmaanfall verabreicht werden.

E46 Magnesiumsulfat i.v. sollte beim akuten schweren Asthma mit schlechtem initialen Ansprechen und bei lebensbedrohlichem Asthma gegeben werden.

E47 Bei lebensbedrohlichem Asthma mit Verlegung des Patienten zur Intensivtherapie soll Intubationsbereitschaft bestehen. Es sollte frühzeitig eine Kontaktaufnahme mit einem in der Asthmabeatmung erfahrenen Zentrum aufgenommen werden.

E48 Die nicht invasive Beatmung (NIV) kann von einem mit dieser Methode erfahrenen Team bei unzureichendem Ansprechen der medikamentösen Therapie unter Beachtung der Kontraindikationen zusätzlich zur Standardtherapie durchgeführt werden, sie darf aber eine ggf. notwendige Intubation nicht verzögern.

S4 Sedativa und Anxiolytica wirken atemdepressiv und vermindern das Dyspnoe-Empfinden, ohne dass eine objektive Besserung bewiesen ist. 
liche Situation. Er macht, sofern er nicht im Anfangsstadium beherrschbar ist und eine Stabilisierung unter der Medikation ausbleibt, eine Krankenhauseinweisung und je nach Schwere auch eine intensivmedizinische Betreuung erforderlich. Im Notfall wird es kaum möglich sein, eine differenzierte Diagnostik durchzuführen. Deshalb ist in diesen Fall der klinische Befund für das weitere Procedere entscheidend. Im Hinblick auf ein effektives Management sollte die leichte und mittelschwere Asthmaexazerbation von der schweren Asthmaexazerbation grundsätzlich hinsichtlich Symptomatik und notwendige Therapie unterschieden werden [343].

\subsection{Leichte und mittelschwere Asthmaexazerbation beim Erwachsenen}

Symptome

- PEF $\geq 50$ \% PBW (persönlicher Bestwert)

- Sprechen normal

- Atemfrequenz $<25 /$ min

- Herzfrequenz $<110 / \mathrm{min}$

Initialtherapie

- zwei bis vier Hübe eines kurzwirksamen Beta-2-Sympathomimetikums (Dosieraerosol, ggf. mit Spacer), ggf. nach 10 - 15 Minuten wiederholen

- 20-25 mg Prednisolon (oral)

- Selbsthilfetechniken zur Atemerleichterung

\subsection{Schwere Asthmaexazerbation beim Erwachsenen}

Symptome

- $\mathrm{PEF}<50 \%$ PBW

- Sprech-Dyspnoe (Sprechen von lediglich Satzteilen oder Worten in einem Atemzug)

- Atemfrequenz $\geq 25 / \mathrm{min}$

- Herzfrequenz $\geq 110 / \mathrm{min}$

Initialtherapie

- 2-4l $\mathrm{O}_{2}$ /min über eine Nasensonde (Ziel: $\mathrm{SaO}_{2} 92 \%$ - 95\%)

- 2-4 Hübe eines kurzwirksamen Beta-2-Sympathomimetikums (Dosieraerosol, ggf. mit Spacer)

- $50-100 \mathrm{mg}$ Prednisolon-Äquivalent oral oder i.v.

- Ipratropiumbromid 0,5 mg durch Vernebler oder 4 Hübe $(=80 \mu \mathrm{g})$ aus einem Dosieraerosol

- Selbsthilfetechniken zur Atemerleichterung

\subsection{Lebensbedrohlicher Anfall} (Krankenhauseinweisung mit Notarztbegleitung)

- kein Atemgeräusch („stille Lunge“)

- atemerleichternde Stellung, Zyanose

- frustrane Atemarbeit/flache Atmung

- Erschöpfung, Konfusion, Bradykardie, Blutdruckabfall

- Peak flow $<33 \%$ PBW

- $\mathrm{SaO}_{2}<92 \%$

- $\mathrm{PaCO}_{2}$ normal oder $>45 \mathrm{mmHg}$
6.4 Therapiemaßnahmen während des Transportes zum Krankenhaus und im Krankenhaus bei unzureichendem Ansprechen auf die Initialtherapie

- 2-4l $\mathrm{O}_{2} /$ min über eine Nasensonde (Ziel: $\mathrm{SaO}_{2} 92 \%$ - 95\%) Cave: Hyperkapnie!

- 2 - 4 Hübe eines kurzwirksamen Beta-2-Sympathomimetikums, ggf. nach 10-15 Minuten wiederholen (max. alle 10 Minuten), oder 10 - 20 Tropfen in $1 \mathrm{ml} \mathrm{NaCl}$ über Vernebler alle 20 Minuten, ggf. zusätzlich IpratropiumbromidVerneblung (z. B. 0,5 mg bzw. 4 Hübe à $20 \mu \mathrm{g}$ aus Dosieraerosol alle 30 - 60 Minuten) [344,345].

- Beta-2-Sympathomimetika können auch parenteral verabreicht werden, z. B. Terbutalin 0,25-0,5 mg subkutan alle 4 Stunden, oder Reproterol 0,09 mg langsam intravenös (Wiederholung nach 10 Minuten möglich) bzw. 0,018-0,09 $\mathrm{mg} /$ Stunde ( = 5 Ampullen Reproterol auf $50 \mathrm{ml}$, Perfusor auf 2-10 $\mathrm{ml} /$ Stunde einstellen).

- 1 - $2 \mathrm{mg} / \mathrm{kg}$ Körpergewicht Prednisolon-Äquivalent (oral, intravenös oder bei Kleinkindern rektal), z. B. bei Erwachsenen 50 - $100 \mathrm{mg}$ intravenös alle 4-6 Stunden

- Magnesiumsulfat 2g/20 Minuten intravenös [346]

- Ausgleich einer metabolischen Azidose mit Bicarbonat bei $\mathrm{pH}<7,2$

- atemerleichternde Lagerung bzw. Körperposition

- Selbsthilfetechniken zur Atemerleichterung

Cave: Theophyllin (oder Aminophyllin) hat sehr wahrscheinlich keine zusätzliche bronchodilatatorische Wirkung im Vergleich zur oben beschriebenen Standardtherapie mit inhalativen Bronchodilatatoren und Glucocorticosteroiden. Es sollte daher nur bei Patienten mit lebensbedrohlichem Asthma und fehlender Besserung auf die initiale Therapie als Einzelfallentscheidung gegeben werden. Die TheophyllinDosis beträgt dann: initial $5 \mathrm{mg} / \mathrm{kg}$ Körpergewicht (KG) als Kurzinfusion; Erhaltungsdosis $0,5-0,7 \mathrm{mg} / \mathrm{kg} / \mathrm{KG} / \mathrm{h}$, bei vorheriger Theophyllin-Therapie zuerst Bestimmung der Serumkonzentration, dann Dosisanpassung (Cave: Intoxikation)

Cave: Bei der schweren Asthmaexazerbation kann die Kombination aus einem Anticholinergikum und einem Beta-2Sympathomimetikum besonders wirksam sein, während die alleinige Therapie mit einem Anticholinergikum unzureichend ist!

\subsection{Zur Behandlung des Asthmaanfalls sollten nicht eingesetzt bzw. möglichst vermieden werden:}

- Sedative und Anxiolytica wirken atemdepressiv und vermindern das Dyspnoe-Empfinden, ohne dass eine objektive Besserung bewiesen ist.

- Mukopharmaka sind kontraindiziert.

- Die Hydratation mit großen Flüssigvolumina führt zu einer kardialen Belastung.

- Antibiotika sind im Regelfall nicht indiziert und sollten nur eingesetzt werden, wenn der begründete Verdacht eines bakteriellen Auslösers der Exazerbation besteht. 


\subsection{Erhöhtes Mortalitätsrisiko im Asthmaanfall}

- Vorgeschichte eines beinahe letal verlaufenen Asthmaanfalls („Near-fatal Asthma“)

- notfallmäßige oder stationäre Behandlung des Asthmas im zurückliegenden Jahr

- vorherige Intubation und mechanische Beatmung wegen Asthma

- laufende systemische Glucocorticosteroid-Medikation oder kürzliches Absetzen einer systemischen Glucocorticosteroid-Therapie

- übermäßiger Einsatz von Beta-2-Sympathomimetika zur Symptomlinderung

- psychosoziale Probleme oder Negation von Asthma oder seines Schweregrades

- mangelnde Therapietreue in der Vergangenheit

\subsection{Indikation zur intensivmedizinischen Überwachung oder Behandlung}

Das Ansprechen auf die Initialtherapie sollte nach 30-60 min erfolgen. Dabei sollten jeweils der klinische Befund, die angegebenen Symptome, der Peak flow und die Sättigung (evtl. Blutgasanalyse) bestimmt werden. Eine mechanische Beatmung ist zu erwägen, bei

- Verschlechterung der PEF-Werte trotz Therapie

- persistierender oder zunehmender Hypoxämie

- Hyperkapnie

- fallendem arteriellen pH-Wert (Azidose)

- Erschöpfung

- Bewusstseinsstörung/Konfusion

- Koma oder Atemstillstand

\subsection{Kriterien zur Entlassung aus stationärer Behandlung}

- gutes Ansprechen auf die Therapie

- $\mathrm{FEV}_{1} \geq 70 \%$ des Sollwertes oder PEF $\geq 70 \%$ PBW über mindestens 60 Minuten

- keine angestrengte Atmung

- normaler klinischer Befund

\subsection{Nachsorge}

Beinahe letal verlaufene Asthmaanfälle in der Anamnese, eine schlechte Patientencompliance, der übermäßige Einsatz inhalativer Beta-2-Sympathomimetika, psychosoziale Probleme und Negierung des Asthmas bergen ein erhöhtes Risiko zum Wiederholungsfall. Schulungsmaßnahmen helfen, ein solches Risiko zu senken. Die Schulung des Patienten und ggf. auch enger Familienangehöriger (z. B. Ehepartner, Eltern betroffener Kinder) ist ein kontinuierlicher, vom Arzt begleiteter Prozess.

Ziel ist es, dem Patienten die pathophysiologischen Grundlagen des Asthmas und die Konsequenzen für die Diagnostik und Therapie näherzubringen. Er soll seine Erkrankung verstehen, die Maßnahmen zur Vorbeugung seines individuell angepassten Therapieplanes kennen und Selbsthilfemaßnahmen im Notfall beherrschen.
Dies schließt die Überprüfung der Inhalationstechnik, die Einweisung in einen Notfallbehandlungsplan, die Benutzung eines Peak-flow-Meters und ggf. auch die Therapieüberprüfung unter Berücksichtigung von Medikamentenunverträglichkeiten und kontraindizierten Substanzen wie z.B. Betablockern mit ein. Zudem sollten Erkrankungen identifiziert und therapiert werden, die mit einer Verschlimmerung asthmatischer Beschwerden einhergehen können:

- chronische Nasennebenhöhlenentzündungen

- gastroösophagealer Reflux

- Auslöser am Arbeitsplatz (Unfallrecht), z. B. exogen-allergisches Asthma im Back- und Friseurgewerbe.

\subsection{Therapie des schweren Asthmaanfalles beim Kind}

Bezüglich der Reihenfolge der Therapieoptionen gibt es keinen Unterschied, dennoch gibt es Besonderheiten:

1. Der Stellenwert von Beta-2-Sympathomimetika [347] und Ipratropiumbromid ist wie beim Erwachsenen einzuschätzen. Die Addition von letzterem zu ausreichend dosierter oder kontinuierlicher Beta-2-Sympathomimetika-Inhalation [348] bringt meist keine Verbesserung [349], aber besonders Kleinkinder reagieren in einzelnen Fällen besonders gut.

2. Der Stellenwert von Glucocorticosteroiden, Theophyllin und Magnesium ist beim Kind wie beim Erwachsenen einzustufen [350-354].

3. Da besonders bei Kleinkindern oft klinisch nicht zu entscheiden ist, ob ein schwerer Asthmaanfall oder eine Bronchiolitis vorliegt, ist bei mangelnder Besserung unter Beta-2Sympathomimetika besonders in dieser Altersstufe ein Versuch mit Adrenalin zur Inhalation gerechtfertigt, da hier Wirksamkeitsdaten vorliegen [355].

Die Fähigkeit zur Kooperation ist besonders beim Kleinkind nicht gegeben, was zu Schwierigkeiten in der Therapie führen kann. Zumindest bei Beatmung ist eine tiefe Sedierung und/ oder Muskelrelaxierung oft nicht zu umgehen. Das Anlegen einer Infusion etc. kann zur akuten respiratorischen Dekompensation durch Erregung führen, hierauf soll das Behandlungsteam vorbereitet sein. Oft lässt sich diese Schwierigkeit durch die Verwendung inhalierter, oraler oder rektaler Medikamentengaben (z.B. Glucocorticosteroide) zumindest in der kritischen initialen Phase vermeiden.

Die Inhalation ist für Kleinkinder besonders im schweren Anfall nicht einfach und wird manchmal abgewehrt oder nur insuffizient durchgeführt. Die Verwendung von Inhalierhilfen oder Spacern bei der Applikation von Dosieraerosolen ist daher auch im Anfall zwingend.

Eine Dauerinhalation (Feuchtinhalation mittels Verneblermaske und Sauerstoff) eines Beta-2-Sympathomimetikums hat sich als extrem effizient erwiesen [348, 356,357], es werden Serumspiegel wie bei i.v. Kurzinfusionen erreicht, das Legen einer Infusion kann dadurch zumindest initial vermieden werden. Dennoch ist auch eine Infusion von Beta-2-Sympathomimetika eine sinnvolle Option [347,354,358]. In besonders schweren Fällen könnte die Verwendung von Helium statt Sauerstoff als 
Treibgas im Inhaliersystem eine Symptomverbesserung bringen [359].

Für Kinder und Jugendliche gilt [360]:

Die kontinuierliche Inhalation von Salbutamol (0,5-1 mg/ $\mathrm{kg} / \mathrm{Std}$ ) ist die First-line-Bronchospasmolyse in der Intensivtherapie von Kindern mit schwersten Asthmaanfällen. Diese kann auch bereits auf dem Transport oder in der Notaufnahme angewendet werden, wenn der Asthmaanfall eine lebensbedrohliche Symptomatik bedingt. Wenn eine parenterale Therapie erforderlich ist, gelten folgende Dosisempfehlungen:

- In Deutschland ist Reproterol als Betamimetikum zur parenteralen Gabe bei schwerem Asthmaanfall ab dem 3. Lebensmonat zugelassen; die Dosierungsempfehlungen in der Fachinformation sind

- Kurzinfusion (Initial): $1 \mu \mathrm{g}$ Reproterolhydrochlorid/kg KG/ min über 10 Minuten

- Dauerinfusion: 0,2 $\mu \mathrm{g}$ Reproterolhydrochlorid/kg KG/min über 36-48 Stunden

- Magnesiumsulfat $25-50 \mathrm{mg} / \mathrm{kg} /$ Dosis $(\max 2 \mathrm{~g})$ über

20-30 min i. v., dann ggf. $15-25 \mathrm{mg} / \mathrm{kg} / \mathrm{Std}$.

- Cave Bradykardie

- Theophyllin: nach Loading dose 0,5 mg-1 mg/kg/Std., Spiegelkontrolle 30 min nach Ende der Loading dose, Spiegel $10-20 \mathrm{mg} / \mathrm{L}$

\subsection{Asthma und Beatmung}

\subsubsection{Invasive Beatmung}

Eine Intubation erfolgt nach klinischen Kriterien, die Zeichen der unzureichenden Wirkung der intensiven medikamentösen Therapie sind im Kapitel Therapie aufgeführt. Die Indikation zur mechanischen Beatmung richtet sich nach dem klinischen Verlauf und dem Ausmaß der Hypoxie und Hyperkapnie mit konsekutiver Azidose. Klinische Zeichen der unmittelbar bevorstehenden respiratorischen Erschöpfung sind paradoxe Atemexkursionen (Einziehung des Abdomens bei Inspiration, Vorwölbung bei Exspiration), Einziehungen über dem Jugulum, supraklavikulär und interkostal sowie Auftreten eines respiratorischen Alternans. Zur Intubation empfiehlt sich Ketamin bzw. S-Ketamin für die Sedation vor der Intubation, diese Substanzen stimulieren die Ausschüttung von Katecholaminen und haben wahrscheinlich einen direkten relaxierenden Effekt auf die Bronchialmuskulatur [361]. Die Beatmung ist wegen der hochgradigen Atemwegsobstruktion schwierig, da bei Aufrechterhaltung einer normalen alveolären Ventilation hohe Inspirationsdrücke notwendig sind. Diese führen häufig zu Hypotension und Barotrauma, vor allem besteht die Gefahr eines Pneumothorax. Hier wird die Strategie der „permissiven Hyperkapnie" als Standardtherapie empfohlen, was bedeutet, dass eine Hyperkapnie toleriert wird, um hohe Inspirationsdrücke zu vermeiden. Weiterhin soll bei der künstlichen Beatmung die Exspirationsdauer ausreichend lang sein, um eine Hyperinflation des Alveolarvolumens und damit eine Zunahme des Totraums zu vermeiden. Um den Atemwegswiderstand möglichst gering zu halten und damit auch die Exspiration zu erleichtern, sollte der Tubusdurchmesser möglichst groß gewählt werden.
Bei intubierten Patienten ist gerade bei einer schweren Dyskrinie die Bronchoskopie mit dem Absaugen der Sekretpfröpfe eine entscheidende Maßnahme zur Stabilisierung der Erkrankung. Sie ist auch diagnostisch von Bedeutung, um nicht seltene Fälle eines endobronchialen Tumors, eines Fremdkörpers oder einer Malformation zu übersehen. Nach vorheriger Gabe von Prednisolon $50 \mathrm{mg}$ i. v., topischer Lokalanästhesie und Vorinhalation über einen Vernebler mittels Sultanol oder Suprarenin ist die Bronchoskopie bei kurzer Untersuchungszeit durch einen erfahrenen Untersucher mit niedrigem Risiko möglich $[362,363]$. Bei nicht intubierten Patienten im Status asthmaticus auf der Intensivstation ist das Risiko der Bronchoskopie deutlich höher, dann müssen die Rahmenbedingungen einer raschen endobronchialen Intubation gegeben sein.

\subsubsection{Nicht invasive Beatmung (NIV)}

Die nicht invasive Beatmung führt bei akuter ventilatorischer Insuffizienz im Rahmen einer exazerbierten COPD im Vergleich zur Standardtherapie zu einer verringerten Mortalität, einer geringeren Intubationsrate und einer verkürzten Krankenhausdauer. Die Datenlage zur nicht invasiven Beatmung bei der akuten respiratorischen Insuffizienz durch eine schwere Asthmaexazerbation ist weniger evident. Trotz einiger prospektiver randomisierter kontrollierter Studien (RCT), die eine Verbesserung der Lungenfunktion sowie eine Verkürzung der stationären Aufenthalte bzw. eine geringere Rate an stationären Aufnahmen belegen, wird die nicht invasive Beatmung (NIV) aufgrund der geringen Fallzahlen in diesen Studien nicht uneingeschränkt, aber als Versuch bei Patienten mit schlechtem Ansprechen auf die medikamentöse Therapie empfohlen [364367]. Eine wichtige Voraussetzung für die Anwendung der NIV beim schweren Asthmaanfall ist neben der Auswahl der geeigneten Patienten eine ausreichende Erfahrung des behandelnden Teams in dieser Therapie. Klinische Erfolgskriterien der nicht invasiven Beatmung sind die Abnahme der Dyspnoe, eine Besserung der Vigilanz, eine Reduktion von Atem- sowie Herzfrequenz und ein ansteigender $\mathrm{pH}$-Wert.

Die nicht invasive Beatmung darf eine ggf. notwendige Intubation nicht verzögern. Innerhalb der ersten 2 Stunden soll sich der klinische Zustand des Patienten unter der nicht invasiven Beatmung gebessert haben. Bleibt die Besserung aus, verschlechtert sich der Patient oder treten Kontraindikationen auf, erfolgt die unverzügliche Intubation [368]. Kontraindikationen für die Durchführung einer nicht invasiven Beatmung sind

- Herz-Kreislauf- oder Atemstillstand

- mechanisches Hindernis der oberen Atemwege

- schwere Enzephalopathie

- erhöhtes Aspirationsrisiko/fehlende Möglichkeiten des Atemschutzes

- akute gastrointestinale Blutung

Auch bei Kindern ist die nicht invasive Beatmung sinnvoll mit denselben caveats wie bei Erwachsenen [369-372]. Die mangelnde Kooperationsfähigkeit macht die Anwendung beim Kleinkind schwieriger. Eine mögliche Einstellung bei Kindern für die NIV wäre: IPAP $10 \mathrm{~cm} \mathrm{H}_{2} \mathrm{O}$, EPAP $5 \mathrm{~cm} \mathrm{H}_{2} \mathrm{O}$. 
Auch bei Kindern wird die Beatmung mit eher niedrigen Atemfrequenzen, I:E-Verhältnissen von 1:3-1:5 und einem Atemminutenvolumen von $<115 \mathrm{ml} / \mathrm{kg}$ empfohlen, dabei kann das Tidalvolumen ca. $8-10 \mathrm{ml} / \mathrm{kg}$ sein und der inspiratorische Fluss 4-10 I/min [373]. Der gewählte Modus ist von lokalen Gegebenheiten abhängig. Die Verwendung eines volumenkontrollierten Modus bringt theoretische Vorteile, ebenso ein druckregulierter/volumenkontrollierter Modus. Eine permissive Hypoventilation wird angestrebt [373]. In extremen Situationen hat sich wegen der Kleinheit der Atemwege mit konsekutiver extremer Überblähung durch Auto-PEEP die exspiratorische manuelle Thoraxkompression beim beatmeten Kind bewährt.

\section{Nicht medikamentöse Therapie}

\subsection{Physiotherapie und Atemphysiotherapie}

Die Atemphysiotherapie führt zur Mobilisation und Kräftigung der muskulo-skelettalen Anteile der Atempumpe und zum allgemeinen Muskelaufbau [374-378]. Weiterhin kann mit der Atemphysiotherapie die dysfunktionelle Atmung (unphysiologische Atemmuster, z.B. Hyperventilation) auf ein physiologisches Atemmuster mit Verbesserung der Asthmakontrolle $[379,380]$ eingestellt werden. Hierbei werden die Mund- auf die Nasenatmung und die thorakalen auf bevorzugt abdominale Atemexkursionen umgestellt mit Kontrolle des Atemminutenvolumens bei chronischer Hyperventilation der Patienten. In ei- ner systematischen Übersicht über Studien mit Atemübungen und/oder Relaxationstraining unter Einschluss der Methoden von Buteyko und Papworth wurden Verbesserungen von Symptomen und Lebensqualität und/oder psychologischen Parametern berichtet, jedoch keine physiologischen Verbesserungen. Systematisches Atemtraining mit Biofeedbackmethoden kann jedoch auch zu zusätzlichen Verbesserungen der Lungenfunktion und Verringerung des Langzeitmedikationsbedarfs führen [379, 381]. Daher ergänzen diese Techniken sinnvoll die medikamentöse Asthmatherapie, können und sollen diese aber nicht ersetzen. Die Atemphysiotherapie wird auch zur individuellen Schulung eines Selbstmanagements im Asthmaanfall genutzt, um die Symptome Atemnot, Hustenreiz und Angst zu vermindern [382,383]. Atem- und Physiotherapieformen können in Einzelfällen sehr hilfreich sein und sollten als Ergänzung zum konventionellen Asthmamanagement betrachtet werden, insbesondere bei ängstlichen Patienten und denen, die einen inadäquat hohen Verbrauch an Notfallmedikation aufweisen [2].

\subsection{Alternative Methoden}

Komplementärmedizinische Therapieansätze Komplementäre oder alternative Therapieansätze haben sehr häufig den Nachteil, dass kontrollierte Effektivitätsstudien fehlen. Eine schlechte Studienlage bedeutet zwar nicht zwangsläufig, dass diese wirkungslos sind, aber Therapiemaßnahmen ohne belegte Wirkung können nicht oder nur bedingt empfohlen werden.

\section{EMPFEHLUNGEN}

E49 Jeder Patient mit Asthma und Indikation zu einer medikamentösen Langzeittherapie soll Zugang zu einer strukturierten Schulung erhalten.

E50 Der behandelnde Arzt sollte regelmäßig darauf hinweisen, dass der Patient geeignete Maßnahmen des körperlichen Trainings ergreift (z. B. Schulsport, Teilnahme an Lungensportgruppen). Ziel der Therapie ist es, eine altersgerechte uneingeschränkte Teilhabe an Sport zu ermöglichen. Insofern soll sportliche Aktivität empfohlen werden.

E51 Techniken der Atemphysiotherapie können mit dem Ziel der Reduktion von Atemnot, Hustenreiz und Angst sowie der Verbesserung des Selbstmanagements und der Lebensqualität eingesetzt werden.

E52 Bei adipösen Asthmapatienten soll eine Normalisierung des Körpergewichts empfohlen werden.

E53 Ärzte sollen allen rauchenden Patienten bzw. rauchenden Angehörigen zur Tabakabstinenz raten und sie auf die verfügbaren Entwöhnungshilfen hinweisen (medikamentöse Entwöhnungshilfen, Entwöhnungsprogramme).

S5 Die medikamentöse Therapie des Asthmas ist regelmäßig durch nichtmedikamentöse Therapiemaßnahmen (z. B. Tabakentwöhnung, Schulung, körperliches Training,
Atemphysiotherapie, Gewichtsreduktion bei adipösen Patienten) zu ergänzen.

S6 Eine strukturierte Patientenschulung führt zu einer bedeutsamen Verbesserung der Selbstmanagement-Fähigkeit mit besserer Symptomkontrolle, Verringerung der Zahl der Asthmaanfälle und Notfallsituationen, Verbesserung der Lebensqualität sowie Reduktion von Krankenhaus-, Arbeits- und Schulfehltagen. Eine günstige Kosten-/Nutzenrelation ist nachgewiesen.

S7 Körperliches Training kann zu einer Verringerung der Asthmasymptomatik, zur Besserung der Belastbarkeit und zur Verringerung der Morbidität beitragen.

S8 Rauchen verschlimmert das Asthma des Rauchers und der passiv rauchenden Erwachsenen, Kinder und Jugendlichen.

S9 Psychosoziale Beeinträchtigungen durch krankheitsbedingte Behinderungen im privaten sowie beruflichen Bereich sind bei Asthmapatienten häufig. Bei Kindern und Jugendlichen betrifft dies insbesondere die Auswirkungen auf die körperliche und seelische Entwicklung. Das familiäre und auch das soziale Umfeld können entscheidend die Akzeptanz der Diagnose und der notwendigen therapeutischen Maßnahmen beeinflussen. 
EMPFEHLUNGEN

E54 Die lonisierung (Air lonisers) der Luft soll in der Asthmatherapie nicht eingesetzt werden.

E55 Yoga und Buteyko-Atemtechniken können unterstützend zur Besserung der Asthmakontrolle eingesetzt werden.

E56 Hypnose und Relaxationstechniken können in Einzelfällen zur Besserung des subjektiven Befindens oder der Lungenfunktion beitragen.

S10 Akupunktur besitzt keinen nachgewiesenen Effekt auf die Asthmakontrolle.

S11 Manuelle Therapie einschließlich Massagen, Spinalmanipulationen und physisches Belastungstraining verbessern die Lungenfunktion nicht.

Akupunktur Innerhalb der Gruppe der alternativen Therapieansätze gibt es zur Akupunktur die meisten Studien. Metaanalysen belegen große methodische Probleme der Akupunkturstudien, die sich insbesondere auf die fehlende oder unzureichende Verblindung und die inkonsistenten Ergebnisse der verschiedenen Studien beziehen. Hepatitis C, die Entstehung von Pneumothoraces oder Verbrennungen sind in Abhängigkeit von der Art der verwendeten Nadeln und der Akupunkturtechnik als Nebenwirkungen beschrieben worden. Derzeit gibt es keine Empfehlung, die Akupunktur zur Asthmakontrolle einzusetzen.

Ionisierung (Air Ionisers) der Luft Auf dem Markt befinden sich Geräte zur Produktion negativ als auch positiv geladener Ionen. Es liegen zu beiden Techniken Studien vor [384-386], die jedoch keinen Effektivitätsnachweis in Hinblick auf die Verbesserung der Lungenfunktion, der Prognose oder der Exazerbationsrate erbrachten. Die lonisierung ist in der Asthmatherapie nicht empfohlen.

Hypnose und Relaxationstechniken Es gibt nur wenige kontrollierte Studien zur Effektivität von Hypnose- und Relaxationstechniken beim Asthma [387]. Allen gemeinsam sind ein inkonsistentes Studiendesign und methodische Probleme mit der Placebogruppe. Beide Techniken führen nicht zu einer Verbesserung der Lungenfunktion. Vor allem Relaxationstechniken gingen in Einzelstudien mit Verbesserungen der Lebensqualität, des psychischen Befindens oder der Lungenfunktion einher, wobei andere Studien keine entsprechenden Effekte erbrachten [388-390]. Daten zur Prognoseverbesserung fehlen.

Homöopathie Randomisierte kontrollierte Studien (RCT) konnten keinen Benefit der Homöopathie gegenüber der Standardmedikation belegen. Kritisiert wird, dass kein personalisierter homöopathischer Therapieansatz, ein wesentlicher Grundsatz der Homöopathie, getestet wurde. Die Homöopathie wird nicht zur Behandlung eines Asthmas empfohlen.

Kräutertherapie und traditionelle chinesische Medizin Die Vergleichbarkeit der publizierten Studien ist schwierig, da die Zusammensetzung der Testsubstanzen in RCTs stark variieren. Manche berichten über eine Verbesserung der bronchialen Hyperreaktivität (Ding Chuan Tang) und auch der Lungenfunktion.
Die Heterogenität der verfügbaren Studien erlaubt keine generalisierte Empfehlung für oder gegen einen solchen Therapieansatz. Kräutergemische können durchaus aktive und das Asthma positiv beeinflussende Bestandteile enthalten. Als Nebenwirkung sind Typ-I-Allergien zu beachten.

Sonstige alternative Therapieansätze Zu folgende Maßnahmen gibt es in Studien entweder keinen positiven Effektivitätsnachweis oder sie sind nicht in RCTs untersucht worden: Speläotherapie, Luftfeuchtigkeitskontrolle, Ernährungstherapie wie z. B. Fischöl, Fettsäuren, Mineralstoffsupplementation bzw. -restriktion, Vitamin-C-Gabe und Chiropraxis.

Als unterstützende Behandlung eines instabilen exogen-allergischen Asthmas mit einer Sensibilisierung gegenüber AeroAllergenen steht das Airsonett-Luftreinigungsgerät zur Verfügung. Es reduziert die Allergenlast in der Atemluft und führte nach einer einjährigen Anwendung zu einer signifikanten Verbesserung der Lebensqualität [391-393]. Daten einer multizentrischen Studie über Airsonett (temperature-controlled laminar airflow, TLA) zeigen eine Wirksamkeit bei Patienten mit mittelschwerem bis schwerem Asthma im Hinblick auf Zahl der Exazerbationen, z. B. bei Patienten mit schlechter Asthmakontrolle unter Stufe-4/5-Therapie kann der Einsatz eines Temperature-controlled Laminar Airflow die Asthmakontrolle verbessern [391].

\subsection{Rehabilitation}

Pneumologische Rehabilitation (PR) ist als ein umfassender multimodaler, multidisziplinärer Behandlungsprozess aufzufassen, der die meisten der dargestellten evidenzbasierten Einzelkomponenten nicht medikamentöser Therapien beinhaltet. Daher erscheint es zulässig zu postulieren, dass bei der nachgewiesenen Evidenz der einzelnen Behandlungskomponenten deren gleichzeitige Anwendung im Rahmen einer Rehabilitation entsprechende Effekte aufweisen wird. Bisher liegen zur Effektivität der Rehabilitation bei Asthma, auch aus Deutschland [394,395] und der Schweiz [396], eine Reihe von Beobachtungsstudien vor. Die Ergebnisse einer großen deutschen RCT zur Rehabilitation beim Asthma bronchiale werden zum Jahreswechsel 2017/18 erwartet [397].

Einschränkend ist zu beachten, dass eine multimodale Rehabilitation hinsichtlich Inhalt, Intensität, Qualität i.S. zugrunde liegender Expertise nicht eindeutig definiert ist. Zudem ist zu bedenken, dass die studienmäßige Testung von Einzelkomponenten nicht unter Alltagsbedingungen, sondern mit hoher Motivation und Expertise der Durchführenden für die Intervention getestet wurden. Somit ist ein ausreichendes Maß an Durchführungsqualität seitens der Reha-Anbieter zum Erreichen relevanter Effekte zwingende Voraussetzung [398].

Pneumologische Rehabilitation bei Asthma soll Patienten dabei unterstützen, ihre bestmögliche individuelle physische und psychische Integrität zu erhalten oder wieder zu erlangen [399-402]. Es ist eine normale oder bestmögliche soziale Teilhabe in Schule, Beruf und Freizeit anzustreben. Dies zum einen für den Patienten selbst, bei Kindern und Jugendlichen aber auch für deren Familien. Die Rehabilitation ist somit als wesentlicher Bestandteil einer am langfristigen Erfolg orientierten 


\section{EMPFEHLUNGEN}

E57 Patienten mit nur partiell kontrolliertem oder unkontrolliertem Asthma soll eine pneumologische Rehabilitation angeboten werden, um normale Aktivitäten bzw. Teilhabe am beruflichen und privaten Leben zu ermöglichen.

E58 Bei stationär behandelten Asthmaexazerbationen soll vor Entlassung aus dem Akutkrankenhaus die Indikation zur Anschlussheilbehandlung geprüft werden.

E59 Nach rezidivierenden Asthmaexazerbationen soll die Indikation zu rehabilitativen Maßnahmen bei jedem Patienten geprüft und ggf. organisatorisch vorbereitet werden.

E60 Bei Jugendlichen, die vor dem Eintritt in die berufliche Ausbildung stehen, sollte während der Rehabilitation eine Berufsberatung erfolgen.

E61 Jedem Patienten sollen individuelle Empfehlungen zur Nachbehandlung und zur Verstetigung des Behandlungserfolges gegeben werden.

S12 Wesentliche Komponenten der Rehabilitation beim Asthma sind: personalisierte Asthmadiagnostik inklusive Allergiediagnostik, Überprüfung von Allergenexposition, beruflichen und privaten Triggerfaktoren und Optimierung der medikamentösen und nicht medikamentösen Therapie. Dazu gehören insbesondere strukturierte Tabakentwöhnungsprogramme, Patientenschulung, Trainingstherapie, Physiotherapie/Atemphysiotherapie, Ergotherapie, Hilfsmittelberatung, Sozial- und Berufsberatung, psychologische und ggf. psychotherapeutische Hilfen, Ernährungsberatung, sozialmedizinische Beratung.

S13 Der multimodale Ansatz der Rehabilitation bewirkt mehr als die Summe der einzelnen, aufgezählten nicht medikamentösen Therapieverfahren.

Asthmatherapie zu verstehen. Während für Erwachsene definierte Strukturen für eine ambulante Rehabilitation teilweise vorhanden sind, existieren diese für Kinder und Jugendliche nicht und sind wegen der Abhängigkeit von der elterlichen Unterstützung auch kaum flächendeckend umsetzbar.
Die Rehabilitation ist in den jeweiligen Gesundheitssystemen durch Sozialgesetzgebung hinsichtlich Anspruchsberechtigungen und Kostenträgerzuständigkeiten geregelt. Für den Bereich der Bundesrepublik Deutschland ist die Trägerstruktur für erwachsene Patienten in der $>$ Tab. 12 dargestellt. Bei Kindern und Jugendlichen besteht eine gleichrangige Zuständigkeit der Rentenversicherung und der Krankenversicherung (§40 SGB V).

Zur Erreichung der durch pneumologische Rehabilitation möglichen Behandlungserfolge ist es notwendig, seitens der Antragsteller wie auch der Kostenträger auf die notwendige Gesamtexpertise der Rehabilitationsleistungserbringer zu achten.

Bezüglich der Kostenträger bei der Rehabilitation von Kindern und Jugendlichen besteht hier grundsätzlich eine gleichrangige Zuständigkeit zwischen der GKV und der Deutschen Rentenversicherung (DRV). Faktisch werden jedoch rund $90 \%$ der Rehabilitationsmaßnahmen von der DRV übernommen. Rehabilitation ist keine Mutter(Vater)-Kind-Kur. Hier werden andere Ziele angestrebt und andere Qualitätskriterien angelegt. Im Fokus der Mutter(Vater)-Kind-Kur stehen die Mütter(Väter), bei Rehabilitation geht es um die Erkrankung bzw. soziale Teilhabe des chronisch kranken Kindes oder des Jugendlichen. Im Vordergrund stehen hier also nicht Klimaaspekte, sondern medizinische und/oder therapeutische Inhalte.

Das im Dezember 2016 in Kraft getretene Flexirentengesetz eröffnet grundsätzlich neue Dimensionen der Vernetzung zwischen ambulanten, klinischen und rehabilitativen Versorgungsstrukturen in Deutschland. Es stellt einen Paradigmenwechsel im Bereich der pädiatrischen Rehabilitation dar. Insbesondere sind hier zu nennen:

- Kinder- und Jugendrehabilitation wird Pflichtleistung bei der DRV.

- Kinder- und Jugendrehabilitation wird eigenständig gesetzlich ausgestaltet.

- Rehabilitation kann sowohl stationär als auch ambulant erbracht werden.

- Die bisherige Begrenzung der Ausgaben entfällt.

- Indikationsbeschränkungen werden aufgehoben.

- Neben der späteren Erwerbsfähigkeit wird die Schul- und Ausbildungsfähigkeit als Ziel klar benannt.

- Tab.12 Übersicht der Trägerstruktur der medizinischen Rehabilitation in Deutschland.

\begin{tabular}{|c|c|c|}
\hline $\begin{array}{l}\text { Gesetzliche Rentenversicherung (GRV) } \\
\text { z. B. Deutsche Rentenversicherung }\end{array}$ & $\begin{array}{l}\text { Gesetzliche Krankenversicherung (GKV) } \\
\text { z. B. AOK, Ersatzkrankenkassen }\end{array}$ & $\begin{array}{l}\text { Gesetzliche Unfallversicherung (GUV) } \\
\text { z. B. Berufsgenossenschaften }\end{array}$ \\
\hline $\begin{array}{l}\text { Leistungen zur medizinischen Rehabilitation } \\
\text { können einem Versicherten oder Rentner } \\
\text { gewährt werden, wenn ( } \$ 10 \text { SGB VI) } \\
\text { " die erhebliche Gefährdung der Erwerbs- } \\
\text { tätigkeit abgewendet, } \\
\text { " die bereits geminderte Erwerbstätigkeit } \\
\text { wesentlich gebessert oder wiederherge- } \\
\text { stellt oder deren wesentliche Verschlech- } \\
\text { terung abgewendet werden kann. }\end{array}$ & $\begin{array}{l}\text { Leistungen zur medizinischen Rehabilitation } \\
\text { werden gewährt, ( } \$ \S 27,40 \text { SGB V) um } \\
\text { " drohender Behinderung, } \\
\text { - Pflegebedürftigkeit vorzubeugen oder } \\
\text { sie nach Eintritt } \\
\text { " zu beseitigen, zu bessern, eine Verschlim- } \\
\text { merung zu verhüten. }\end{array}$ & $\begin{array}{l}\text { Leistungen zur medizinischen Rehabilitation } \\
\text { werden erbracht ( } \$ 7 \text { SGB VII) } \\
\text { - aufgrund eines Arbeitsunfalls oder } \\
\text { - nach Eintritt einer anerkannten Berufs- } \\
\text { krankheit. } \\
\text { - Die Leistungen sollen den Gesundheits- } \\
\text { schaden (§26 SGB VII) } \\
\text { - beseitigen, bessern, eine Verschlimmerung } \\
\text { verhüten oder die Folgen mildern. }\end{array}$ \\
\hline
\end{tabular}


- Eine Begleitperson wird, altersunabhängig, genehmigt, wenn sie zur Durchführung oder für den Erfolg notwendig ist.

- Erstmalig kann die DRV Leistungen zur Nachsorge finanzieren, wenn sie zur Sicherung des Erfolgs einer durchgeführten Rehabilitation erforderlich sind.

- Die Vierjahresfrist entfällt bei Kindern und Jugendlichen.

Bei der Antragstellung sind die Asthma-bedingten Krankheitsfolgen im Sinne der „International Classification of Functioning, ICF" zu formulieren [403]. Dabei spielen die gesamten bio-psycho-sozialen Aspekte der Erkrankung eine größere Rolle als die reine Darstellung von Messwerten. Im Einzelnen sind die krankheitsbedingten Fähigkeitsstörungen sowie die eingeschränkte Teilhabe am schulischen, beruflichen und gesellschaftlichen Leben einschließlich des vorhandenen Rehabilitationspotenzials darzustellen. Die pneumologische Rehabilitation zielt nicht nur auf die Beseitigung oder Kompensation körperlicher, sondern auch psychischer und sozialer Krankheitsfolgen ab bzw. soll deren Verschlimmerung verhindern.

Das systematische Erfassen dieser bio-psycho-sozialen Beeinträchtigungen ist Voraussetzung zum Erarbeiten individueller Rehabilitationsziele. Hierbei kommt der Sicherung oder Wiederherstellung einer stabilen Alltagstauglichkeit besondere Bedeutung zu. Bei Erwachsenen stellt die Erwerbsfähigkeit einen wesentlichen Aspekt dar [404]. Bei Kindern und Jugendlichen ist es das Ziel, eine normale schulische Laufbahn und hierdurch eine bestmögliche Ausbildungs- und Erwerbsfähigkeit wieder herzustellen oder zu sichern. Der Unterricht dient auch dazu, Schulleistungsstörungen aufzudecken. Er liefert Hinweise auf weiterführende Maßnahmen. Schule ist Alltag für Kinder und Jugendliche und ist entsprechend einer Arbeitserprobung als "Belastungserprobung" während der Rehabilitation zu werten. In diesem Zusammenhang gibt sie Anregungen für die Nachbetreuung. Insgesamt ist eine normale soziale Teilhabe anzustreben. Dieses Therapieziel gilt auch für nicht Erwerbstätige und Rentner in gleichem Maße.

Um diesen multimodalen Therapieansatz realisieren zu können, ist ein multiprofessionelles Reha-Team notwendig, bestehend aus Ärzten, Psychologen, Pflegepersonal, Sozialpädagogen, Ernährungsberatern sowie Physio-, Ergo- und Sporttherapeuten. In der Kinder- und Jugendlichen-Rehabilitation ist eine adäquate Beschulung zu gewährleisten. Jedem Mitglied dieses Teams müssen die individuell vereinbarten und mit den Patienten abgestimmten Rehabilitationsziele bekannt sein. Jeder Reha-Prozess soll durch regelmäßige Teambesprechungen begleitet werden, um notwendige Anpassungen oder Korrekturen realisieren zu können.

Unter den Einzelkomponenten spielt die strukturierte Schulung nach evaluierten und veröffentlichten Modellen (z.B. AG Asthmaschulung im Kindes- und Jugendalter) eine herausragende Rolle, um die Eigenkompetenz der Patienten bestmöglich zu fördern. Dabei soll insbesondere bei Kindern das soziale Umfeld, hier v. a. die Eltern unbedingt in den Schulungsprozess einbezogen werden $[405,406]$. Bei Kindern im Vorschulalter soll eine spezielle Schulung der Eltern durchgeführt werden. Diese hat sich in einer breit angelegten Multicenterstudie als ef- fektiv erwiesen [407]. Da sich regelmäßige Nachschulungen als effektiv erwiesen haben, sollten die Patienten auf diese Möglichkeit hingewiesen werden. Bei Kindern und Jugendlichen sind dabei für diese Altersgruppen evaluierte Modelle gemäß Qualitätshandbuch der AG Asthmaschulung (4. Auflage 2013) einzusetzen [408].

Alle obengenannten Maßnahmen müssen qualitätsgesichert und auf hohem Niveau erfolgen. Entsprechende Kriterien zur Qualitätskontrolle werden insbesondere von der DRV regelhaft eingesetzt (z. B. evidenzbasierte Therapiemodule). Dies impliziert auch, dass eine erfolgreiche Rehabilitation einen hohen fachlichen und diagnostischen Standard in allen Professionen erfordert und dass sogenannten „Klimamaßnahmen“ alleine keinesfalls ausreichend sind.

Prinzipiell sind Maßnahmen zur pneumologischen Rehabilitation ambulant wie auch stationär möglich. Im deutschsprachigen Raum stehen allerdings im internationalen Vergleich weit weniger, teils, insbesondere bei Kindern und Jugendlichen, noch gar keine adäquaten ambulanten Reha-Strukturen zur Verfügung.

Ambulante Verfahren sind jedoch nicht indiziert z. B. bei instabilem Asthma, komplexer Komorbidität und Drogenabhängigkeit. Die ambulanten Strukturen können sowohl auf eine stationäre Rehabilitation vorbereiten als auch danach die Behandlungskette im Verlauf durch erneute Asthmaschulungen vervollständigen. Die Anfahrtszeit für eine ambulante Rehabilitation sollte 45-60 Minuten nicht überschreiten.

Die Indikation für eine stationäre Rehabilitation ist dann gegeben, wenn trotz Ausschöpfung der verfügbaren ambulanten Therapiemöglichkeiten ein adäquates Asthmamanagement und eine alterstypische Lebensqualität im Alltagsleben nicht erzielbar sind. Dies ist gegeben bei:

- persistierenden asthmatischen Beschwerden bzw.

Einschränkungen der Lungenfunktion

- krankheitsbedingter Gefährdung der Erwerbstätigkeit (E) bzw. eines geeigneten und angemessenen Schulabschlusses (K) bzw. einer Berufsausbildung

- drohender Pflege- und Hilfsbedürftigkeit

- Notwendigkeit von rehabilitationsspezifischen, nicht medikamentösen Therapieverfahren, wenn diese nicht ambulant im erforderlichen Ausmaß erfolgen können

- Erfassung des Schweregrades und der Ausprägung der Erkrankung unter alltagsnahen Bedingungen

- Vorliegen einer Schädigung einer Körperfunktion und/oder Körperstruktur (z. B. eingeschränkte körperliche Belastbarkeit)

- Vorliegen einer Beeinträchtigung (verminderte Lebensqualität, Störung der sozialen Integration, drohende berufliche Beeinträchtigung z. B. wegen bestimmter Allergene)

- Notwendigkeit einer adäquaten medikamentösen Therapieeinstellung unter klinischer Kontrolle bei ganztägiger Beobachtungsmöglichkeit in unterschiedlichsten Situationen

- therapielimitierenden Faktoren im Bereich des psychosozialen Umfeldes (familiäre Probleme, Verhaltens- oder Aufmerksamkeitsstörungen, emotionale Störungen)

- fehlendem therapeutischen Konsens zwischen Ärzten, Kindern und deren Familien 
- ungenügender Krankheitseinsicht und Krankheitsakzeptanz

- fehlender Compliance durch mangelhaftes Krankheitsverständnis, fehlender Schulung

- häufigen Schulfehlzeiten und daraus resultierender negativer Leistungsspirale

- Notwendigkeit des Erlernens spezieller Therapien oder Techniken

- Problemen im Bereich des Selbstwertgefühls und der sozialen Kompetenz

Wesentliche Komponenten der Rehabilitation beim Asthma bronchiale sind:

- Diagnosespezifizierung (u.a. Rehabilitationsdiagnostik auf der Ebene von Aktivität und Partizipation, Erfassung der somatischen und psychischen Komorbidität sowie der Lebensqualität)

- Überprüfung des Schweregrades und ggf. Optimierung der medikamentösen Therapie

- Patientenschulungen und Verhaltenstraining

- Atemphysiotherapie/Krankengymnastik

- medizinische Trainingstherapie

- Ergotherapie inklusive Hilfsmittelberatung

- Sozial- und Berufsberatung

- strukturierte Tabakentwöhnung

- psychologische und ggf. psychotherapeutische Hilfen

- Karenzempfehlungen bzgl. Allergenen, Schadstoffen, Trigger- und Risikofaktoren

- sozialmedizinische Beurteilung

- ggf. grundlegende psychologische Testung und Beurteilung

- Transfer von krankheitsspezifischem und sozialem Verhalten in den Alltag über die Sozialpädagogik unter Einbeziehung der Eltern

- Vertiefung und Einübung der Schulungsinhalte im Rahmen des Rehabilitationsalltags, wenn möglich unter Einbeziehung der Eltern

Effekte der pneumologischen Rehabilitation beim Asthma bronchiale können sein [394, 409, 410]:

- Reduktion der klinischen Leitsymptome wie Atemnot, Husten, Auswurf

- Verbesserung der allgemeinen und krankheitsspezifischen Lebensqualität

- Besserung der Asthmakontrolle

- Steigerung der körperlichen Leistungsfähigkeit

- Verbesserung der beruflichen und privaten Partizipationsfähigkeit

- Abnahme des akutmedizinischen Ressourcenverbrauchs (Krankenhaustage, Notfallbehandlungen)

- Verringerung von psychosozialen und familiären Auswirkungen

- Wiedereingliederung in den schulischen Alltag

- Beratung in Hinblick auf eine spätere Ausbildungs- und Berufswahl

- Heranführen des Kindes/Jugendlichen an eine normale sportliche Aktivität
Nachgewiesen wurde bei Kindern und Jugendlichen ein positiver Effekt auf die gesundheitsbezogene Lebensqualität durch eine stationäre Rehabilitation wegen Asthma [411,412]. Auch bei Erwachsenen wurde ein positiver Effekt pneumologischer Rehabilitation auf die Lebensqualität gezeigt [413-415].

Die Einbindung von stationären Rehabilitationsmaßnahmen unter Berücksichtigung der Eigenverantwortung des Patienten in suffiziente Vor- und Nachsorgeprogramme (z. B. ambulanter Lungensport, ambulante Schulungen/Nachschulungen) ist notwendig. Hier existieren einerseits Nachschulungsstrukturen seitens der Arbeitsgemeinschaft Asthmaschulung im Kindesund Jugendalter [416], andererseits auch im DMP anerkannte und evaluierte internetbasierte Nachschulungsprogramme für Kinder und Jugendliche (z.B. my-air.tv). Flächendeckend und kostenträgerübergreifend sind solche Strukturen jedoch noch nicht vorhanden.

\subsection{Patientenschulung}

Eine strukturierte und bei Kindern und Jugendlichen die Familie einbeziehende Patientenschulung führt zu einer klinisch bedeutsamen Verbesserung der Selbstmanagementfähigkeiten mit besserer Symptomkontrolle, Verringerung der Zahl von Asthmaanfällen und Notfallsituationen, Verbesserung der Lebensqualität sowie Reduktion von Krankenhaus-, Arbeitsunfähigkeit- bzw. Schulfehltagen [417-419]. Eine günstige Kosten-Nutzen-Relation ist nachgewiesen.

Diese positiven Effekte sind jedoch nur für Schulungsprogramme gesichert, bei denen Inhalte des Selbstmanagements wie Selbstkontrolle (Symptomatik und Peak-flow-Verlauf) und selbstständige Anpassung der Medikation (Therapie und Notfallplan) vermittelt werden, nicht hingegen für Programme mit reiner Wissensvermittlung ohne verhaltensmodifizierende Ansätze $[418,420]$.

Jeder Patient mit Asthma soll daher zeitnah nach der Diagnose Zugang zu einem strukturierten, evaluierten, zielgruppenspezifischen und qualitätsgesicherten Schulungsprogramm erhalten. Bei der Schulung von Kindern und Jugendlichen sind die Eltern regelhaft einzubeziehen. Regelmäßige Nachschulungen (nach ca. 2 Jahren) sind empfehlenswert. Patientenschulung soll eine aktive Teilnahme des Patienten (bei Kindern und Jugendlichen auch deren Familien) an der Bewältigung seiner chronischen Krankheit durch Überwachen der Symptomatik und adäquate Selbstanpassung der Therapie an den jeweiligen Schweregrad der Erkrankung ermöglichen. Der Erkrankte soll in die Lage versetzt werden, seine Symptome zu erkennen, zu bewerten und zu kontrollieren sowie Verschlechterungen durch eine rechtzeitige Therapieanpassung zu beherrschen. Er sollte seine Medikamente (Controller, Reliever) mit Wirkungen und unerwünschten Effekten kennen sowie in der Lage sein, diese korrekt anzuwenden (Training der Inhalationstechnik) und Selbsthilfemaßnahmen bei einem Asthmaanfall einzusetzen. Dazu gehören auch ein schriftlicher Therapie- und Notfallplan einschließlich der Bereitstellung der dafür erforderlichen Notfallmedikation sowie geeignete Instruktionen zu atemphysiotherapeutischen Selbsthilfemaßnahmen (atemerleichternde Körperstellungen, Lippenbremse) [421]. 


\subsection{Körperliches Training}

Bei Patienten mit Asthma führt moderates körperliches Training wie auch in der Allgemeinbevölkerung zu wesentlichen gesundheitlichen Verbesserungen mit reduziertem kardiovaskulären Risiko und verbesserter Lebensqualität [422,423]. 2 RCTs belegen eine Verbesserung von Symptomen und der Lebensqualität durch körperliches Training bei erwachsenen Asthmatikern [424,425]. Zudem ging Training mit einer verbesserten Asthmakontrolle einher [426]. Bei Jugendlichen wird Schwimmen gut toleriert und führt zu einer Verbesserung der Lungenfunktion und der kardiovaskulären Belastbarkeit [427]. Daher sollte der behandelnde Arzt darauf hinwirken, dass der Patient geeignete Maßnahmen des körperlichen Trainings ergreift (z. B. die Teilnahme an ambulanten Lungensportgruppen) [428]. Ist dies unter den häuslichen Bedingungen nicht möglich, sollte die Zuweisung in ein ambulantes oder stationäres Rehabilitationsprogramm erwogen werden.

Art und Umfang des körperlichen Trainings sollten einmal jährlich überprüft werden. Insbesondere sollte darauf hingewirkt werden, dass Schulkinder mit Asthma unter Berücksichtigung der individuellen und aktuellen Leistungsfähigkeit regelmäßig am Schulsport teilnehmen.

\subsection{Tabakentwöhnung}

Tabakrauch verschlimmert das Asthma des Rauchers [429] und der passivrauchenden Erwachsenen, Kinder [430] und Jugendlichen. Bei Patienten mit Asthma hat Zigarettenrauchen multiple negative Effekte zusätzlich zu den bekannten Effekten wie dem erhöhten Risiko für Lungenkrebs, COPD und kardiovaskuläre Erkrankungen. Zigarettenrauchen während der Schwangerschaft führt bei den Kindern zu einem Anstieg des Risikos der späteren Entwicklung von Asthma bronchiale und Atemwegserkrankungen.

Bei Patienten mit Asthma (Kinder und Erwachsene) führt passives Tabakrauchen zu einem erhöhten Risiko von Krankenhausaufenthalten und schlechter Asthmakontrolle. Aktives Rauchen ist mit einem ansteigenden Risiko einer schlechten Asthmakontrolle, häufigeren Krankenhausaufenthalten und zumindest in einigen Studien mit einer erhöhten Mortalität durch Asthma verbunden. Aktives Rauchen fördert die Verschlechterung der Lungenfunktion und kann zur COPD führen. Es reduziert den Effekt inhalativer und oraler Glucocorticosteroide [72]. Nach Aufgabe des Rauchens kommt es zu einer Verbesserung der Lungenfunktion und Verminderung der Atemwegsentzündung [431]. Eine Reduktion der passiven Rauchexposition führt zu einer Verbesserung der Asthmakontrolle und reduziert die Krankenhausaufenthalte von Erwachsenen und Kindern [432].

Die Dokumentation der Rauchgewohnheiten und der personenbezogene Rat zur Abstinenz gehören zu den Elementarpflichten des behandelnden Arztes. Für änderungsbereite Raucher sollten nichtmedikamentöse und medikamentöse Hilfen zur Raucherentwöhnung angeboten werden. Bzgl. der Methoden der Tabakentwöhnung wird auf die Leitlinie „Tabakentwöhnung" verwiesen [433].

\subsection{Die Kontrolle des Körpergewichtes bei adipösen Patienten}

Adipositas erschwert bei Patienten mit Asthma die Asthmakontrolle [434-436] und vermindert den Effekt der inhalativen Glucocorticosteroide [437]. Eine Gewichtsabnahme verbessert die Asthmakontrolle, die Lungenfunktion sowie den Allgemeinzustand, außerdem wird die Anzahl der Medikamente reduziert [438, 439]. Ganz entscheidende Verbesserungen wurden nach bariatrischen Operationen beschrieben [440,441]. Selbst eine nur 5-10\%ige Gewichtsabnahme mit Diät, mit oder ohne Training, führt zu einer Verbesserung der Asthmakontrolle und der Lebensqualität [442].

\subsection{Bronchiale Thermoplastie}

Unter bronchialer Thermoplastie versteht man eine lokale Radiofrequenz-Puls-Behandlung der Atemwege während dreier separater Bronchoskopien [443-445]. Die Behandlung ist mit einem großen Placeboeffekt assoziiert [443]. Bei Patienten unter Therapie mit hochdosierter medikamentöser Behandlung mit ICS/LABA ist die Behandlung mit einem Anstieg der Asthmaexazerbationen während der ersten 3 Monate verbunden, anschließend kommt es zu einem Abfall der Exazerbationen, jedoch ohne positiven Effekt auf die Lungenfunktion oder die Asthmasymptomatik im Vergleich zur Placebogruppe [443]. Allerdings findet sich in einigen Kohorten eine Reduktion der Exazerbationsfrequenz im Vergleich zum Status vor der Thermoplastie [446]. Zur sicheren Beurteilung sind Langzeitverläufe mit höherer Patientenanzahl notwendig, um Effektivität und Sicherheit der Methode zu beurteilen. Die Anzahl der Studien ist gering, Patienten mit chronischer Sinusitis, häufigen Atemwegsinfektionen und einer $\mathrm{FEV}_{1}$ von $<60 \%$ wurden in den Studien ausgeschlossen. Es wird empfohlen, die Methode nur im Rahmen von Studien oder zumindest Registern anzuwenden [128].

\section{Asthma und Komorbiditäten}

Die Erkrankung Asthma ist mit mehreren Komorbiditäten vergesellschaftet. Im Folgenden wird auf die einzelnen Entitäten eingegangen. Ausführungen zum gemeinsamen Auftreten von Erkrankungen der oberen Atemwege (vor allem Rhinitis und Sinusitis), deren Diagnostik und Therapie finden sich im Kapitel Asthma und Rhinitis. Psychiatrische Erkrankungen (vor allem Depression und Angststörungen) und Asthma sind in epidemiologischen Studien miteinander assoziiert. Weitere Ausführungen zu diesem Thema finden sich im Kapitel Asthma und Psyche.

\subsection{Asthma und Reflux}

In mehreren großen epidemiologischen Studien konnte gezeigt werden, dass Asthma und gastroösophagealer Reflux miteinander assoziiert sind. Die Prävalenz von Reflux ist bei asthmatischen Patienten etwa 1,5 bis 2 Mal höher als bei nicht asthmatischen Patienten [447-452]. Daher sollte bei Patienten mit Asthma auch an eine Reflux-Erkrankung gedacht und diese diagnostiziert werden. Eine symptomatische Reflux-Erkrankung 


\section{EMPFEHLUNGEN}

E62 Bei Patienten mit Asthma sollte an eine Refluxerkrankung gedacht und diese diagnostiziert werden. Nur eine symptomatische Refluxerkrankung soll behandelt werden.

E63 Asthma und obstruktives Schlafapnoe-Syndrom (OSAS) sind in epidemiologischen Studien miteinander assoziiert. Bei Patienten mit Asthma sollte die Schlafapnoe leitliniengerecht behandelt werden.

E64 Adipositas und Asthma sind in epidemiologischen Studien miteinander assoziiert. Die Therapie einer begleitenden Adipositas soll mit verschiedenen Modalitäten durchgeführt werden, um eine Verbesserung der Asthmakontrolle zu erreichen.

E65 Bei Patienten mit Asthma und bei Patienten mit chronischer Rhinosinusitis sollte immer nach der jeweiligen Komorbidität gesucht werden.

E66 Im Rahmen der Primärprävention des Asthmas soll bei Kindern die Indikation zur Immuntherapie geprüft werden.

E67 In Anamnese und Behandlungskonzept des Asthmas sollten psychische Komorbiditäten und psychosoziale Problemkonstellationen berücksichtigt werden und ggf. psychologische bzw. psychotherapeutische Hilfen angeboten werden.

S14 Dysfunktionale Atmung (z.B. Vocal cord dysfunction, VCD) und Asthma sind in epidemiologischen Studien miteinander assoziiert bzw. schwierig gegeneinander abzugrenzen. Eine dysfunktionale Atmung ist als möglicher aggravierender Faktor bei schwierigem Asthma bronchiale zu berücksichtigen.

S15 Ein niedriger Vitamin-D-Spiegel und Asthma, insbesondere schweres Asthma, sind in epidemiologischen Studien miteinander assoziiert.

S16 Erkrankungen der oberen Atemwege sind ein Risikofaktor für die Entstehung eines Asthmas bzw. können den Verlauf eines Asthmas negativ beeinflussen. Die Therapie einer allergischen Rhinitis oder eines Asthmas kann potenziell die jeweils andere koexistente Erkrankung positiv beeinflussen.

sollte adäquat behandelt werden. Die Behandlung eines asymptomatischen Refluxes kann nach evidenzbasierten Kriterien nicht empfohlen werden. Nachdem kleinere Studien inkonsistente positive Effekte gezeigt hatten [453-455], konnte in mehreren großen und methodologisch einwandfreien Studien sowie in einer Metaanalyse kein klinisch signifikanter Benefit bezüglich Asthma-bezogener Endpunkte demonstriert werden [456-460].

\subsection{Asthma und obstruktives Schlafapnoesyndrom}

Asthma und obstruktives Schlafapnoesyndrom (OSAS) sind in epidemiologischen Studien miteinander assoziiert [448, 461 463]. Die obstruktive Schlafapnoe ist eine mögliche Ursache für eine schlechte Asthmakontrolle [464]. Gemäß den entsprechenden schlafmedizinischen Leitlinien [465] sollte die symptomatische Schlafapnoe diagnostiziert und behandelt werden. Die Behandlung einer asymptomatischen Schlafapnoe zur Verbesserung Asthma-bezogener Zielparameter ist durch Daten nicht ausreichend belegt.

\subsection{Asthma und endokrinologische Störungen}

Ein niedriger Vitamin-D-Spiegel und Asthma, insbesondere schweres Asthma, sind in epidemiologischen Studien miteinander assoziiert. Bei Patienten mit Asthma findet sich oft ein Vitamin-D-Mangel [466]. Ein niedriger Vitamin-D-Spiegel ist bei Patienten mit Asthma mit einer schlechteren Lungenfunktion assoziiert [467]. Die mütterliche Vitamin-D-Aufnahme ist invers mit der Asthmainzidenz bei Kindern assoziiert [468]. Prospektive Studien, ob eine Vitamin-D-Gabe die Inzidenz oder die Kontrolle eines Asthmas beeinflusst, sind bislang nicht verfügbar.

\subsection{Asthma und Adipositas}

Adipositas und Asthma sind in epidemiologischen Studien miteinander assoziiert, eine Adipositas geht mit vermehrter Atemwegsentzündung [469], gesteigerter bronchialer Hyperreagibilität [470-472] und verschlechterter Asthmakontrolle einher [447, 448, 451, 473-500], wobei Frauen hier empfindlicher zu sein scheinen [501]. Bei adipösen Kindern ist das Ansprechen auf eine Glucocorticosteroid-Therapie vermindert [502]. In einer Studie konnte keine Häufung von leichtem Asthma bei Patienten mit Adipositas gesehen werden [503]. Bei Kindern spielt vor allem eine stammbetonte Adipositas eine Rolle [504]. Insulinresistenz ist ebenfalls mit Asthmasymptomatik vergesellschaftet [505]. Die Behandlung einer ausgeprägten Adipositas durch bariatrische Therapie hat einen positiven Effekt auf die Kontrolle eines Asthma bronchiale [441, 506 - 508].

\subsection{Asthma und COPD}

Bei den zahlreichen Phänotypen von obstruktiven Atemwegsund Lungenerkrankungen gibt es zunehmend Hinweise dafür, dass die Schnittmenge von COPD und Asthma klinisch relevant ist. Klinisch kann der „Overlap-Phänotyp“ durch eine nur inkomplett reversible Atemwegsobstruktion (Zeichen der COPD) in Kombination mit einem deutlichen Ansprechen auf Bronchospasmolyse bzw. auf Testung der bronchialen Hyperreagibilität [509] oder als neu diagnostizierte COPD bei Patienten mit einem vorbestehenden Asthma [510] definiert werden. International wurde in einer Stellungnahme der Global Initiative for Obstructive Lung Disease (GOLD) zusammen mit der Global Initiative for Asthma (GINA) eine Reihe von Kriterien aufgeführt (anamnestisch, lungenfunktionsanalytisch, bildmorphologisch, laborchemisch), welche für Asthma bzw. für COPD typisch sind [183]. Wenn auf einen Patienten ähnlich viele Kriterien für Asthma bzw. für COPD zutreffen, so sollte die Bezeichnung „Asthma-COPD-Overlap“ („ACO“) in Erwägung gezogen werden. Der Ansatz wird kritisch diskutiert, da der Begriff unscharf definiert ist, sodass sich die Diagnose ACO auf eine Vielzahl von Patienten mit unterschiedlichem Phänotyp anwenden lässt. Es handelt sich beim ACO um keine eigenständige Krankheitsentität. 
Schätzungen in vielen Kohorten weisen darauf hin, dass eine Überlappung von COPD und Asthma bei ca. 10\%-50\% der COPD-Patienten [121,510-527] und bei ca. 10\%-60\% der Asthmapatienten $[121,509,511,513,515,517,520,522,525$, 528] anzutreffen ist. Die beträchtliche Bandbreite der berichteten Prävalenz ist Folge der heterogenen Definitionen, welche für die jeweiligen Untersuchungen verwendet wurden.

Die klinische Relevanz des gemeinsamen Auftretens eines Asthmas und einer COPD ergibt sich aus einer Vielzahl an Daten, die eine erhöhte Symptomlast, vermehrte Exazerbationen und vermehrte Hospitalisierungen bei solchen Individuen feststellen, die unter beiden Erkrankungen leiden [510, 520, 522]. Darüber hinaus gibt es Anzeichen dafür, dass ACO-Patienten auch eine gegenüber den beiden Einzelerkrankungen erhöhte Mortalität aufweisen [528].

Bezüglich der Therapie gibt es Hinweise dafür, dass COPD mit einer begleitenden asthmatischen Komponente durch ein besseres Ansprechen auf inhalative Glucocorticosteroide gekennzeichnet ist [529-531]. Dabei ist der Stellenwert verschiedener Biomarker, insbesondere der von Bluteosinophilen, Gegenstand anhaltender und intensiver Forschungsbemühungen [532-538].

\subsection{Asthma und Rhinitis}

Asthma bronchiale und chronisch-entzündliche Erkrankungen der oberen Atemwege (EOA: u.a. Rhinitis, Sinusitis, Polyposis nasi) sind häufig miteinander vergesellschaftet [539-546]. Die Evidenz aus epidemiologischen Untersuchungen deutet auf eine enge Verbindung dieser Erkrankungen hin. Mehr als $80 \%$ der Patienten mit Asthma bronchiale haben eine EOA und bis zu $40 \%$ der EOA-Patienten ein koexistierendes Asthma [539, 541]. Daher sollte bei Patienten mit Asthma immer auch eine Beteiligung der oberen Atemwege diagnostiziert werden sowie umgekehrt [539-542]. Einige Autoren gehen so weit, bestimmte Untergruppen von Asthma bronchiale und Rhinitis, Sinusitis und Polyposis nasi nicht mehr als getrennte Erkrankungen zu definieren, sondern als Schleimhauterkrankungen mit gleichartiger Pathophysiologie im Bereich der oberen und unteren Atemwege, also als „United Airways“-Erkrankungen [539].

Entzündliche Erkrankungen im Bereich der oberen und unteren Atemwege können IgE-vermittelt auftreten und durch identische Trigger (Allergene wie beispielsweise Pollen, Milben, Schimmelpilze, Tierepithelien und Bakterien) ausgelöst werden [541, 542].

Erkrankungen der oberen Atemwege sind ein Risikofaktor für die Entstehung eines Asthmas bzw. können den Verlauf eines Asthmas negativ beeinflussen und sollten daher adäquat diagnostiziert und therapiert werden. Hierbei können die Entwicklungen beider Erkrankungen parallel verlaufen oder eine Manifestation geht der anderen voraus. In einer 10-Jährigen Longitudinalstudie bei Kindern ging in 19\% der Fälle die Entwicklung einer EOA der Asthmaentstehung voraus, in $25 \%$ entwickelten sich EOA und Asthma gleichzeitig [541]. In einer anderen Studie, in der mehr als 2000 Studenten über 23 Jahre nachuntersucht wurden, entwickelten die EOA-Patienten 3mal häufiger ein Asthma als die Nicht-EOA-Kontrollpopulation
[543]. Eine finnische Zwillingsstudie über 15 Jahre zeigte, dass männliche EOA-Patienten ein 4-fach höheres Risiko für eine Asthmaentstehung hatten, die weiblichen sogar ein 6-fach höheres Risiko im Vergleich zu Kontrollen ohne EOA [544]. Guerra et al. [540] fanden nach Adjustierung auf Geschlecht, Alter, atopischen Status, Jahr der Nachbeobachtung, Rauchverhalten und evtl. Vorhandensein einer COPD eine EOA ebenfalls als hochgradigen unabhängigen Risikofaktor für Asthma. Auch unabhängig von der Atopie als gemeinsame Grundlage waren EOA und Asthma statistisch hochsignifikant verbunden [540].

Ebenso wurde nachgewiesen, dass bei der Mehrzahl der Patienten mit manifester oberer Atemwegserkrankung, aber ohne klinisches Asthma, nach einer bronchialen Methacholin-Provokation bereits eine bronchiale Hyperreaktivität nachweisbar war [546 - 550] und dass der Anstieg der bronchialen Reaktivität bei Patienten mit einer allergischen Rhinitis auf Pollen mit der Pollenexposition in der Saison verbunden war [545, 546]. Auch konnte bei 2/3 der Patienten mit einer allergischen Rhinitis eine Erweiterung der peripheren Bronchien nach Bronchospasmolyse-Testung nachgewiesen werden, obwohl diese Patienten hinsichtlich der unteren Atemwege bislang klinisch nicht aufällig waren und eine normwertige $\mathrm{FEV}_{1}$ vor Testung aufwiesen [546, 547].

Die Kombination aus Nasenpolypen und Asthma tritt typischerweise als "late-onset-disease“ auf, wobei die gleichen Typ 2-vermittelten eosinophilen Entzündungsvorgänge in den oberen und unteren Atemwegen (und z. T. auch in den Mittelohren) auftreten.

Neben einer bronchialen Hyperreaktivität wurde nach nasaler Allergenprovokation bei Patienten mit allergischer Rhinitis (AR) auch ein Anstieg von Eosinophilen im Sputum nachgewiesen [550]. Bei Entnahme von nasalen und bronchialen Probebiopsien 24 Stunden nach einer nasalen Allergenprovokation konnte sowohl in den nasalen wie auch in den bronchialen Epithelien eine Einwanderung von Eosinophilen gezeigt werden [551]. Umgekehrt führten auch bronchiale Allergenprovokationen bei nicht asthmatischen Patienten mit allergischer Rhinitis zu einer inflammatorischen Veränderung der Nasenschleimhaut und einem Anstieg eosinophiler Zellen im peripheren Blut [552]. Auch bei Asthmapatienten ohne EOA war eine erhöhte Eosinophilenzahl in nasalen Biopsien nachweisbar [553]. Die Eosinophileninfiltration in obere und untere Atemwege wird gleichartig durch inflammatorische Zytokine induziert, speziell durch Interleukin-5 [554, 555]. Andere entzündliche Mechanismen als reine IgE-vermittelte Erkrankungen wurden auch in 12 fortlaufenden europäischen prospektiven Geburts-Kohortenstudien an 4-8-jährigen Kindern im Rahmen des MeDALL-Studienprojekts (Mechanisms of the Development of ALLergy) vermutet [556]. Die Komorbidität von Rhinitis, Asthma und Ekzemen bei diesen Kindern wurde erfasst und mittels longitudinaler Analysen in einem Log-linear-Modell mit einer errechneten Komorbidität verglichen. Bei 16147 Kindern im Alter von 4 Jahren war das relative Risiko für Asthma, Rhinitis und Ekzeme bei 4-jährigen Kindern 63,5\%. Eine IgE-vermittelte Erkrankung konnte jedoch nur bei $38 \%$ der Kinder nachgewiesen werden, weshalb andere Mechanismen für die hohe Komorbidität der Erkrankung angenommen werden müssen [556]. 
Leitlinien empfehlen topische nasale Glucocorticoide als Basis der Therapie einer allergischen Rhinitis [557]. Die Therapie einer allergischen Rhinitis oder eines Asthmas kann potenziell die jeweils andere koexistente Erkrankung positiv beeinflussen hinsichtlich Krankheitskosten, Exazerbationen, Notfallbesuchen oder Arbeitsausfällen. Dies wurde erstmals im Jahr 1987 beschrieben, als für die intranasale Anwendung von Beclomethason und Flunisolid bei Patienten mit AR und Asthma erstmals eine deutliche Reduktion der selbstbeobachteten Asthmasymptome Atemnot und Giemen beschrieben wurde [558].

Eine 4-wöchige Applikation von nasalem Budesonid reduzierte den Schweregrad eines anstrengungsinduzierten Asthmas bei Kindern [559] und eine 5-wöchige Gabe von Beclomethason-Nasenspray reduzierte die bronchiale Hyperreaktivität bei Erwachsenen [560]. In einer anderen Crossover-Studie hatten Patienten mit AR, aber ohne Asthma, nach 2 Wochen einer intranasalen Anwendung von Beclomethason eine reduzierte bronchiale Hyperreaktivität, aber interessanterweise keine Änderung der bronchialen Hyperreaktivität nach nur bronchialer Anwendung von Beclomethason [561]. Auch wenn diese Studien Hinweise darauf geben, dass die Behandlung einer koexistierenden AR mit nasalen Kortikosteroiden bei der Asthmakontrolle helfen kann, ist darauf hinzuweisen, dass die obengenannten Studien relative kleine Patientenzahlen eingeschlossen hatten (11 bis 26 Patienten).

In einer größeren Studie [562] an 262 Patienten konnten im Vergleich von intranasalem Fluticason, inhaliertem Fluticason, deren Kombination oder inhaliertem Placebo nur die Gruppen mit inhaliertem Fluticason hinsichtlich ihrer bronchialen Hyperreaktivität profitieren. In einer weiteren Studie an Asthmapatienten $(n=160)$ [563] profitierte ein Teil der Patienten mit schwerem Asthma und chronischer Rhinosinusitis (CRS). Mittels computertomografischer Darstellungen der Nasennebenhöhlen wurde bei den Asthmatikern nach dem Lund-MackayScoring eine CRS bei 66,9\% ( $n=107)$ der Patienten diagnostiziert und diese CRS-Patienten hatten signifikant häufiger ein schweres Asthma als die Patienten ohne CRS. Eine intranasale Glucocorticosteroid-Therapie mit Mometasonfuroat wurde allen 107 Patienten erstmalig bei Studieneinschluss verabreicht und mittels Asthma Control Test (ACT), Asthma Control Questionnaire mit 5 items (ACQ5), Spirometrie und Kontroll-CT der Nasennebenhöhlen nach 3-monatiger Behandlung kontrolliert. Bei 24 von 107 (22,4\%) Patienten verbesserten sich alle Asthmaparameter (ACT, ACQ5 und FEV $_{1}$ ) signifikant nach der 3-monatigen additiven intranasalen Glucocorticosteroid-Behandlung bei weitgehend unverändertem Lund-Mackay-Scoring in der NNH-CT.

Während $\mathrm{H}_{1}$-Antihistaminika zu den Standardtherapeutika in der Therapie der AR gehören, spielen sie in der Behandlung des Asthmas eine untergeordnete Rolle. Es existieren nur einige wenige Studien, die eine allenfalls sehr geringe Wirkung von Antihistaminika auch beim Asthma postulieren [564 - 566].

Die AR-Behandlung bei Asthmatikern kann Krankheitskosten einsparen. Die Rate an Krankenhauseinweisungen und Notfallbehandlungen aufgrund ihres Asthma bronchiale konnte bei Asthmatikern mit einer suffizienten AR-Behandlung $(n=3587)$ signifikant reduziert werden im Vergleich zu einem identischen Kollektiv von Patienten mit Asthma und AR ohne AR-Therapie ( $n=1357$ ) [567]. Asthma-bedingte stationäre Aufnahmen gingen von 2,3 auf 0,9 zurück (61\% Reduktion) und die Inzidenz von Notfallbehandlungen aufgrund des Asthmas von 1,3 auf 0,6 je Patient (54\% Reduktion) [567]. Diese Ergebnisse konnten in einer zweiten Studie mit nasalen GCS als AR-Therapie bestätigt werden [568].

\subsection{Asthma und Psyche}

Psychische Komorbiditäten, insbesondere Angsterkrankungen und Depressionen, sind bei Asthmapatienten häufig [569571]. In vielen Fällen werden psychische Komorbiditäten/psychosoziale Belastungen allerdings nicht erkannt bzw. nicht adäquat behandelt [572].

Psychische Komorbiditäten können neben der Pathophysiologie des Asthmas (siehe Kapitel Endogene Faktoren) auch Symptomwahrnehmung, Compliance und Leistungsinanspruchnahme beeinflussen und somit die Behandlung und das Selbstmanagement erschweren sowie das Erreichen einer guten Kontrolle verhindern [2,573-578]. Zudem können psychische Faktoren auch die zentralnervöse Verarbeitung von asthmatischen Symptomen beeinflussen [579, 580].

Bei gleichzeitigem Vorliegen eines schweren Asthmas und psychischen Komorbiditäten oder ungünstigen psychosozialen Faktoren kann ein erhöhtes Mortalitätsrisiko gegeben sein [393].

Bei Kindern/Jugendlichen können krankheitsbedingte Auswirkungen die körperlich/seelische und schulische Entwicklung sowie den psychosozialen Rahmen betreffen [581]. Das familiäre und auch soziale Umfeld von Kindern/Jugendlichen kann über physiologische wie auch verhaltensbezogene Mechanismen das Krankheitsgeschehen deutlich beeinflussen [38, 575, 582] und sich entscheidend auf die Akzeptanz der Diagnose und der notwendigen therapeutischen Maßnahmen auswirken. In Anamnese und Behandlungskonzept sollten daher psychische Komorbiditäten und psychosoziale Problemkonstellationen berücksichtigt werden und ggf. psychologische bzw. psychotherapeutische Hilfen angeboten werden. Dies gilt insbesondere für Patienten mit unzureichender Asthmakontrolle [393,583]. Diagnostik, Beratung, Schulung und Therapie bei ungünstigen psychischen und psychosozialen Konstellationen sind etablierte Elemente in der pneumologischen Rehabilitation bei Kindern, Jugendlichen und Erwachsenen mit Asthma (siehe Kapitel zur Rehabilitation) [401, 584-586].

\section{Prävention}

Bei der Prävention wird unterschieden in primäre, sekundäre und tertiäre Prävention [588-591].

Das Ziel der primären Prävention ist es, die Entwicklung der Erkrankung zu verhindern, bevor die immunologischen Weichen für die Erkrankung gestellt sind. Nur diese wird im Folgenden behandelt. Die sekundäre Asthmaprävention zielt auf die Vermeidung der Entwicklung von Asthma bei Kindern, die bereits eine Sensibilisierung bzw. eine andere Art der allergischen Manifestation, z. B. Nahrungsmittelallergie oder atopische Der- 
EMPFEHLUNGEN

E68 Neugeborene sollten vier Monate ausschließlich gestillt werden.

E69 Nach dem vollendeten 4. Lebensmonat soll Beikost eingeführt werden; es soll keine Verzögerung der Beikosteinführung erfolgen.

E70 Kinder mit Allergierisiko sollten keine Fell- und Federtiere anschaffen bzw. halten. Katzenhaltung ist bei diesen Kindern generell nicht zu empfehlen.

E71 Hohe Luftfeuchtigkeit und mangelnde Ventilation in Räumen, flüchtige organische Verbindungen (z.B. Formaldehyd), aktive und passive Tabakrauchexposition sowie Kfz-Abgasexposition sollen vermieden werden.

E72 Auch Säuglinge und Kinder mit erhöhtem Asthmarisiko sollen nach den allgemeinen Empfehlungen geimpft werden.

E73 Übergewicht und Adipositas sind mit Asthma positiv assoziiert und sollten bei Kindern vermieden werden.

S17 Es gibt keinen Anhalt dafür, dass der Einsatz partiell oder extensiv hydrolisierter Säuglingsnahrungen der Asthmaprävention dient.

S18 Es sind keine diätetischen Restriktionen im Hinblick auf die Ernährung der Mutter während der Schwangerschaft und Stillzeit erforderlich.

S19 Kinder ohne Allergierisiko müssen nicht auf Haustiere verzichten.

S20 Eine Hausstaubmilbensanierung ist zur Primärprävention nicht zu empfehlen, zur Sekundär- und Tertiärprävention jedoch ratsam.

S21 Eine höhere Anzahl älterer Geschwister, der Besuch einer Kindertagesstätte, das Aufwachsen auf einem Bauernhof mit Tieren sowie Wurminfektionen sind negativ mit Asthma assoziiert.

S22 Aktive Tabakrauchvermeidung und striktes Meiden von Environmental Tobacco Smoke (ETS) ist die wichtigste Präventions-Maßnahme in der Schwangerschaft [587].

matitis haben. Hierzu zählt die Expositionsminimierung, aber auch die spezifische Immuntherapie bei allergischer Rhinitis, um die Entwicklung zum Asthma zu verhindern. Tertiäre Prävention zielt auf die Verhinderung der Verschlimmerung eines bereits bestehenden Asthmas und beinhaltet z. B. eine Umgebungssanierung sowie eine Pharmakotherapie.

Präventionsansätze der primären Prävention Trotz der besseren Kenntnis von Risikofaktoren, Schutzfaktoren oder Determinanten ist unser Verständnis vom natürlichen Verlauf des Asthmas noch recht unvollkommen. Derzeit erscheinen viele Erkenntnisse, die mithilfe der sogenannten Hygienehypothese gewonnen wurden, biologisch plausibel [592-596]. Trotzdem sind die Ergebnisse aus epidemiologischen und besonders aus Interventionsstudien häufig kontrovers [597]. Der wahrscheinlichste Grund dafür ist die sehr komplexe Gen-Umwelt-Inter- aktion, für die neben den bekannten Umweltfaktoren unterschiedliche Major- und Minorgene entscheidend sind.

Da primäre Prävention durch genetische Manipulation derzeit noch nicht möglich ist und auf absehbare Zeit auch nicht möglich sein wird, ist daher für einen sinnvollen Präventionsansatz die Identifikation von veränderbaren Faktoren in der Umwelt des Kindes notwendig. Nur in wenigen Bereichen haben sich aber Präventionsmaßnahmen bislang als möglich und sinnvoll erwiesen.

Das vulnerable Fenster (window of opportunity) für die Entwicklung eines Asthmas liegt in utero und im sehr frühen Kindesalter. Daher sollten primäre präventive Interventionsmaßnahmen ebenfalls früh, möglicherweise schon während der Schwangerschaft begonnen werden, um eine Chance auf Erfolg zu haben. Im Wesentlichen erstrecken sich diese Maßnahmen auf die Reduktion von Passivrauchexposition und auf die Stärkung natürlicher Schutzfaktoren, die als Folge der modernen Zivilisation zugunsten von Risikofaktoren abgenommen haben. Studien, die diese Empfehlungen belegen, sind allerdings rar.

\subsection{Ernährung}

Zur primären Prävention durch Ernährung sind die folgenden Ansätze in klinischen Studien geprüft:

- Muttermilch

- allergenreduzierte Diät der Mutter in der Schwangerschaft und Stillperiode

- hypoallergene Säuglingsnahrung

- Immunonutrients

- späte Einführung von Beikost

Muttermilch Allgemein anerkannt ist, dass Muttermilch aus vielfältigen Gründen die beste Ernährung für Säuglinge ist. Es wird deshalb empfohlen, Säuglinge für 4- 5 Monate ausschließlich mit Muttermilch zu ernähren. Für das Asthma bronchiale ist ein präventiver Einfluss nicht schlüssig belegt [598, 599].

Allergenreduzierte Diät der Mutter in der Schwangerschaft und Stillperiode In mehreren Interventionsstudien wurde versucht, den Allergengehalt in der Ernährung der Schwangeren sowie der Mutter während der Stillzeit und zusätzlich die Belastung mit Inhalationsallergenen (in erster Linie durch spezielle Hausstaubmilbensanierungen) zu reduzieren, um Allergieprävention zu betreiben [600, 601]. Zusätzlich wurde in einigen dieser Studien auch die Passivrauchbelastung deutlich vermindert. Dadurch konnte eine gewisse Reduktion der Asthmahäufigkeit im späteren Leben erzielt werden. Wurde nur der Allergengehalt in der Ernährung der Mutter während der Stillzeit oder Schwangerschaft reduziert, konnte kein asthmapräventiver Effekt erreicht werden.

\section{Hypoallergene Säuglingsnahrung zur Atopieprävention} (Hydrolysatnahrungen) Unter Hydrolysatnahrungen (HANahrungen) werden Säuglingsnahrungen verstanden, deren Antigengehalt durch Bearbeitung der Nahrungsproteine mithilfe lebensmitteltechnologischer Verfahren (enzymatische Spaltung, Ultraerhitzung, Ultrafiltration) in unterschiedlichem Ausmaß reduziert wurde. Die Vielfalt der verfügbaren Hydrolysat- 
nahrungen ist groß und verwirrend. Grundsätzlich lassen sie sich jedoch relativ einfach in starke und schwache bzw. partiell und extensiv hydrolysierte Nahrungen sowie in Molken- und Kaseinhydrolysate einteilen. Erstere Unterscheidung beruht auf der Art der Bearbeitung und dem resultierenden Restantigengehalt, gekennzeichnet durch das Molekulargewicht, letztere ist bedingt durch das Protein, aus welchem die Formula aufgebaut ist.

Zahlreiche Studien in den letzten Jahren hatten gezeigt, dass sowohl extensiv als auch partiell hydrolysierte Säuglingsnahrungen einen allergiepräventiven Effekt haben können, der sich auf die Verringerung der Inzidenz des atopischen Ekzems, aber nicht des Asthmas bezieht. Eine aktuelle Metaanalyse fand allerdings keinen signifikanten präventiven Effekt von Hydrolysatnahrungen auf die Asthmaprävalenz [602]. In der GINI-Studie konnte eine marginale Reduktion der Asthmaprävalenz für die Jahre 10-15 unter einer extensiven Caseinhydrolysatnahrung (eHA-C) gefunden werden. Die kumulative Asthmaprävalenz wurde durch keine der verwendeten HA-Nahrungen reduziert [603-607].

Immunonutrients Ein neuer Ansatz zur nutritiven Allergieprävention ist die Supplementierung der mütterlichen Diät in Schwangerschaft und Stillzeit bzw. der kindlichen Nahrung mit sogenannten Immunonutrients. Zu diesen in prospektiven, kontrollierten klinischen Studien geprüften Substanzen zählen Pro- und Präbiotika und ungesättigte Fettsäuren (Omega-3 Fettsäuren ( $\omega$-3-PUFAs poly-unsaturated fatty acids)). Eine aktuelle Studie zeigte, dass eine tägliche Einnahme von langkettigen PUFAs durch Mütter im letzten Schwangerschaft-Trimenon das Asthmarisiko der geborenen Kinder senkt [79]. Mütterliche Einnahme von Vitamin D und E während der Schwangerschaft ist assoziiert mit einem geringeren Risiko für Erkrankungen mit obstruktiver Ventilationsstörung [76, 608-616].

Späte Beikost In einigen (wenigen) älteren Studien konnte ein präventiver Effekt auf die Asthmaprävalenz im Kindesalter durch die späte Einführung wenig diversifizierter Beikost nachgewiesen werden. Analysen von prospektiv geführten Ernährungsprotokollen aus neuen Geburts-Kohorten-Studien zeigen allerdings, dass „reverse causality“ (=Umkehr von Ursache und Folge) bei der Beurteilung der Beikosteinführung hinsichtlich des allergiepräventiven Effektes berücksichtigt werden soll. Sowohl in der GINI-Studie als auch der LISA-Studie konnte kein präventiver Effekt bezüglich einer verzögerten Beikosteinführung, insbesondere auch von besonders potenten Nahrungsmittelallergenen, gezeigt werden $[617,618]$. Eine verzögerte Beikosteinführung über den vollendeten 6. Lebensmonat hinaus und die Meidung potenter Nahrungsmittelallergene im 1. Lebensjahr sowie eine spezielle Diät nach dem 1. Lebensjahr wird in neueren Leitlinien daher nicht mehr empfohlen werden [557].

\subsection{Kontrolle des Körpergewichts}

Insgesamt sprechen die verfügbaren Studien dafür, dass Adipositas die Entwicklung eines Asthmas fördert und bei bestehendem Asthma den Schweregrad steigert. Deshalb soll Überge- wicht vermieden und adipösen Asthmatikern eine Gewichtreduktion dringend empfohlen werden [619-623].

\subsection{Innenraumbelastung}

Sensibilisierung gegenüber Innenraum-Aeroallergenen hat mehr Gewicht für die Entwicklung von Asthma als AußenraumAeroallergene.

\subsection{Tabakrauchexposition}

Die Vermeidung der aktiven und passiven Tabakrauchexposition zählt zu den wichtigsten Maßnahmen der Primärprävention bei Asthma. Aktives Rauchen führt zu einem gesteigerten Asthmarisiko. Zahlreiche Studien belegen darüber hinaus, dass das Rauchen der Mutter während der Schwangerschaft, mütterliches Rauchen danach, väterliches Rauchen und das Rauchen beider Eltern das Asthmarisiko beim Kind erhöhen. Aktive und passive Tabakrauchexposition sollen deshalb strikt vermieden werden [624-631].

\subsection{Inhalationsallergene}

Der Zusammenhang zwischen der Exposition gegenüber Inhalationsallergenen und einer späteren Sensibilisierung bzw. dem Auftreten allergischer oder asthmatischer Symptome ist sehr komplex. In einigen Studien konnte gezeigt werden, dass dabei nicht immer eine lineare, sondern eine glockenförmige Dosis-Wirkungsbeziehung bestehen kann. Das heißt, dass eine sehr geringe Allergenexposition und eine sehr hohe Allergenexposition präventiv für eine Sensibilisierung sein können, während eine Exposition im mittleren Bereich ein deutliches Risiko darstellt. Auch ist die Höhe der Exposition gegenüber Katzenallergen oder Hausstaubmilbenallergen in den ersten 6 Monaten nicht mit dem Auftreten eines klinischen Asthmas im Alter von 6 bis 7 Jahren assoziiert. Andererseits konnte in einer anderen Studie gezeigt werden, dass eine frühere hohe Exposition gegenüber Hausstaubmilben mit Lungenfunktionseinschränkungen im Alter von 10 Jahren assoziiert ist [49,632].

\subsection{Hausstaubmilben}

Zahlreiche Interventionsstudien, die eine Hausstaubmilbensanierung (ohne zusätzlich andere Maßnahmen) als Form einer primären Prävention für die Asthma- oder Allergieentstehung untersuchten, konnten keinen solchen Effekt nachweisen. Daher kann keine Empfehlung zur primären Asthmaprävention mittels Hausstaubmilbensanierungen (z. B. Encasing) gegeben werden, zumal auch die Sensibilisierung keine lineare Beziehung zum Grad der Hausstaubmilbenexposition aufweist [633 $-641]$.

\subsection{Haustiere}

Die Frage, ob Haustiere allergiefördernd oder -präventiv wirken, ist zum jetzigen Zeitpunkt nicht eindeutig geklärt. Es gibt widersprüchliche Studienergebnisse bezüglich der Auswirkungen einer Haustierexposition im frühen Kindesalter auf die Asthmaentstehung [642-653].

Insgesamt überwiegen bei der Haltung von Katzen, Kleintieren und Nagern die Studien, die in der Haltung einen Risikofaktor sehen. Deshalb sollte in Risikopopulationen (Atopie beider 
Eltern) die Haltung dieser Tiere vermieden werden. Mehrere Studien zeigen dagegen, dass frühe Hundehaltung bei Kindern die Asthmahäufigkeit reduzierte $[654,655]$.

Es scheint aber, dass ein möglicher präventiver Effekt von früher Hundehaltung nur bei Kindern zu sehen ist, die nicht aus einer asthmabelasteten Familie stammen. Es sind bislang sicher nicht ausreichend Studien vorhanden, die die frühe Hundehaltung als Präventivmaßnahme rechtfertigen würden. Zudem ist nicht klar, ob das Hundeallergen oder vielmehr das durch den Hund erhöhte Endotoxin oder ähnlich wirkende Immunomodulatoren im Haus den präventiven Effekt in den Studien verursacht haben [656].

\subsection{Schimmel und Feuchtigkeit}

Ein Innenraumklima, das Schimmelpilzwachstum fördert, wie hohe Luftfeuchtigkeit und mangelnde Ventilation, erhöht das Asthmarisiko [657-661]. Sinnvolle Maßnahmen zur Sanierung und Vermeidung von Schimmelpilzwachstum bzw. feuchten Stellen in Wohnräumen sind deshalb zu empfehlen. Beim Bundesumweltamt ist ein entsprechender Leitfaden erhältlich.

\subsection{Außenraumbelastung}

Kfz-Emission und Wohnen Das Wohnen an einer stark befahrenen Straße ist durch die Exposition gegenüber zahlreichen Schadstoffen wie z.B. Stickoxiden und kleinen Partikeln mit einem erhöhten Asthmarisiko verbunden. In Abhängigkeit von dem Abstand zur Straße und ihrer Verkehrsdichte steigt das Risiko. Es wird deshalb empfohlen, die Exposition gegenüber Kfz-Emissionen gering zu halten [662-666].

Insgesamt scheint die Reduktion nur eines Allergens oder Schadstoffs nutritiver oder inhalativer Provinienz nur eine geringere bis keine Rolle für die Asthmaentwicklung zu spielen, dagegen hat eine Intervention, die mehrere asthmarelevante Komponenten vermindert, sehr wohl eine Asthmareduktion zur Folge [603, 667-672].

\subsection{Weitere Empfehlungen}

Medikamente während der Schwangerschaft, bei Säuglingen und jungen Kleinkindern Mehrere Studien - wenn auch nicht alle - haben gezeigt, dass Breitbandantibiotika und Paracetamol verabreicht in der Schwangerschaft und ersten Lebensphase das Asthmarisiko im späteren Leben erhöhen. Es wird empfohlen, diese Medikamente nur bei eindeutiger Indikation einzusetzen [575,673-677]. Eine vaginale Geburt geht gegenüber der sectionalen Geburt wahrscheinlich mit einer geringeren Asthmahäufigkeit einher [678, 679].

Impfungen Impfungen erhöhen nicht das Allergie- und Asthmarisiko, eventuell senken sie es aber. Es wird deshalb empfohlen, dass alle Kinder nach den allgemeinen Empfehlungen geimpft werden.

Asthmaassoziationen Bedingungen, die zwar negativ mit Asthma assoziiert sind, für die sich in Bezug auf die Primärprävention von Asthma aber (noch) keine unmittelbaren Präventionsempfehlungen ableiten lassen, sind: eine höhere Anzahl älterer Geschwister, der Besuch einer Kindertagesstätte, das
Aufwachsen auf einem Bauernhof mit Tieren sowie Wurminfektionen.

Präventionsforschung Asthmaimpfung. Einen interessanten und logischen Ansatz zur Asthmaprävention stellen Versuche dar, eine Asthmaimpfung zu entwickeln, die aus einem parenteral verabreichten Cocktail aus Inhalationsallergenen in Kombination mit Adjuvantien besteht, die speziell die TH1-Antwort stimulieren. Derartige Ansätze aus Tierversuchen sind interessant, weil sie darauf abzielen, die Th1-Th2-Balance in Richtung Th1-Immunantwort zu triggern. Bislang existiert diese Impfung für den Menschen noch nicht, darüber hinaus ist nicht bekannt, ob sie auch bei bereits eingetretener Sensibilisierung im Sinne einer Immunmodulation von einer Th2-zu einer Th1-gewichteten Immunantwort wirken würde.

BCG-Impfung. Es gibt Hinweise aus Mausmodellen, dass es möglich ist, mit der BGC-Impfung eine immunologische Deviation in die Richtung eines Th1-Phänotyps zu bewirken und damit die allergische Sensibilisierung, die Atemwegsinflammation und -hyperreagibilität zu supprimieren. Klinische Studien am Menschen liegen dazu noch nicht vor $[680,681]$.

FAZIT Zu meiden sind Tabakrauch- und Kfz-Abgasexposition, feuchtes Innenraumklima, Tierhaar- und Hausstaubmilbenexposition bei nachgewiesener Sensibiliserung und klinischen Symptomen, Übergewicht sowie Breitbandantibiotika und Paracetamol während der Schwangerschaft und der ersten Lebensphase - wenn vertretbar! Eine vaginale Entbindung sollte, soweit möglich, angestrebt werden.

\section{Arbeitsbedingtes Asthma}

EMPFEHLUNGEN

E74 Asthmasymptome des Erwachsenen sollten Anlass für eine eingehende Arbeitsanamnese sein, da in 9-25\% der Fälle berufliche Auslöser vorliegen und deren Identifikation für die Therapie und Prävention bedeutsam ist. E75 Arbeitsplatzgrenzwerte von bekannten Asthmaauslösern sollen eingehalten werden.

E76 Bei erhöhtem Erkrankungsrisiko (vorbestehende Atemwegsallergien, unspezifische bronchiale Hyperreagibilität) und Exposition gegenüber Auslösern des Berufsasthmas sollten regelmäßige arbeitsmedizinische Vorsorgeuntersuchungen (Surveillance) erfolgen; dies gilt insbesondere bei Vorliegen von unzureichenden arbeitshygienischen Maßnahmen.

S23 Es gibt über 550 verschiedene Auslöser des Berufsasthmas, wobei es sich in 2/3 der Fälle um Allergene, in $1 / 3$ um Irritantien handelt.

S24 Im Falle eines berufsbedingten Asthmas ist die strikte Karenz gegenüber der ursächlichen Noxe (das heißt ggf. Arbeitsplatzwechsel oder sogar Umschulung) die einzige erfolgversprechende (interventionelle) Therapiemaßnahme. 
- Tab.13 Arbeitsbereiche mit besonderer Gefährdung für die Entstehung eines arbeitsbedingten Asthmas.

\section{Gefährdung durch vorrangig allergisie- rend wirkende Arbeitsstoffe}

Bäckerei, Konditorei, Mühle, Gärtnerei, Plantagen-, Dock- und Lagerarbeit, Küchenbetriebe (enzymhaltige Fleischweichmacher, Nahrungsmittelaerosole), Obstverwertung, pharmazeutische Industrie, industrielle und Forschungs-Laboratorien, Veterinärwesen, Geflügelfarmen, Futter- und Nahrungsmittelindustrie, Imkerei
Gefährdungen durch vorrangig chemischirritativ oder toxisch wirkende Arbeitsstoffe

Polyurethanweichschaum- und -hartschaumherstellung, Lackiererei, Herstellung und Schweißen von PVC-Folien, -Platten und -Röhren, Lötarbeiten, Desinfektionsmittel-Einsatz, Galvanisierbetriebe, Metallveredelung, Zementherstellung und -verarbeitung, Schweißen, Färberei, Textil- und Friseurbetriebe
Gefährdung durch Arbeitsstoffe mit vorrangig unbekanntem Pathomechanismus

Sägerei, Kunststoffherstellung, Stallungen, Elektronikindustrie, chemische und pharmazeutische Industrie

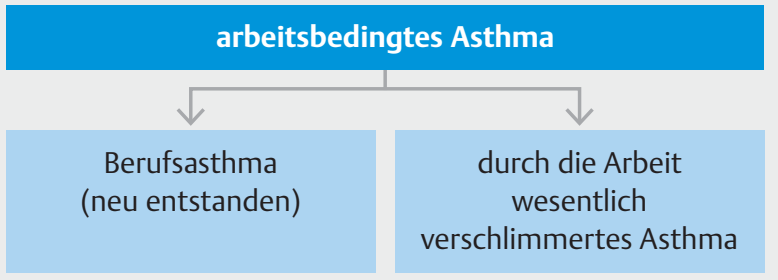

Pathomechanismus

- IgE-vermittelt (allergisch)

- irritativ

- unbekannt

- Abb. 3 Unterteilung des arbeitsbedingten Asthmas und zugrunde liegende Pathomechanismen.
- Tab.14 Untergruppen des arbeitsbedingten irritativen Asthmas. Siehe Text für Einzelheiten. AGW = Arbeitsplatzgrenzwert.

\begin{tabular}{|l|l|l|}
\hline Untergruppe & $\begin{array}{l}\text { Expositions- } \\
\text { höhe }\end{array}$ & $\begin{array}{l}\text { Expositions- } \\
\text { dauer }\end{array}$ \\
\hline $\begin{array}{l}\text { unfallartiges Inhalations- } \\
\text { trauma (RADS) }\end{array}$ & $\begin{array}{l}\text { sehr hoch>> } \\
\text { AGW }\end{array}$ & $<1$ Tag \\
\hline $\begin{array}{l}\text { langsam entstehendes } \\
\text { irritatives Berufsasthma } \\
\text { durch Belastungen im } \\
\text { Grenzwertbereich }\end{array}$ & $\begin{array}{l}\text { grenzwertig } \\
\text { hoch, } \\
\text { AGW-Bereich }\end{array}$ & $\begin{array}{l}>1 \text { Tag bis } \\
4 \text { Monate }\end{array}$ \\
\hline $\begin{array}{l}\text { sehrlangsam entstehendes } \\
\text { irritatives Berufsasthma } \\
\text { durch untergrenzwertige } \\
\text { Belastung }\end{array}$ & niedrig, <AGW & $>4$ Monate \\
\hline
\end{tabular}

9 bis $25 \%$ der Fälle von Asthmaerkrankungen des Erwachsenen haben arbeitsbedingte Ursachen [682-685]. > Tab.13 listet Arbeitsbereiche mit besonderer Gefährdung auf. Da im Einzelfall sich die arbeitsbedingte Genese der Erkrankung meist nicht auf den ersten Blick erschließt und mehrere Komponenten einschließen kann, ist bei jeder Asthmaerkrankung mittels einer eingehenden Arbeitsanamnese zu eruieren, ob arbeitsbedingte krankheitsauslösende Noxen vorliegen. Letztere können ein Asthma induzieren oder ein vorbestehendes (arbeitsunabhängiges) Asthma wesentlich verschlimmern ( $\triangleright$ Abb. 3).

Man unterteilt in Abhängigkeit vom zugrunde liegenden Pathomechanismus das arbeitsbedingte Asthma in eine IgE-vermittelte allergische und eine irritative Form, ferner eine weitere mit unbekanntem Pathomechanismus ( $\bullet \mathbf{A b b}$. 3). Das irritative Berufsasthma lässt sich in Abhängigkeit von der Expositionshöhe und -dauer in 3 Unterformen gliedern ( $\bullet$ Tab. 14). Eine IgE-vermittelte Genese ist dann wahrscheinlich, wenn eine Latenzperiode von mindestens mehreren Wochen zwischen Expositionsbeginn und Manifestation der Erkrankung liegt und wenn die Re-Exposition gegenüber niedrigen Schadstoffkonzentrationen zum Wiederauftreten der Symptomatik führt. Allerdings werden gleichartige Verläufe auch bei bestimmten Formen des irritativen Asthmas ("not so sudden onset of irritant asthma“, "low dose irritant asthma“; > Tab. 14) und des Asthmas mit unbekanntem Pathomechanismus gefunden [686,687]; diese Formen lassen sich aus umfangreichen Berufskrankheitensta- tistiken und aus klinischen Erfahrungsberichten dokumentieren, eingehende wissenschaftliche Studien stehen hierzu aber noch aus. In der Unterform „Reactive Airways Dysfunction Syndrome" (RADS) des irritativen Berufsasthma kommt es nach meist einmaliger, oftmals unfallartiger, sehr hoher Exposition gegenüber einem irritativ wirkenden Rauch, Gas oder Dampf (z. B. Chlorgas) erstmals zu asthmatischen Beschwerden, die in Verbindung mit einer unpezifischen bronchialen Hyperreaktivität Monate oder sogar Jahre persistieren. Voraussetzung für die Entstehung eines RADS sind erhebliche Überschreitungen der Luftgrenzwerte bzw. gesundheitsbasierter Richtwerte [688, 689].

Neben dem genannten neu entstandenen Berufsasthma ist die arbeitsplatzbedingte Verschlimmerung eines vorbestehenden oder sich parallel entwickelnden nicht berufsbedingten Asthmas ein häufiges Phänomen. Arbeitsplatzbezogene Asthmatrigger wie reizende Gase, Säurenebel, Chemikaliendämpfe können bei Patienten mit einem Asthma jedweder Ätiologie vermehrt zu Beschwerden, aber auch zu zunehmenden funktionellen Einschränkungen führen. Gerade bei schweren Verlaufsformen des Asthmas im Erwachsenenalter spielen berufliche Auslöser eine wichtige und oft unterschätzte zusätzliche pathogenetische Rolle [690]. Insgesamt sind über 370 IgE-Antikörper-induzierende Allergene und 184 atemwegsreizende chemische Noxen als Verursacher des Berufsasthmas beschrieben [691]. 


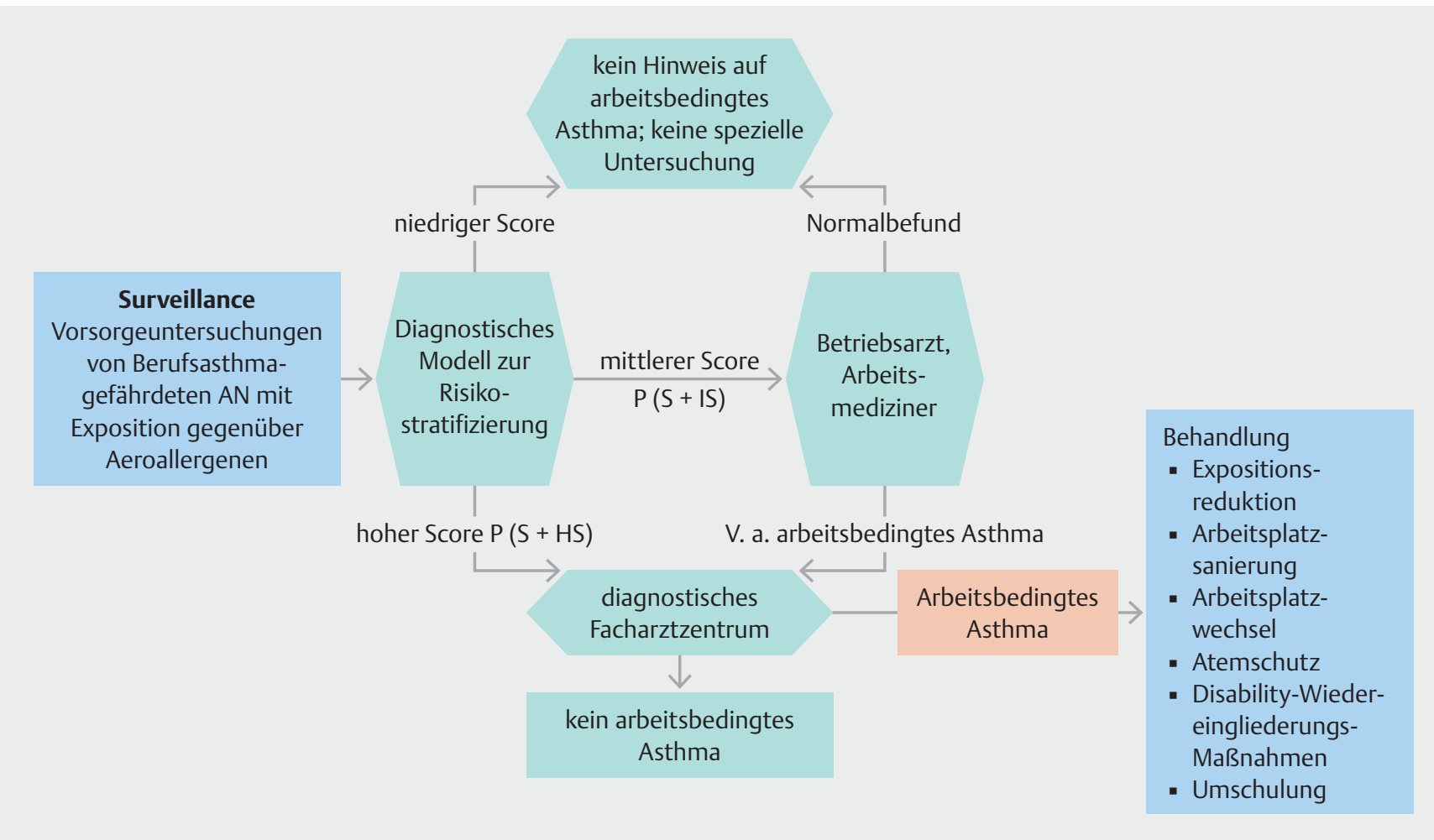

- Abb. 4 Surveillance (arbeitsmedizinische Vorsorgeuntersuchungen) von Asthma-gefährdeten Arbeitnehmern (AN) mit Stratifizierung des Procedere in Abhängigkeit vom Befund (Risiko-Score). $S$ = Prickhauttest: $P=$ positiver Befund; HS=stärker ausgeprägte Symptome; IS=mäßiggradig ausgeprägte Symptome (modifiziert nach Wilken et al. [692]).

\subsection{Arbeitsmedizinische Vorsorgen}

Personen, die aufgrund ihrer Vorgeschichte (z. B. bereits Vorliegen von Atemwegsallergien oder einer vorbestehenden unspezifischen bronchialen Hyperreagibilität) und/oder Exposition gegenüber potenziell Berufsasthma-auslösenden Noxen ein erhöhtes Krankheitsrisiko haben, sollten einer regelmäßigen Surveillance unterzogen werden ( $>$ Abb.4). Im Falle des Auftretens von Asthmasymptomen oder einer Sensibilisierung ist dann eine eingehende Diagnostik einschließlich Lungenfunktionsprüfung erforderlich (siehe unten).

\subsection{Diagnostik bei Verdacht auf Berufsasthma}

\subsubsection{Anamnese}

Die Anamnese gibt oft Hinweise auf eine arbeitsbedingte Verursachung oder Triggerung der Asthmaerkrankung. Wichtig ist, ob sich die Asthmasymptome während Wochenenden und längerer arbeitsfreier Zeiten bessern und eine Verschlechterung bei Wiederaufnahme der Tätigkeit eintritt. Bei Erkrankungen durch hochmolekulare Auslöser (pflanzliche und tierische Allergene) ist die Treffsicherheit von Schlüsselsymptomen dabei deutlich höher als bei Erkrankungen durch niedermolekulare Auslöser, insbesondere zahlreiche Chemikalien [693].
10.2.2 Spezielle Arbeitsanamnese: Tätigkeitsbeschreibung

- jetzige Tätigkeitsbeschreibung

- frühere Tätigkeitsbeschreibungen lückenlos ab Schulabgang (einschließlich Wehrdienst, nicht versicherten Zeiten/ Schwarzarbeit, Auslandseinsätzen etc.)

- für alle Zeiträume: Auflistung der Arbeitsvorgänge und -stoffe (Schemazeichnung/Fotos oft hilfreich, Nachbarschaftsexposition?)

- unfallartige Expositionen in der Vorgeschichte (z. B. bei Betriebsstörungen/Revisionen, Dämpfe, Verschütten größerer Chemikalienmengen)?

10.2.3 Weitere potenzielle Risikofaktoren

- Raucheranamnese

- allergische Rhinokonjunktivitis oder Asthma in der Vorgeschichte

- allergische Erkrankungen in der Familienanamnese

\subsubsection{Symptome}

- Art

- Rhinorrhoe, Konjunktivitis, Urtikaria

- Husten, Kurzatmigkeit, Pfeifen, Giemen

- systemische Symptome (v. a. Fieber, Schüttelfrost, Myalgien - aus differenzialdiagnostischen Überlegungen)

- örtlicher und Tätigkeitsbezug 


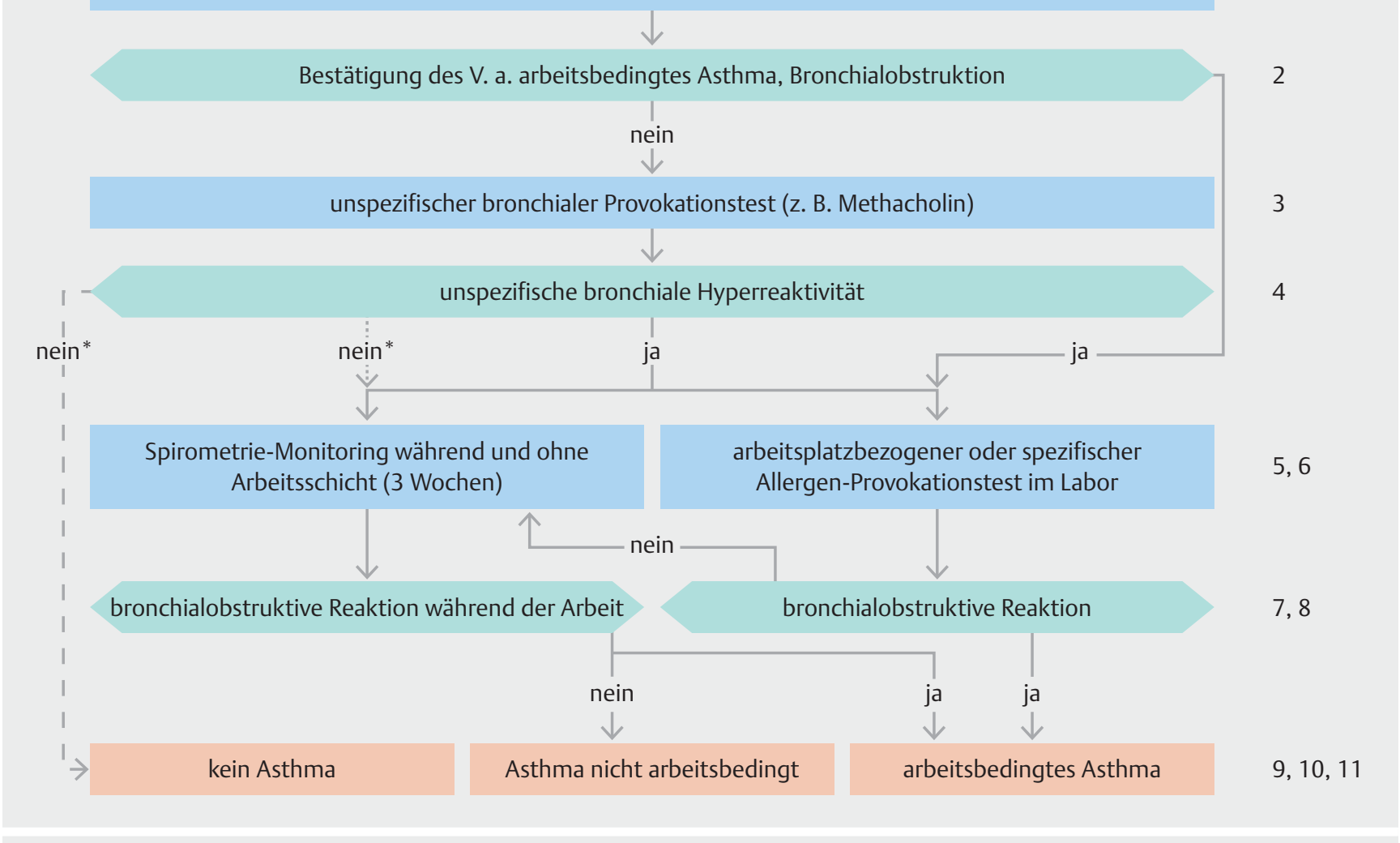

- Abb.5 Stufenschema zur Diagnostik des arbeitsbedingten Asthmas. *Im Falle der überzeugenden Schilderung von Asthmaanfällen bei der Arbeit, insbesondere bei Verdacht auf ein Isocyanat-Asthma, sollte auch bei fehlender unspezifischer Hyperreaktivität eine weitergehende Diagnostik erfolgen (cave: falsch negative Befunde).

- zeitlicher Verlauf

- Wie lange nach Beginn einer bestimmten Tätigkeit? Nach Verfahrenswechsel? Nach Wechsel eines Arbeitsstoffs?

- Beschwerdebeginn unmittelbar bei Exposition nach Arbeitsanfang?

- verzögerter Beschwerdebeginn 2-12 Stunden nach Tätigkeitsaufnahme oder erst nach Arbeitsende?

- duale asthmatische Symptome?

- Beschwerdefreiheit an arbeitsfreien Tagen, im Urlaub?

\subsubsection{Klinische Befunde und Lungenfunktionsmessungen}

Die klinischen Befunde weisen bei arbeitsbedingten Asthmaformen bis auf den Arbeitsbezug der Beschwerden und der Lungenfunktionseinschränkungen keine Besonderheiten gegenüber anderen Formen eines Asthmas auf.

Ein normaler Lungenfunktionsbefund unter Ruhebedingungen in der Arztpraxis schließt ein Berufsasthma nicht aus. Noch während der bisherigen beruflichen Tätigkeit sollte eine unspezifische bronchiale Provokationstestung (Methacholin) durchgeführt werden. Ist die Basis-Lungenfunktion normal und keine unspezifische Atemwegsempfindlichkeit (zum Zeitpunkt, an dem der Patient am Arbeitsplatz noch exponiert ist!) objektivierbar, ist in den meisten Fällen ein (arbeitsbedingtes) Asthma unwahrscheinlich. Vorsicht ist geboten bei überzeugender Angabe arbeitsplatzbezogener Asthmaanfälle, ferner beim Isocyanat-Asthma, bei welchem eine unspezifische Atemwegsüberempfindlichkeit nicht selten fehlt. In solchen unklaren Fällen ist eine weitergehende Diagnostik erforderlich ( $\bullet$ Abb.5).

Entscheidende Informationen über die ursächliche Noxe liefert oft die Dokumentation des Lungenfunktionsverlaufs. Dies erfolgt als serielles spirometrisches Monitoring vor, während und nach der als ursächlich angesehenen Arbeitsstoffexposition während Arbeitsschichten (Exposition) als auch Karenzphasen (Wochenende, Urlaub, Arbeitsunfähigkeit) über 3 Wochen. Hierbei werden von den gut eingewiesenen und kooperierenden Probanden in etwa 2-stündlichen Intervallen Lungenfunktionsmessungen mithilfe portabler elektronischer Kleinspirometer vorgenommen. 


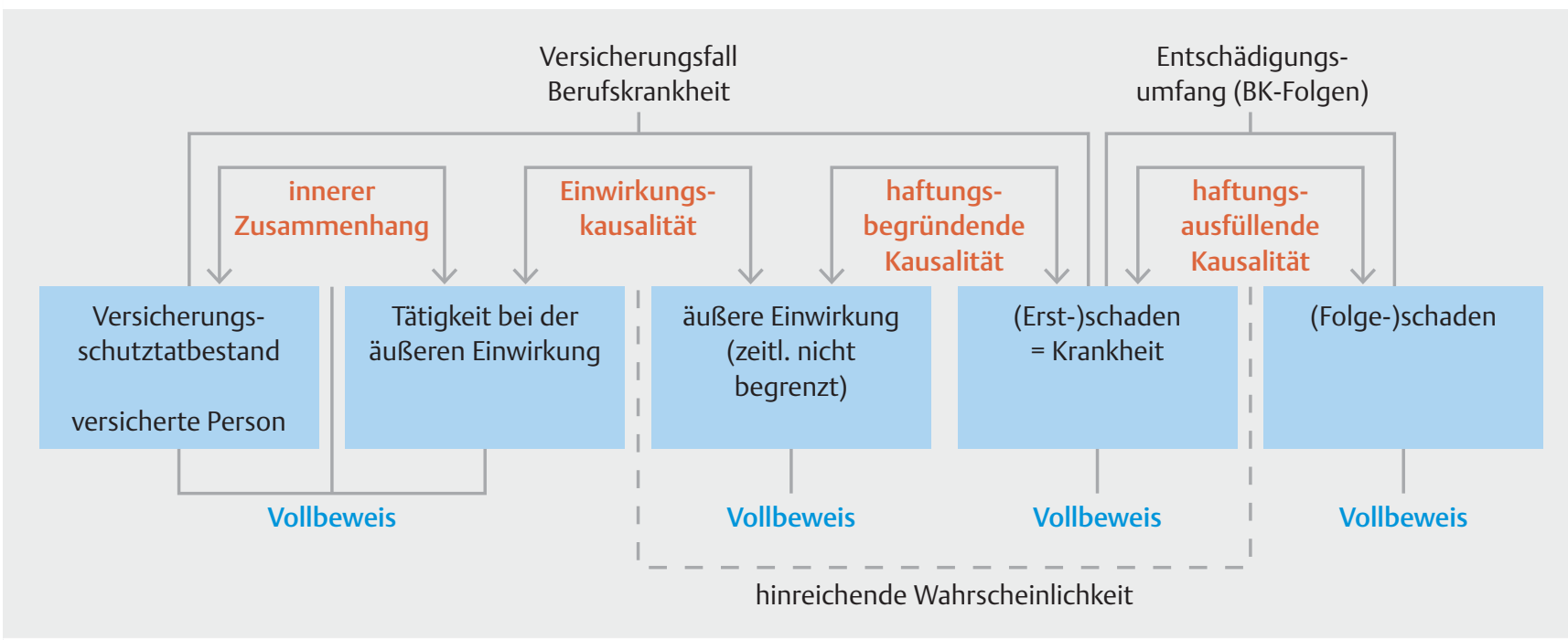

- Abb. 6 Ablauf des Begutachtungsverfahrens mit den zu beweisenden Parametern und dem als wahrscheinlich geforderten Zusammenhang zwischen gesicherter Einwirkungskausalität und der Krankheit mit ihrem Folgeschaden (Funktionsstörung).

Auch die longitudinale Bestimmung der Methacholin-Empfindlichkeit kann diagnostische Hinweise geben, insbesondere, wenn die unspezifische Atemwegsempfindlichkeit nach einer Arbeitswoche mit einem sensibilisierenden Arbeitsstoff höher ist als nach einer längeren expositionsfreien Zeit (z. B. Urlaub).

Als Goldstandard der Sicherung der Diagnose eines allergischen Asthmas gelten der spezifische inhalative Provokationstest mit Allergenextrakten und der arbeitsplatzbezogene Provokationstest mit dem als ursächlich angeschuldigten Arbeitsstoff. Entsprechendes gilt für die nicht durch hohe Expositionen ausgelösten Formen des irritativen Asthmas. Hierzu ist hohe Fachkompetenz und eingehende Erfahrung Voraussetzung; die Untersuchung ist daher dem pneumologischen oder arbeitsmedizinischen Spezialisten vorbehalten [694].

\subsubsection{Allergologische Diagnostik}

Die allergologische Vorsorgeuntersuchung von Risikopatienten und die Diagnostik von symptomatischen Arbeitnehmern beinhaltet Pricktestung mit ubiquitären und den am jeweiligen Arbeitsplatz vorliegenden speziellen beruflichen Allergenen, die meist nur in Spezialambulanzen vorgehalten werden. Die spezifische IgE-Bestimmung zum Suchen nach Sensibilisierungen gegenüber bestimmten Arbeitsstoffen ist hilfreich, aber nicht für alle in Frage kommenden Stoffe verfügbar. Die Bestimmung des Gesamt-IgE hilft nicht wesentlich weiter, denn es kann bei schmalem Sensibilisierungsspektrum normal und aufgrund anderer Erkrankungen (z. B. früherer Wurmbefall) erhöht sein und damit als „falsch negativer“ oder "falsch positiver“ Befund in die Irre führen.

\subsubsection{Entzündungsmonitoring}

Die serielle Bestimmung der NO-Konzentration in der Ausatemluft ist ein relativ neuer, vielversprechender Parameter zum Monitoring der Atemwegsentzündung, dessen Wert nicht nur zur Verlaufsbeurteilung, sondern auch zur Kausalattribution aller- dings noch in größeren Studien gezeigt werden muss. Eine Sputum-Eosinophilie kann durch hoch- wie auch teilweise durch niedermolekulare Asthmaauslöser hervorgerufen werden. Die Kombination der seriellen Lungenfunktionsuntersuchung mit dem Entzündungsmonitoring im Sputum ist geeignet, die Sensitivität der Diagnostik des arbeitsbedingten Asthmas zu erhöhen.

\subsection{Berufskrankheitenanzeige und Berufskrankheitsverfahren}

Jeder begründete Verdacht auf das Vorliegen einer Berufskrankheit, also auch eines arbeitsbedingt neu entstandenen oder wesentlich verschlimmerten vorbestehenden Asthmas, muss vom Arzt dem Unfallversicherungsträger per Meldeformular mitgeteilt werden (alternativ dem staatlichen Gewerbearzt). Formulare sind im Internet erhältlich [695]. Die Unfallversicherung ermittelt daraufhin zunächst die stattgehabte Belastung und holt ärztliche Befundberichte ein. In der Regel folgt dann die Beauftragung eines erfahrenen ärztlichen Sachverständigen zur erforderlichen ergänzenden Diagnostik (siehe > Abb.6) und Zusammenhangsbeurteilung. Die Einwirkungskausalität (unfallversicherte Person mit gefährdender Exposition/Tätigkeit) muss gegeben, das Krankheitsbild mit seinen Folgeschäden ebenfalls gesichert sein. Die Zusammenhänge zwischen der Exposition und dem Krankheitsbild und zwischen Letzterem und dem Folgeschaden müssen hinreichend wahrscheinlich sein.

Vorrangig kommen die nachfolgend angeführten Berufskrankheiten der Anlage der Berufskrankheitenverordnung (BKV) [696] in Betracht. Formale Voraussetzung für deren Anerkennung ist die Aufgabe der schädigenden Exposition bzw. Tätigkeit (nicht des Berufs). Oftmals gelingt es durch geeignete Karenzmaßnahmen im Betrieb unter Beteiligung des Unfallversicherungsträgers bereits Erkrankte an ihrem Arbeitsplatz zu halten, ohne dass eine weitere Gefährdung besteht. Rechtsgrundlage hierfür ist der $\S 3 \mathrm{BKV}$ : „Besteht für Versicherte die 
Gefahr, dass eine Berufskrankheit entsteht, wiederauflebt oder sich verschlimmert, haben die Unfallversicherungsträger dieser Gefahr mit allen geeigneten Mitteln entgegenzuwirken ...“

\subsubsection{BK Nr. 4301}

„Durch allergisierende Stoffe verursachte obstruktive Atemwegserkrankungen (einschließlich Rhinopathie), die zur Unterlassung aller Tätigkeiten gezwungen haben, die für die Entstehung, die Verschlimmerung oder das Wiederaufleben der Krankheit ursächlich waren oder sein können“.

\subsubsection{BK Nr. 4302}

„Durch chemisch-irritativ oder toxisch wirkende Stoffe verursachte obstruktive Atemwegserkrankungen, die zur Unterlassung aller Tätigkeiten gezwungen haben, die für die Entstehung, die Verschlimmerung oder das Wiederaufleben der Krankheit ursächlich waren oder sein können“.

\subsubsection{BK Nr. 1315}

„Erkrankungen durch Isocyanate, die zur Unterlassung aller Tätigkeiten gezwungen haben, die für die Entstehung, die Verschlimmerung oder das Wiederaufleben der Krankheit ursächlich waren oder sein können“.

\subsection{Verlauf, Prognose und Therapie}

Die meisten Patienten, bei denen sich ein arbeitsbedingtes Asthma entwickelt, erleben dies in den ersten ein bis zwei Jahren nach Expositionsbeginn. Die Latenzperiode kann auch deutlich länger sein. Bei einer allergischen Genese geht der Asthmamanifestation in der Regel eine Rhinokonjunktivitis voraus.

Die Prognose des Berufsasthmas ist oftmals ungünstig. Bei etwa $70 \%$ aller Patienten mit Berufsasthma persistiert die Symptomatik trotz Expositionskarenz, vielfach bleibt eine unspezifische Atemwegsüberempfindlichkeit bestehen [697]. Die medikamentöse Therapie ist nur in Verbindung mit einer konsequenten Karenz bzgl. des krankheitauslösenden Agens erfolgversprechend. Es ist wichtig, bei Verdacht auf ungünstige berufliche Einflüsse am Arbeitsplatz keine überstürzte Tätigkeitsaufgabe vorzunehmen, bevor nicht die Diagnostik sorgfältig und vollständig vorgenommen wurde.

\subsection{Prävention}

Das Schlüsselprinzip der Primärprävention des Berufsasthmas ist die Vermeidung oder zumindest wesentliche Reduktion der Exposition gegenüber Asthmaauslösern [698]. Sekundärpräventive Maßnahmen beinhalten regelmäßige arbeitsmedizinische Vorsorgen in Risikobereichen und von Risikopersonen [692], die Frühdiagnostik bei arbeitsplatzbezogenen Atembeschwerden oder Sensibilisierungen. Wenn die Erkrankung gesichert ist, ist eine im Krankheitsverlauf frühzeitige Expositionskarenz der beste Prädiktor für eine gute Prognose [698 - 700].

Im Erkrankungsfall ist die konsequente Meidung der Krankheitsauslöser die einzige erfolgversprechende Maßnahme [697]. Die Effizienz von Atemschutzgeräten ist nicht gesichert, sie sind allenfalls zur initialen und sofortigen Intervention sinnvoll.

\section{Asthma in der Schwangerschaft}

Die physiologischen Veränderungen während der Schwangerschaft können den Asthmaverlauf sowohl positiv als auch negativ beeinflussen. In etwa einem Drittel der Fälle verbessern sich die Asthmasymptome, bei einem weiteren Drittel tritt keine Änderung auf, in ebenfalls etwa einem Drittel verschlechtert sich die Symptomatik, insbesondere bei weiblichen Foeten [701 - 703]. Exazerbationen können in der Schwangerschaft gehäuft auftreten, insbesondere am Ende des 2. Trimenons [704]. Als Ursachen für Exazerbationen und eine schlechte Asthmakontrolle kommen mechanische, hormonelle und immunologische Veränderungen während der Schwangerschaft in Betracht, aber auch die Reduktion der notwendigen Asthmamedikation, insbesondere der Glucocorticosteroide, durch die Schwangere und/oder die behandelnden Ärzte infolge von Verunsicherungen über potenzielle Gefahren der Asthmamedikamente [705]. Schwangere Asthmatikerinnen zeigen sich besonders anfällig gegen über viral bedingten Atemwegsinfekten [706]. Infekte [706] und eine schlechte Asthmakontrolle können zu Komplikationen in der Schwangerschaft von Asthmatikerinnen und bezüglich der Entwicklung des Foeten führen.

EMPFEHLUNGEN

E77 Bei Frauen mit Asthma soll bei Kontrolluntersuchungen in der Schwangerschaft auch die Asthmakontrolle überprüft werden.

E78 Die Langzeittherapie und die Bedarfstherapie des Asthmas sollen während der Schwangerschaft in gewohnter Weise fortgeführt werden.

E79 Eine Therapie mit Leukotrien-Rezeptor-Antagonisten soll während der Schwangerschaft nicht begonnen werden.

E80 Eine Schwangerschaft gilt als Kontraindikation für den Beginn einer SIT. Die Fortführung der SIT mit Inhalationsallergenen während einer Schwangerschaft ist allerdings bei guter Verträglichkeit möglich (hierfür sind die entsprechenden Empfehlungen in den Fach- und Gebrauchsinformationen zu beachten).

E81 Eine schwere Asthmaexazerbation in der Schwangerschaft soll immer als stationär zu behandelnder Notfall angesehen werden. Eine zusätzliche Sauerstoffbehandlung soll unverzüglich eingeleitet werden.

E82 Bei Frauen mit Asthma soll Oxitocin als Mittel der ersten Wahl für eine etwaige Geburtseinleitung und Behandlung der postpartalen Uterusatonie verabreicht werden.

S25 Bei einer guten Kontrolle des Asthmas in der gesamten Schwangerschaft ist die perinatale Prognose der Kinder denen von nicht asthmatischen Müttern vergleichbar. 
So finden sich bei Schwangeren mit Asthma höhere Raten an Präeklampsie, Schwangerschaftsdiabetes, Plazentaablösung, Plazenta prävia und Frühgeburten [707].

Ein unkontrolliertes Asthma ist auch für den Föten mit einem höheren Risiko an Komplikationen verbunden, wie intrauteriner Wachstumshemmung, vaginalen Blutungen, Frühgeburt und Hypoxie des Neugeborenen [704, 708]. Wenn das Asthma während der Schwangerschaft gut kontrolliert ist, ist das Risiko mütterlicher und fetaler Komplikationen gegenüber Schwangeren ohne Asthma nicht oder allenfalls gering erhöht [709]. Daher kommt einer guten Asthmakontrolle in der Schwangerschaft zur Vermeidung von Exazerbationen und Komplikationen große Bedeutung zu.

\subsection{Beratung}

Asthmatikerinnen sollte zu Beginn einer Schwangerschaft ein Beratungsgespräch zur Bedeutung und Sicherheit der während der Schwangerschaft fortzuführenden Asthmatherapie mit dem Ziel einer guten Asthmakontrolle angeboten werden.

Chronischer Zigarettenkonsum führt bei rauchenden Schwangeren zu ungünstigen Auswirkungen auf den Foeten bzw. das Kind: Reduktion der neonatalen Immunfunktion, erhöhtes Risiko für den plötzlichen Kindstod, niedriges Geburtsgewicht, Frühgeburt, Lungenfunktionseinschränkungen im Schulalter, höhere Rate an Atemwegsinfektionen, psychische Auffälligkeiten und Asthma. Zudem ist die Gefahr größer für eine Plazentaablösung, eine Plazenta prävia und Uterusblutungen [710,711]. Der Nutzen einer Raucherentwöhnung steht daher für die werdende Mutter und das werdende Kind außer Frage [712].

\subsection{Kontrolluntersuchungen}

Schwangere Asthmatikerinnen sollten engmaschig kontrolliert werden, um bei jeder Verlaufsänderung die Therapie angemessen anpassen zu können. Zur Ermittlung der Asthmakontrolle können neben Anamnese, klinischer Untersuchung, Kenngrößen der Lungenfunktion unter Einschluss der Blutgasanalyse auch ein Fragebogen zur Güte der Asthmakontrolle [713] und die exspiratorische NO-Konzentration eingesetzt werden [169]. Auch die Überprüfung der Asthmamedikation durch kompetente Apotheker kann hilfreich sein [714].

Bei schwerem Asthma wird eine engmaschige Überwachung auch des Ungeborenen empfohlen. Frauen mit schlecht einstellbarem Asthma sollten vom Pneumologen und Gynäkologen gemeinsam in enger Abstimmung betreut werden.

\subsection{Pharmakotherapie}

Ein unkontrolliertes Asthma schadet der Schwangeren und dem ungeborenen Kind. Die Gefahren einer unzureichenden Asthmakontrolle überwiegen bei weitem befürchtete Medikamentennebenwirkungen, die eher überschätzt werden und zu unzureichender Therapieadhärenz Anlass geben.

Für die Asthmabehandlung gelten folgende allgemeine Empfehlungen:
- optimierte Patientenbetreuung durch Pneumologen und Gynäkologen

- strukturierte Patientenschulung [715]

- leitliniengerechte Asthmatherapie mit wenigen Ausnahmen

- Da der Fötus in einer Umgebung niedriger Sauerstoffpartialdrücke aufwächst, stellt der Asthmaanfall sowohl für die Schwangere als auch für das ungeborene Kind einen Notfall dar und ist im Krankenhaus unter fetalem Monitoring zu behandeln. Im Anfall ist eine (meist nasale) Sauerstoffgabe zur Vermeidung einer Hypoxämie obligat.

Die Basistherapie der Asthmatikerin sollte nicht wegen des Eintritts einer Schwangerschaft geändert werden. Der Einsatz von inhalativen Glucocorticosteroiden (ICS), Beta-2-Sympathomimetika, Montelukast oder Theophyllin ist nicht mit einer erhöhten Inzidenz von foetalen Abnormitäten assoziiert [716].

Die Inhalation von ICS, von denen für Budesonid die größten Erfahrungen in der Schwangerschaft vorliegen [717], beugt Asthmaexazerbationen in der Schwangerschaft vor [709, 718, 719]; das Absetzen von ICS während der Schwangerschaft ist hingegen ein signifikanter Risikofaktor für das Auftreten von Exazerbationen [704]. Bei Abwägung der Evidenz für ungünstige Auswirkungen von Exazerbationen und der Sicherheit üblicher Dosierungen von ICS und LABA sollte die Asthmabehandlung während der Schwangerschaft besser nicht mit einer Dosisreduktion dieser Substanzen einhergehen [716].

Der Einsatz von monoklonalen Antikörpern kann aufgrund fehlender Daten während der Schwangerschaft nicht mit hinreichender Validität bewertet werden. Es wird empfohlen, auf den Einsatz während der Schwangerschaft zu verzichten, außer bei Patientinnen, bei denen diese Therapie eindeutig erforderlich ist [720].

\subsection{Spezifische Immuntherapie}

Zur Immuntherapie während der Schwangerschaft gibt es keine verlässlichen Daten. Eine spezifische Immuntherapie (SIT) sollte aufgrund des möglichen Auftretens von systemischen Reaktionen nicht während der Schwangerschaft begonnen, kann aber bei guter Verträglichkeit und bei bisher problemlosem Verlauf der SIT während der Schwangerschaft fortgesetzt werden.

\subsection{Schutzimpfungen}

Wegen unzureichender Daten bezüglich des Einsatzes der Influenza- und Pneumokokkenschutzimpfung während der Schwangerschaft von Asthmatikerinnen sollten diese Impfungen möglichst vor Beginn einer Schwangerschaft durchgeführt werden.

\subsection{Behandlung von Atemwegsinfekten}

Pulmonale Infekte sind bei asthmakranken Schwangeren häufig. Bei den meist viralen Infekten ist der Einsatz von Antibiotika nicht erforderlich. Da durch den Einsatz von Antibiotika das Risiko eines Asthmas für die Nachkommen möglicherweise erhöht ist [721], sollte die Gabe von Antibiotika auf die notwendigen Indikationen beschränkt werden.

Vorgeschlagen werden Penicilline und Cephalosporine für den Einsatz während der Schwangerschaft, wenn keine Allergien oder Unverträglichkeiten gegenüber diesen Substanzen 
bekannt sind [721]. Erythromycin erscheint sicher, kann aber gastrointestinale Nebenwirkungen, insbesondere Übelkeit hervorrufen. Aminoglykoside sollten wegen ihrer Oto- und Nephrotoxizität, Tetrazykline wegen ihrer wachstumshemmenden Effekte sowie Chinolone wegen der Auslösung von Knochendefekten vermieden werden.

\subsection{Behandlung des Asthmaanfalls}

Die medikamentöse Therapie des Asthmaanfalls in der Schwangerschaft sollte wie bei nicht schwangeren Asthmatikerinnen erfolgen, eine Untertherapie aus Furcht vor unerwünschten Effekten der Medikation ist zu vermeiden [704]. Ein schwerer Asthmaanfall in der Schwangerschaft soll immer als stationär zu behandelnder Notfall - in der zweiten Schwangerschaftshälfte unter Konsultation eines Gynäkologen - angesehen werden. Um eine Hypoxie des Foeten zu vermeiden, ist der frühzeitige Einsatz von SABA, systemischen Glucocorticosteroiden und von $\mathrm{O}_{2}$ zu empfehlen mit dem Ziel, eine $\mathrm{O}_{2}$-Sättigung von mehr als $95 \%$ und einen $\mathrm{PaCO}_{2}$ von weniger als $35 \mathrm{mmHg}$ zur Verbesserung der foetalen $\mathrm{O}_{2}$-Utilisation zu erreichen.

In schweren Fällen können bei entsprechender Indikation inhalativ Ipratropiumbromid und intravenös Beta-2-Sympathomimetika eingesetzt werden.

\subsection{Management während der Geburt}

Asthmaanfälle während der Entbindung sind selten. Eine während der Entbindung auftretende Hyperventilation kann zu einer Bronchokonstriktion führen, die durch Inhalation von SABA behandelt werden kann. Falls die Asthmatikerin präpartal mit mehr als $7,5 \mathrm{mg}$ Prednisolon/Tag über einen Zeitraum von mehr als 2 Wochen behandelt wurde, ist bei der Entbindung die parenterale Applikation von $100 \mathrm{mg}$ Hydrocortison in 6bis 8-stündigen Abständen zu empfehlen. Falls eine Narkose erforderlich ist, ist eine Regionalanästhesie gegenüber einer Vollnarkose zu bevorzugen. Empfehlenswert zur Anästhesie sind Fentanyl, zur Narkose Propofol und Ketamin.

Zur intrapartalen Wehenhemmung können Beta-2-Sympathomimetika wie bei Schwangeren ohne Asthma eingesetzt werden. Für die Behandlung einer vorzeitigen Wehentätigkeit kann der Oxytocinantagonist Atosiban aufgrund geringer Nebenwirkungen auch bei Schwangeren mit Asthma eingesetzt werden.

Falls hohe Dosen von SABA während der letzten 48 Stunden vor der Entbindung eingesetzt wurden, kann eine neonatale Hypoglykämie auftreten, insbesondere bei Frühgeborenen. Daher sollte bei diesen Babies innerhalb der ersten 24 Stunden nach der Geburt der Blutzucker kontrolliert werden [722].

\subsection{Geburtseinleitung bei Asthma}

Bei einer Geburtseinleitung mit Prostaglandinen ist ihre bronchokonstriktorische Wirkung zu berücksichtigen. In der medikamentösen Therapie der postpartalen Uterusatonie ist Oxytocin das Medikament der ersten Wahl. Prostaglandin F2a und Sulproston sind bei Asthma kontraindiziert.

\subsection{Stillzeit}

Die zur Behandlung von Asthma angewandten Medikamente können auch bei stillenden Müttern eingesetzt werden. Weniger als $1 \%$ der mütterlichen Theophyllin-Dosis geht in die Muttermilch über. Bei Prednisolon betragen die Konzentrationen in der Muttermilch nur 5-25\% der Serumkonzentration. Der in der Muttermilch gefundene Anteil einer intravenösen oder oralen Prednisolongabe beträgt weniger als $0,1 \%$. Damit wird der gestillte Säugling nur minimalen Steroidmengen und damit keinem klinisch bedeutsamen Risiko ausgesetzt.

\section{Interessenkonflikt}

Eine Übersicht der Interessenkonflikte findet sich im Internet unter http://www.awmf.org; AWMF-Registernummer 020-009.

\section{Literatur}

[1] Buhl R, Berdel D, Criée CP et al. [Guidelines for diagnosis and treatment of asthma patients]. Pneumologie 2006; 60: 139-177

[2] Guideline (GINA) Global Initiative for Asthma Management and Prevention. Verfügbar unter www.ginasthma.com

[3] www.awmf.org

[4] Holgate ST. Pathogenesis of asthma. Clin Exp Allergy 2008; 38: 872 897

[5] Holgate ST, Davies DE. Rethinking the pathogenesis of asthma. 2009; 31: $362-367$

[6] An SS, Bai TR, Bates JH et al. Airway smooth muscle dynamics: a common pathway of airway obstruction in asthma. Eur Respir J 2007; 29: $834-860$

[7] Wang L, McParland BE, Paré PD. The functional consequences of structural changes in the airways: implications for airway hyperresponsiveness in asthma. Chest 2003; 123: 356S-362S

[8] Holgate ST. The airway epithelium is central to the pathogenesis of asthma. Allergol Int 2008; 57: 1-10

[9] Holt PG, Sly PD. Environmental Microbial Exposure and Protection against Asthma. N Engl J Med 2015; 373: 2576 - 2578

[10] Holgate ST, Davies DE, Lackie PM et al. Epithelial-mesenchymal interactions in the pathogenesis of asthma. J Allergy Clin Immunol 2000; 105: $193-204$

[11] Lambrecht BN, Hammad H. Biology of lung dendritic cells at the origin of asthma. Immunity 2009; 31: 412-424

[12] Bratke K, Lommatzsch M, Julius P et al. Dendritic cell subsets in human bronchoalveolar lavage fluid after segmental allergen challenge. Thorax 2007; 62: 168-175

[13] Robinson DS. The role of the T cell in asthma. J Allergy Clin Immunol 2010; 126: 1081-1091

[14] Smith SG, Chen R, Kjarsgaard M et al. Increased numbers of activated group 2 innate lymphoid cells in the airways of patients with severe asthma and persistent airway eosinophilia. J Allergy Clin Immunol 2016; 137: $75-86$.e8

[15] Lambrecht BN, Hammad H. The immunology of asthma. Nat Immuno 2015; 16: $45-56$

[16] Bradding P, Walls AF, Holgate ST. The role of the mast cell in the pathophysiology of asthma. J Allergy Clin Immunol 2006; 117: 1277 1284 
[17] Bochner BS, Gleich G]. What targeting eosinophils has taught us about their role in diseases. J Allergy Clin Immunol 2010; 126: 16- 25

[18] Price DB, Rigazio A, Campbell JD et al. Blood eosinophil count and prospective annual asthma disease burden: a UK cohort study. Lancet Respir Med 2015; 3: 849-858

[19] Fahy JV. Eosinophilic and neutrophilic inflammation in asthma: insights from clinical studies. Proc Am Thorac Soc 2009; 6: 256-259

[20] Kornerup KN, Page CP. The role of platelets in the pathophysiology of asthma. Platelets 2007; 18: 319-328

[21] Lommatzsch M, Schloetcke K, Klotz ] et al. Brain-derived neurotrophic factor in platelets and airflow limitation in asthma. Am J Respir Crit Care Med 2005; 171: $115-120$

[22] Lommatzsch M, Lindner $Y$, Edner A et al. Adverse effects of salmeterol in asthma: a neuronal perspective. Thorax 2009; 64: 763 - 769

[23] Benton AS, Kumar N, Lerner ] et al. Airway platelet activation is associated with airway eosinophilic inflammation in asthma. J Investig Med 2010; 58: 987 - 990

[24] Undem B], Carr MJ. The role of nerves in asthma. Curr Allergy Asthma Rep 2002; 2: 159 - 165

[25] Verhein KC, Fryer AD, Jacoby DB. Neural control of airway inflammation. Curr Allergy Asthma Rep 2009; 9: 484-490

[26] Fryer AD, Stein LH, Nie $Z$ et al. Neuronal eotaxin and the effects of CCR3 antagonist on airway hyperreactivity and $\mathrm{M} 2$ receptor dysfunction. J Clin Invest 2006; 116: 228-236

[27] Zanini A, Chetta A, Imperatori AS et al. The role of the bronchial microvasculature in the airway remodelling in asthma and COPD. Respir Res 2010; 11: 132

[28] Detoraki A, Granata F, Staibano S et al. Angiogenesis and lymphangiogenesis in bronchial asthma. Allergy 2010; 65: 946-958

[29] Murphy DM, O'Byrne PM. Recent advances in the pathophysiology of asthma. Chest 2010; 137: 1417-1426

[30] Meyers DA. Genetics of asthma and allergy: what have we learned? J Allergy Clin Immunol 2010; 126: 439-446

[31] Beuther DA. Obesity and asthma. Clin Chest Med 2009; 30: 479 - 488

[32] Thyagarajan B, Jacobs DR, Apostol GG et al. Longitudinal association of body mass index with lung function: the CARDIA study. Respir Res 2008; 9: 31

[33] Haldar P, Pavord ID, Shaw DE et al. Cluster analysis and clinical asthma phenotypes. Am J Respir Crit Care Med 2008; 178: 218-224

[34] Torchio R, Gobbi A, Gulotta C et al. Mechanical effects of obesity on airway responsiveness in otherwise healthy humans. J Appl Physiol 2009; 107: $408-416$

[35] Bisgaard H, Bønnelykke K. Long-term studies of the natural history of asthma in childhood. J Allergy Clin Immunol 2010; 126: 187-197

[36] Hasler G, Gergen PJ, Kleinbaum DG et al. Asthma and panic in young adults: a 20-year prospective community study. Am J Respir Crit Care Med 2005; 171: $1224-1230$

[37] Scott KM, Von Korff M, Alonso J et al. Childhood adversity, early-onset depressive/anxiety disorders, and adult-onset asthma. Psychosom Med 2008; 70: 1035-1043

[38] Chen E, Chim LS, Strunk RC et al. The role of the social environment in children and adolescents with asthma. Am J Respir Crit Care Med 2007; 176: 644-649

[39] Kullowatz A, Rosenfield D, Dahme B et al. Stress effects on lung function in asthma are mediated by changes in airway inflammation. Psychosom Med 2008; 70: 468-475

[40] Ritz T, Kullowatz A, Goldman MD et al. Airway response to emotional stimuli in asthma: the role of the cholinergic pathway. J Appl Physiol 2010; 108: $1542-1549$

[41] Liangas G, Morton JR, Henry RL. Mirth-triggered asthma: is laughter really the best medicine? Pediatr Pulmonol 2003; 36: 107-112
[42] Ritz T, Wittchen HU, Klotsche J et al. Asthma Trigger Reports Are Associated with Low Quality of Life, Exacerbations, and Emergency Treatments. Ann Am Thorac Soc 2016; 13: 204-211

[43] Vázquez I, Romero-Frais E, Blanco-Aparicio M et al. Psychological and self-management factors in near-fatal asthma. J Psychosom Res 2010; 68: $175-181$

[44] Andersson NW, Hansen MV, Larsen AD et al. Prenatal maternal stress and atopic diseases in the child: a systematic review of observational human studies. Allergy 2016; 71: 15-26

[45] Sears MR, Greene JM, Willan AR et al. A longitudinal, populationbased, cohort study of childhood asthma followed to adulthood. N Engl ] Med 2003; 349: 1414-1422

[46] Holt PG, Sly PD. Viral infections and atopy in asthma pathogenesis: new rationales for asthma prevention and treatment. Nat Med 2012; 18: $726-735$

[47] Platts-Mills T, Vaughan J, Squillace $S$ et al. Sensitisation, asthma, and a modified Th2 response in children exposed to cat allergen: a population-based cross-sectional study. Lancet 2001; 357: 752 - 756

[48] Lau S, Illi S, Platts-Mills TA et al. Longitudinal study on the relationship between cat allergen and endotoxin exposure, sensitization, catspecific $\mathrm{lgG}$ and development of asthma in childhood-report of the German Multicentre Allergy Study (MAS 90). Allergy 2005; 60: 766 773

[49] Illi S, von Mutius E, Lau S et al. Perennial allergen sensitisation early in life and chronic asthma in children: a birth cohort study. Lancet 2006; 368: $763-770$

[50] Deifl S, Bohle B. Factors influencing the allergenicity and adjuvanticity of allergens. Immunotherapy 2011; 3: 881-893

[51] Schaub B, Lauener R, von Mutius E. The many faces of the hygiene hypothesis. J Allergy Clin Immunol 2006; 117: 969-977

[52] Patel MM, Miller RL. Air pollution and childhood asthma: recent advances and future directions. Curr Opin Pediatr 2009; 21: 235-242

[53] Stein MM, Hrusch CL, Gozdz J et al. Innate Immunity and Asthma Risk in Amish and Hutterite Farm Children. N Engl J Med 2016; 375: 411 421

[54] Chatila TA. Innate Immunity in Asthma. N Engl J Med 2016; 375: 477 479

[55] von Mutius E, Vercelli D. Farm living: effects on childhood asthma and allergy. Nat Rev Immunol 2010; 10: $861-868$

[56] Ege M], Mayer M, Normand AC et al. Exposure to environmental microorganisms and childhood asthma. N Engl J Med 2011; 364: $701-709$

[57] Ball TM, Castro-Rodriguez JA, Griffith KA et al. Siblings, day-care attendance, and the risk of asthma and wheezing during childhood. N Engl J Med 2000; 343: 538 - 543

[58] Bønnelykke K, Vissing NH, Sevelsted A et al. Association between respiratory infections in early life and later asthma is independent of virus type. J Allergy Clin Immunol 2015; 136: 81 - 86

[59] Jackson DJ, Gangnon RE, Evans MD et al. Wheezing rhinovirus illnesses in early life predict asthma development in high-risk children. Am J Respir Crit Care Med 2008; 178: 667-672

[60] Jackson DJ, Evans MD, Gangnon RE et al. Evidence for a causal relationship between allergic sensitization and rhinovirus wheezing in early life. Am J Respir Crit Care Med 2012; 185: 281-285

[61] Tesse R, Pandey RC, Kabesch M. Genetic variations in toll-like receptor pathway genes influence asthma and atopy. Allergy 2011; 66: 307 316

[62] Huang Y], Nelson CE, Brodie EL et al. Airway microbiota and bronchial hyperresponsiveness in patients with suboptimally controlled asthma. J Allergy Clin Immunol 2011; 127: 372-381

[63] Rosen C]. Clinical practice. Vitamin D insufficiency. N Engl J Med 2011; 364: $248-254$ 
[64] Sutherland ER, Goleva E, Jackson LP et al. Vitamin D levels, lung function, and steroid response in adult asthma. Am J Respir Crit Care Med 2010; 181: 699-704

[65] Searing DA, Zhang Y, Murphy JR et al. Decreased serum vitamin D levels in children with asthma are associated with increased corticosteroid use. J Allergy Clin Immunol 2010; 125: 995 - 1000

[66] Ginde AA, Sutherland ER. Vitamin D in asthma: panacea or true promise? J Allergy Clin Immunol 2010; 126: 59-60

[67] Castro M, King TS, Kunselman SJ et al. Effect of vitamin D3 on asthma treatment failures in adults with symptomatic asthma and lower vitamin D levels: the VIDA randomized clinical trial. JAMA 2014; 311 : 2083-2091

[68] von Mutius E, Martinez FD. Inconclusive Results of Randomized Trials of Prenatal Vitamin D for Asthma Prevention in Offspring: Curbing the Enthusiasm. JAMA 2016; 315: 347-348

[69] Thomson NC, Chaudhuri R, Livingston E. Asthma and cigarette smoking. Eur Respir J 2004; 24: $822-833$

[70] Bateman ED, Boushey HA, Bousquet J et al. Can guideline-defined asthma control be achieved? The Gaining Optimal Asthma ControL study Am J Respir Crit Care Med 2004; 170: 836 - 844

[71] Chaudhuri R, Livingston E, McMahon AD et al. Cigarette smoking impairs the therapeutic response to oral corticosteroids in chronic asthma. Am J Respir Crit Care Med 2003; 168: 1308 - 1311

[72] Lazarus SC, Chinchilli VM, Rollings NJ et al. Smoking affects response to inhaled corticosteroids or leukotriene receptor antagonists in asthma. Am J Respir Crit Care Med 2007; 175: 783-790

[73] Chalmers GW, Macleod KJ, Little SA et al. Influence of cigarette smoking on inhaled corticosteroid treatment in mild asthma. Thorax 2002; $57: 226-230$

[74] Dezateux C, Stocks J, Dundas I et al. Impaired airway function and wheezing in infancy: the influence of maternal smoking and a genetic predisposition to asthma. Am J Respir Crit Care Med 1999; 159: 403 410

[75] Carlsen KH, Carlsen KC. Respiratory effects of tobacco smoking on infants and young children. Paediatr Respir Rev 2008; 9: 11-19

[76] Nurmatov U, Devereux G, Sheikh A. Nutrients and foods for the primary prevention of asthma and allergy: systematic review and metaanalysis. J Allergy Clin Immunol 2011; 127: 724-733

[77] Ma J, Strub P, Lv N et al. Pilot randomised trial of a healthy eating behavioural intervention in uncontrolled asthma. Eur Respir J 2016; 47: $122-132$

[78] Willers SM, Devereux G, Craig LC et al. Maternal food consumption during pregnancy and asthma, respiratory and atopic symptoms in 5-year-old children. Thorax 2007; 62: 773-779

[79] Bisgaard H, Stokholm J, Chawes BL et al. Fish Oil-Derived Fatty Acids in Pregnancy and Wheeze and Asthma in Offspring. N Engl J Med 2016; 375: $2530-2539$

[80] Duncan JM, Sears MR. Breastfeeding and allergies: time for a change in paradigm? Curr Opin Allergy Clin Immunol 2008; 8: 398-405

[81] Penders ], Kummeling I, Thijs C. Infant antibiotic use and wheeze and asthma risk: a systematic review and meta-analysis. Eur Respir J 2011; 38: $295-302$

[82] Wickens K, Beasley R, Town I et al. The effects of early and late paracetamol exposure on asthma and atopy: a birth cohort. Clin Exp Allergy 2011; 41: 399-406

[83] Sheehan W], Mauger DT, Paul IM et al. Acetaminophen versus Ibuprofen in Young Children with Mild Persistent Asthma. N Engl J Med 2016; 375: 619-630

[84] Kowalski ML, Makowska JS, Blanca M et al. Hypersensitivity to nonsteroidal anti-inflammatory drugs (NSAIDs) - classification, diagnosis and management: review of the EAACI/ENDA(\#) and GA2LEN/HANNA*. Allergy 2011; 66: 818-829
[85] Wenzel SE. Asthma: defining of the persistent adult phenotypes. Lancet 2006; 368: 804-813

[86] Agusti A, Bel E, Thomas M et al. Treatable traits: toward precision medicine of chronic airway diseases. Eur Respir J 2016; 47: 410 - 419

[87] Burrows B, Martinez FD, Halonen M et al. Association of asthma with serum IgE levels and skin-test reactivity to allergens. N Engl J Med 1989; 320: $271-277$

[88] Tomassen P, Jarvis D, Newson R et al. Staphylococcus aureus enterotoxin-specific IgE is associated with asthma in the general population: a GA(2)LEN study. Allergy 2013; 68: 1289-1297

[89] O'Hollaren MT, Yunginger JW, Offord KP et al. Exposure to an aeroallergen as a possible precipitating factor in respiratory arrest in young patients with asthma. N Engl J Med 1991; 324: 359-363

[90] Harju T, Keistinen T, Tuuponen T et al. Seasonal variation in childhood asthma hospitalisations in Finland, 1972-1992. Eur J Pediatr 1997; 156: $436-439$

[91] Mitakakis TZ, Tovey ER, Xuan W et al. Personal exposure to allergenic pollen and mould spores in inland New South Wales, Australia. Clin Exp Allergy 2000; 30: $1733-1739$

[92] Bousquet ], Khaltaev N, Cruz AA et al. Allergic Rhinitis and its Impact on Asthma (ARIA) 2008 update (in collaboration with the World Health Organization, GA(2)LEN and AllerGen). Allergy 2008; 63: 8160

[93] Chipps BE, Zeiger RS, Borish L et al. Key findings and clinical implications from The Epidemiology and Natural History of Asthma: Outcomes and Treatment Regimens (TENOR) study. J Allergy Clin Immunol 2012; 130: $332-342$

[94] Lommatzsch M, Korn S, Buhl R et al. Against all odds: anti-IgE for intrinsic asthma? Thorax 2014; 69: 94-96

[95] Humbert M, Menz G, Ying S et al. The immunopathology of extrinsic (atopic) and intrinsic (non-atopic) asthma: more similarities than differences. Immunol Today 1999; 20: 528 - 533

[96] Arbes SJ. Do all asthmatics with atopy have atopic asthma? J Allergy Clin Immunol 2012; 130: 1202-1204

[97] Virchow JC, Backer V, Kuna P et al. Efficacy of a House Dust Mite Sublingual Allergen Immunotherapy Tablet in Adults With Allergic Asthma: A Randomized Clinical Trial. JAMA 2016; 315: 1715- 1725

[98] Ingram JL, Kraft M. IL-13 in asthma and allergic disease: asthma phenotypes and targeted therapies. J Allergy Clin Immunol 2012; 130: $829-884$

[99] Gauthier M, Ray A, Wenzel SE. Evolving Concepts of Asthma. Am J Respir Crit Care Med 2015; 192: 660 - 668

[100] Berry M, Morgan A, Shaw DE et al. Pathological features and inhaled corticosteroid response of eosinophilic and non-eosinophilic asthma. Thorax 2007; 62: $1043-1049$

[101] Pavord ID, Korn S, Howarth P et al. Mepolizumab for severe eosinophilic asthma (DREAM): a multicentre, double-blind, placebo-controlled trial. Lancet 2012; 380: 651 - 659

[102] McGrath KW, Icitovic N, Boushey HA et al. A large subgroup of mildto-moderate asthma is persistently noneosinophilic. Am J Respir Crit Care Med 2012; 185: 612-619

[103] Fleming L, Tsartsali L, Wilson $\mathrm{N}$ et al. Sputum inflammatory phenotypes are not stable in children with asthma. Thorax 2012; 67: 675681

[104] Brusselle GG, Maes T, Bracke KR. Eosinophils in the spotlight: Eosinophilic airway inflammation in nonallergic asthma. Nat Med 2013; 19: $977-979$

[105] Hanania NA, Wenzel S, Rosén K et al. Exploring the effects of omalizumab in allergic asthma: an analysis of biomarkers in the EXTRA study. Am J Respir Crit Care Med 2013; 187: 804-811

[106] Wenzel S, Ford L, Pearlman D et al. Dupilumab in persistent asthma with elevated eosinophil levels. N Engl J Med 2013; 368: 2455 - 2466 
[107] Katz LE, Gleich G], Hartley BF et al. Blood eosinophil count is a useful biomarker to identify patients with severe eosinophilic asthma. Ann Am Thorac Soc 2014; 11: $531-536$

[108] Ortega HG, Liu MC, Pavord ID et al. Mepolizumab treatment in patients with severe eosinophilic asthma. N Engl J Med 2014; 371: $1198-1207$

[109] Castro M, Zangrilli ], Wechsler ME et al. Reslizumab for inadequately controlled asthma with elevated blood eosinophil counts: results from two multicentre, parallel, double-blind, randomised, placebocontrolled, phase 3 trials. Lancet Respir Med 2015; 3: 355-366

[110] Castro M, Wenzel SE, Bleecker ER et al. Benralizumab, an anti-interleukin 5 receptor $\alpha$ monoclonal antibody, versus placebo for uncontrolled eosinophilic asthma: a phase $2 \mathrm{~b}$ randomised dose-ranging study. Lancet Respir Med 2014; 2: 879-890

[111] Dicpinigaitis PV. Chronic cough due to asthma: ACCP evidencebased clinical practice guidelines. Chest 2006; 129: 75S-79S

[112] Morice AH, Millqvist E, Belvisi MG et al. Expert opinion on the cough hypersensitivity syndrome in respiratory medicine. Eur Respir ] 2014; 44: $1132-1148$

[113] Kardos P, Berck H, Fuchs KH et al. Guidelines of the German Respiratory Society for diagnosis and treatment of adults suffering from acute or chronic cough. Pneumologie 2010; 64: 701-711

[114] Rajan JP, Wineinger NE, Stevenson DD et al. Prevalence of aspirinexacerbated respiratory disease among asthmatic patients: A metaanalysis of the literature. J Allergy Clin Immunol 2015; 135: 676 681

[115] Farooque SP, Lee TH. Aspirin-sensitive respiratory disease. Annu Rev Physiol 2009; 71: 465-487

[116] Randolph C. An update on exercise-induced bronchoconstriction with and without asthma. Curr Allergy Asthma Rep 2009; 9: 433 438

[117] Carlsen KH. The breathless adolescent asthmatic athlete. Eur Respir ] 2011; 38: $713-720$

[118] www.nada.de

[119] www.nada.at

[120] www.antidoping.ch

[121] Lamprecht B, McBurnie MA, Vollmer WM et al. COPD in never smokers: results from the population-based burden of obstructive lung disease study. Chest 2011; 139: $752-763$

[122] Criée CP, Baur X, Berdel D et al. [Standardization of spirometry: 2015 update. Published by German Atemwegsliga, German Respiratory Society and German Society of Occupational and Environmental Medicine]. Pneumologie 2015; 69: 147-164

[123] Reed CE. Asthma in the elderly: diagnosis and management. J Allergy Clin Immunol 2010; 126: 681-687

[124] Fairs A, Agbetile J, Hargadon B et al. IgE sensitization to Aspergillus fumigatus is associated with reduced lung function in asthma. Am J Respir Crit Care Med 2010; 182: $1362-1368$

[125] Knutsen AP, Bush RK, Demain JG et al. Fungi and allergic lower respiratory tract diseases. J Allergy Clin Immunol 2012; 129: 280 - 291

[126] Ogawa Y, Calhoun W]. Phenotypic characterization of severe asthma. Curr Opin Pulm Med 2010; 16: 48 - 54

[127] Lommatzsch M, Virchow JC. Severe asthma: definition, diagnosis and treatment. Dtsch Arztebl Int 2014; 111: 847 - 855

[128] Chung KF, Wenzel SE, Brozek JL et al. International ERS/ATS guidelines on definition, evaluation and treatment of severe asthma. Eur Respir J 2014; 43: 343-373

[129] Sembajwe G, Cifuentes M, Tak SW et al. National income, self-reported wheezing and asthma diagnosis from the World Health Survey. Eur Respir J 2010; 35: 279-286
[130] von Mutius E, Martinez FD, Fritzsch C et al. Prevalence of asthma and atopy in two areas of West and East Germany. Am J Respir Crit Care Med 1994; 149: 358 - 364

[131] Nowak D, Heinrich J, Jörres R et al. Prevalence of respiratory symptoms, bronchial hyperresponsiveness and atopy among adults: west and east Germany. Eur Respir J 1996; 9: 2541 - 2552

[132] Sears MR. Trends in the prevalence of asthma. Chest 2014; 145: $219-225$

[133] Pearce N, Ait-Khaled N, Beasley R et al. Worldwide trends in the prevalence of asthma symptoms: phase III of the International Study of Asthma and Allergies in Childhood (ISAAC). Thorax 2007; 62: $758-766$

[134] Nowak D, Volmer T, Wettengel R. [Bronchial asthma-a cost of illness analysis]. Pneumologie 1996; 50: $364-371$

[135] Schramm B, Ehlken B, Smala A et al. Cost of illness of atopic asthma and seasonal allergic rhinitis in Germany: 1-yr retrospective study. Eur Respir ] 2003; 21: 116-122

[136] Bousquet J, Jeffery PK, Busse WW et al. Asthma. From bronchoconstriction to airways inflammation and remodeling. Am J Respir Crit Care Med 2000; 161: 1720-1745

[137] Kelly HW, Sternberg AL, Lescher R et al. Effect of inhaled glucocorticoids in childhood on adult height. N Engl J Med 2012; 367: 904 912

[138] Suissa S, Ernst P. Inhaled corticosteroids: impact on asthma morbidity and mortality. J Allergy Clin Immunol 2001; 107: 937-944

[139] Schultze-Werninghaus G. [Asthma mortality in Germany - a critical evaluation]. Pneumologie 2010; 64: 392

[140] Brand PL, Baraldi E, Bisgaard $\mathrm{H}$ et al. Definition, assessment and treatment of wheezing disorders in preschool children: an evidencebased approach. Eur Respir J 2008; 32: 1096 - 1110

[141] Brand PL, Caudri D, Eber E et al. Classification and pharmacologica treatment of preschool wheezing: changes since 2008. Eur Respir J 2014; 43: $1172-1177$

[142] Gotz M, Eber E, Frischer T et al. Rezidivierende obstruktive Bronchitis und Asthma bronchiale im Vorschulalter. Konsensuspapier der Österreichischen Gesellschaft für Kinder- und Jugendheilkunde (ÖGKJ) und der Österreichischen Gesellschaft für Pneumologie (ÖGP). Monatsschr Kinderheilkd 2006; 154: 679-684

[143] Quanjer PH, Stanojevic S, Cole TJ et al. Multi-ethnic reference values for spirometry for the 3-95-yr age range: the global lung function 2012 equations. Eur Respir J 2012; 40: 1324 - 1343

[144] Jenkins C, Seccombe L, Tomlins R. Investigating asthma symptoms in primary care. BMJ 2012; 344: e2734

[145] Cockcroft DW, Murdock KY, Berscheid BA et al. Sensitivity and specificity of histamine PC20 determination in a random selection of young college students. J Allergy Clin Immunol 1992; 89: 23 - 30

[146] Schneider A, Gindner L, Tilemann L et al. Diagnostic accuracy of spirometry in primary care. BMC Pulm Med 2009; 9: 31

[147] O'Byrne PM. Conclusion: Airway hyperresponsiveness in asthma: its measurement and clinical significance. Chest 2010; 138: 44S-45S

[148] Joos GF, O'Connor B, Anderson SD et al. Indirect airway challenges. Eur Respir J 2003; 21: 1050 - 1068

[149] Criée CP, Sorichter S, Smith H] et al. Body plethysmography - its principles and clinical use. Respir Med 2011; 105: 959-971

[150] Lex C, Zacharasiewicz A, Schulze J et al. Bronchiale Provokation im Kindes und Jugendalter. Konsensusstatement der AG Lungenfunktion der Gesellschaft Pädiatrische Pneumologie. Monatsschrift Kinderheilkd 2015; 163: 826-832

[151] Moeller A, Carlsen KH, Sly PD et al. Monitoring asthma in childhood: lung function, bronchial responsiveness and inflammation. Eur Respir Rev 2015; 24: $204-215$ 
[152] Brzozowska A, Majak P, Grzelewski T et al. Measurement of specific airway resistance decreased the risk of delay in asthma diagnosis in children. Allergy Asthma Proc 2009; 30: 47-54

[153] Magnussen H, Richter K, Taube C. Are chronic obstructive pulmonary disease (COPD) and asthma different diseases? Clin Exp Allergy 1998; 28: $187-194$

[154] Reddel HK, Salome CM, Peat JK et al. Which index of peak expiratory flow is most useful in the management of stable asthma? Am J Respir Crit Care Med 1995; 151: 1320-1325

[155] Petsky HL, Cates C], Lasserson TJ et al. A systematic review and metaanalysis: tailoring asthma treatment on eosinophilic markers (exhaled nitric oxide or sputum eosinophils). Thorax 2012; 67: 199-208

[156] Dweik RA, Boggs PB, Erzurum SC et al. An official ATS clinical practice guideline: interpretation of exhaled nitric oxide levels (FENO) for clinical applications. Am J Respir Crit Care Med 2011; 184: 602 - 615

[157] Pavord ID, Shaw DE, Gibson PG et al. Inflammometry to assess airway diseases. Lancet 2008; 372: 1017-1019

[158] Porsbjerg C, Baines K, Gibson P et al. IL-33 is related to innate immune activation and sensitization to HDM in mild steroid-free asthma. Clin Exp Allergy 2016; 46: $564-574$

[159] Donohue JF, Jain N. Exhaled nitric oxide to predict corticosteroid responsiveness and reduce asthma exacerbation rates. Respir Med 2013; 107: 943 - 952

[160] Pedrosa M, Cancelliere N, Barranco P et al. Usefulness of exhaled nitric oxide for diagnosing asthma. J Asthma 2010; 47: 817-821

[161] Elmasri M, Romero KM, Gilman RH et al. Longitudinal assessment of high versus low levels of fractional exhaled nitric oxide among children with asthma and atopy. Lung 2014; 192: 305-312

[162] Karrasch S, Linde K, Rücker $G$ et al. Accuracy of FENO for diagnosing asthma: a systematic review. Thorax 2017; 72: 109-116

[163] Petsky HL, Kew KM, Chang AB. Exhaled nitric oxide levels to guide treatment for children with asthma. Cochrane Database Syst Rev 2016; 11: CD011439

[164] Petsky HL, Kew KM, Turner C et al. Exhaled nitric oxide levels to guide treatment for adults with asthma. Cochrane Database Syst Rev 2016; 9: CD011440

[165] Lehtimäki L, Csonka P, Mäkinen E et al. Predictive value of exhaled nitric oxide in the management of asthma: a systematic review. Eur Respir J 2016; 48: 706 - 714

[166] Pijnenburg MW, Hofhuis W, Hop WC et al. Exhaled nitric oxide predicts asthma relapse in children with clinical asthma remission. Thorax 2005; 60: 215-218

[167] Zacharasiewicz A, Wilson N, Lex C et al. Clinical use of noninvasive measurements of airway inflammation in steroid reduction in children. Am J Respir Crit Care Med 2005; 171: 1077-1082

[168] McNicholl DM, Stevenson M, McGarvey LP et al. The utility of fractional exhaled nitric oxide suppression in the identification of nonadherence in difficult asthma. Am J Respir Crit Care Med 2012; 186: $1102-1108$

[169] Powell H, Murphy VE, Taylor DR et al. Management of asthma in pregnancy guided by measurement of fraction of exhaled nitric oxide: a double-blind, randomised controlled trial. Lancet 2011; 378: $983-990$

[170] Höffken G, Lorenz J, Kern W et al. Guidelines of the Paul-EhrlichSociety of Chemotherapy, the German Respiratory Diseases Society, the German Infectious Diseases Society and of the Competence Network CAPNETZ for the Management of Lower Respiratory Tract Infections and Community-acquired Pneumonia. Pneumologie 2010; 64: 149-154

[171] Graif Y, Yigla M, Tov N et al. Value of a negative aeroallergen skinprick test result in the diagnosis of asthma in young adults: correlative study with methacholine challenge testing. Chest 2002; 122: $821-825$
[172] Chan EY, Dundas I, Bridge PD et al. Skin-prick testing as a diagnostic aid for childhood asthma. Pediatr Pulmonol 2005; 39: 558- 562

[173] Melioli G, Passalacqua G, Canonica GW et al. Component-resolved diagnosis in pediatric allergic rhinoconjunctivitis and asthma. Curr Opin Allergy Clin Immunol 2013; 13: 446-451

[174] Ollert M, Mari A. In vitro allergy diagnosis - molecules and component-resolved diagnosis. In: Akdis CA, Hrsg. Global Atlas of Allergy. Zurich, Switzerland: European Academy of Allergy and Clinical Immunology; 2014: $168-170$

[175] Mehl A, Niggemann B, Keil T et al. Skin prick test and specific serum $\mathrm{lgE}$ in the diagnostic evaluation of suspected cow's milk and hen's egg allergy in children: does one replace the other? Clin Exp Allergy 2012; 42: $1266-1272$

[176] Ricci G, Capelli M, Miniero R et al. A comparison of different allergometric tests, skin prick test, Pharmacia UniCAP and ADVIA Centaur, for diagnosis of allergic diseases in children. Allergy 2003; 58: 38 45

[177] Gibson PG, McDonald VM, Marks GB. Asthma in older adults. Lancet 2010; 376: 803-813

[178] Price DB, Yawn BP, Jones RC. Improving the differential diagnosis of chronic obstructive pulmonary disease in primary care. Mayo Clin Proc 2010; 85: $1122-1129$

[179] Dima E, Rovina N, Gerassimou C et al. Pulmonary function tests, sputum induction, and bronchial provocation tests: diagnostic tools in the challenge of distinguishing asthma and COPD phenotypes in clinical practice. Int J Chron Obstruct Pulmon Dis 2010; 5: 287-296

[180] Silvestri IC, Pereira CA, Rodrigues SC. Comparison of spirometric changes in the response to bronchodilators of patients with asthma or chronic obstructive pulmonary disease. J Bras Pneumol 2008; 34 : $675-682$

[181] Barnes P]. Against the Dutch hypothesis: asthma and chronic obstructive pulmonary disease are distinct diseases. Am J Respir Crit Care Med 2006; 174: 240-243

[182] Barnes PJ. Asthma-COPD Overlap. Chest 2016; 149: $7-8$

[183] http://ginasthma.org/asthma-copd-and-asthma-copd-overlap-syndrome-acos/

[184] Barnes NC. Can guideline-defined asthma control be achieved? The Gaining Optimal Asthma Control study Am J Respir Crit Care Med 2004; 170: $830-831$

[185] Bateman ED, Buhl R, O'Byrne PM et al. Predictors of asthma control and a risk score for exacerbations. Am J Respir Crit Care Med 2012; 185: A5667

[186] Bateman ED, Reddel HK, Eriksson G et al. Overall asthma control: the relationship between current control and future risk. J Allergy Clin Immunol 2010; 125: 600-608

[187] van Essen-Zandvliet EE, Hughes MD, Waalkens H] et al. Effects of 22 months of treatment with inhaled corticosteroids and/or beta-2agonists on lung function, airway responsiveness, and symptoms in children with asthma. The Dutch Chronic Non-specific Lung Disease Study Group. Am Rev Respir Dis 1992; 146: 547 - 554

[188] Haahtela T, Järvinen M, Kava T et al. Comparison of a beta 2-agonist, terbutaline, with an inhaled corticosteroid, budesonide, in newly detected asthma. N Engl J Med 1991; 325: 388 - 392

[189] Kerrebijn KF, van Essen-Zandvliet EE, Neijens HJ. Effect of long-term treatment with inhaled corticosteroids and beta-agonists on the bronchial responsiveness in children with asthma. J Allergy Clin Immunol 1987; 79: 653-659

[190] Barnes PJ, Pedersen S. Efficacy and safety of inhaled corticosteroids in asthma. Report of a workshop held in Eze, France, October 1992. Am Rev Respir Dis 1993; 148: 1-26

[191] Djukanović R, Wilson JW, Britten KM et al. Effect of an inhaled corticosteroid on airway inflammation and symptoms in asthma. Am Rev Respir Dis 1992; 145: 669-674 
[192] Jeffery PK, Godfrey RW, Adelroth E et al. Effects of treatment on airway inflammation and thickening of basement membrane reticular collagen in asthma. A quantitative light and electron microscopic study. Am Rev Respir Dis 1992; 145: 890 - 899

[193] Gardiner PV, Ward C, Booth $\mathrm{H}$ et al. Effect of eight weeks of treatment with salmeterol on bronchoalveolar lavage inflammatory indices in asthmatics. Am J Respir Crit Care Med 1994; 150: 1006 - 1011

[194] Laitinen LA, Laitinen A, Haahtela T. A comparative study of the effects of an inhaled corticosteroid, budesonide, and a beta 2-agonist, terbutaline, on airway inflammation in newly diagnosed asthma: a randomized, double-blind, parallel-group controlled trial. J Allergy Clin Immunol 1992; 90: 32 - 42

[195] Anderson SD, Rozea PJ, Dolton R et al. Inhaled and oral bronchodilator therapy in exercise induced asthma. Aust N Z J Med 1975; 5: $544-550$

[196] Shaw R], Waller JF, Hetzel MR et al. Do oral and inhaled terbutaline have different effects on the lung? Br J Dis Chest 1982; 76: 171 - 176

[197] Powell H, Gibson PG. Initial starting dose of inhaled corticosteroids in adults with asthma: a systematic review. Thorax 2004; 59: 1041 1045

[198] Zhang L, Prietsch SO, Ducharme FM. Inhaled corticosteroids in children with persistent asthma: effects on growth. Cochrane Database Syst Rev 2014; 7: CD009471

[199] Godfrey S, Bar-Yishay E. Exercised-induced asthma revisited. Respir Med 1993; 87: $331-344$

[200] Parsons JP, Hallstrand TS, Mastronarde JG et al. An official American Thoracic Society clinical practice guideline: exercise-induced bronchoconstriction. Am J Respir Crit Care Med 2013; 187: 1016-1027

[201] Weiler JM, Brannan JD, Randolph CC et al. Exercise-induced bronchoconstriction update-2016. J Allergy Clin Immunol 2016; 138: $1292-1295$.e36

[202] [No authors listed]. Using beta 2-stimulants in asthma. Drug Ther Bull 1997; 35: 1-4

[203] O’Byrne PM, Barnes P], Rodriguez-Roisin R et al. Low dose inhaled budesonide and formoterol in mild persistent asthma: the OPTIMA randomized trial. Am J Respir Crit Care Med 2001; 164: 1392 - 1397

[204] Pauwels RA, Pedersen S, Busse WW et al. Early intervention with budesonide in mild persistent asthma: a randomised, double-blind trial. Lancet 2003; 361: 1071 - 1076

[205] Zeiger RS, Baker JW, Kaplan MS et al. Variability of symptoms in mild persistent asthma: baseline data from the MIAMI study. Respir Med 2004; 98: 898 - 905

[206] Reddel HK, Busse WW, Pedersen S et al. Should recommendations about starting inhaled corticosteroid treatment for mild asthma be based on symptom frequency: a post-hoc efficacy analysis of the START study. Lancet 2017; 389: 157-166

[207] Weinberger M, Hendeles L. Theophylline in asthma. N Engl J Med 1996; 334: 1380 - 1388

[208] Welsh E], Cates C]. Formoterol versus short-acting beta-agonists as relief medication for adults and children with asthma. Cochrane Database Syst Rev 2010; 9: CD008418

[209] Haahtela T, Järvinen M, Kava T et al. Effects of reducing or discontinuing inhaled budesonide in patients with mild asthma. N Engl J Med 1994; 331: $700-705$

[210] Lazarus SC, Boushey HA, Fahy JV et al. Long-acting beta2-agonist monotherapy vs continued therapy with inhaled corticosteroids in patients with persistent asthma: a randomized controlled trial. JAMA 2001; 285: $2583-2593$

[211] Lemanske RF, Sorkness CA, Mauger EA et al. Inhaled corticosteroid reduction and elimination in patients with persistent asthma receiving salmeterol: a randomized controlled trial. JAMA 2001; 285: $2594-2603$
[212] Simons FE. A comparison of beclomethasone, salmeterol, and placebo in children with asthma. Canadian Beclomethasone Dipropionate-Salmeterol Xinafoate Study Group. N Engl J Med 1997; 337: $1659-1665$

[213] Powell H, Gibson PG. Inhaled corticosteroid doses in asthma: an evidence-based approach. Med J Aust 2003; 178: $223-225$

[214] Wilson JW, Djukanović R, Howarth PH et al. Inhaled beclomethasone dipropionate downregulates airway lymphocyte activation in atopic asthma. Am J Respir Crit Care Med 1994; 149: 86 - 90

[215] The Childhood Asthma Management Program Research Group. Long-term effects of budesonide or nedocromil in children with asthma. N Engl ] Med 2000; 343: $1054-1063$

[216] Adams NP, Bestall JB, Malouf R et al. Inhaled beclomethasone versus placebo for chronic asthma. Cochrane Database Syst Rev 2005; 1 : CD002738

[217] Suissa S, Ernst P, Benayoun S et al. Low-dose inhaled corticosteroids and the prevention of death from asthma. N Engl J Med 2000; 343: $332-336$

[218] Bisgaard H. A metal aerosol holding chamber devised for young children with asthma. Eur Respir ] 1995; 8: 856 - 860

[219] Cates CC, Bara A, Crilly JA et al. Holding chambers versus nebulisers for beta-agonist treatment of acute asthma. Cochrane Database Syst Rev 2003; 3: CD000052

[220] Selroos O, Halme M. Effect of a volumatic spacer and mouth rinsing on systemic absorption of inhaled corticosteroids from a metered dose inhaler and dry powder inhaler. Thorax 1991; 46: 891-894

[221] Williamson IJ, Matusiewicz SP, Brown PH et al. Frequency of voice problems and cough in patients using pressurized aerosol inhaled steroid preparations. Eur Respir ] 1995; 8: 590-592

[222] Brown PH, Greening AP, Crompton GK. Large volume spacer devices and the influence of high dose beclomethasone dipropionate on hypothalamo-pituitary-adrenal axis function. Thorax 1993; 48: $233-238$

[223] Chauhan BF, Ducharme FM. Anti-leukotriene agents compared to inhaled corticosteroids in the management of recurrent and/or chronic asthma in adults and children. Cochrane Database Syst Rev 2012; 5: CD002314

[224] Lemanske RF, Mauger DT, Sorkness CA et al. Step-up therapy for children with uncontrolled asthma receiving inhaled corticosteroids. N Engl J Med 2010; 362: 975-985

[225] Philip G, Nayak AS, Berger WE et al. The effect of montelukast on rhinitis symptoms in patients with asthma and seasonal allergic rhinitis. Curr Med Res Opin 2004; 20: 1549-1558

[226] Wilson AM, Dempsey OJ, Sims E] et al. A comparison of topical budesonide and oral montelukast in seasonal allergic rhinitis and asthma. Clin Exp Allergy 2001; 31: 616-624

[227] Bjermer L, Bisgaard H, Bousquet J et al. Montelukast and fluticasone compared with salmeterol and fluticasone in protecting against asthma exacerbation in adults: one year, double blind, randomised, comparative trial. BMJ 2003; 327: 891 - 895

[228] Busse W, Nelson H, Wolfe J et al. Comparison of inhaled salmeterol and oral zafirlukast in patients with asthma. | Allergy Clin Immunol 1999; 103: 1075-1080

[229] Davies B, Brooks G, Devoy M. The efficacy and safety of salmeterol compared to theophylline: meta-analysis of nine controlled studies. Respir Med 1998; 92: 256-263

[230] Fish JE, Israel E, Murray JJ et al. Salmeterol powder provides significantly better benefit than montelukast in asthmatic patients receiving concomitant inhaled corticosteroid therapy. Chest 2001; 120 : $423-430$ 
[231] Nelson HS, Busse WW, Kerwin E et al. Fluticasone propionate/salmeterol combination provides more effective asthma control than low-dose inhaled corticosteroid plus montelukast. J Allergy Clin Immunol 2000; 106: 1088-1095

[232] Ringdal N, Eliraz A, Pruzinec R et al. The salmeterol/fluticasone combination is more effective than fluticasone plus oral montelukast in asthma. Respir Med 2003; 97: 234-241

[233] Shah L, Wilson AJ, Gibson PG et al. Long acting beta-agonists versus theophylline for maintenance treatment of asthma. Cochrane Database Syst Rev 2003; 3: CD001281

[234] Ni Chroinin M, Greenstone I, Lasserson TJ et al. Addition of inhaled long-acting beta2-agonists to inhaled steroids as first line therapy for persistent asthma in steroid-naive adults and children. Cochrane Database Syst Rev 2009; 4: CD005307

[235] Lazarinis N, Jørgensen L, Ekström T et al. Combination of budesonide/formoterol on demand improves asthma control by reducing exercise-induced bronchoconstriction. Thorax 2014; 69: 130-136

[236] American Lung Association Asthma Clinical Research Centers. Clinical trial of low-dose theophylline and montelukast in patients with poorly controlled asthma. Am J Respir Crit Care Med 2007; 175: $235-242$

[237] Dahl R, Larsen BB, Venge P. Effect of long-term treatment with inhaled budesonide or theophylline on lung function, airway reactivity and asthma symptoms. Respir Med 2002; 96: 432-438

[238] Evans D], Taylor DA, Zetterstrom O et al. A comparison of low-dose inhaled budesonide plus theophylline and high-dose inhaled budesonide for moderate asthma. N Engl J Med 1997; 337: 1412-1418

[239] Lim S, Jatakanon A, Gordon D et al. Comparison of high dose inhaled steroids, low dose inhaled steroids plus low dose theophylline, and low dose inhaled steroids alone in chronic asthma in general practice. Thorax 2000; 55: 837-841

[240] Reed CE, Offord KP, Nelson HS et al. Aerosol beclomethasone dipropionate spray compared with theophylline as primary treatment for chronic mild-to-moderate asthma. The American Academy of Allergy, Asthma and Immunology Beclomethasone Dipropionate-Theophylline Study Group. J Allergy Clin Immunol 1998; 101: 14-23

[241] Rivington RN, Boulet LP, Côté ] et al. Efficacy of Uniphyl, salbutamol, and their combination in asthmatic patients on high-dose inhaled steroids. Am J Respir Crit Care Med 1995; 151: 325-332

[242] Tinkelman DG, Reed CE, Nelson HS et al. Aerosol beclomethasone dipropionate compared with theophylline as primary treatment of chronic, mild to moderately severe asthma in children. Pediatrics 1993; 92: $64-77$

[243] Ukena D, Harnest U, Sakalauskas R et al. Comparison of addition of theophylline to inhaled steroid with doubling of the dose of inhaled steroid in asthma. Eur Respir J 1997; 10: 2754-2760

[244] Tsiu SJ, Self TH, Burns R. Theophylline toxicity: update. Ann Allergy 1990; 64: $241-257$

[245] Guevara JP, Ducharme FM, Keren R et al. Inhaled corticosteroids versus sodium cromoglycate in children and adults with asthma. Cochrane Database Syst Rev 2006; 2: CD003558

[246] Sridhar AV, McKean M. Nedocromil sodium for chronic asthma in children. Cochrane Database Syst Rev 2006; 3: CD004108

[247] Ducharme FM, Ni Chroinin M, Greenstone I et al. Addition of longacting beta2-agonists to inhaled corticosteroids versus same dose inhaled corticosteroids for chronic asthma in adults and children. Cochrane Database Syst Rev 2010; 5: CD005535

[248] Kesten S, Chapman KR, Broder I et al. A three-month comparison of twice daily inhaled formoterol versus four times daily inhaled albuterol in the management of stable asthma. Am Rev Respir Dis 1991; 144: $622-625$
[249] Pauwels RA, Löfdahl CG, Postma DS et al. Effect of inhaled formoterol and budesonide on exacerbations of asthma. Formoterol and Corticosteroids Establishing Therapy (FACET) International Study Group. N Engl J Med 1997; 337: 1405-1411

[250] Pearlman DS, Chervinsky P, LaForce C et al. A comparison of salmeterol with albuterol in the treatment of mild-to-moderate asthma. N Engl J Med 1992; 327: 1420-1425

[251] Shrewsbury S, Pyke S, Britton M. Meta-analysis of increased dose of inhaled steroid or addition of salmeterol in symptomatic asthma (MIASMA). BMJ 2000; 320: 1368-1373

[252] Wenzel SE, Lumry W, Manning M et al. Efficacy, safety, and effects on quality of life of salmeterol versus albuterol in patients with mild to moderate persistent asthma. Ann Allergy Asthma Immunol 1998; 80: $463-470$

[253] Bateman ED, Harrison TW, Quirce S et al. Overall asthma control achieved with budesonide/formoterol maintenance and reliever therapy for patients on different treatment steps. Respir Res 2011; 12: 38

[254] Cates C], Karner C. Combination formoterol and budesonide as maintenance and reliever therapy versus current best practice (including inhaled steroid maintenance), for chronic asthma in adults and children. Cochrane Database Syst Rev 2013; 4: CD007313

[255] Kew KM, Karner C, Mindus SM et al. Combination formoterol and budesonide as maintenance and reliever therapy versus combination inhaler maintenance for chronic asthma in adults and children. Cochrane Database Syst Rev 2013; 12: CD009019

[256] Papi A, Corradi M, Pigeon-Francisco C et al. Beclometasone-formoterol as maintenance and reliever treatment in patients with asthma: a double-blind, randomised controlled trial. Lancet Respir Med 2013; 1: 23-31

[257] Patel M, Pilcher ], Pritchard A et al. Efficacy and safety of maintenance and reliever combination budesonide-formoterol inhaler in patients with asthma at risk of severe exacerbations: a randomised controlled trial. Lancet Respir Med 2013; 1: $32-42$

[258] Aubier M, Pieters WR, Schlösser NJ et al. Salmeterol/fluticasone propionate (50/500 microg) in combination in a Diskus inhaler (Seretide) is effective and safe in the treatment of steroid-dependent asthma. Respir Med 1999; 93: 876-884

[259] Bateman E. Salmeterol/Fluticasone Propionate Combination. Drugs 1999; 57: 941 - 943

[260] Chapman KR, Ringdal N, Backer V et al. Salmeterol and fluticasone propionate (50/250 microg) administered via combination Diskus inhaler: as effective as when given via separate Diskus inhalers. Can Respir J 1999; 6: 45-51

[261] Nelson HS, Chapman KR, Pyke SD et al. Enhanced synergy between fluticasone propionate and salmeterol inhaled from a single inhaler versus separate inhalers. J Allergy Clin Immunol 2003; 112: 29-36

[262] Van den Berg NJ, Ossip MS, Hederos CA et al. Salmeterol/fluticasone propionate $(50 / 100$ microg) in combination in a Diskus inhaler (Seretide) is effective and safe in children with asthma. Pediatr Pulmonol 2000; 30: $97-105$

[263] Zetterström O, Buhl R, Mellem H et al. Improved asthma control with budesonide/formoterol in a single inhaler, compared with budesonide alone. Eur Respir J 2001; 18: 262 - 268

[264] Turner S, Richardson K, Murray C et al. Long-Acting $\beta$-Agonist in Combination or Separate Inhaler as Step-Up Therapy for Children with Uncontrolled Asthma Receiving Inhaled Corticosteroids. J Allergy Clin Immunol Pract 2017; 5: 99-106 .e3

[265] Ducharme FM, Ni Chroinin M, Greenstone I et al. Addition of longacting beta2-agonists to inhaled steroids versus higher dose inhaled steroids in adults and children with persistent asthma. Cochrane Database Syst Rev 2010; 4: CD005533 
[266] Szefler SJ, Martin RJ, King TS et al. Significant variability in response to inhaled corticosteroids for persistent asthma. J Allergy Clin Immunol 2002; 109: 410-418

[267] Greening AP, Ind PW, Northfield M et al. Added salmeterol versus higher-dose corticosteroid in asthma patients with symptoms on existing inhaled corticosteroid. Allen \& Hanburys Limited UK Study Group. Lancet 1994; 344: 219-224

[268] Woolcock A, Lundback B, Ringdal N et al. Comparison of addition of salmeterol to inhaled steroids with doubling of the dose of inhaled steroids. Am J Respir Crit Care Med 1996; 153: 1481 - 1488

[269] Busse WW, Chervinsky P, Condemi J et al. Budesonide delivered by Turbuhaler is effective in a dose-dependent fashion when used in the treatment of adult patients with chronic asthma. J Allergy Clin Immunol 1998; 101: 457-463

[270] Lipworth BJ. Systemic adverse effects of inhaled corticosteroid therapy: A systematic review and meta-analysis. Arch Intern Med 1999; 159: $941-955$

[271] Chauhan BF, Ducharme FM. Addition to inhaled corticosteroids of long-acting beta2-agonists versus anti-leukotrienes for chronic asthma. Cochrane Database Syst Rev 2014; 1: CD003137

[272] Laviolette M, Malmstrom K, Lu S et al. Montelukast added to inhaled beclomethasone in treatment of asthma. Montelukast/Beclomethasone Additivity Group. Am J Respir Crit Care Med 1999; 160: 1862 1868

[273] Price DB, Hernandez D, Magyar P et al. Randomised controlled trial of montelukast plus inhaled budesonide versus double dose inhaled budesonide in adult patients with asthma. Thorax 2003; 58: 211 216

[274] Vaquerizo M], Casan P, Castillo J et al. Effect of montelukast added to inhaled budesonide on control of mild to moderate asthma. Thorax 2003; 58: $204-210$

[275] Virchow JC, Prasse A, Naya I et al. Zafirlukast improves asthma control in patients receiving high-dose inhaled corticosteroids. Am J Respir Crit Care Med 2000; 162: 578-585

[276] Crompton GK, Ayres JG, Basran G et al. Comparison of oral bambuterol and inhaled salmeterol in patients with symptomatic asthma and using inhaled corticosteroids. Am J Respir Crit Care Med 1999; 159: $824-828$

[277] Persson G, Baas A, Knight A et al. One month treatment with the once daily oral beta 2-agonist bambuterol in asthmatic patients. Eur Respir J 1995; 8: $34-39$

[278] Wallaert B, Brun P, Ostinelli ] et al. A comparison of two long-acting beta-agonists, oral bambuterol and inhaled salmeterol, in the treatment of moderate to severe asthmatic patients with nocturnal symptoms. The French Bambuterol Study Group. Respir Med 1999; 93: $33-38$

[279] Murray CS, Thomas M, Richardson K et al. Comparative Effectiveness of Step-up Therapies in Children with Asthma Prescribed Inhaled Corticosteroids: A Historical Cohort Study. J Allergy Clin Immunol Pract 2017; 5: $1082-1090$

[280] O'Byrne PM, Naya IP, Kallen A et al. Increasing doses of inhaled corticosteroids compared to adding long-acting inhaled beta2-agonists in achieving asthma control. Chest 2008; 134: $1192-1199$

[281] Kerstjens HA, Disse B, Schröder-Babo W et al. Tiotropium improves lung function in patients with severe uncontrolled asthma: a randomized controlled trial. J Allergy Clin Immunol 2011; 128: 308 314

[282] Kerstjens HA, Engel M, Dahl R et al. Tiotropium in asthma poorly controlled with standard combination therapy. N Engl J Med 2012; 367: $1198-1207$

[283] Peters SP, Kunselman S], Icitovic N et al. Tiotropium bromide step-up therapy for adults with uncontrolled asthma. N Engl J Med 2010; 363: $1715-1726$
[284] Hamelmann E, Bateman ED, Vogelberg C et al. Tiotropium add-on therapy in adolescents with moderate asthma: A 1-year randomized controlled trial. J Allergy Clin Immunol 2016; 138: 441 - 450

[285] Vogelberg C, Moroni-Zentgraf P, Leonaviciute-Klimantaviciene M et al. A randomised dose-ranging study of tiotropium Respimat ${ }^{\circledR}$ in children with symptomatic asthma despite inhaled corticosteroids. Respir Res 2015; 16: 20

[286] Vogelberg C, Engel M, Moroni-Zentgraf P et al. Tiotropium in asthmatic adolescents symptomatic despite inhaled corticosteroids: a randomised dose-ranging study. Respir Med 2014; 108: 1268-1276

[287] Ni Chroinin M, Lasserson T], Greenstone I et al. Addition of longacting beta-agonists to inhaled corticosteroids for chronic asthma in children. Cochrane Database Syst Rev 2009; 3: CD007949

[288] Malo JL, Cartier A, Ghezzo H et al. Comparison of four-times-a-day and twice-a-day dosing regimens in subjects requiring 1200 micrograms or less of budesonide to control mild to moderate asthma. Respir Med 1995; 89: 537-543

[289] Toogood JH, Baskerville JC, Jennings B et al. Influence of dosing frequency and schedule on the response of chronic asthmatics to the aerosol steroid, budesonide. J Allergy Clin Immunol 1982; 70: 288 298

[290] Löfdahl CG, Reiss TF, Leff JA et al. Randomised, placebo controlled trial of effect of a leukotriene receptor antagonist, montelukast, on tapering inhaled corticosteroids in asthmatic patients. BMJ 1999; 319: $87-90$

[291] Tamaoki J, Kondo M, Sakai $\mathrm{N}$ et al. Leukotriene antagonist prevents exacerbation of asthma during reduction of high-dose inhaled corticosteroid. The Tokyo Joshi-Idai Asthma Research Group. Am J Respir Crit Care Med 1997; 155: 1235 - 1240

[292] Normansell R, Walker S, Milan S] et al. Omalizumab for asthma in adults and children. Cochrane Database Syst Rev 2014; 1: CD003559

[293] Garcia G, Magnan A, Chiron R et al. A proof-of-concept, randomized, controlled trial of omalizumab in patients with severe, difficult-tocontrol, nonatopic asthma. Chest 2013; 144: 411 - 419

[294] Bel EH, Wenzel SE, Thompson PJ et al. Oral glucocorticoid-sparing effect of mepolizumab in eosinophilic asthma. N Engl J Med 2014; 371: $1189-1197$

[295] Bjermer L, Lemiere C, Maspero J et al. Reslizumab for Inadequately Controlled Asthma With Elevated Blood Eosinophil Levels: A Randomized Phase 3 Study. Chest 2016; 150: 789-798

[296] Corren J, Weinstein S, Janka L et al. Phase 3 Study of Reslizumab in Patients With Poorly Controlled Asthma: Effects Across a Broad Range of Eosinophil Counts. Chest 2016; 150: 799-810

[297] Harrison BD, Stokes TC, Hart G] et al. Need for intravenous hydrocortisone in addition to oral prednisolone in patients admitted to hospital with severe asthma without ventilatory failure. Lancet 1986; $1: 181-184$

[298] O’Driscoll BR, Kalra S, Wilson M et al. Double-blind trial of steroid tapering in acute asthma. Lancet 1993; 341: 324-327

[299] Walsh LJ, Wong CA, Oborne J et al. Adverse effects of oral corticosteroids in relation to dose in patients with lung disease. Thorax 2001; 56: $279-284$

[300] Dunlap NE, Fulmer JD. Corticosteroid therapy in asthma. Clin Chest Med 1984; 5: 669-683

[301] Mash B, Bheekie A, Jones PW. Inhaled vs oral steroids for adults with chronic asthma. Cochrane Database Syst Rev 2001; 1: CD002160

[302] American College of Rheumatology Task Force on Osteoporosis Guidelines. Recommendations for the prevention and treatment of glucocorticoid-induced osteoporosis. Arthritis Rheum 1996; 39: $1791-1801$

[303] Eastell R, Reid DM, Compston J et al. A UK Consensus Group on management of glucocorticoid-induced osteoporosis: an update. J Intern Med 1998; 244: 271 -292 
[304] Vogelmeier C, Kardos P, Hofmann T et al. Nebulised budesonide using a novel device in patients with oral steroid-dependent asthma. Eur Respir J 2015; 45: 1273 - 1282

[305] Hill JM. Nebulised corticosteroids in the treatment of patients with asthma. Thorax 1999; 54: $661-663$

[306] Otulana BA, Varma N, Bullock A et al. High dose nebulized steroid in the treatment of chronic steroid-dependent asthma. Respir Med 1992; 86: 105 - 108

[307] Davies H, Olson L, Gibson P. Methotrexate as a steroid sparing agent for asthma in adults. Cochrane Database Syst Rev 2000; 2: CD000391

[308] Hill JM, Tattersfield AE. Corticosteroid sparing agents in asthma. Thorax 1995; 50: $577-582$

[309] Aaron SD, Dales RE, Pham B. Management of steroid-dependent asthma with methotrexate: a meta-analysis of randomized clinical trials. Respir Med 1998; 92: 1059-1065

[310] Marin MG. Low-dose methotrexate spares steroid usage in steroiddependent asthmatic patients: a meta-analysis. Chest 1997; 112: $29-33$

[311] Menz G, Buhl R, Gillissen A et al. Schwieriges Asthma: Klinische Phänotypen und Prinzipien der Therapie. Pneumologie 2002; 56: 132 137

[312] Pfaar O, Bachert C, Bufe A et al. Guideline on allergen-specific immunotherapy in IgE-mediated allergic diseases: S2k Guideline of the German Society for Allergology and Clinical Immunology (DGAKI), the Society for Pediatric Allergy and Environmental Medicine (GPA), the Medical Association of German Allergologists (AeDA), the Austrian Society for Allergy and Immunology (ÖGAI), the Swiss Society for Allergy and Immunology (SGAI), the German Society of Dermatology (DDG), the German Society of Oto- Rhino-Laryngology, Head and Neck Surgery (DGHNO-KHC), the German Society of Pediatrics and Adolescent Medicine (DGKJ), the Society for Pediatric Pneumology (GPP), the German Respiratory Society (DGP), the German Association of ENT Surgeons (BV-HNO), the Professional Federation of Paediatricians and Youth Doctors (BVKJ), the Federal Association of Pulmonologists (BDP) and the German Dermatologists Association (BVDD). Allergo J Int 2014; 23: 282 -319

[313] Mosbech H, Deckelmann R, de Blay F et al. Standardized quality (SQ) house dust mite sublingual immunotherapy tablet (ALK) reduces inhaled corticosteroid use while maintaining asthma control: a randomized, double-blind, placebo-controlled trial. J Allergy Clin Immunol 2014; 134: $568-575$

[314] Abramson MJ, Puy RM, Weiner JM. Injection allergen immunotherapy for asthma. Cochrane Database Syst Rev 2010; 8: CD001186

[315] Calderon MA, Casale TB, Nelson HS et al. An evidence-based analysis of house dust mite allergen immunotherapy: a call for more rigorous clinical studies. J Allergy Clin Immunol 2013; 132: 1322 - 1336

[316] Zielen S, Kardos P, Madonini E. Steroid-sparing effects with allergenspecific immunotherapy in children with asthma: a randomized controlled trial. J Allergy Clin Immunol 2010; 126: 942 - 949

[317] Jacobsen L, Niggemann B, Dreborg S et al. Specific immunotherapy has long-term preventive effect of seasonal and perennial asthma: 10-year follow-up on the PAT study. Allergy 2007; 62: $943-948$

[318] Bufe A, Eberle P, Franke-Beckmann E et al. Safety and efficacy in children of an SQ-standardized grass allergen tablet for sublingual immunotherapy. J Allergy Clin Immunol 2009; 123: 167-173

[319] Bousquet J, Scheinmann P, Guinnepain MT et al. Sublingual-swallow immunotherapy (SLIT) in patients with asthma due to house-dust mites: a double-blind, placebo-controlled study. Allergy 1999; 54 : $249-260$

[320] Pajno GB, Morabito L, Barberio G et al. Clinical and immunologic effects of long-term sublingual immunotherapy in asthmatic children sensitized to mites: a double-blind, placebo-controlled study. Allergy 2000; 55: $842-849$
[321] Valovirta E, Berstad AK, de Blic ] et al. Design and recruitment for the GAP trial, investigating the preventive effect on asthma development of an SQ-standardized grass allergy immunotherapy tablet in children with grass pollen-induced allergic rhinoconjunctivitis. Clin Ther 2011; 33: 1537 - 1546

[322] http://www.dgaki.de/leitlinien/s2k-leitlinie-sit/sit-produkte-studien-zulassung/

[323] http://www.atemwegsliga.de/

[324] Knipel V, Criée CP, Windisch W. [Correct inhalation therapy: instructions provided by Internet-based video screens. An initiative of the German Airway League]. Pneumologie 2013; 67: 157 - 161

[325] Dekhuijzen PN, Vincken W, Virchow JC et al. Prescription of inhalers in asthma and COPD: towards a rational, rapid and effective approach. Respir Med 2013; 107: 1817 - 1821

[326] Lavorini F. Inhaled drug delivery in the hands of the patient. J Aeroso Med Pulm Drug Deliv 2014; 27: 414-418

[327] Scichilone N. Asthma control: the right inhaler for the right patient. Adv Ther 2015; 32: 285-292

[328] Laube BL, Janssens HM, de Jongh FH et al. What the pulmonary specialist should know about the new inhalation therapies. Eur Respir J 2011; 37: 1308-1331

[329] Bosnic-Anticevich SZ, Sinha H, So S et al. Metered-dose inhaler technique: the effect of two educational interventions delivered in community pharmacy over time. J Asthma 2010; 47: 251 - 256

[330] van der Meer V, van Stel HF, Bakker MJ et al. Weekly self-monitoring and treatment adjustment benefit patients with partly controlled and uncontrolled asthma: an analysis of the SMASHING study. Respir Res 2010; 11: 74

[331] Bjermer L. The importance of continuity in inhaler device choice for asthma and chronic obstructive pulmonary disease. Respiration 2014; 88: $346-352$

[332] Thomas M, Price D, Chrystyn $\mathrm{H}$ et al. Inhaled corticosteroids for asthma: impact of practice level device switching on asthma control. BMC Pulm Med 2009; 9: 1

[333] Leach CL, Colice GL. A pilot study to assess lung deposition of HFAbeclomethasone and CFC-beclomethasone from a pressurized metered dose inhaler with and without add-on spacers and using varying breathhold times. J Aerosol Med Pulm Drug Deliv 2010; 23 : $355-361$

[334] Nair A, Menzies D, Hopkinson P et al. In vivo comparison of the relative systemic bioavailability of fluticasone propionate from three anti-static spacers and a metered dose inhaler. Br J Clin Pharmacol 2009; 67: 191 - 198

[335] Small M, Anderson P, Vickers A et al. Importance of inhaler-device satisfaction in asthma treatment: real-world observations of physician-observed compliance and clinical/patient-reported outcomes. Adv Ther 2011; 28: $202-212$

[336] Williams LK, Peterson EL, Wells K et al. A cluster-randomized trial to provide clinicians inhaled corticosteroid adherence information for their patients with asthma. J Allergy Clin Immunol 2010; 126: 225 231

[337] Mehuys E, Van Bortel L, De Bolle L et al. Effectiveness of pharmacist intervention for asthma control improvement. Eur Respir ] 2008; 31 $790-799$

[338] Papi A, Canonica GW, Maestrelli P et al. Rescue use of beclomethasone and albuterol in a single inhaler for mild asthma. $N$ Engl J Med 2007; 356: $2040-2052$

[339] Martin AR, Finlay WH. Nebulizers for drug delivery to the lungs. Expert Opin Drug Deliv 2015; 12: 889-900

[340] Hess DR. The mask for noninvasive ventilation: principles of design and effects on aerosol delivery. J Aerosol Med 2007; 20: S85-\$99 
[341] Dhand R. Aerosol delivery during mechanical ventilation: from basic techniques to new devices. J Aerosol Med Pulm Drug Deliv 2008; 21: $45-60$

[342] Ditcham W, Murdzoska J, Zhang G et al. Lung deposition of 99mTcradiolabeled albuterol delivered through a pressurized metered dose inhaler and spacer with facemask or mouthpiece in children with asthma. J Aerosol Med Pulm Drug Deliv 2014; 27: S63 - S75

[343] Gibson PG, Powell H. Written action plans for asthma: an evidencebased review of the key components. Thorax 2004; 59: 94 - 99

[344] Rodrigo G], Castro-Rodriguez JA. Anticholinergics in the treatment of children and adults with acute asthma: a systematic review with meta-analysis. Thorax 2005; 60: 740-746

[345] Cates C], Welsh EJ, Rowe BH. Holding chambers (spacers) versus nebulisers for beta-agonist treatment of acute asthma. Cochrane Database Syst Rev 2013; 9: CD000052

[346] Silverman RA, Osborn $\mathrm{H}$, Runge J et al. IV magnesium sulfate in the treatment of acute severe asthma: a multicenter randomized controlled trial. Chest 2002; 122: 489-497

[347] Browne G], Penna AS, Phung X et al. Randomised trial of intravenous salbutamol in early management of acute severe asthma in children. Lancet 1997; 349: $301-305$

[348] Papo MC, Frank ], Thompson AE. A prospective, randomized study of continuous versus intermittent nebulized albuterol for severe status asthmaticus in children. Crit Care Med 1993; 21: 1479-1486

[349] Craven D, Kercsmar CM, Myers TR et al. Ipratropium bromide plus nebulized albuterol for the treatment of hospitalized children with acute asthma. J Pediatr 2001; 138: 51 - 58

[350] Bhogal SK, McGillivray D, Bourbeau J et al. Early administration of systemic corticosteroids reduces hospital admission rates for children with moderate and severe asthma exacerbation. Ann Emerg Med 2012; 60: 84-91

[351] Ream RS, Loftis LL, Albers GM et al. Efficacy of IV theophylline in children with severe status asthmaticus. Chest 2001; 119: 1480 1488

[352] Wheeler DS, Jacobs BR, Kenreigh CA et al. Theophylline versus terbutaline in treating critically ill children with status asthmaticus: a prospective, randomized, controlled trial. Pediatr Crit Care Med 2005; 6: $142-147$

[353] Pabon H, Monem G, Kissoon N. Safety and efficacy of magnesium sulfate infusions in children with status asthmaticus. Pediatr Emerg Care 1994; 10: $200-203$

[354] Markovitz B. Does magnesium sulphate have a role in the management of paediatric status asthmaticus? Arch Dis Child 2002; 86: $381-382$

[355] Hartling L, Fernandes RM, Bialy L et al. Steroids and bronchodilators for acute bronchiolitis in the first two years of life: systematic review and meta-analysis. BMJ 2011; 342: d1714

[356] Andrews T, McGintee E, Mittal MK et al. High-dose continuous nebulized levalbuterol for pediatric status asthmaticus: a randomized trial. J Pediatr 2009; 155: $205-210$

[357] Craig VL, Bigos D, Brilli RJ. Efficacy and safety of continuous albuterol nebulization in children with severe status asthmaticus. Pediatr Emerg Care 1996; 12: 1 - 5

[358] Stephanopoulos DE, Monge R, Schell KH et al. Continuous intravenous terbutaline for pediatric status asthmaticus. Crit Care Med 1998; $26: 1744-1748$

[359] Kim IK, Phrampus E, Venkataraman S et al. Helium/oxygen-driven albuterol nebulization in the treatment of children with moderate to severe asthma exacerbations: a randomized, controlled trial. Pediatrics 2005; 116: $1127-1133$

[360] Nievas IF, Anand K]. Severe acute asthma exacerbation in children: a stepwise approach for escalating therapy in a pediatric intensive care unit. J Pediatr Pharmacol Ther 2013; 18: 88-104
[361] Brenner B, Corbridge T, Kazzi A. Intubation and mechanical ventilation of the asthmatic patient in respiratory failure. Proc Am Thorac Soc 2009; 6: $371-379$

[362] Moore WC, Evans MD, Bleecker ER et al. Safety of investigative bronchoscopy in the Severe Asthma Research Program. J Allergy Clin Immunol 2011; 128: 328 - 336

[363] Good JT, Kolakowski CA, Groshong SD et al. Refractory asthma: importance of bronchoscopy to identify phenotypes and direct therapy. Chest 2012; 141: 599-606

[364] Scala R. Noninvasive ventilation in severe acute asthma? Still far from the truth. Respir Care 2010; 55: 630-637

[365] Soroksky A, Klinowski E, Ilgyev E et al. Noninvasive positive pressure ventilation in acute asthmatic attack. Eur Respir Rev 2010; 19: 39 45

[366] Westhoff M, Schönhofer B, Neumann P et al. [Noninvasive Mechanical Ventilation in Acute Respiratory Failure]. Pneumologie 2015; 69: $719-756$

[367] Nowak R, Corbridge T, Brenner B. Noninvasive ventilation. Proc Am Thorac Soc 2009; 6: 367-370

[368] Schönhofer B, Kuhlen R, Neumann P et al. [Non-invasive mechanical ventilation in acute respiratory failure]. Pneumologie 2008; 62: 449-479

[369] Basnet S, Mander G, Andoh J et al. Safety, efficacy, and tolerability of early initiation of noninvasive positive pressure ventilation in pediatric patients admitted with status asthmaticus: a pilot study. Pediatr Crit Care Med 2012; 13: 393-398

[370] Carroll CL, Schramm CM. Noninvasive positive pressure ventilation for the treatment of status asthmaticus in children. Ann Allergy Asthma Immunol 2006; 96: 454-459

[371] Mayordomo-Colunga J, Medina A, Rey C et al. Non-invasive ventilation in pediatric status asthmaticus: a prospective observational study. Pediatr Pulmonol 2011; 46: 949-955

[372] Akingbola OA, Simakajornboon N, Hadley EFJr et al. Noninvasive positive-pressure ventilation in pediatric status asthmaticus. Pediatr Crit Care Med 2002; 3: $181-184$

[373] Venkataraman ST. Mechanical ventilation and respiratory care. In: Fuhrman BP, Zimmerman J], (Eds). Pediatric critical care. Philadelphia: Mosby; 2006

[374] Sodhi C, Singh S, Dandona PK. A study of the effect of yoga training on pulmonary functions in patients with bronchial asthma. Indian J Physiol Pharmacol 2009; 53: 169-174

[375] Holloway E, Ram FS. Breathing exercises for asthma. Cochrane Database Syst Rev 2004; 1: CD001277

[376] Holloway EA, West RJ. Integrated breathing and relaxation training (the Papworth method) for adults with asthma in primary care: a randomised controlled trial. Thorax 2007; 62: 1039-1042

[377] Shaw BS, Shaw I. Pulmonary function and abdominal and thoracic kinematic changes following aerobic and inspiratory resistive diaphragmatic breathing training in asthmatics. Lung 2011; 189: 131 139

[378] Freitas DA, Holloway EA, Bruno SS et al. Breathing exercises for adults with asthma. Cochrane Database Syst Rev 2013; 10 : CD001277

[379] Ritz T, Rosenfield D, Steele AM et al. Controlling asthma by training of Capnometry-Assisted Hypoventilation (CATCH) vs slow breathing: a randomized controlled trial. Chest 2014; 146: 1237-1247

[380] Barker NJ, Jones M, O'Connell NE et al. Breathing exercises for dysfunctional breathing/hyperventilation syndrome in children. Cochrane Database Syst Rev 2013; 12: CD010376

[381] Lehrer PM, Vaschillo E, Vaschillo B et al. Biofeedback treatment for asthma. Chest 2004; 126: $352-361$ 
[382] Thomas M, McKinley RK, Mellor S et al. Breathing exercises for asthma: a randomised controlled trial. Thorax 2009; 64: 55-61

[383] Chiang LC, Ma WF, Huang JL et al. Effect of relaxation-breathing training on anxiety and asthma signs/symptoms of children with moderate-to-severe asthma: a randomized controlled trial. Int J Nurs Stud 2009; 46: 1061 - 1070

[384] Blackhall K, Appleton S, Cates C]. Ionisers for chronic asthma. Cochrane Database Syst Rev 2003; 3: CD002986

[385] Warner JA, Marchant JL, Warner JO. Double blind trial of ionisers in children with asthma sensitive to the house dust mite. Thorax 1993; 48: $330-333$

[386] Nogrady SG, Furnass SB. Ionisers in the management of bronchial asthma. Thorax 1983; 38: $919-922$

[387] Yang ZY, Zhong HB, Mao C et al. Yoga for asthma. Cochrane Database Syst Rev 2016; 4: CD010346

[388] Huntley A, White AR, Ernst E. Relaxation therapies for asthma: a systematic review. Thorax 2002; 57: 127-131

[389] Yorke J, Fleming S, Shuldham C et al. Nonpharmacological interventions aimed at modifying health and behavioural outcomes for adults with asthma: a critical review. Clin Exp Allergy 2015; 45: $1750-1764$

[390] Ritz T, Meuret AE, Trueba AF et al. Psychosocial factors and behavioral medicine interventions in asthma. J Consult Clin Psychol 2013; 81: $231-250$

[391] Boyle RJ, Pedroletti C, Wickman M et al. Nocturnal temperature controlled laminar airflow for treating atopic asthma: a randomised controlled trial. Thorax 2012; 67: 215-221

[392] Pedroletti C, Millinger E, Dahlén B et al. Clinical effects of purified air administered to the breathing zone in allergic asthma: A doubleblind randomized cross-over trial. Respir Med 2009; 103: 1313 1319

[393] British Thoracic Society; Scottish Intercollegiate Guidelines Network. British Guideline on the Management of Asthma. A national clinical guideline. Verfügbar unter http://www.brit-thoracic.org.uk 2016

[394] Lingner H, Ernst S, Großhennig A et al. Asthma control and healthrelated quality of life one year after inpatient pulmonary rehabilitation: the ProKAR Study. J Asthma 2015; 52: 614-621

[395] Farin E, Opitz U, Jäckel WH et al. Pneumologische Rehabilitation: Langzeitdaten zur Lebensqualität und Prädiktoren der Behandlungseffekte. Phys Med Rehab Kuror 2009; 19: 75-84

[396] Schmidt J, Kaiser U, Kriz D et al. Die Davoser Outcome-Studie (DOS) - Ergebnisse stationärer pneumologischer und dermatologischer Heilbehandlungen im Spiegel wiederholter Nacherhebungen. Prävention und Rehabilitation 2014; 26: $119-140$

[397] Schultz K, Seidl H, Jelusic D et al. Effectiveness of pulmonary rehabilitation for patients with asthma: study protocol of a randomized controlled trial (EPRA). BMC Pulm Med 2017; 17: 49

[398] de Vries U. Asthma - Patientenschulung im Rahmen ambulanter und stationärer Rehabilitation. Regensburg: Roderer Verlag; 2004

[399] Nici L, Donner C, Wouters E et al. American Thoracic Society/European Respiratory Society statement on pulmonary rehabilitation. Am J Respir Crit Care Med 2006; 173: 1390-1413

[400] British Thoracic Society Standards of Care Subcommittee on Pulmonary Rehabilitation. Pulmonary rehabilitation. Thorax 2001; 56: $827-834$

[401] Ries AL, Bauldoff GS, Carlin BW et al. Pulmonary Rehabilitation: Joint ACCP/AACVPR Evidence-Based Clinical Practice Guidelines. Chest 2007; 131: 4S-42S

[402] Bergmann KC, Fischer], Schmitz M et al. [Inpatient pneumologic rehabilitation of adults: goals-diagnostic and therapeutic standardsresearch needs]. Pneumologie 1997; 51: 523-532
[403] World Health Organisation. (Hrsg). International Classification of Functioning, Disability and Health (ICF). 2001

[404] Fischer ], Raschke F. [Goal-oriented measurement of short-, medium- and long-term effects in pneumological rehabilitation]. Pneumologie 2000; 54: $458-463$

[405] Worth H, Petro W. [Recommendations for structure and contents of train-the-trainer seminars for educating patients with chronic obstructive respiratory tract diseases]. Pneumologie 1998; 52: $473-474$

[406] Spindler T, Rank M, Otto J. Veränderte psychosoziale Rahmenbedingungen erfordern Umdenken in der Rehabilitation. Kinder- und Jugendmedizin 2001; 5: $35-38$

[407] Szczepanski R, Jaeschke R, Spindler T et al. Preschoolers' and parents' asthma education trial (P2AET)-a randomized controlled study. Eur J Pediatr 2010; 169: 1051-1060

[408] Spindler T, Lob-Corzilius T. Qualitätmanagement in der Asthmaschulung von Kindern und Jugendlichen. 4. Auflage Wangen im Allgäu: iKuh-Verlag; 2013

[409] Bauer CP, Petermann F, Kiosz D et al. [Long-term effect of indoor rehabilitation on children and young people with moderate and severe asthma]. Pneumologie 2002; 56: 478-485

[410] Emtner M, Finne M, Stålenheim G. A 3-year follow-up of asthmatic patients participating in a 10-week rehabilitation program with emphasis on physical training. Arch Phys Med Rehabil 1998; 79: 539 544

[411] Petermann F, Gulyas A, Niebank K et al. Rehabilitationserfolg bei Kindern und Jugendlichen mit Asthma und Neurodermitis. Allergologie 2000; 23: 492 - 502

[412] Warschburger P, Busch S, Bauer CP et al. Health-related quality of life in children and adolescents with asthma: results from the ESTAR Study. J Asthma 2004; 41: $463-470$

[413] Cambach W, Wagenaar RC, Koelman TW et al. The long-term effects of pulmonary rehabilitation in patients with asthma and chronic obstructive pulmonary disease: a research synthesis. Arch Phys Med Rehabil 1999; 80: 103-111

[414] Cambach W, Chadwick-Straver RV, Wagenaar RC et al. The effects of a community-based pulmonary rehabilitation programme on exercise tolerance and quality of life: a randomized controlled trial. Eur Respir J 1997; 10: 104-113

[415] Foglio K, Bianchi L, Bruletti G et al. Long-term effectiveness of pulmonary rehabilitation in patients with chronic airway obstruction. Eur Respir J 1999; 13: 125-132

[416] www.asthmaschulung.de

[417] Gibson PG, Powell H, Coughlan J et al. Self-management education and regular practitioner review for adults with asthma. Cochrane Database Syst Rev 2003; 1: CD001117

[418] Gibson PG, Powell H, Coughlan J et al. Limited (information only) patient education programs for adults with asthma. Cochrane Database Syst Rev 2002; 2: CD001005

[419] Schacher C, Dhein Y, Münks-Lederer C et al. [Evaluation of a structured outpatient education program for adult asthmatics]. Dtsch Med Wochenschr 2006; 131: 606-610

[420] Powell H, Gibson PG. Options for self-management education for adults with asthma. Cochrane Database Syst Rev 2003; 1: CD004107

[421] Petro W, Wettengel R, Worth H. Empfehlungen zum strukturierten Patiententraining bei obstruktiven Atemwegserkrankungen. Med Klin 1995; 90: $515-519$

[422] Carson KV, Chandratilleke MG, Picot J et al. Physical training for asthma. Cochrane Database Syst Rev 2013; 9: CD001116

[423] Emtner M, Herala M, Stålenheim G. High-intensity physical training in adults with asthma. A 10-week rehabilitation program. Chest 1996; 109: $323-330$ 
[424] Mendes FA, Gonçalves RC, Nunes MP et al. Effects of aerobic training on psychosocial morbidity and symptoms in patients with asthma: a randomized clinical trial. Chest 2010; 138: 331 - 337

[425] Turner S, Eastwood P, Cook A et al. Improvements in symptoms and quality of life following exercise training in older adults with moderate/severe persistent asthma. Respiration 2011; 81: 302-310

[426] Dogra S, Kuk JL, Baker J et al. Exercise is associated with improved asthma control in adults. Eur Respir J 2011; 37: 318-323

[427] Beggs S, Foong YC, Le HC et al. Swimming training for asthma in children and adolescents aged 18 years and under. Cochrane Database Syst Rev 2013; 4: CD009607

[428] Worth H, Meyer A, Folgering $\mathrm{H}$ et al. [Recommendations of the German Respiratory League on sports and physical training for patients with obstructive respiratory tract diseases]. Pneumologie 2000; 54: $61-67$

[429] Siroux V, Pin I, Oryszczyn MP et al. Relationships of active smoking to asthma and asthma severity in the EGEA study. Epidemiological study on the Genetics and Environment of Asthma. Eur Respir J 2000; 15: $470-477$

[430] Ehrlich RI, Du Toit D, Jordaan E et al. Risk factors for childhood asthma and wheezing. Importance of maternal and household smoking. Am J Respir Crit Care Med 1996; 154: 681-688

[431] Chaudhuri R, Livingston E, McMahon AD et al. Effects of smoking cessation on lung function and airway inflammation in smokers with asthma. Am J Respir Crit Care Med 2006; 174: 127-133

[432] Rayens MK, Burkhart PV, Zhang M et al. Reduction in asthma-related emergency department visits after implementation of a smoke-free law. J Allergy Clin Immunol 2008; 122: 537 - 541 .e3

[433] Andreas S, Batra A, Behr ] et al. [Guidelines for smoking cessation in patients with COPD issued by the Deutsche Gesellschaft für Pneumologie und Beatmungsmedizin]. Pneumologie 2008; 62: 255-272

[434] Boulet LP, Franssen E. Influence of obesity on response to fluticasone with or without salmeterol in moderate asthma. Respir Med 2007; 101: $2240-2247$

[435] Lavoie KL, Bacon SL, Labrecque M et al. Higher BMI is associated with worse asthma control and quality of life but not asthma severity. Respir Med 2006; 100: 648-657

[436] Saint-Pierre P, Bourdin A, Chanez P et al. Are overweight asthmatics more difficult to control? Allergy 2006; 61: 79-84

[437] Sutherland ER, Goleva E, Strand M et al. Body mass and glucocorticoid response in asthma. Am J Respir Crit Care Med 2008; 178: 682 687

[438] Adeniyi FB, Young T. Weight loss interventions for chronic asthma. Cochrane Database Syst Rev 2012; 7: CD009339

[439] Moreira A, Bonini M, Garcia-Larsen V et al. Weight loss interventions in asthma: EAACl evidence-based clinical practice guideline (part I). Allergy 2013; 68: 425-439

[440] Boulet LP, Turcotte H, Martin J et al. Effect of bariatric surgery on airway response and lung function in obese subjects with asthma. Respir Med 2012; 106: 651-660

[441] Dixon AE, Pratley RE, Forgione PM et al. Effects of obesity and bariatric surgery on airway hyperresponsiveness, asthma control, and inflammation. J Allergy Clin Immunol 2011; 128: 508-515

[442] Scott HA, Gibson PG, Garg ML et al. Dietary restriction and exercise improve airway inflammation and clinical outcomes in overweight and obese asthma: a randomized trial. Clin Exp Allergy 2013; 43: $36-49$

[443] Castro M, Rubin AS, Laviolette M et al. Effectiveness and safety of bronchial thermoplasty in the treatment of severe asthma: a multicenter, randomized, double-blind, sham-controlled clinical trial. Am | Respir Crit Care Med 2010; 181: 116-124
[444] Thomson NC, Rubin AS, Niven RM et al. Long-term (5 year) safety of bronchial thermoplasty: Asthma Intervention Research (AIR) trial. BMC Pulm Med 2011; 11: 8

[445] Cox G, Thomson NC, Rubin AS et al. Asthma control during the year after bronchial thermoplasty. N Engl J Med 2007; 356: 1327-1337

[446] Wechsler ME, Laviolette M, Rubin AS et al. Bronchial thermoplasty: Long-term safety and effectiveness in patients with severe persistent asthma. J Allergy Clin Immunol 2013; 132: 1295-1302

[447] Cazzola M, Calzetta L, Bettoncelli G et al. Asthma and comorbid medical illness. Eur Respir J 2011; 38: 42 - 49

[448] Gershon AS, Wang C, Guan J et al. Burden of comorbidity in individuals with asthma. Thorax 2010; 65: 612-618

[449] Havemann BD, Henderson CA, El-Serag HB. The association between gastro-oesophageal reflux disease and asthma: a systematic review. Gut 2007; 56: $1654-1664$

[450] Maher MM, Darwish AA. Study of respiratory disorders in endoscopically negative and positive gastroesophageal reflux disease. Saudi J Gastroenterol 2010; 16: 84-89

[451] Prosser R, Carleton B, Smith A. The comorbidity burden of the treated asthma patient population in British Columbia. Chronic Dis Can 2010; 30: 46-55

[452] Tsai MC, Lin HL, Lin CC et al. Increased risk of concurrent asthma among patients with gastroesophageal reflux disease: a nationwide population-based study. Eur J Gastroenterol Hepatol 2010; 22 : $1169-1173$

[453] Khorasani EN, Fallahi GH, Mansouri F et al. The effect of omeprazole on asthmatic adolescents with gastroesophageal reflux disease. Allergy Asthma Proc 2008; 29: 517-520

[454] Sharma B, Sharma M, Daga MK et al. Effect of omeprazole and domperidone on adult asthmatics with gastroesophageal reflux. World ] Gastroenterol 2007; 13: $1706-1710$

[455] Shimizu Y, Dobashi K, Zhao J] et al. Proton pump inhibitor improves breath marker in moderate asthma with gastroesophageal reflux disease. Respiration 2007; 74: 558 - 564

[456] Chan WW, Chiou E, Obstein KL et al. The efficacy of proton pump inhibitors for the treatment of asthma in adults: a meta-analysis. Arch Intern Med 2011; 171: 620-629

[457] Writing Committee for the American Lung Association Asthma Clinical Research Centers; Holbrook JT. Wise RA. Gold BD et al. Lansoprazole for children with poorly controlled asthma: a randomized controlled trial. JAMA 2012; 307: 373-381

[458] Kiljander TO, Junghard O, Beckman O et al. Effect of esomeprazole $40 \mathrm{mg}$ once or twice daily on asthma: a randomized, placebo-controlled study. Am J Respir Crit Care Med 2010; 181: 1042 - 1048

[459] American Lung Association Asthma Clinical Research Centers; Mastronarde JG. Anthonisen NR. Castro M et al. Efficacy of esomeprazole for treatment of poorly controlled asthma. N Engl J Med 2009; 360: $1487-1499$

[460] Riscili BP, Parsons JP, Mastronarde JG. Treating silent reflux disease does not improve poorly controlled asthma. Cleve Clin J Med 2010 77: $155-160$

[461] Julien JY, Martin JG, Ernst P et al. Prevalence of obstructive sleep apnea-hypopnea in severe versus moderate asthma. J Allergy Clin Immunol 2009; 124: 371-376

[462] Teodorescu M, Consens FB, Bria WF et al. Predictors of habitual snoring and obstructive sleep apnea risk in patients with asthma. Chest 2009; 135: 1125 - 1132

[463] Teodorescu M, Polomis DA, Hall SV et al. Association of obstructive sleep apnea risk with asthma control in adults. Chest 2010; 138: $543-550$

[464] Yigla M, Tov N, Solomonov A et al. Difficult-to-control asthma and obstructive sleep apnea. J Asthma 2003; 40: 865 - 871 
[465] Deutsche Gesellschaft für Schlafforschung und Schlafmedizin. S3Leitlinie Nicht erholsamer Schlaf/Schlafstörungen - Kapitel „Schlafbezogene Atmungsstörungen“. Somnologie 2017; 20: S97-S180

[466] Ehlayel MS, Bener A, Sabbah A. Is high prevalence of vitamin D deficiency evidence for asthma and allergy risks? Eur Ann Allergy Clin Immunol 2011; 43: 81-88

[467] Li F, Peng M, Jiang L et al. Vitamin D deficiency is associated with decreased lung function in Chinese adults with asthma. Respiration 2011; 81: 469-475

[468] Erkkola M, Kaila M, Nwaru Bl et al. Maternal vitamin D intake during pregnancy is inversely associated with asthma and allergic rhinitis in 5-year-old children. Clin Exp Allergy 2009; 39: 875-882

[469] Todd DC, Armstrong S, D'Silva L et al. Effect of obesity on airway inflammation: a cross-sectional analysis of body mass index and sputum cell counts. Clin Exp Allergy 2007; 37: 1049-1054

[470] Kim KM, Kim SS, Kwon JW et al. Association between subcutaneous abdominal fat and airway hyperresponsiveness. Allergy Asthma Proc 2011; 32: 68-73

[471] Yoo S, Kim HB, Lee SY et al. Association between obesity and the prevalence of allergic diseases, atopy, and bronchial hyperresponsiveness in Korean adolescents. Int Arch Allergy Immunol 2011; 154: $42-48$

[472] Ciprandi G, Pistorio A, Tosca M et al. Body mass index, respiratory function and bronchial hyperreactivity in allergic rhinitis and asthma. Respir Med 2009; 103: 289-295

[473] Kilic H, Oguzulgen IK, Bakir F et al. Asthma in obese women: outcomes and factors involved. J Investig Allergol Clin Immunol 2011; 21: $290-296$

[474] Farah CS, Kermode JA, Downie SR et al. Obesity is a determinant of asthma control independent of inflammation and lung mechanics. Chest 2011; 140: 659-666

[475] McGinley B, Punjabi NM. Obesity, metabolic abnormalities, and asthma: establishing causal links. Am J Respir Crit Care Med 2011; 183: $424-425$

[476] Peters JI, McKinney JM, Smith B et al. Impact of obesity in asthma: evidence from a large prospective disease management study. Ann Allergy Asthma Immunol 2011; 106: 30-35

[477] Raviv S, Dixon AE, Kalhan R et al. Effect of obesity on asthma phenotype is dependent upon asthma severity. J Asthma 2011; 48: 98 104

[478] Thomsen SF, Duffy DL, Kyvik KO et al. Risk of asthma in adult twins with type 2 diabetes and increased body mass index. Allergy 2011; 66: $562-568$

[479] Minas M, Papaioannou Al, Tsaroucha A et al. Body composition in severe refractory asthma: comparison with COPD patients and healthy smokers. PloS one 2010; 5: e13233

[480] Song Y, Klevak A, Manson JE et al. Asthma, chronic obstructive pulmonary disease, and type 2 diabetes in the Women's Health Study. Diabetes Res Clin Pract 2010; 90: 365-371

[481] Dixon AE, Holguin F, Sood A et al. An official American Thoracic Society Workshop report: obesity and asthma. Proc Am Thorac Soc 2010; 7: 325-335

[482] Sinniah D. Obesity: links to diabetes and asthma. Intern Med J 2010; 40: $471-472$

[483] Grammer LC, Weiss KB, Pedicano JB et al. Obesity and asthma morbidity in a community-based adult cohort in a large urban area: the Chicago Initiative to Raise Asthma Health Equity (CHIRAH). J Asthma 2010; 47: $491-495$

[484] Ma J, Xiao L, Knowles SB. Obesity, insulin resistance and the prevalence of atopy and asthma in US adults. Allergy 2010; 65: $1455-$ 1463
[485] Camargo CA, Sutherland ER, Bailey W et al. Effect of increased body mass index on asthma risk, impairment and response to asthma controller therapy in African Americans. Curr Med Res Opin 2010; 26: $1629-1635$

[486] Wilson DH, Appleton SL, Taylor AW et al. Depression and obesity in adults with asthma: multiple comorbidities and management issues. Med J Aust 2010; 192: 381-383

[487] Farah CS, Boulet LP, Reddel HK. Obesity and asthma control in an urban population. J Allergy Clin Immunol 2010; 125: 769-770

[488] Jordan JG, Mann JR. Obesity and mortality in persons with obstructive lung disease using data from the NHANES III. South Med J 2010; 103: 323-330

[489] Uddenfeldt M, Janson C, Lampa E et al. High BMI is related to higher incidence of asthma, while a fish and fruit diet is related to a lowerResults from a long-term follow-up study of three age groups in Sweden. Respir Med 2010; 104: 972 - 980

[490] Pakhale S, Doucette S, Vandemheen K et al. A comparison of obese and nonobese people with asthma: exploring an asthma-obesity interaction. Chest 2010; 137: 1316-1323

[491] Hjellvik V, Tverdal A, Furu K. Body mass index as predictor for asthma: a cohort study of 118,723 males and females. Eur Respir J 2010; 35: $1235-1242$

[492] McHugh MK, Symanski E, Pompeii LA et al. Prevalence of asthma among adult females and males in the United States: results from the National Health and Nutrition Examination Survey (NHANES), 2001-2004. J Asthma 2009; 46: 759-766

[493] Ahmad N, Biswas S, Bae S et al. Association between obesity and asthma in US children and adolescents. J Asthma 2009; 46: 642 - 646

[494] Clerisme-Beaty E, Rand CS. The effect of obesity on asthma incidence: moving past the epidemiologic evidence. J Allergy Clin Immunol 2009; 123: 96 - 97

[495] Coogan PF, Palmer JR, O'Connor GT et al. Body mass index and asthma incidence in the Black Women's Health Study. J Allergy Clin Immunol 2009; 123: 89-95

[496] Mosen DM, Schatz M, Magid DJ et al. The relationship between obesity and asthma severity and control in adults. J Allergy Clin Immunol 2008; 122: $507-511$

[497] Eneli IU, Skybo T, Camargo CA. Weight loss and asthma: a systematic review. Thorax 2008; 63: 671-676

[498] McClean KM, Kee F, Young IS et al. Obesity and the lung: 1. Epidemiology. Thorax 2008; 63: 649-654

[499] Taylor B, Mannino D, Brown C et al. Body mass index and asthma severity in the National Asthma Survey. Thorax 2008; 63: 14-20

[500] Thomsen SF, Ulrik CS, Kyvik KO et al. Association between obesity and asthma in a twin cohort. Allergy 2007; 62: 1199-1204

[501] Sood A. Sex differences: implications for the obesity-asthma association. Exerc Sport Sci Rev 2011; 39: 48-56

[502] Forno E, Lescher R, Strunk R et al. Decreased response to inhaled steroids in overweight and obese asthmatic children. J Allergy Clin Immunol 2011; 127: 741 - 749

[503] Barranco P, Delgado J, Sastre J et al. Obesity is not associated with mild asthma diagnosis in a population of Spanish adults. J Asthma 2009; 46: $867-871$

[504] Musaad SM, Patterson T, Ericksen M et al. Comparison of anthropometric measures of obesity in childhood allergic asthma: central obesity is most relevant. J Allergy Clin Immunol 2009; 123: 1321 1327

[505] Thuesen BH, Husemoen LL, Hersoug LG et al. Insulin resistance as a predictor of incident asthma-like symptoms in adults. Clin Exp Allergy 2009; 39: 700-707 
[506] Lombardi C, Gargioni S, Gardinazzi A et al. Impact of bariatric surgery on pulmonary function and nitric oxide in asthmatic and nonasthmatic obese patients. J Asthma 2011; 48: 553-557

[507] Brancatisano A, Wahlroos S, Brancatisano R. Improvement in comorbid illness after placement of the Swedish Adjustable Gastric Band. Surg Obes Relat Dis 2008; 4: S39-S46

[508] Peluso L, Vanek VW. Efficacy of gastric bypass in the treatment of obesity-related comorbidities. Nutr Clin Pract 2007; 22: 22 - 28

[509] Gibson PG, Simpson JL. The overlap syndrome of asthma and COPD: what are its features and how important is it? Thorax 2009; 64: $728-735$

[510] Hardin M, Silverman EK, Barr RG et al. The clinical features of the overlap between COPD and asthma. Respir Res 2011; 12: 127

[511] Andersén H, Lampela P, Nevanlinna A et al. High hospital burden in overlap syndrome of asthma and COPD. Clin Respir J 2013; 7: 342 346

[512] Blanchette CM, Broder M, Ory C et al. Cost and utilization of COPD and asthma among insured adults in the US. Curr Med Res Opin 2009; 25: $1385-1392$

[513] de Marco R, Pesce G, Marcon A et al. The coexistence of asthma and chronic obstructive pulmonary disease (COPD): prevalence and risk factors in young, middle-aged and elderly people from the general population. PloS one 2013; 8: e62985

[514] Hardin M, Cho M, McDonald ML et al. The clinical and genetic features of COPD-asthma overlap syndrome. Eur Respir ] 2014; 44: 341 350

[515] Iwamoto H, Gao J, Koskela J et al. Differences in plasma and sputum biomarkers between COPD and COPD-asthma overlap. Eur Respir J 2014; 43: $421-429$

[516] Izquierdo-Alonso JL, Rodriguez-Gonzálezmoro JM, de Lucas-Ramos P et al. Prevalence and characteristics of three clinical phenotypes of chronic obstructive pulmonary disease (COPD). Respir Med 2013; 107: $724-731$

[517] Kauppi P, Kupiainen $\mathrm{H}$, Lindqvist A et al. Overlap syndrome of asthma and COPD predicts low quality of life. J Asthma 2011; 48: 279-285

[518] Kitaguchi Y, Komatsu Y, Fujimoto K et al. Sputum eosinophilia can predict responsiveness to inhaled corticosteroid treatment in patients with overlap syndrome of COPD and asthma. Int J Chron Obstruct Pulmon Dis 2012; 7: 283-289

[519] Marsh SE, Travers J, Weatherall M et al. Proportional classifications of COPD phenotypes. Thorax 2008; 63: 761 - 767

[520] Menezes AM, Montes de Oca M, Pérez-Padilla R et al. Increased risk of exacerbation and hospitalization in subjects with an overlap phenotype: COPD-asthma. Chest 2014; 145: 297-304

[521] Miravitlles M, Soler-Cataluña JJ, Calle M et al. Treatment of COPD by clinical phenotypes: putting old evidence into clinical practice. Eur Respir J 2013; 41: 1252 - 1256

[522] Pleasants RA, Ohar JA, Croft JB et al. Chronic obstructive pulmonary disease and asthma-patient characteristics and health impairment. COPD 2014; 11: $256-266$

[523] Putcha N, Drummond MB, Wise RA et al. Comorbidities and Chronic Obstructive Pulmonary Disease: Prevalence, Influence on Outcomes, and Management. Semin Respir Crit Care Med 2015; 36: $575-591$

[524] Rhee CK, Yoon HK, Yoo KH et al. Medical utilization and cost in patients with overlap syndrome of chronic obstructive pulmonary disease and asthma. COPD 2014; 11: $163-170$

[525] Shaya FT, Dongyi D, Akazawa MO et al. Burden of concomitant asthma and COPD in a Medicaid population. Chest 2008; 134: 14 19

[526] Tálamo C, de Oca MM, Halbert R et al. Diagnostic labeling of COPD in five Latin American cities. Chest 2007: 131:60-67
[527] Wurst KE, Shukla A, Muellerova $\mathrm{H}$ et al. Respiratory pharmacotherapy use in patients newly diagnosed with chronic obstructive pulmonary disease in a primary care setting in the UK: a retrospective cohort study. COPD 2014; 11: $521-530$

[528] Diaz-Guzman E, Khosravi M, Mannino DM. Asthma, chronic obstructive pulmonary disease, and mortality in the U.S. population. COPD 2011; 8: $400-407$

[529] Brightling CE, McKenna S, Hargadon B et al. Sputum eosinophilia and the short term response to inhaled mometasone in chronic obstructive pulmonary disease. Thorax 2005; 60: 193 - 198

[530] Leigh R, Pizzichini MM, Morris MM et al. Stable COPD: predicting benefit from high-dose inhaled corticosteroid treatment. Eur Respir J 2006; 27: $964-971$

[531] O'Donnell DE, Aaron S, Bourbeau J et al. Canadian Thoracic Society recommendations for management of chronic obstructive pulmonary disease - 2007 update. Can Respir J 2007; 14: 5B - 32B

[532] Barrecheguren M, Esquinas C, Miravitlles M. The asthma-chronic obstructive pulmonary disease overlap syndrome (ACOS): opportunities and challenges. Curr Opin Pulm Med 2015; 21: 74 - 79

[533] Brusselle GG, Bracke K, Lahousse L. Targeted therapy with inhaled corticosteroids in COPD according to blood eosinophil counts. Lancet Respir Med 2015; 3: 416-417

[534] Iqbal A, Barnes NC, Brooks ]. Is Blood Eosinophil Count a Predictor of Response to Bronchodilators in Chronic Obstructive Pulmonary Disease? Results from Post Hoc Subgroup Analyses Clin Drug Investig 2015; 35: 685-688

[535] Park HY, Lee H, Koh WJ et al. Association of blood eosinophils and plasma periostin with FEV1 response after 3-month inhaled corticosteroid and long-acting beta2-agonist treatment in stable COPD patients. Int J Chron Obstruct Pulmon Dis 2016; 11: 23 - 30

[536] Pascoe S, Locantore N, Dransfield MT et al. Blood eosinophil counts, exacerbations, and response to the addition of inhaled fluticasone furoate to vilanterol in patients with chronic obstructive pulmonary disease: a secondary analysis of data from two parallel randomised controlled trials. Lancet Respir Med 2015; 3: 435-442

[537] Pavord ID, Lettis S, Locantore $N$ et al. Blood eosinophils and inhaled corticosteroid/long-acting $\beta$ - 2 agonist efficacy in COPD. Thorax 2016; 71: $118-125$

[538] Siddiqui SH, Guasconi A, Vestbo ] et al. Blood Eosinophils: A Biomarker of Response to Extrafine Beclomethasone/Formoterol in Chronic Obstructive Pulmonary Disease. Am J Respir Crit Care Med 2015; 192: $523-525$

[539] Marple BF. Allergic rhinitis and inflammatory airway disease: interactions within the unified airspace. Am J Rhinol Allergy 2010; 24: $249-254$

[540] Guerra S, Sherrill DL, Martinez FD et al. Rhinitis as an independent risk factor for adult-onset asthma. J Allergy Clin Immunol 2002; 109: 419-425

[541] Bousquet J, Van Cauwenberge P, Khaltaev N. Allergic rhinitis and its impact on asthma. J Allergy Clin Immunol 2001; 108: S147-334

[542] Stevenson DD, Mathison DA, Tan EM et al. Provoking factors in bronchial asthma. Arch Intern Med 1975; 135: 777-783

[543] Settipane RJ, Hagy GW, Settipane GA. Long-term risk factors for developing asthma and allergic rhinitis: a 23-year follow-up study of college students. Allergy Proc 1994; 15: $21-25$

[544] Huovinen E, Kaprio ], Laitinen LA et al. Incidence and prevalence of asthma among adult Finnish men and women of the Finnish Twin Cohort from 1975 to 1990 , and their relation to hay fever and chronic bronchitis. Chest 1999; 115: 928 - 936

[545] Braman SS, Barrows AA, DeCotiis BA et al. Airway hyperresponsiveness in allergic rhinitis. A risk factor for asthma. Chest 1987; 91: $671-674$ 
[546] Ciprandi G, Cirillo I, Tosca MA et al. Bronchial hyperreactivity and spirometric impairment in patients with perennial allergic rhinitis. Int Arch Allergy Immunol 2004; 133: 14- 18

[547] Ciprandi G, Cirillo I, Tosca MA et al. Bronchial hyperreactivity and spirometric impairment in patients with seasonal allergic rhinitis. Respir Med 2004; 98: 826-831

[548] Ciprandi G, Cirillo I, Pistorio A et al. Impact of allergic rhinitis on asthma: effects on bronchodilation testing. Ann Allergy Asthma Immunol 2008; 101: 42-46

[549] Ciprandi G, Capasso M, Tosca MA. Early bronchial involvement in children with allergic rhinitis. Am J Rhinol Allergy 2011; 25: e30 e33

[550] Ciprandi G, Signori A, Cirillo I. Relationship between bronchial hyperreactivity and bronchodilation in patients with allergic rhinitis. Ann Allergy Asthma Immunol 2011; 106: 460 -466

[551] Braunstahl G], Overbeek SE, Kleinjan A et al. Nasal allergen provocation induces adhesion molecule expression and tissue eosinophilia in upper and lower airways. J Allergy Clin Immunol 2001; 107: 469 476

[552] Braunstahl G], Kleinjan A, Overbeek SE et al. Segmental bronchial provocation induces nasal inflammation in allergic rhinitis patients. Am J Respir Crit Care Med 2000; 161: 2051-2057

[553] Gaga M, Lambrou P, Papageorgiou N et al. Eosinophils are a feature of upper and lower airway pathology in non-atopic asthma, irrespective of the presence of rhinitis. Clin Exp Allergy 2000; 30: 663 669

[554] Becky Kelly EA, Busse WW, Jarjour NN. A comparison of the airway response to segmental antigen bronchoprovocation in atopic asthma and allergic rhinitis. J Allergy Clin Immunol 2003; 111: 79- 86

[555] Kurt E, Aktas A, Gulbas Z et al. The effects of natural pollen exposure on inflammatory cytokines and their relationship with nonspecific bronchial hyperresponsiveness in seasonal allergic rhinitis. Allergy Asthma Proc 2010; 31: 126-131

[556] Pinart M, Benet M, Annesi-Maesano I et al. Comorbidity of eczema, rhinitis, and asthma in IgE-sensitised and non-IgE-sensitised children in MeDALL: a population-based cohort study. Lancet Respir Med 2014; 2: $131-140$

[557] Brozek JL, Bousquet J, Baena-Cagnani CE et al. Allergic Rhinitis and its Impact on Asthma (ARIA) guidelines: 2010 revision. J Allergy Clin Immunol 2010; 126: 466-476

[558] Welsh PW, Stricker WE, Chu CP et al. Efficacy of beclomethasone nasal solution, flunisolide, and cromolyn in relieving symptoms of ragweed allergy. Mayo Clin Proc 1987; 62: 125 - 134

[559] Henriksen JM, Wenzel A. Effect of an intranasally administered corticosteroid (budesonide) on nasal obstruction, mouth breathing, and asthma. Am Rev Respir Dis 1984; 130: 1014-1018

[560] Corren J, Adinoff AD, Buchmeier AD et al. Nasal beclomethasone prevents the seasonal increase in bronchial responsiveness in patients with allergic rhinitis and asthma. J Allergy Clin Immunol 1992; 90: $250-256$

[561] Aubier M, Levy J, Clerici C et al. Different effects of nasal and bronchial glucocorticosteroid administration on bronchial hyperresponsiveness in patients with allergic rhinitis. Am Rev Respir Dis 1992; 146: $122-126$

[562] Dahl R, Nielsen LP, Kips J et al. Intranasal and inhaled fluticasone propionate for pollen-induced rhinitis and asthma. Allergy 2005; 60 : $875-881$

[563] Yatera K, Yamasaki K, Noguchi S et al. Prevalence of sinusitis and efficacy of intranasal corticosteroid treatment on asthmatic symptoms in asthmatic patients with rhinosinusitis: a pilot study. Int Forum Allergy Rhinol 2016; 6: 398-406
[564] Grant JA, Nicodemus CF, Findlay SR et al. Cetirizine in patients with seasonal rhinitis and concomitant asthma: prospective, randomized, placebo-controlled trial. J Allergy Clin Immunol 1995; 95: 923-932

[565] Spector SL, Nicodemus CF, Corren J et al. Comparison of the bronchodilatory effects of cetirizine, albuterol, and both together versus placebo in patients with mild-to-moderate asthma. J Allergy Clin Immunol 1995; 96: 174 -181

[566] Aubier M, Neukirch C, Peiffer C et al. Effect of cetirizine on bronchia hyperresponsiveness in patients with seasonal allergic rhinitis and asthma. Allergy 2001; 56: 35-42

[567] Crystal-Peters J, Neslusan C, Crown WH et al. Treating allergic rhinitis in patients with comorbid asthma: the risk of asthma-related hospitalizations and emergency department visits. J Allergy Clin Immunol 2002; 109: 57-62

[568] Corren J, Manning BE, Thompson SF et al. Rhinitis therapy and the prevention of hospital care for asthma: a case-control study. J Allergy Clin Immunol 2004; 113: 415-419

[569] Chun TH, Weitzen SH, Fritz GK. The asthma/mental health nexus in a population-based sample of the United States. Chest 2008; 134: $1176-1182$

[570] Katon W, Lozano P, Russo ] et al. The prevalence of DSM-IV anxiety and depressive disorders in youth with asthma compared with controls. J Adolesc Health 2007; 41: 455-463

[571] Scott KM, Von Korff M, Ormel J et al. Mental disorders among adults with asthma: results from the World Mental Health Survey. Gen Hosp Psychiatry 2007; 29: $123-133$

[572] Katon W], Richardson L, Russo J et al. Quality of mental health care for youth with asthma and comorbid anxiety and depression. Med Care 2006; 44: $1064-1072$

[573] Di Marco F, Verga M, Santus P et al. Close correlation between anxiety, depression, and asthma control. Respir Med 2010; 104: 22 28

[574] Goodwin RD, Pagura J, Cox B et al. Asthma and mental disorders in Canada: impact on functional impairment and mental health service use. J Psychosom Res 2010; 68: 165-173

[575] Kozyrskyj AL, Mai XM, McGrath P et al. Continued exposure to maternal distress in early life is associated with an increased risk of childhood asthma. Am J Respir Crit Care Med 2008; 177: 142 - 147

[576] Richardson LP, Russo JE, Lozano P et al. The effect of comorbid anxiety and depressive disorders on health care utilization and costs among adolescents with asthma. Gen Hosp Psychiatry 2008; 30: $398-406$

[577] Strine TW, Mokdad AH, Balluz LS et al. Impact of depression and anxiety on quality of life, health behaviors, and asthma control among adults in the United States with asthma, 2006. J Asthma 2008; 45: $123-133$

[578] von Leupoldt A, Dahme B. Psychological aspects in the perception of dyspnea in obstructive pulmonary diseases. Respir Med 2007; 101: $411-422$

[579] von Leupoldt A, Sommer T, Kegat S et al. Down-regulation of insular cortex responses to dyspnea and pain in asthma. Am J Respir Crit Care Med 2009; 180: 232-238

[580] von Leupoldt A, Chan PY, Bradley MM et al. The impact of anxiety on the neural processing of respiratory sensations. Neurolmage 2011; 55: $247-252$

[581] Lecheler J. Gesellschaftlicher Kontext: Schule. In: Lingner H, Schwartz FW, Schultz K, Hrsg. Volkskrankheit Asthma/COPD. Heidelberg : Springer Medizin Verlag; 2007

[582] Cookson H, Granell R, Joinson C et al. Mothers' anxiety during pregnancy is associated with asthma in their children. J Allergy Clin Immunol 2009; 123: 847-853

[583] Boulet LP. Influence of comorbid conditions on asthma. Eur Respir ] 2009; 33: $897-906$ 
[584] Ahnert ], Löffler S, Müller ] et al. [Systematic literature review on interventions in rehabilitation for children and adolescents with asthma bronchiale]. Rehabilitation 2010; 49: 147-159

[585] Menz G, Kronenberger $\mathrm{H}$, Lecheler J et al. [Pulmonary rehabilitation in asthma bronchiale]. Pneumologie 2007; 61: 710-718

[586] Petermann F, Warschburger P. Asthma bronchiale. Göttingen: Hogrefe; 2000

[587] Zacharasiewicz A. Maternal smoking in pregnancy and its influence on childhood asthma. ERJ Open Res 2016; 2: 00042-2016

[588] Schäfer T, Bauer CP, Beyer K et al. S3-Guideline on allergy prevention: 2014 update: Guideline of the German Society for Allergology and Clinical Immunology (DGAKI) and the German Society for Pediatric and Adolescent Medicine (DGKJ). Allergo | Int 2014; 23: $186-199$

[589] Schäfer T, Borowski C, Diepgen TL et al. [Evidence-based and consented guideline on allergy prevention]. J Dtsch Dermatol Ges 2004; 2: $1030-1036$

[590] Wahn U, von Mutius E. Childhood risk factors for atopy and the importance of early intervention. J Allergy Clin Immunol 2001; 107: $567-574$

[591] Becker AB. Is primary prevention of asthma possible? Pediatr Pulmonol 2000; 30: 63-72

[592] Haahtela T, Holgate S, Pawankar R et al. The biodiversity hypothesis and allergic disease: world allergy organization position statement. World Allergy Organ J 2013; 6: 3

[593] Karvonen AM, Hyvärinen A, Gehring U et al. Exposure to microbial agents in house dust and wheezing, atopic dermatitis and atopic sensitization in early childhood: a birth cohort study in rural areas. Clin Exp Allergy 2012; 42: 1246-1256

[594] Peters M, Kauth M, Schwarze J et al. Inhalation of stable dust extract prevents allergen induced airway inflammation and hyperresponsiveness. Thorax 2006; 61: 134-139

[595] Braun-Fahrländer C, Riedler J, Herz U et al. Environmental exposure to endotoxin and its relation to asthma in school-age children. N Engl J Med 2002; 347: 869-877

[596] Riedler ], Braun-Fahrländer C, Eder W et al. Exposure to farming in early life and development of asthma and allergy: a cross-sectional survey. Lancet 2001; 358: 1129-1133

[597] Ege M], Frei R, Bieli C et al. Not all farming environments protect against the development of asthma and wheeze in children. J Allergy Clin Immunol 2007; 119: 1140-1147

[598] Chan-Yeung M, Becker A. Primary prevention of childhood asthma and allergic disorders. Curr Opin Allergy Clin Immunol 2006; 6: $146-151$

[599] Oddy WH, Holt PG, Sly PD et al. Association between breast feeding and asthma in 6 year old children: findings of a prospective birth cohort study. BMJ 1999; 319: 815-819

[600] Maas T, Kaper J, Sheikh A et al. Mono and multifaceted inhalant and/ or food allergen reduction interventions for preventing asthma in children at high risk of developing asthma. Cochrane Database Syst Rev 2009; 3: CD006480

[601] Hide DW, Matthews S, Tariq S et al. Allergen avoidance in infancy and allergy at 4 years of age. Allergy 1996; 51: 89-93

[602] Boyle RJ, lerodiakonou D, Khan T et al. Hydrolysed formula and risk of allergic or autoimmune disease: systematic review and meta-analysis. BMJ 2016; 352: i974

[603] von Berg A, Filipiak-Pittroff B, Schulz H et al. Allergic manifestation 15 years after early intervention with hydrolyzed formulas-the GINI Study. Allergy 2016; 71: 210-219

[604] von Berg A, Filipiak-Pittroff B, Krämer $U$ et al. Preventive effect of hydrolyzed infant formulas persists until age 6 years: long-term results from the German Infant Nutritional Intervention Study (GINI). J Allergy Clin Immunol 2008; 121: 1442-1447
[605] von Berg A, Krämer U, Link E et al. Impact of early feeding on childhood eczema: development after nutritional intervention compared with the natural course - the GINIplus study up to the age of 6 years. Clin Exp Allergy 2010; 40: 627-636

[606] Schoetzau A, Gehring U, Wichmann HE. Prospective cohort studies using hydrolysed formulas for allergy prevention in atopy-prone newborns: a systematic review. Eur J Pediatr 2001; 160: 323-332

[607] Vandenplas Y, Hauser B, Van den Borre $C$ et al. The long-term effect of a partial whey hydrolysate formula on the prophylaxis of atopic disease. Eur J Pediatr 1995; 154: 488 - 494

[608] Azad MB, Coneys JG, Kozyrskyj AL et al. Probiotic supplementation during pregnancy or infancy for the prevention of asthma and wheeze: systematic review and meta-analysis. BMJ 2013; 347: f6471

[609] Grüber C, van Stuijvenberg M, Mosca F et al. Reduced occurrence of early atopic dermatitis because of immunoactive prebiotics among low-atopy-risk infants. J Allergy Clin Immunol 2010; 126: 791 - 797

[610] Kuitunen M, Kukkonen K, Juntunen-Backman K et al. Probiotics prevent IgE-associated allergy until age 5 years in cesarean-delivered children but not in the total cohort. J Allergy Clin Immunol 2009; 123: $335-341$

[611] Kopp MV, Hennemuth I, Heinzmann A et al. Randomized, doubleblind, placebo-controlled trial of probiotics for primary prevention: no clinical effects of Lactobacillus GG supplementation. Pediatrics 2008; 121: e850-856

[612] Kukkonen K, Savilahti E, Haahtela T et al. Probiotics and prebiotic galacto-oligosaccharides in the prevention of allergic diseases: a randomized, double-blind, placebo-controlled trial. J Allergy Clin Immunol 2007; 119: 192 - 198

[613] Moro G, Arslanoglu S, Stahl B et al. A mixture of prebiotic oligosaccharides reduces the incidence of atopic dermatitis during the first six months of age. Arch Dis Child 2006; 91: 814-819

[614] Dunstan JA, Mori TA, Barden A et al. Fish oil supplementation in pregnancy modifies neonatal allergen-specific immune responses and clinical outcomes in infants at high risk of atopy: a randomized, controlled trial. J Allergy Clin Immunol 2003; 112: 1178-1184

[615] Rautava S, Kalliomäki M, Isolauri E. Probiotics during pregnancy and breast-feeding might confer immunomodulatory protection against atopic disease in the infant. J Allergy Clin Immunol 2002; 109: 119121

[616] Kalliomäki M, Salminen S, Arvilommi H et al. Probiotics in primary prevention of atopic disease: a randomised placebo-controlled trial. Lancet 2001; 357: 1076-1079

[617] Zutavern A, Brockow I, Schaaf B et al. Timing of solid food introduction in relation to eczema, asthma, allergic rhinitis, and food and inhalant sensitization at the age of 6 years: results from the prospective birth cohort study LISA. Pediatrics 2008; 121: e44- 52

[618] Filipiak B, Zutavern A, Koletzko $S$ et al. Solid food introduction in relation to eczema: results from a four-year prospective birth cohort study. J Pediatr 2007; 151: $352-358$

[619] Flexeder C, Thiering E, Brüske I et al. Growth velocity during infancy and onset of asthma in school-aged children. Allergy 2012; 67: $257-264$

[620] Zammit C, Liddicoat H, Moonsie I et al. Obesity and respiratory diseases. Int J Gen Med 2010; 3: 335 - 343

[621] Delgado J, Barranco P, Quirce S. Obesity and asthma. J Investig Allergol Clin Immunol 2008; 18: 420-425

[622] Beuther DA, Sutherland ER. Overweight, obesity, and incident asthma: a meta-analysis of prospective epidemiologic studies. Am J Respir Crit Care Med 2007; 175: 661 - 666

[623] Ford ES. The epidemiology of obesity and asthma. J Allergy Clin Immunol 2005; 115: 897-909 
[624] Wongtrakool C, Wang N, Hyde DM et al. Prenatal nicotine exposure alters lung function and airway geometry through $\alpha 7$ nicotinic receptors. Am J Respir Cell Mol Biol 2012; 46: 695-702

[625] Burke H, Leonardi-Bee J, Hashim A et al. Prenatal and passive smoke exposure and incidence of asthma and wheeze: systematic review and meta-analysis. Pediatrics 2012; 129: 735 - 744

[626] Horak F, Fazekas T, Zacharasiewicz A et al. [The Fetal Tobacco Syndrome - A statement of the Austrian Societies for General- and Family Medicine (ÖGAM), Gynecology and Obstetrics (ÖGGG), Hygiene, Microbiology and Preventive Medicine (ÖGHMP), Pediatrics and Adolescence Medicine (ÖGKJ) as well as Pneumology (ÖGP)]. Wien Klin Wochenschr 2012; 124: 129-145

[627] Gilliland FD, Li YF, Peters JM. Effects of maternal smoking during pregnancy and environmental tobacco smoke on asthma and wheezing in children. Am J Respir Crit Care Med 2001; 163: 429-436

[628] Tariq SM, Hakim EA, Matthews SM et al. Influence of smoking on asthmatic symptoms and allergen sensitisation in early childhood. Postgrad Med J 2000; 76: 694-699

[629] Strachan DP, Cook DG. Health effects of passive smoking. 6. Parental smoking and childhood asthma: longitudinal and case-control studies. Thorax 1998; 53: 204-212

[630] Tarlo SM, Liss GM, Yeung KS. Changes in rates and severity of compensation claims for asthma due to diisocyanates: a possible effect of medical surveillance measures. Occup Environ Med 2002; 59: $58-62$

[631] Merget R, Caspari C, Dierkes-Globisch A et al. Effectiveness of a medical surveillance program for the prevention of occupational asthma caused by platinum salts: a nested case-control study. J Allergy Clin Immunol 2001; 107: 707-712

[632] Schönberger H], Maas T, Dompeling E et al. Compliance of asthmatic families with a primary prevention programme of asthma and effectiveness of measures to reduce inhalant allergens-a randomized trial. Clin Exp Allergy 2004; 34: 1024- 1031

[633] Lodge C], Lowe AJ, Gurrin LC et al. House dust mite sensitization in toddlers predicts current wheeze at age 12 years. J Allergy Clin Immunol 2011; 128: 782 - 788

[634] Celedón JC, Milton DK, Ramsey CD et al. Exposure to dust mite allergen and endotoxin in early life and asthma and atopy in childhood. J Allergy Clin Immunol 2007; 120: 144 - 149

[635] Schram-Bijkerk D, Doekes G, Boeve M et al. Nonlinear relations between house dust mite allergen levels and mite sensitization in farm and nonfarm children. Allergy 2006; 61: 640-647

[636] Gøtzsche PC, Johansen HK, Schmidt LM et al. House dust mite control measures for asthma. Cochrane Database Syst Rev 2004; 4: CD001187

[637] Lau S, Illi S, Sommerfeld C et al. Early exposure to house-dust mite and cat allergens and development of childhood asthma: a cohort study. Multicentre Allergy Study Group. Lancet 2000; 356: 1392 1397

[638] Gøtzsche PC, Hammarquist C, Burr M. House dust mite control measures in the management of asthma: meta-analysis. BMJ 1998; 317: $1105-1110$

[639] Kuehr ], Frischer T, Meinert R et al. Mite allergen exposure is a risk for the incidence of specific sensitization. J Allergy Clin Immunol 1994; 94: $44-52$

[640] Sporik R, Holgate ST, Platts-Mills TA et al. Exposure to house-dust mite allergen (Der p I) and the development of asthma in childhood. A prospective study. N Engl J Med 1990; 323: $502-507$

[641] Lau S, Falkenhorst G, Weber A et al. High mite-allergen exposure increases the risk of sensitization in atopic children and young adults. J Allergy Clin Immunol 1989; 84: 718-725
[642] Perzanowski MS, Chew GL, Divjan A et al. Cat ownership is a risk factor for the development of anti-cat IgE but not current wheeze at age 5 years in an inner-city cohort. J Allergy Clin Immunol 2008; 121 : $1047-1052$

[643] Takkouche B, González-Barcala F], Etminan M et al. Exposure to furry pets and the risk of asthma and allergic rhinitis: a meta-analysis. Allergy 2008; 63: 857-864

[644] Bufford JD, Gern JE. Early exposure to pets: good or bad? Curr Allergy Asthma Rep 2007; 7: 375-382

[645] Waser M, von Mutius E, Riedler J et al. Exposure to pets, and the association with hay fever, asthma, and atopic sensitization in rural children. Allergy 2005; 60: $177-184$

[646] Brussee JE, Smit HA, van Strien RT et al. Allergen exposure in infancy and the development of sensitization, wheeze, and asthma at 4 years. J Allergy Clin Immunol 2005; 115: 946-952

[647] Svanes C, Heinrich J, Jarvis D et al. Pet-keeping in childhood and adult asthma and hay fever: European community respiratory health survey. J Allergy Clin Immunol 2003; 112: 289-300

[648] Ownby DR, Johnson CC, Peterson EL. Exposure to dogs and cats in the first year of life and risk of allergic sensitization at 6 to 7 years of age. JAMA 2002; 288: $963-972$

[649] Custovic A, Simpson BM, Simpson A et al. Effect of environmental manipulation in pregnancy and early life on respiratory symptoms and atopy during first year of life: a randomised trial. Lancet 2001; 358: $188-193$

[650] Melén E, Wickman M, Nordvall SL et al. Influence of early and current environmental exposure factors on sensitization and outcome of asthma in pre-school children. Allergy 2001; 56: 646-652

[651] Remes ST, Castro-Rodriguez JA, Holberg C] et al. Dog exposure in infancy decreases the subsequent risk of frequent wheeze but not of atopy. J Allergy Clin Immunol 2001; 108: 509-515

[652] Hesselmar B, Aberg N, Aberg B et al. Does early exposure to cat or dog protect against later allergy development? Clin Exp Allergy 1999; 29: $611-617$

[653] Perzanowski MS, Rönmark E, Platts-Mills TA et al. Effect of cat and dog ownership on sensitization and development of asthma among preteenage children. Am J Respir Crit Care Med 2002; 166: 696-702

[654] Campo P, Kalra HK, Levin L et al. Influence of dog ownership and high endotoxin on wheezing and atopy during infancy. J Allergy Clin Immunol 2006; 118: 1271 - 1278

[655] Litonjua AA, Milton DK, Celedon JC et al. A longitudinal analysis of wheezing in young children: the independent effects of early life exposure to house dust endotoxin, allergens, and pets. J Allergy Clin Immunol 2002; 110: 736 - 742

[656] Chen CM, Morgenstern V, Bischof W et al. Dog ownership and contact during childhood and later allergy development. Eur Respir J 2008; 31: $963-973$

[657] Tischer CG, Hohmann C, Thiering E et al. Meta-analysis of mould and dampness exposure on asthma and allergy in eight European birth cohorts: an ENRIECO initiative. Allergy 2011; 66: 1570 - 1579

[658] Tischer C, Chen CM, Heinrich J. Association between domestic mould and mould components, and asthma and allergy in children: a systematic review. Eur Respir J 2011; 38: 812-824

[659] Tischer C, Gehring U, Chen CM et al. Respiratory health in children, and indoor exposure to (1,3)- $\beta$-D-glucan, EPS mould components and endotoxin. Eur Respir J 2011; 37: 1050 - 1059

[660] Fisk W], Lei-Gomez Q, Mendell MJ. Meta-analyses of the associations of respiratory health effects with dampness and mold in homes. Indoor air 2007; 17: $284-296$

[661] Wiesmüller GA, Heinzow B, Aurbach U et al. [Medical Diagnostics for Mold Exposure Indoors]. Pneumologie 2016; 70: 699-741

[662] Gasana J, Dillikar D, Mendy A et al. Motor vehicle air pollution and asthma in children: a meta-analysis. Environ Res 2012; 117: 36-45 
[663] Sonnenschein-van der Voort AM, de Kluizenaar Y, Jaddoe VW et al. Air pollution, fetal and infant tobacco smoke exposure, and wheezing in preschool children: a population-based prospective birth cohort. Environ Health 2012; 11: 91

[664] Morgenstern V, Zutavern A, Cyrys ] et al. Atopic diseases, allergic sensitization, and exposure to traffic-related air pollution in children. Am J Respir Crit Care Med 2008; 177: 1331 - 1337

[665] Morgenstern V, Zutavern A, Cyrys J et al. Respiratory health and individual estimated exposure to traffic-related air pollutants in a cohort of young children. Occup Environ Med 2007; 64: 8-16

[666] Nicolai T, Carr D, Weiland SK et al. Urban traffic and pollutant exposure related to respiratory outcomes and atopy in a large sample of children. Eur Respir J 2003; 21: 956 - 963

[667] Scott M, Roberts G, Kurukulaaratchy RJ et al. Multifaceted allergen avoidance during infancy reduces asthma during childhood with the effect persisting until age 18 years. Thorax 2012; 67: 1046-1051

[668] van Schayck OC, Maas T, Kaper J et al. Is there any role for allergen avoidance in the primary prevention of childhood asthma? J Allergy Clin Immunol 2007; 119: 1323-1328

[669] Chan-Yeung M, Ferguson A, Watson W et al. The Canadian Childhood Asthma Primary Prevention Study: outcomes at 7 years of age. J Allergy Clin Immunol 2005; 116: 49-55

[670] Schönberger H], Dompeling E, Knottnerus JA et al. The PREVASC study: the clinical effect of a multifaceted educational intervention to prevent childhood asthma. Eur Respir J 2005; 25: 660-670

[671] Becker A, Watson W, Ferguson A et al. The Canadian asthma primary prevention study: outcomes at 2 years of age. J Allergy Clin Immunol 2004; 113: $650-656$

[672] Arshad SH, Bateman B, Matthews SM. Primary prevention of asthma and atopy during childhood by allergen avoidance in infancy: a randomised controlled study. Thorax 2003; 58: 489-493

[673] Marra F, Marra CA, Richardson K et al. Antibiotic use in children is associated with increased risk of asthma. Pediatrics 2009; 123 : $1003-1010$

[674] Stensballe LG, Simonsen J, Jensen SM et al. Use of antibiotics during pregnancy increases the risk of asthma in early childhood. J Pediatr 2013; 162: $832-838$

[675] Celedón JC, Fuhlbrigge A, Rifas-Shiman S et al. Antibiotic use in the first year of life and asthma in early childhood. Clin Exp Allergy 2004; 34: $1011-1016$

[676] Etminan M, Sadatsafavi M, Jafari S et al. Acetaminophen use and the risk of asthma in children and adults: a systematic review and metaanalysis. Chest 2009; 136: $1316-1323$

[677] Eyers S, Weatherall M, Jefferies S et al. Paracetamol in pregnancy and the risk of wheezing in offspring: a systematic review and metaanalysis. Clin Exp Allergy 2011; 41: 482-489

[678] Thavagnanam S, Fleming J, Bromley A et al. A meta-analysis of the association between Caesarean section and childhood asthma. Clin Exp Allergy 2008; 38: 629-633

[679] Azad MB, Konya T, Maughan H et al. Gut microbiota of healthy Canadian infants: profiles by mode of delivery and infant diet at 4 months. CMAJ 2013; 185: 385-394

[680] Alm JS, Lilja G, Pershagen G et al. BCG vaccination does not seem to prevent atopy in children with atopic heredity. Allergy 1998; 53: 537

[681] Alm JS, Lilja G, Pershagen G et al. Early BCG vaccination and development of atopy. Lancet 1997; 350: 400-403

[682] Blanc PD, Toren K. How much adult asthma can be attributed to occupational factors? Am J Med 1999; 107: 580 - 587

[683] Balmes ], Becklake M, Blanc P et al. American Thoracic Society Statement: Occupational contribution to the burden of airway disease. Am J Respir Crit Care Med 2003; 167: 787 - 797
[684] Mapp CE, Boschetto P, Maestrelli P et al. Occupational asthma. Am J Respir Crit Care Med 2005; 172: 280-305

[685] Kogevinas M, Zock JP, Jarvis D et al. Exposure to substances in the workplace and new-onset asthma: an international prospective population-based study (ECRHS-II). Lancet 2007; 370: 336-341

[686] Baur X, Bakehe P, Vellguth H. Bronchial asthma and COPD due to irritants in the workplace - an evidence-based approach. J Occup Med Toxicol 2012; 7: 19

[687] Burge PS, Moore VC, Robertson AS. Sensitization and irritant-induced occupational asthma with latency are clinically indistinguishable. Occup Med 2012; 62: 129-133

[688] Brooks SM, Weiss MA, Bernstein IL. Reactive airways dysfunction syndrome (RADS). Persistent asthma syndrome after high level irritant exposures. Chest 1985; 88: $376-384$

[689] Nowak D. Chemosensory irritation and the lung. Int Arch Occup Environ Health 2002; 75: 326-331

[690] Le Moual N, Siroux V, Pin I et al. Asthma severity and exposure to occupational asthmogens. Am J Respir Crit Care Med 2005; 172: $440-445$

[691] Baur X. A compendium of causative agents of occupational asthma. J Occup Med Toxicol 2013; 8: 15

[692] Wilken D, Baur X, Barbinova L et al. What are the benefits of medical screening and surveillance? Eur Respir Rev 2012; 21: 105-111

[693] Vandenplas O, Ghezzo H, Munoz X et al. What are the questionnaire items most useful in identifying subjects with occupational asthma? Eur Respir ] 2005; 26: 1056-1063

[694] Baur X, Drexler H, Kraus T et al. Arbeitsmedizinische Leitlinie der Deutschen Gesellschaft für Arbeitsmedizin und Umweltmedizin e.V. (DGAUM). Arbeitsplatzbezogener Inhalationstest (AIT). Arbeitsmed Sozialmed Umweltmed 2010; 45: 434-441

[695] http://www.dguv.de/formtexte/aerzte/index.jsp

[696] Bundesregierung. Berufskrankheiten-Verordnung vom 31. Oktober 1997 (BGBI. I S. 2623), die zuletzt durch Artikel 1 der Verordnung vom 11. Juni 2009 (BGBI. I S. 1273) geändert worden ist. BGBI I; 2009

[697] Vandenplas O, Dressel H, Nowak D et al. What is the optimal management option for occupational asthma? Eur Respir Rev 2012; 21: 97- 104

[698] Heederik D, Henneberger PK, Redlich CA. Primary prevention: exposure reduction, skin exposure and respiratory protection. Eur Respir Rev 2012; 21: 112 - 124

[699] Baur X, Sigsgaard T, Aasen TB et al. Guidelines for the management of work-related asthma. Eur Respir ] 2012; 39: 529-545

[700] Nicholson PJ, Cullinan P, Taylor AJ et al. Evidence based guidelines for the prevention, identification, and management of occupational asthma. Occup Environ Med 2005; 62: 290 - 299

[701] Schatz M, Harden K, Forsythe A et al. The course of asthma during pregnancy, post partum, and with successive pregnancies: a prospective analysis. J Allergy Clin Immunol 1988; 81: 509-517

[702] Gluck JC, Gluck PA. The effect of pregnancy on the course of asthma. Immunol Allergy Clin North Am 2006; 26: 63 - 80

[703] Murphy VE, Gibson PG, Smith R et al. Asthma during pregnancy: mechanisms and treatment implications. Eur Respir ] 2005; 25 : $731-750$

[704] Murphy VE, Clifton VL, Gibson PG. Asthma exacerbations during pregnancy: incidence and association with adverse pregnancy outcomes. Thorax 2006; 61: 169-176

[705] Cydulka RK, Emerman CL, Schreiber D et al. Acute asthma among pregnant women presenting to the emergency department. Am J Respir Crit Care Med 1999; 160: 887-892 
[706] Murphy VE, Powell H, Wark PA et al. A prospective study of respiratory viral infection in pregnant women with and without asthma. Chest 2013; 144: $420-427$

[707] Mendola P, Laughon SK, Männistö TI et al. Obstetric complications among US women with asthma. Am J Obstet Gynecol 2013; 208: $127-128 . e 1$

[708] Demissie K, Breckenridge MB, Rhoads GG. Infant and maternal outcomes in the pregnancies of asthmatic women. Am J Respir Crit Care Med 1998; 158: 1091 - 1095

[709] Murphy VE, Gibson PG. Asthma in pregnancy. Clin Chest Med 2011; 32: $93-110$

[710] Salihu HM, Wilson RE. Epidemiology of prenatal smoking and perinatal outcomes. Early Hum Dev 2007; 83: 713 - 720

[711] Hodyl NA, Stark MJ, Scheil W et al. Perinatal outcomes following maternal asthma and cigarette smoking during pregnancy. Eur Respir J 2014; 43: $704-716$

[712] Lumley J, Oliver SS, Chamberlain C et al. Interventions for promoting smoking cessation during pregnancy. Cochrane Database Syst Rev 2004; 4: CD001055

[713] Juniper EF, Bousquet J, Abetz L et al. Identifying 'well-controlled' and 'not well-controlled' asthma using the Asthma Control Questionnaire. Respir Med 2006; 100: 616-621

[714] Lim AS, Stewart K, Abramson M] et al. Multidisciplinary Approach to Management of Maternal Asthma (MAMMA): a randomized controlled trial. Chest 2014; 145: 1046 - 1054
[715] National Heart, Lung, and Blood Institute; National Asthma Education and Prevention Program Asthma and Pregnancy Working Group. NAEPP expert panel report. Managing asthma during pregnancy: recommendations for pharmacologic treatment-2004 update. J Allergy Clin Immunol 2005; 115: 34-46

[716] Lim A, Stewart K, König K et al. Systematic review of the safety of regular preventive asthma medications during pregnancy. Ann Pharmacother 2011; 45: 931 - 945

[717] Vogt H, Lindström K, Bråbäck L et al. Preterm birth and inhaled corticosteroid use in 6- to 19-year-olds: a Swedish national cohort study. Pediatrics 2011; 127: $1052-1059$

[718] Wendel PJ, Ramin SM, Barnett-Hamm C et al. Asthma treatment in pregnancy: a randomized controlled study. Am J Obstet Gynecol 1996; 175: $150-154$

[719] Schatz M, Leibman C. Inhaled corticosteroid use and outcomes in pregnancy. Ann Allergy Asthma Immunol 2005; 95: $234-238$

[720] Beschluss des Gemeinsamen Bundesausschusses über eine Änderung der Arzneimittel-Richtlinie (AM- RL) in Anlage IV: Therapiehinweis zu Omalizumab. Dtsch Arztebl 2011; 108: A600/ B-488/C-488

[721] Mylonas I. Antibiotic chemotherapy during pregnancy and lactation period: aspects for consideration. Arch Gynecol Obstet 2011; 283: $7-18$

[722] Nelson-Piercy C. Asthma in pregnancy. Thorax 2001; 56: 325-328

Hinweis: Dieser Beitrag wurde gemäß Errata vom 15.1.2018 und 7.11.2018 geändert. 